LBNL-45254

CBP Note-331

\title{
Studies of Beam Dynamics in Relativistic Klystron Two-Beam Accelerators
}

\author{
Steven M. Lidia \\ Ph.D. Thesis \\ Department of Physics \\ University of California, Davis \\ and \\ Center for Beam Physics \\ Accelerator and Fusion Research Division \\ E.O. Lawrence Berkeley National Laboratory
}

December 1999

This work was supported by the U.S. Department of Energy under Contract No. AC03-76SF00098. 



\title{
Studies Of Beam Dynamics In Relativistic Klystron Two-Beam Accelerators
}

\author{
By \\ Steven Michael Lidia \\ B.S., University of California, Berkeley, 1991 \\ DISSERTATION
}

Submitted in partial satisfaction of the requirements for the degree of

DOCTOR OF PHILOSOPHY

in

Physics

in the

OFFICE OF GRADUATE STUDIES

of the

UNIVERSITY OF CALIFORNIA

DAVIS

Approved:

\author{
Prof. Winston Ko (Chair) \\ Dr. Simon $\mathrm{Yu}$ \\ Dr. Andrew Sessler \\ Committee in Charge
}

1999 
Studies of Beam Dynamics In Relativistic Klystron Two-Beam Accelerators ${ }^{1}$

\begin{abstract}
$\underline{\text { Abstract }}$
Two-beam accelerators (TBAs) based upon free-electron lasers (FELs) or relativistic klystrons (RK-TBAs) have been proposed as efficient power sources for next generation high-energy linear colliders. Studies have demonstrated the possibility of building TBAs from X-band ( $\sim 8-12 \mathrm{GHz})$ through Ka-band $(\sim 30-35 \mathrm{GHz})$ frequency regions. Provided that further prototyping shows stable beam propagation with minimal current loss and production of good quality, high-power rf fields, this technology is compatible with current schemes for electron-positron colliders in the multi- $\mathrm{TeV}$ center-of-mass scale.

A new method of simulating the beam dynamics in accelerators of this type has been developed in this dissertation. There are three main components to this simulation. The first is a tracking algorithm to generate nonlinear transfer maps for pushing noninteracting particles through the external fields. A mapping algorithm is used so that tens or hundreds of thousands of macroparticles can be pushed from the solution of a few hundreds of differential equations. This is a great cost-savings device from the standpoint of CPU cycles. It can increase by several orders of magnitude the number of macroparticles that take place in the simulation, enabling more accurate modeling of the evolution of the beam distribution and enhanced sensitivity to effects due to the beam's halo. The second component is a 3D Particle-In-Cell (PIC) algorithm that solves a set of Helmholtz equations for the self-fields, including the conducting boundary condition, and generates impulses that are interleaved with the nonlinear maps by means of a split-operator algorithm. The Helmholtz equations are solved by a multi-grid algorithm. The third component is an equivalent circuit equation solver that advances the modal rf cavity fields in time due to excitation by the modulated beam. The beam-cavity interaction is analyzed and divided naturally into two distinct times scales. The fast-time scale contains details of individual particle trajectories, and the work done upon the cavity mode by the particles and vice-versa. A bunch-averaged shunt impedance, which contains information related to beam loading effects, is derived and calculated. This provides the bridge between the fast and slow time scales. Once averaged over the fast time scale, the ordinary differential circuit equation can be integrated numerically, or solved analytically in the case of short (slow-) time scale intervals. Phase-space partitioning is realized by a multi-fiducial algorithm which can produce high accuracy with lower-order dynamical calculations. This additional technique is used in all three main
\end{abstract}

\footnotetext{
${ }^{1}$ This work was supported by the U.S. Department of Energy under Contract No. AC03-76SF00098.
} 
sectors of the calculation: the single particle mapping. the self-field calculator, and the circuit model solver. It provides a way to guarantee self-consistency.

The RTA project is described, and the simulation code is used to design the latter portions of the experiment. Detailed calculations of the beam dynamics and of the rf cavity output are presented and discussed. A beamline design is presented that will generate nearly $1.2 \mathrm{GW}$ of power from 40 input, gain, and output rf cavities over a 10 $\mathrm{m}$ distance. The simulations show that beam current losses are acceptable, and that longitudinal and transverse focusing techniques are sufficiently capable of maintaining a high degree of beam quality along the entire beamline.

Additional experimental efforts are described. The first is the commissioning of the RTA injector. This electron gun produces a $1 \mathrm{MV}, 600 \mathrm{~A}$ beam over a $250 \mathrm{~ns}$ pulse length. The post-injector beamline is described, and the battery of diagnostics is presented, with initial results reported. The second effort concerns the recent tests of beam bunching, transport, and rf power extraction from a cavity at the CEA/CESTA facility. This experiment seeks to extend the RK-TBA concept from the X-band to Ka-band frequencies. The PIVAIR injector and beamline are presented, as are the freeelectron laser, the transport region and the power extraction cavitites. Results of the bunching and rf power measurements are presented and discussed. Comparisons are made with the results of simulations to establish benchmarking of our codes. 


\section{Acknowledgments}

There are many people whom I would like to heartily thank for their assistance.

First of all, I must express my immense gratitude to Simon Yu for taking me on as a graduate student. The last several years of working with him has been an educational experience unparalled. He has provided generous guidance and support, much more than a graduate student could ever expect. I thank Winston Ko for providing the academic and 'philosophical' support to a graduate student who wanted to perform his research in a field outside that of his faculty advisor.

I wish to express my gratitude toward the entire RTA project team, with whom I have worked closely over the last few years: Andy Sessler, Glen Westenskow, Tim Houck, Enrique Henestroza, Shmuel Eylon, Dave Vanecek, and Wayne Greenway. They have all contributed to my maturing as a physicist and a researcher, and their presence is felt throughout this entire dissertation. Glen and Tim, especially, have provided numerous resources, expertise, and patience during my training as an experimentalist.

I thank Rob Ryne for the initial instruction and direction in working with these kinds of beam transport simulations. Much of this work was begun during a visit to Los Alamos in early 1997, and I want to thank the entire LANSCE-1 group for their hospitality during my stay. Bill Fawley and Etienne Forest have each aided me in small and large ways during my construction of this dissertation.

From the Center for Beam Physics at LBL, I wish to thank Swapan Chattopadhyay, Joy Kono, and Olivia Wong for their support of our program. Numerous people in the HIF group at LBL and at LLNL deserve special note. I gratefully acknowledge the very informative conversations I've had with Ed Lee, Peter Seidl, Joe Kwan, John Barnard, Steve Lund, Christine Celata, Dave Grote, and Andy Faltens.

I have had long and fruitful collaborations and associations with several scientists at other institutions. I thank David Whittum and Roger Carr of SLAC for their continued professional and personal support. David graciously took me on as a student one summer and everything I know about free electron lasers and cavity circuit models I owe 
to him. Roger has provided much appreciated emotional and financial support during several long periods during when I was deciding what I wanted to do. Kwang-Je Kim deserves special mention for providing me with my first chance to perform some interesting research, and for inciting the desire to pursue more advanced graduate training.

I want to thank Jack Donohue and Yann Meurdesoif at the University of Bordeaux and Thibaut Lefevre, Jacques Gardelle, Jean-Luc Rullier, Christophe Vermare, and Daniel Gogny of the CESTA facility of the Commisariat a l'Énergie Atomique for the productive collaboration on Ka-band TBAs over the past three years. I would also like to thank them all for the wonderful hospitality (and cooking!) shown towards me during my stay there in early 1999. Thanks also to Walter Wuensch and Colin Johnson of CERN for their assistance in manufacturing the cavities we used in the experiments at CESTA. Many thanks go to Warner Bruns of TET-Berlin for making his GdFidL code available, and for answering my many questions and requests.

Of course, this work could not have completed with the love and support of my family. Thanks Mom, Dad, Lori, Dave, Jenny, Kaitlin, and Betty for being there and being patient with a sometimes harried son, brother, uncle, and grandson.

And, of course, there are the friends who have provided me with many a meal to chow upon, a shoulder to cry upon, a bed to lie upon, a drink to quaff, and company to share in our waltz through life. Thank you Matt and Bethany, Winter and Matt, Jeremy, Dave, Marc, Yola, Laura, Nanda, Mike and Jody, Sharad, Subham, Eric, Kaarin and Robert, Katherine and Mark, Christine, Ed, David and Doreen, Carey, Robert, Celeste and Naomi, and Wendy. You've all done me more of a service than you can possibly know.

And finally, my thanks to David Sutter and Mark Wilson for providing the funding to make this work possible in the first place. This work was performed under the auspices of the U.S. Department of Energy under contract AC03-76SF00098. 


\section{Contents}

$\begin{array}{lll}\text { I Background and Overview } & 1\end{array}$

1 Introduction $\quad 4$

2 Considerations of High Energy Linear Colliders $\quad 9$

2.1 Particle Physics at the $\mathrm{TeV}$ Scale . . . . . . . . . . . . . 9

2.2 Linear Collider Physics at the $\mathrm{TeV}$ Scale . . . . . . . . . . . 10

2.2 .1 Interaction Point . . . . . . . . . . . . . . . 10

2.2.2 High-Gradient Linac and RF Power Source . . . . . . . . . . . 13

3 Relativistic Klystron Two-Beam Accelerators 23

3.1 Power Source Requirements for an NLC Upgrade to $1.0 \mathrm{TeV}$. . . . . . . 23

3.2 TBNLC Architecture . . . . . . . . . . . . . . . . . 24

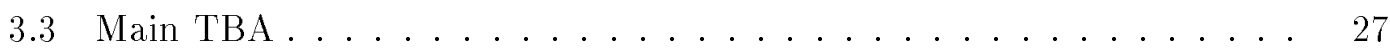

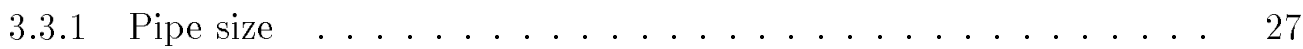

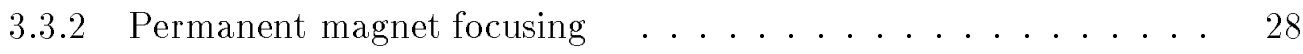

3.3.3 Steering and focusing corrections . . . . . . . . . . . 29

3.3 .4 Induction Cores . . . . . . . . . . . . . . . . 30

3.3.5 Pulsed Power . . . . . . . . . . . . . . . . 31

3.3.6 Acceleration Gap . . . . . . . . . . . . . . . 32

3.3 .7 RF Extraction cavities . . . . . . . . . . . . 33

3.4 Front End and Back End . . . . . . . . . . . . . . . . . 34

3.4 .1 Pre-chopper . . . . . . . . . . . . . . . 34

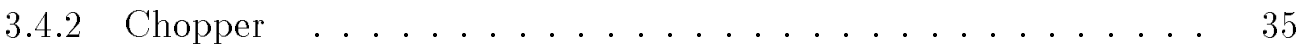

3.4 .3 Adiabatic Compressor . . . . . . . . . . . . . . 35

3.4 .4 Afterburner . . . . . . . . . . . . . . 36

3.4.5 Beam-dump ...................... 36 
II Methods of Simulation $\quad 37$

4 Introduction $\quad 40$

5 The Model Hamiltonian $\quad 45$

5.1 Introduction . . . . . . . . . . . . . . . . . 45

5.2 Derivation from the Action . . . . . . . . . . . . 46

5.2 .1 Poincaré-Cartan Form . . . . . . . . . . . . . . . 46

5.2 .2 Renormalization Transformation . . . . . . . . . . . 47

5.3 Fiducial Orbit . . . . . . . . . . . . . . . . . . 48

5.4 Deviation Hamiltonian . . . . . . . . . . . . . . . . 48

5.4.1 Canonical Transformation to Deviation Coordinates . . . . . . . 49

5.4 .2 Power Series Expansion . . . . . . . . . . . . . . . 49

5.5 Computational Program . . . . . . . . . . . . . . . 53

$6 \quad$ Lie Algebraic Formalism $\quad \mathbf{5 5}$

6.1 Introduction . . . . . . . . . . . . . . . . . . 55

6.2 Hamiltonian Flows and Symplectic Maps . . . . . . . . . . . 56

6.2.1 Properties of Symplectic Maps . . . . . . . . . . . . . . 57

6.3 Abstract Lie Groups and Algebras . . . . . . . . . . . . . . . 59

6.4 Lie Algebraic Tools . . . . . . . . . . . . . . . . . 60

6.4 Lie Operators and Lie Transformations . . . . . . . . . . 60

6.4.2 Useful Properties and Theorems of Lie Transformations . . . . . 62

6.5 Symbolic Computation of Maps . . . . . . . . . . . . . . . . . 64

6.6 Symplectic Numerical Integration . . . . . . . . . . . . . . . 73

$\begin{array}{lll}7 & \text { Beamline Element Description } & 77\end{array}$

7.1 Introduction . . . . . . . . . . . . . . . . . . . 77

7.2 Magnetostatic Expansion _. . . . . . . . . . . . . . 79

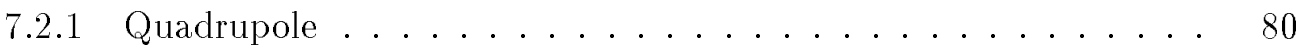

7.2 .2 Solenoid . . . . . . . . . . . . . . . . . 82

7.3 Electrostatic Expansion . . . . . . . . . . . . . . . 83

7.4 Standing Wave Electromagnetic Expansion _ . . . . . . . . . . . . 85

7.4.1 Axial Electric Field Component . . . . . . . . . . . . 87

7.4 .2 Transverse Field Components . . . . . . . . . . . . 87

7.4.3 Vector Potential Expansion . . . . . . . . . . . . . 88

7.5 Constant-z Versus Constant-t Representations . . . . . . . . . . . . . . 90 
$8 \quad$ Self-Field Impulses $\quad 93$

8.1 Introduction . . . . . . . . . . . . . . . . . . . 93

8.2 Brief Description of Particle-in-Cell Algorithms . . . . . . . . . . . . . . 94

8.3 Lorentz Force and Kick Mapping Approximation . . . . . . . . . . . . . 94

8.4 Formulation in Terms of Potentials . . . . . . . . . . . . 97

8.5 Calculation of Potentials . . . . . . . . . . . . . . . 100

8.6 Comparison with Theoretical Models . . . . . . . . . . . . . 104

9 Beam-Cavity Interaction 111

9.1 Introduction . . . . . . . . . . . . . . . . . . . 111

9.2 Fundamental Elements and Dynamics . . . . . . . . . . . . . 111

9.2.1 Modal Elements and Dynamics . . . . . . . . . . . . 111

9.2.2 Normalization of the Cavity Electric Field . . . . . . . . . . . 115

9.2.3 Periodic Klimontovich Current Distribution . . . . . . . . . . . 117

9.3 Analysis of the Circuit Equation . . . . . . . . . . . . . . 119

9.3.1 Slow Time-Scale Equation of Motion . . . . . . . . . . . . . . 119

9.3.2 Analytic Solution for Short (Slow Scale) Time Duration . . . . . 123

9.3.3 Observed Fields and Power Flow . . . . . . . . . . . . . . . . 124

9.3.4 Steady-State Behavior . . . . . . . . . . . . . . 126

9.4 Internal Bunch Dynamics and the Averaged Shunt Impedance . . . . . . 127

10 Multi-Fiducial Extensions 131

10.1 Introduction . . . . . . . . . . . . . . . . . 131

10.2 Construction of a Multi-Fiducial Map . . . . . . . . . . . . . 132

10.3 Longitudinal Beam Dynamics in an RF Cavity . . . . . . . . . . . . . 132

10.3.1 Fiducial Particle Trajectories . . . . . . . . . . . . . 133

10.3.2 Comparison of Single Versus Multiple Fiducial Calculation . . . . 133

10.4 Inclusion of Self-Field Effects . . . . . . . . . . . . . . . . . . 134

10.4.1 Split Operator Algorithm . . . . . . . . . . . . . . 134

10.4.2 Changing Representations . . . . . . . . . . . . . 135

10.5 Map Recycling . . . . . . . . . . . . . . . . . . 136

11 The RKS Code $\quad 137$

11.1 Introduction . . . . . . . . . . . . . . . . . 137

11.2 Description of the Code . . . . . . . . . . . . . . . . 137

11.2.1 Development Path . . . . . . . . . . . . . . 137 
11.2.2 Code Execution and Major Computational Cycles . . . . . . . 139

11.3 Transport in Quadrupole Channels . . . . . . . . . . . . . . . 139

11.3.1 Single Particle Dynamics and Linear Transport Matrices . . . . . 139

11.3.2 RMS Envelope Equation . . . . . . . . . . . . . . . . . 141

11.3.3 Linear Self-Field Forces . . . . . . . . . . . . . . . . . . 142

11.3.4 Quadrupole FODO Channel . . . . . . . . . . . . . . . . 143

11.4 Design of Idler and Output Cavities . . . . . . . . . . . . . . . 147

11.4.1 Steady-State Scaling . . . . . . . . . . . . . . . 148

11.4.2 Beam Energy Modulation . . . . . . . . . . . . . 150

11.4.3 Inductive Detuning for Beam Stability . . . . . . . . . . . 151

III Studies Of Beam Dynamics In RK-TBAs 153

12 Design of the RTA Experiment $\quad 156$

12.1 Introduction . . . . . . . . . . . . . . . . 156

12.2 Description of the RTA Beamline . . . . . . . . . . . . 156

12.2.1 SL4 Klystron Modulator . . . . . . . . . . . . . . 156

12.2.2 Main TBA Section . . . . . . . . . . . . . . . 158

12.3 Simulation Parameters . . . . . . . . . . . . . . . 159

12.4 Beam Matching from a Solenoid to a Quadrupole Channel . . . . . . . . 160

12.4.1 Fields and Envelopes . . . . . . . . . . . . . . 161

12.4.2 Transverse Phase-Space Evolution . . . . . . . . . . . . . 161

12.5 Longitudinal Phase-Space Evolution . . . . . . . . . . . . . . . . . 164

12.6 Transverse Phase-Space Evolution ． . . . . . . . . . . . . . . . 167

12.7 Dependence of RF Production Upon Beam Dynamics . . . . . . . . . . . 169

12.8 Extension to an Afterburner Configuration . . . . . . . . . . . . 171

12.9 Summary . . . . . . . . . . . . . . . . . . . 175

$\begin{array}{ll}\text { A RTA Project } & \mathbf{1 7 7}\end{array}$

A.1 RTA Injector . . . . . . . . . . . . . . . . . . . . . . . . . . . . . . . . 177

A.2 Pulsed Power System . . . . . . . . . . . . . . . . . 179

A.3 Injector Diagnostics . . . . . . . . . . . . . . . 180

A.3.1 Current Measurements . . . . . . . . . . . 180

A.3.2 A-K Voltage and Beam Energy . . . . . . . . . . . . . . 182

A.3.3 Current Density Profile . . . . . . . . . . . . . . 183 
A.3.4 Emittance Measurement . . . . . . . . . . . 183

B Experimental Studies in Ka-Band and Code Validation $\quad 187$

B.1 Experiments with PIVAIR at $6.7 \mathrm{MeV} \ldots \ldots \ldots \ldots$

B.1.1 PIVAIR Accelerator . . . . . . . . . . . . 187

B.1.2 Experimental Set-Up . . . . . . . . . . . . . 188

B.1.3 FEL Measurements . . . . . . . . . . . . . . . 190

B.1.4 Beam Waist Location Determination . . . . . . . . . . . . 190

B.1.5 Description of Cavities . . . . . . . . . . . . . 193

B.1.6 Cavity Measurements . . . . . . . . . . . . . 196

B.2 Simulations . . . . . . . . . . . . . . . . . . . . . 198

B.2.1 Simulation of Transport in PIVAIR . . . . . . . . . . 198

B.2.2 Simulation of Transport through the FEL . . . . . . . . . . 198

B.2.3 Simulation of Downstream Transport and Cavity Interaction . . 199

B.3 Discussion . . . . . . . . . . . . . . . . . . . . 201 


\section{List of Figures}

2.1 Proposed NLC Layout. Typical design for linear collider systems. . . . . 11

2.2 High-gradient accelerating structure. . . . . . . . . . . . . . . . 13

3.1 TBNLC waveform. . . . . . . . . . . . . . . . 25

3.2 Components in an RK-TBA beam line. . . . . . . . . . . 26

3.3 Schematic of a $2-m$ RK-TBA module. . . . . . . . . . . . . . 27

3.4 Proposed RK induction cell design. . . . . . . . . . . . . . . . . 31

3.5 Illustration of an $\mathrm{rf}$ extraction structure. . . . . . . . . . . . . 33

8.1 Loading of particles in the xy plane for a KV distribution. . . . . . . . 105

8.2 Normalized charge density (a) and electrostatic potential (b) of the KV distribution. . . . . . . . . . . . . . . 107

8.3 (a) Radial- and (b) azimuthal- electric field components of the KV distribution. . . . . . . . . . . . . . . . . 108

8.4 (a) $\mathrm{x}^{-}$, (b) $\mathrm{y}^{-}$, and (c) $\mathrm{z}^{-}$electric field components of the $\mathrm{KV}$ distribution. 109

9.1 SW $T M_{010}$ mode in a symmetric idler cavity. . . . . . . . . . . . . . 112

9.2 Relative strength of the longitudinal electric field component along the cavity's symmetry axis. . . . . . . . . . . . . . . 116

10.1 Initial fiducial coordinates and beam distribution in longitudinal phase space. . . . . . . . . . . . . . . . . . . . 133

10.2 Fiducial trajectories in an rf cavity. . . . . . . . . . . . . . . . 134

10.3 Final longitudinal beam distribution under a 3rd order map with multiple fiducials. . . . . . . . . . . . . . . . . . 135

10.4 Changing beam distribution representation from constant-z to constant-t. 136

11.1 Quadrupole gradient along FODO channel. . . . . . . . . . . . . . 140

11.2 Cosine-like (a) and sine-like (b) transfer functions. . . . . . . . . . . . 141 
11.3 RMS envelopes (a) and normalized emittance (b) evolution of a matched KV beam in a quadrupole FODO channel. . . . . . . . . . . . . . . . 144

11.4 Spurious growth in energy spread. . . . . . . . . . . . . . . . . 144

11.5 RMS envelopes of a mismatched KV beam in a quadrupole FODO channel.145

11.6 Evolution of beam modulation (a) and energy spread (b) . . . . . . . . . 146

11.7 RMS envelopes and emittance of an initially bunched beam. . . . . . . . 147

11.8 Time evolution of (a) DC bunch current and (b) bunching parameter as a function of propagation distance. . . . . . . . . . . . . . 148

11.9 Time evolution of (a) output power and (b) phase. . . . . . . . . 150

11.10Evolution of (a) average bunch energy and (b) energy spread. . . . . . 151

12.1 RTA experiment layout. . . . . . . . . . . . . . . 157

12.2 Modulation and extraction section in RTA. . . . . . . . . . . . 157

12.3 SL4 klystron gain cavities. . . . . . . . . . . . . . . . . . 157

12.4 One period of the main RTA extraction section. . . . . . . . . . . 159

12.5 Solenoid (a) and Quadrupole (b) fields in the matching section. . . . . . 161

12.6 Beam transverse RMS envelope evolution in matching section. . . . . . . 162

12.7 Observation points in the matching section. . . . . . . . . . . . . 162

12.8 Transverse phase-space evolution in the matching section. . . . . . . . . 163

12.9 Bunch current evolution from matching section through main extraction section. . . . . . . . . . . . . . . . 164

12.10Bunching parameter variation along beamline. . . . . . . . . . 165

12.11Longitudinal phase space distribution of the bunch after passing through cavities \#: 1 (a), 5 (b), 10 (c), 15 (d), 20 (e), 25 (f), 30 (g), 35 (h), and

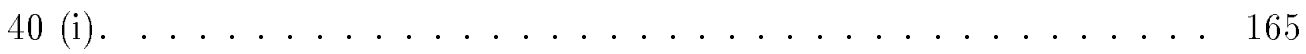

12.12Evolution of normalized RMS energy spread. . . . . . . . . . . . . . . 166

12.13Variation of bunch centroid energy along beamline. . . . . . . . . . . . 167

12.14Evolution of transverse rms envelopes . . . . . . . . . . . . . 168

12.15Evolution of transverse emittance. . . . . . . . . . . . . . 168

12.16 Transverse phase space distribution of the bunch after passing through cavities \#: 1 (a), 5 (b), 10 (c), 15 (d), 20 (e), 25 (f), 30 (g), 35 (h), and 40 (i) . . . . . . . . . . . . . . . . . . 169

12.17Transient to steady-state power output in the RTA. . . . . . . . . . 170

12.18Steady-state output power (a) and phase (b) in the main TBA section. . 170

12.19Variation of (a) bunch centroid energy and (b) normalized RMS energy spread. . . . . . . . . . . . . . . . 171 
12.20Transverse RMS envelopes (a) and emittance (b) evolution. . . . . . . . 172

12.21Transverse phase space distribution after $40 \mathrm{rf}$ cavities. . . . . . . . . 173

12.22 Current (a) and bunching parameter (b) evolution in the afterburner con-

figuration. . . . . . . . . . . . . . 173

12.23Steady-state output power (a) and phase (b) in the afterburner configuration. . . . . . . . . . . . . . . . . . 174

12.24Longitudinal phase space ( $\gamma$ vs. $c^{*}$ Arrival time ) evolution, from cavities 15 (a), 20 (b), 25 (c), 30 (d), 35 (e), 40 (f) . . . . . . . . . . 174

A.1 Schematic of the RTA injector depicting the 24 3-core cells, electrodes, glass insulators, and solenoids. . . . . . . . . . . . . 178

A.2 RTA Injector downstream. . . . . . . . . . . . . . . 181

A.3 Current measurements along the RTA beamline. . . . . . . . . . . . . 182

A.4 Injector voltage diagnostic measurements. . . . . . . . . . . . . 183

A.5 Transverse beam profile: (a) false color intensity map; and (b) intensity surface plot and gaussian best-fit. . . . . . . . . . . . . 184

B.1 PIVAIR Injector and accelerator. . . . . . . . . . . . . . . 188

B.2 Beamline layout and diagnostics for the FEL, the cavities, and beam. . . 189

B.3 Temporal evolution of beam current and output power in the FEL. . . . 191

B.4 (a) Evolution of FEL power and bunching in the SOLITUDE simulation;

(b) Experimentally measured values. . . . . . . . . . . . . . . 192

B.5 Input current waveform and distribution. . . . . . . . . . . . . . 193

B.6 Details of the standing wave rf output structures. . . . . . . . . . . . . 194

B.7 Contour lines of constant longitudinal electric field. . . . . . . . . . . 195

B.8 Cut-away view of the cavity geometry. . . . . . . . . . . . . . 195

B.9 Output current waveform and distribution. . . . . . . . . . . 197

B.10 Correlation between FEL, current, and cavity microwave output. . . . . 197

B.11 Transport in PIVAIR. . . . . . . . . . . . . . . . . . 198

B.12 Beam edge radius evolution. . . . . . . . . . . . . 200

B.13 Temporal power development in the two cavities. . . . . . . . . . . . 201 


\section{List of Tables}

11.1 Beam and cavity parameters. . . . . . . . . . . . . 147

12.1 SL4 cavity parameters. . . . . . . . . . . . . . . . . . . . . . . . . . . . . 158

12.2 Design Parameters of Main TBA Cavitites. . . . . . . . . . . . . 158

12.3 Main TBA parameters. . . . . . . . . . . . . . . . . 160

B.1 FEL parameters. . . . . . . . . . . . . . . . . . . 189

B.2 Summary of Results . . . . . . . . . . . . . . . . 196

B.3 RF cavity parameters. . . . . . . . . . . . . . . 199 


\section{Part I}

\section{Background and Overview}



Here I would like to make an analogy. There are three kinds of physicists, as we know, namely the machine builders, the experimental physicists, and the theoretical physicists. If we compare those three classes, we find that the machine builders are the most important ones, because if they were not there, we would not get into this small-scale region. If we compare this with the discovery of America, then, I would say, the machine builders correspond to captains and ship builders who really developed the techniques at that time. The experimentalists were those fellows on the ships that sailed to the other side of the world and jumped upon the new islands and just wrote down what they saw. The theoretical physicists are those fellows that stayed back in Madrid and told Columbus that he was going to land in India.

- Victor Weisskopf in The development of the concept of an elementary particle. 


\section{Chapter 1}

\section{Introduction}

Two-beam accelerators (TBAs) based upon free-electron lasers (FELs) [1] or relativistic klystrons (RK-TBAs) [2] have been proposed as efficient power sources for next generation high-energy linear colliders. Studies have demonstrated the possibility of building RK-TBAs in the X-band ( $\sim 8-12 \mathrm{GHz})$ and FEL-TBAs in the Ka-band ( $\sim 30-35 \mathrm{GHz})$ frequency regions. Provided that further prototyping shows stable beam propagation with minimal current loss and production of good quality, high-power rf fields, this technology is compatible with current schemes for electron-positron colliders in the multi-TeV center-of-mass scale.

The high intrinsic efficiency of TBAs derives from the use of induction linear accelerators (linacs). This technology is capable of generating and propagating electron beams with kiloampere peak currents, megavolt-scale energies, and pulse lengths of up to microseconds, and to do this with repetition rates stretching up to the megahertz range. Hence, the pulse format of the drive beam in the TBA can be tailored to match that of the high-energy linac, and losses in the energy conversion and transfer processes can be minimized.

TBAs must deal with extremely challenging beam dynamics. Induction linacs in general, and induction linac-driven TBAs in particular, are known to suffer from potentially debilitating transverse beam break-up (BBU) instabilities. However, it has been shown theoretically that the effect of these instabilities can be greatly decreased with appropriate design of the beamline transport and focusing system, and by tailoring some of the beam parameters.

Additionally, a strong longitudinal density modulation must be imposed upon the beam and maintained over the entire 'active' length of the TBA. Previous simulation 
efforts have shown that this is possible if the output cavities are appropriately detuned in their resonance away from the central frequency carried by the beam's modulation. As will be shown, this induces a large (up to 100\%) energy spread in the ensemble of particles comprisng the individual microbunches. The effect of this energy spread needs to be accounted for in the description of the transverse beam dynamics. It also remains to be demonstrated experimentally in a beamline of significant length.

The RTA project has been established at Lawrence Berkeley National Laboratory (LBNL) in collaboration with Lawrence Livermore National Laboratory (LLNL) to study these, and other, issues. The primary effort of this test facility is the construction of a prototype RK-TBA subunit. At the present time, the electron beam injector has been completed and is undergoing extensive testing and characterization of the emitted beam properties. Concurrently, a vigorous theoretical program is continuing to develop simulation tools and to design the remain portions of the RTA beamline.

Previous design efforts have utilized simulation codes that could only model certain aspects of the entire problem. 1-D simulations of the longitudinal dynamics in an extended relativistic klystron do not account for the transverse dynamics. 2-D simulations can not accurately model beam dynamics in both solenoid and quadrupole transport lattices. And previous 3-D simulations concentrated on modeling the physics involved in the transverse dipole BBU instability, but not the dominant rf bunch dynamics and power production in the cavity output structures. Additionally, all the previous codes have utilized a simplified model of the forces due to the self- (and image-) fields of the beam. These models do not accurately account for the details of the transverse beam distribution, and hence can not predict any changes in that distribution that may subsequently affect the interaction of the beam with the focusing lattice and rf structures.

A new simulation tool is required that can accurately model the evolution of a fully 6 -D beam distribution in non-linear and time-dependent fields. The external fields may have a mixture of solenoidal, monopole, dipole, quadrupole, and higher-order symmetry groups. Additionally, the evolution of the cavity fields must be modeled from the initial noise signal and the transient growth up to the steady-state. This code must encompass more physics than its predecessors, yet still be capable of simulating the entire experiment in a reasonable time so that it is usable as a design tool. The simulation code described here expands upon previous generations in that it captures the detailed evolution of the 6 -D phase space in the presence of nonlinear, overlapping external fields, 3-D self-field interactions, and multi-mode, time-dependent rf cavity fields. It also seeks to merge the two separate development paths, by inclusion of higher-order multipole (eg. 
dipole) modes in the beam-cavity interaction that give rise to BBU effects.

\section{Scope of this dissertation}

This dissertation is divided in three major Parts. Part I provides a general introduction to the situation in high energy physics and the issues involved with constructing high energy, high luminosity linear colliders. Then, the general topology and design principles of RK-TBAs are described in detail. These discussions have been culled and distilled from the literature, and are presented here to bring the reader 'up to speed' with the remainder of the dissertation.

Since the goal of this dissertation was to construct a new simulation code to take into account most of the relevant physical processes, Part II provides a general overview of the code. This is the heart of the dissertation, where much of the effort was made. In the development of the simulation code, several innovative methods were developed, and several well-known methods were significantly extended or improved. These are all described in detail, ending with a summary of the general architecture of the code itself.

Finally, in Part III, I discuss recent experimental and theoretical work which I have led or participated in that seeks to develop the RK-TBA concept and physical realization. First, the simulation code has been applied in the design of the RTA experiment. In this case, the code has produced a detailed description of the beam dynamics and rf power evolution over the duration of the pulse from the initial transient to the steady-state, and along the beamline from the point of initial modulation over a distance of $\sim 10 \mathrm{~m}$ and $40 \mathrm{rf}$ structures downstream. This work represents a milestone in the theoretical description of an RK-TBA, as will be discussed. Secondly, the experimental effort on the RTA injector and its diagnostics is presented. This is work in which I have directly participated, but to which I can not claim sole authorship. Lastly, the code has been used to model and design a concurrent experiment at the CEA/CESTA facility near Bordeaux, France. This effort provided the first experimental comparison with which to validate the code. In this case, I designed the cavities used in the experiment, assisted in the design of the transport beamline, and performed the simulations of the beam transport and interaction with the cavities in the region following the wiggler. I also participated in the data collection and analysis. But, again, this was a team effort. 


\section{Summary of major findings}

The design presented in Chapter 12 is the chief application for which this simulation tool was developed. Below, I summarize the main results and conclusions from that simulation.

- The transition from solenoid to quadrupole transport of an intense, bunched beam can be made with very little degradation in beam quality. The increase in emittance is only $\sim 3 \%$, with a negligible amount of current lost. Also, the longitudinal modulation (bunching) suffers no degradation during the transition.

- The lower limit in energy for a kiloamp scale RK-TBA seems to be approximately $2.5 \mathrm{MeV}$. However, the allowable normalized edge emittance is signifcantly higher than previously expected ( $1200 \pi \mathrm{mm}$-mrad instead of $800 \pi \mathrm{mm}-\mathrm{mrad})$. Hence, constraints on emittance growth during beam generation and propagation to the modulator section can be relaxed somewhat, if tighter control can be maintained once the beam is in the main TBA section.

- Larger detuning angles than previously considered (70 degrees instead of 30 degrees), and consequently larger intra-bunch energy spreads (up to $\sim 100 \%$ ), can be stably transported in the main TBA section. This has important implications for reducing low-frequency BBU via phase-mixed damping. The larger energy spread induces more rapid cycling of the synchrotron oscillation, further enhancing longitudinal stability. However, larger detuning angles induce larger differences in the bunching parameter between points of maximum and minimum compression. This results in larger variations in output power between rf cavities separated by a half-cycle of the synchrotron oscillation.

- No defocusing effects from rf cavity fields was observed. The off-axis, nonlinear part of the rf monopole fields does not seem to interfere with the permanent magnet quadrupole focusing lattice.

- The beamline design presented for the RTA experiment can, in part, be operated in the 'afterburner' mode, with only a small degradation of beam quality. This will allow a single experiment to test different segments of a complete RK-TBA system. 


\section{Topics for future studies}

The design is not yet complete, in that not every relevant piece of physics has been included. There are a small number of items that need development, implementation, or further study. A partial list is given here:

- Dipole (and higher-order) modes still need to be included in the simulation. They were not included in this discussion due to uncertainties in obtaining a reliable calculation of their mode frequencies, coupling strengths and quality factors, from a given cavity geometry. However, this may still be done with some additional effort. The effects of mode coupling and competition in a given rf cavity needs to be studied in some detail.

- The effect of element misalignment and off-axis injection of the beam can be easily modeled with this code. A parametric study for the RTA design waits to be done.

- It may be possible to model resistive wall electromagnetic modes and instabilities with a local circuit equation ${ }^{1}$. This should be done, and the influence of this effect on the stability of an intense, highly modulated beam should be studied.

- The theoretical modeling of multi-cell traveling wave structures needs to be incorporated within this framework.

\footnotetext{
${ }^{1}$ I credit William Fawley with suggesting to me this idea.
} 


\section{Chapter 2}

\section{Considerations of High Energy Linear Colliders}

\subsection{Particle Physics at the TeV Scale}

Studies of the physics of the $100 \mathrm{GeV}$ to several $\mathrm{TeV}$ mass scale is dominated by questions of the nature of electroweak symmetry breaking [3] [4]. The Standard Model of the strong, weak, and electromagnetic forces has shown phenomenal predictive power at lower mass scales, and makes concrete predictions of the dominant processes at higher mass scales as well. However, there are some basic philosophical problems with this model that lead physicists to suppose that a new regime of fundamental particles and their interactions lie beyond the currently accessible mass scale. But, without addressing these new mysteries, there still remain some unanswered questions in the Standard Model. The Higgs mechanism plays a crucial role by giving mass to the $\mathrm{W}$ and $\mathrm{Z}$ gauge bosons as well as to the Standard Model fermions. The mass of the Higgs boson itself should lie near the scale of electroweak symmetry breaking. Thus, finding the Higgs boson (or bosons in supersymmetric extensions to the Standard Model), and performing detailed studies of its interactions, is of principal importance in high energy physics.

To probe this scale in a laboratory setting, new high energy collider facilities have been proposed or are currently under construction. The Tevatron, LEP, and SLC have been the primary tools over the last decade in probing the highest mass scales. While there may be a possibility of finding a light Higgs boson at either LEP or the Tevatron [5] in the 100-200 GeV range, the Large Hadron Collider (LHC) at CERN with a center-ofmass collision energy of $7 \mathrm{TeV}$ will extend the reach of searches to the $1 \mathrm{TeV}$ mass scale 
range. Future electron-positron linear colliders in the $500 \mathrm{GeV}$ to several $\mathrm{TeV}$ center-ofmass energies, as well as the more exotic muon-muon colliders, are currently under design to provide a laboratory environment for detailed study of the particle interactions once a new signature has been found. Additionally, lepton colliders provide complementary search pathways to that of hadron colliders [3] by utilizing different production and decay channels.

This dissertation deals exclusively with some aspects of the technology which can be used in electron-positron linear colliders. We look at the features of these colliders in more detail.

\subsection{Linear Collider Physics at the TeV Scale}

An example of a future linear collider design is provided by the proposed Next Linear Collider (NLC) [4]. A layout is shown in Figure 2.1. The general topology of many linear collider systems is based on the current model, the Stanford Linear Collider (SLC) [6], with some important differences. There are two arms of the collider; one for electrons and the other for positrons. Each arm includes an electron source and injector (or source complex for positron production); a damping ring to compress transverse phase space volume through radiation and re-acceleration processes; a sequence of bunch length compressors to minimize head-tail energy variations over the beam bunches; a main linear accelerator (linac) to boost the collision center-of-mass energy; and a final focus section to increase luminosity. The two arms then meet at a single interaction point (IP). In some designs, there are additional IP's for gamma-gamma, gamma-electron, and gamma-positron collision studies.

\subsubsection{Interaction Point}

At the interaction point (IP) we see an intersection of beam physics and machine performance with the expected occurence rates of interesting processes. The ability to produce many interesting processes above the background, in a reasonable time, becomes a figure of merit for any collider system. This figure is called the luminosity of the machine.

The geometric luminosity function of any collider system is given by the relation

$$
L_{0}=\frac{n N^{2} f_{r e p}}{4 \pi \sigma_{x}^{*} \sigma_{y}^{*}}
$$

where $N$ is the number of electrons and positrons in each colliding bunch; $n$ is the 


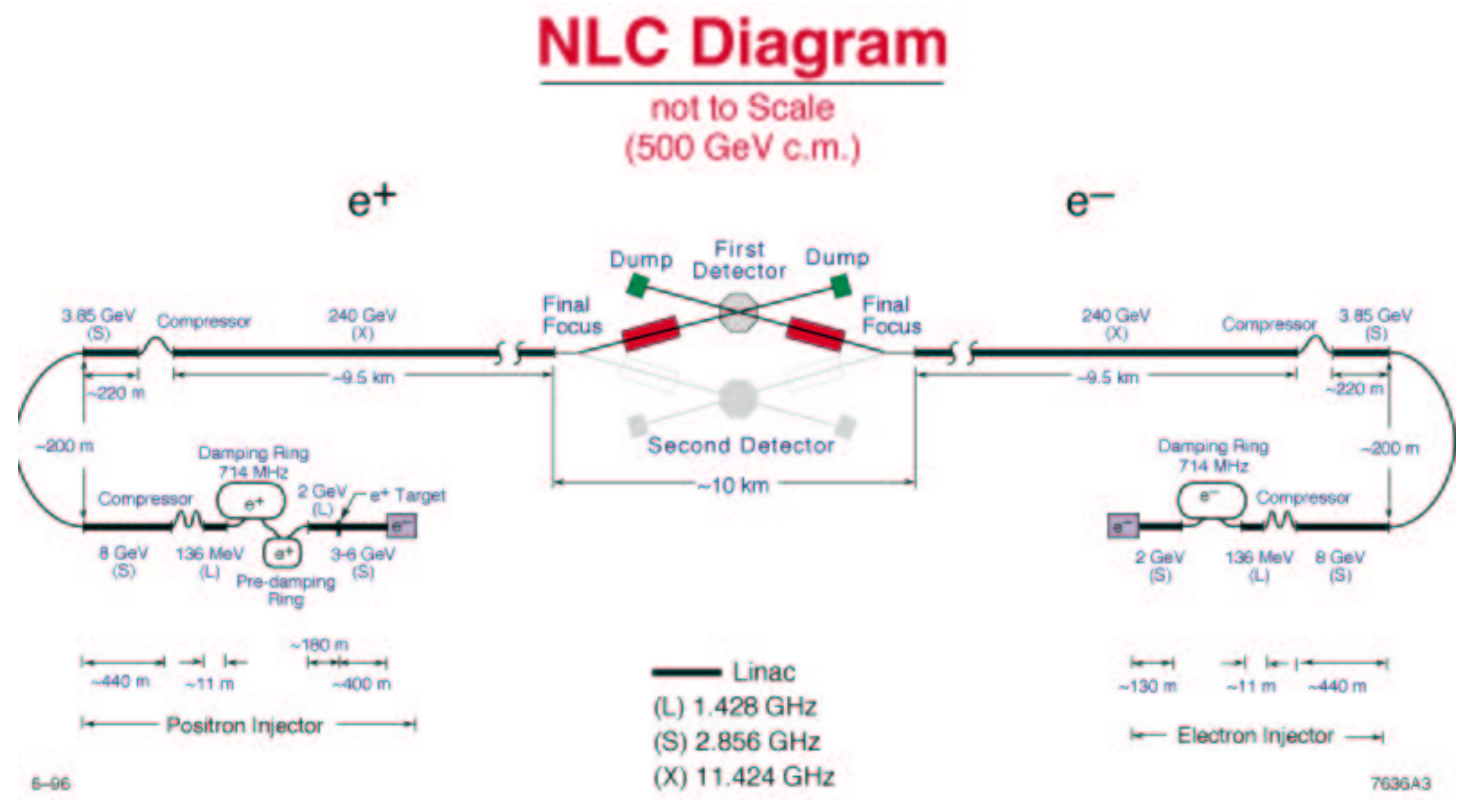

Figure 2.1: Proposed NLC Layout. Typical design for linear collider systems.

number of bunches in each pulse train; $f_{r e p}$ is the pulse repetition frequency; $\sigma_{x(y)}^{*}$ is the horizontal (vertical) size of each bunch at the collision point, determined by the optics of the final-focus magnets. We express the total luminosity as a product of the geometric luminosity and the pinch enhancement factor, $H_{D}$. That is,

$$
L=L_{0} H_{D}
$$

This additional factor accounts for the dynamical evolution of the colliding bunches as they experience the intense electromagnetic fields of each other. This interaction has a number of practical consequences.

The first is the phenomenon of disruption. The fields of the charges particles in the oncoming bunch can act as a lens and produce a focusing effect [7][8][9][10]. If the focal length is large compared to the bunch length $\left(\sigma_{z}\right)$, the effect is small and tends to produce a pinch of the transverse beam size, thus enhancing the luminosity by decreasing the effective product $\left\langle\sigma_{x}\right\rangle\left\langle\sigma_{y}\right\rangle$. On the other hand, if the effect is large and the bunch length is much greater than the focal length, the bunch will undergo strong oscillations in its transverse dimensions along the length of the bunch that tend to disrupt the effect of the final-focus magnets and reduce the luminosity. This is a collective effect that contrains the beam intensity $(N)$ and the transverse and longitudinal dimensions of the 
bunch.

Next, the electromagnetic interaction of the particles within a given bunch with the collective fields of the incoming bunch will alter the trajectories of these particles. The particles will emit synchrotron radiation, conventionally called beamstrahlung [7][9]. As a first-order interaction, this phenomenon has the dual effect of increasing the soft and hard electromagnetic background as well as increasing the energy spread of the particles in the colliding bunches. Following bunches in the closely-spaced bunch trains may then also suffer due to this background. To higher-order, interactions between the incident electrons/positrons and these photons may increase the production of low energy hadrons, hadronic jets, electron/positron pairs, etc. in the overall background.

Attention to the beam physics at the interaction point produces many constraints on the beam generation, acceleration, and delivery systems. The beam's energy, transverse and longitudinal dimensions, and energy spread must be kept within strict tolerances. The luminosity and the beam-beam interaction depend upon certain properties of the beam at the interaction point and the dynamics during the collision process. These parameters are independent of the details of the acceleration process and hardware. An additional parameter, independent of the linac, is the average beam power, $P_{b}=$ $n N f_{r e p} E_{b}$, where $E_{b}$ is the beam energy. The luminosity can then be expressed in the more transparent form

$$
L=\frac{1}{4 \pi E_{c m}} \frac{N H_{D}}{\sigma_{x}} \frac{2 P_{b}}{\sigma_{y}}
$$

where $E_{c m}=2 E_{b}$ is the center-of-momentum collision energy. This form of the luminosity function clearly demonstrates the related dependencies [10]. The first factor is fixed by the scale of the physics being probed. The second factor is determined by the beam-beam interaction, and the amount of background processes tolerated by the detector systems. These two factors are typically set before the details of the collider can be determined, and act as severe constraints. The third factor, then, is the only way that the details of the collider design will enter the calculation of the luminosity. Taken by itself, this factor represents a tradeoff between our ability to focus intense beams to arbitrarily small spot sizes and our ability to handle beams carrying arbitrarily large power. The spot size, $\sigma_{y}$, has been argued [10][11] to depend primarily upon our ability to damp vibrational motion and to correct for misalignments down to the level of 10 's of picometers.

It is in the factor of the beam power that we (as designers) have the most liberty. 


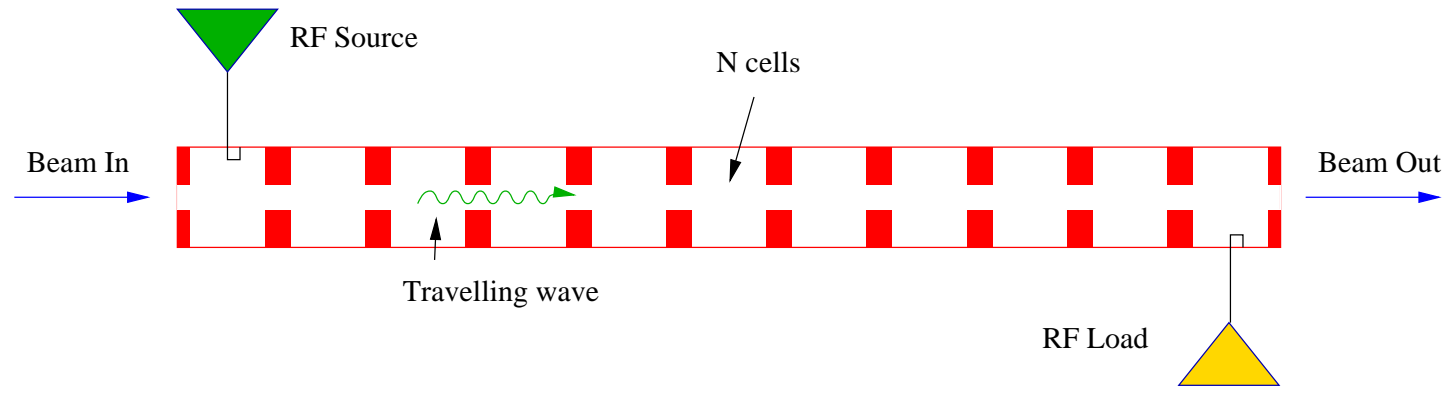

Figure 2.2: High-gradient accelerating structure.

While the beam power is independent of the linac, the individual factors used to define the beam power are not. This has led to a number of different strategies and proposals for next generation electron-positron colliders. These include the TESLA design using superconducting cavities in the main linacs [12], the NLC/JLC [13] proposal using room temperature copper cavities at X-band $(11.424 \mathrm{GHz})$ frequencies, the CERN CLIC [14] two-beam accelerator using Ka-band $\left({ }^{\sim} 30 \mathrm{GHz}\right)$, and others. Updated comparisons of the parameters for these various collider designs has been compiled by International Linear Collider Technical Review Committee [15].

\subsubsection{High-Gradient Linac and RF Power Source}

A schematic of a typical accelerating structure is shown in Figure 2.2. This is an example of a periodic, iris-loaded waveguide structure with $N$ cells that can act as a linear accelerator [16] [17] [18] [19] [20]. An RF source provides the feed for the fields that accelerate the beam. These fields are arranged into a travelling wave mode that propagate to the end of the structure, at which point they are dumped into an impedance-matched load to minimize reflection. Energy from this wave is absorbed by the co-propagating beam, as well as by the finite-conductivity walls of the structure.

This system of RF sources and accelerating structures functions by converting AC power to usable beam power with as high an efficiency as possible. The design of the system must proceed by creating the desired accelerating gradient with some fraction of the input RF power from the source, and then to transfer some fraction of this power to the beam as it traverses the structure.

A constant impedance (CZ) structure is characterized by a uniform group velocity of the travelling RF pulse and attenuation per unit length along the structure. This is the simplest type of accelerating structure. The radius of the waveguide, the iris thickness 
and aperture radius, and cell length are all held constant. This is in contrast with constant gradient (CG) structures which allows for variations in cell-to-cell geometry so that the group velocity $\left(v_{g}\right)$ and attenuation $(\alpha)$ vary along the structure. This permits the structure to present a uniform accelerating gradient along the entirety of its length to the beam. Detuning the cells from each other slightly has the beneficial effect of decohering higher-order travelling dipole fields that lead to cumulative beam breakup [21] [22]. However, for the purpose of examining issues of power flow and scaling behavior, we will concentrate here only on $\mathrm{CZ}$ structures.

A third mode of energy loss in structures is due to the phenomenon of beam loading. From general considerations of reciprocity, a beam can also serve as a source that drives fields in the accelerator structure. The fields emitted by the beam bunches as they traverse the structure are collectively called the bunch wake. For the bunch emitting the wake, this provides an energy loss mechanism that must be considered in any calculation of the net accelerating gradient of the structure. For single particles, the wake is due primarily to the geometry of the structure, and in the coupling of the mode to the particle trajectory. This is also the case for the fields of the actual accelerating mode, demonstrating the maxim that good accelerators are good decelerators [19].

After an initial period of transient loading of the structure, the fields reach a steadystate condition. In the limit of a large number of cells in the structure $(N>>1)$, the accelerating gradient $\left(G_{s}\right)$ seen by the beam per unit length has the behavior [9]

$$
\frac{d G_{s}}{d z}=-\alpha G_{s}-I_{0} r \alpha
$$

where $I_{0}$ is the DC current carried by the beam; $r$ is the shunt impedance per unit length which measures the coupling of the beam to the mode; $\alpha=\frac{\omega}{2 v_{g} Q_{w}}$ is the attenuation per unit length of the fields with angular frequency $\omega . Q_{w}$ is the quality factor of the mode, defined as $Q_{w}=\frac{\omega u}{P_{1}}$, where $u$ is the stored energy per unit length and $P_{1}$ is the peak-power dissipation per unit length. The attenuation parameter of the structure (with length $L$ ) is given by $\tau=\alpha L=\frac{T_{\text {fill }}}{T_{\text {decay }}}$ with the fill-time defined by $T_{\text {fill }}=L / v_{g}$ and the decay-time from wall losses defined by $T_{\text {decay }}=2 Q_{w} / \omega$.

The peak input RF power, $P_{0}$, is related to the peak accelerating gradient, $G_{0}$, via

$$
P_{0}=\frac{G_{0}^{2}}{2 \alpha r}
$$

and provides an initial condition to (2.1). Integrating (2.1), we obtain the variation of 
accelerating gradient with longitudinal position including beam loading effects,

$$
G_{s}(z)=G_{0} \exp (-\alpha z)-I_{0} r[1-\exp (-\alpha z)]
$$

The power flowing in the structure at any point is then given by $P_{s}(z)=G_{s}^{2} /(2 \alpha r)$. At the end of the structure $(z=L)$, the gradient has the finite value

$$
G_{L}=G_{0} \exp (-\tau)-I_{0} r[1-\exp (-\tau)]
$$

and the remaining power $\left(P_{S L}\right)$ flows into the matched load. The fraction of the input power deposited into the load is given by

$$
\eta_{S}=P_{S L} / P_{0}=\left(G_{L} / G_{0}\right)^{2}=[\exp (-\tau)-\chi(1-\exp (-\tau))]^{2}
$$

with $\chi=I_{0} r / G_{0}$.

The power delivered to the beam during its interaction with the structure is $P_{b}=I_{0} V$, where $V$ beam-loaded voltage gain

$$
V(z)=\int_{0}^{z} d z^{\prime} G_{s}\left(z^{\prime}\right)
$$

Performing this integration we obtain the power absorbed along the structure length

$$
P_{b}(z)=\left(I_{0} G_{0} L+I_{0}^{2} r L\right)\left(\frac{1-\exp (-\alpha z)}{\tau}\right)-I_{0}^{2} r L
$$

and the net absorbed power at $z=L$ is

$$
P_{b L}=I_{0} G_{0} L\left[(1+\chi)\left(\frac{1-\exp (-\tau)}{\tau}\right)-\chi\right]
$$

The net efficiency of extraction is then

$$
\eta_{b}=P_{b L} / P_{0}=2 \chi(1+\chi)(1-\exp (-\tau))-2 \chi^{2} \tau
$$

Additionally, by averaging the beam-loaded gradient (2.3) over the length of the structure we obtain

$$
\bar{G}=G_{0}\left[(1+\chi)\left(\frac{1-\exp (-\tau)}{\tau}\right)-\chi\right]=V(L) / L .
$$

This last quantity in brackets is the inverse of the peak field enhancement factor which relates the peak on-axis fields in the structure to the average accelerating gradient, and 
is an important quantity in determining RF breakdown due to field emission.

The power dissipated into the walls is determined from

$$
\frac{d P_{w}}{d z}=2 \alpha P_{s}
$$

We may easily integrate this equation to obtain the total power loss normalized to the input power. The fraction of power absorbed by the walls is then

$$
\eta_{w}=(1+\chi)^{2}(1-\exp (-2 \tau))-4 \chi(1+\chi)(1-\exp (-\tau))+2 \tau \chi^{2}
$$

It is easily verified that $\eta_{s}+\eta_{b}+\eta_{w}=1$, as required from conservation of energy principles.

These considerations of efficiencies can point the linac designers in particular directions, depending on what technologies they are more familiar with, and upon which they feel more comfortable placing additional burdens. In the case of an RK-TBA-driven linear collider, it has been argued [23] that the intrinsic high efficiency of the RF source allows the use of very high power beams in the main linacs, while loosening some of the more stringent parameters in the final focus section. In this case, the high beam power in the linac is carried through a high average current. The accelerating structures are designed to be highly efficient in the transfer of RF power to beam power ( $\sim 80 \%)$, with high input RF power. These structures operate in a regime of heavy beam loading, with negligible amounts of power ending up in the matched load.

\section{Limits to gradient}

Reaching high gradients in accelerating structures is limited by certain phenomena: field emission, dark current capture, RF breakdown, and pulsed heating. Field emission refers to the phenomenon of extracting electrons from a surface in the presence of a large, local electric field [24]. Microscopic irregularities, protusions, and sub-surface dielectric or metallic inclusions can enhance the actual local field to many times that of the theoretical surface field values. Emitted electrons introduce a noticeable current when the local field gradient is one the order of a few $\mathrm{GV} / \mathrm{m}$ [25]. A typical value of the local field enhancement in common disk-loaded structures is approximately 50 , so that the applied macroscopic surface field corresponding to this emission is roughly 40 $\mathrm{MV} / \mathrm{m}$. For these structures the ratio of the on-axis gradient to the peak surface field is approximately $1 / 2$, leading to a peak accelerating field of $20 \mathrm{MV} / \mathrm{m}$ before field emission 
from surfaces becomes noticeable. Aggressive cleaning and high-power RF processing can be used to reduce some of the risk of unintentional emission.

At reduced power levels, the field emitted electrons follow somewhat random trajectories from the emitting surface to the lower-field surfaces. On impact, they can produce lower-energy secondaries. Together, these electron form a kind of gas inside the accelerating structures [26]. As the accelerating gradient is increased past a threshold value [27], $G_{t h}$, where

$$
G_{t h} \lambda_{r f}=1.6 M V
$$

the emitted electrons may be channeled and captured by the accelerating and transport fields [28]. This current may then increase from structure to structure along the length of the linac. These low energy electrons constitute the so-called dark current, which can increase detector backgrounds, parasitically absorb RF energy in the structures, and may disrupt the main beam. The scaling law above implies that higher gradients may be obtained by moving to higher operating frequencies of the RF fields.

$R F$ breakdown is an instability in the cavities that arises at high levels of stored energy. This effect is also due to field emitted electrons, though the exact mechanism is not yet known exactly. The onset of a breakdown is characterized by coincidental observations: (i) an abrupt increase in the reflected RF signal from the cavity due to a sudden shift in loading and Q-values; (ii) an increase in prompt x-ray emission; and (iii) a degradation of the vacuum. Experimental work over the last half-century has attempted to measure the onset of RF breakdown in structures with increasing field gradients [26] [29] [30]. Current conservative estimates of the dependence of the maximum macroscopic surface fields upon operating frequency yield the empirical fit

$$
E_{s}=220[f(G H z)]^{1 / 3} M V / m .
$$

This scaling, again, shows that increased gradients can be tolerated by using higher operating frequencies.

Pulsed heating refers to the deposition of thermal energy by Ohmic loss in the structures walls during a single pulse of RF energy [31] [32]. A diffusion process describes the flow of heat in the structure walls. The heat flow produces lateral expansion of the cavity surfaces that induces mechanical stress. Repeated cyclic stress may then fatigue the cavity walls, producing microcracks and others deformations, which eventually degrade the performance of the structure. During the very short times during which heat is deposited in the cavity walls the walls will not expand elastically, but are susceptible 
to plastic deformation. To stay below this threshold, the temperature rise during the pulse must maintained below some critical value ${ }^{1}$. For a given temperature increase, this limits the input power to the cavity, and hence the maximum accelerating gradient. A convenient scaling is given by Wilson [33]

$$
\Delta T \propto G^{2} \lambda_{r f}^{1 / 4}
$$

Hence, for a given maximum temperature change, $G_{\max } \propto \lambda_{r f}^{-1 / 8}$. This is a severe limit on the attainable maximum gradient in room-temperature copper accelerating structures. However, these limits are still higher than what can currently be attained. For a set temperature rise of $150 \mathrm{~K}$, the maximum gradient at $40 \mathrm{GHz}$ is $\sim 300 \mathrm{MV} / \mathrm{m}$, which increases to $\sim 330 \mathrm{MV} / \mathrm{m}$ at $91.4 \mathrm{GHz}$ [31]. Currently proposed colliders [15] are based upon unloaded gradients in the range of $20-50 \mathrm{MV} / \mathrm{m}$ in the $\mathrm{S}$ - and $\mathrm{X}$-Bands $\left({ }^{\sim} 2-12\right.$ $\mathrm{GHz})$, and $80 \mathrm{MV} / \mathrm{m}$ in $\mathrm{Ka}-\mathrm{Band}\left({ }^{\sim} 30-35 \mathrm{GHz}\right)$, well below this pulsed heating limit.

Issues and phenomenon related to field emission force us to higher operating frequencies in order to raise to the accelerating gradient. And, even assuming that we are not so bold as to cross the pulsed heating boundary, there is another limitation that we must address. The stored energy per unit length in the accelerating structure will scale with frequency and gradient as

$$
u_{\text {stored }} \propto G^{2} \lambda_{r f}^{2}
$$

Setting the gradient as some constant factor multiplying the dark current instability threshold value, the stored energy per unit length becomes roughly constant and frequency independent. The decay time, $T_{\text {decay }}=2 Q_{w} / \omega$, scales as $f_{r f}^{-3 / 2}$ and hence the $\mathrm{RF}$ pulse length delivered by the source will also tend to scale as $f_{r f}^{-3 / 2}$. However, since the stored energy per unit length is roughly constant, the peak RF power per unit length of structure must necessarily scale as $f_{r f}^{3 / 2}$.

This scaling runs opposite to what is possible with conventional klystron technology [34]. To increase peak output of the RF source, some sort of pulse compression system must be employed alongside conventional klystrons. This introduces new loss mechanisms and the net efficiency of the RF source will decrease. Another method of obtaining the requisite peak power (100's of MW to GW scale) with the short pulse lengths (10's 100 's of nanoseconds) in the $\mathrm{X}$ - and Ka-band operating frequencies is to use relativistic

\footnotetext{
${ }^{1}$ For various grades of copper and copper alloys, the tolerable temperature rise sits in the approximate range of $40-240$ degrees $\mathrm{K}$
} 
RF sources.

\section{Power Sources Based on Linear Induction Accelerators}

Linear induction accelerators (induction linacs) [35] are a proven technology for producing multi-gigawatt electron beams with pulse formats compatible with the needs of high-gradient accelerating structures for linear colliders. They are inherently efficient $(\geq 60 \%)$ at converting $\mathrm{AC}$ power to beam power over pulse lengths of 10 's to 100 's of nanoseconds [36].

Experiments at Lawrence Livermore National Laboratory (LLNL) during the 1980's and 90 's investigated the application of induction linacs to the problem of generating high quality RF power for colliders. The ELF free-electron laser (FEL) experiment [37] [38] generated $1 \mathrm{GW}$ of power at $34 \mathrm{GHz}$ with $40 \%$ efficiency in the conversion of beam power to RF, and relativistic klystron (RK) experiments in collaboration with Stanford Linear Accelerator Center (SLAC) and Lawrence Berkeley National Laboratory (LBNL) [39] [40] [41] yielded $330 \mathrm{MW}$ at $11.4 \mathrm{GHz}$ from a velocity-modulated beam, and 400 MW at $11.4 \mathrm{GHz}$ from a transversely-modulated beam.

Two-beam accelerators (TBA's) based on induction linacs and either FEL's [1] (FELTBAs) or RK's [2] (RK-TBA's) were proposed to take advantage of the inherent efficiency of the acceleration process as a way to boost the overall efficiency of the RF source. In this concept, the beam producing the RF fields is re-accelerated after a small fraction of its kinetic energy is extracted in the form of RF fields. By continuing to re-accelerate and re-extract power, the inefficiencies from the beam generation, beam head losses, and eventual termination in the beam dump are amortized over a much greater number of interactions and extraction regions, and the net efficiency increases.

There are important differences between TBAs based upon FELs and those based upon relativistic klystrons, and this leads to a natural choice between these two technologies for driving high gradient linacs at different frequencies. In linear induction linacs, the size of the beam pipe is typically determined by transverse instabilities. The beam break-up (BBU) instability arising from the transverse impedance of the accelerating gap varies as the inverse square of the pipe radius. In a collider-scale TBA of kilometer

length, to keep the growth of this instability to tolerable levels and enable stable beam transport, the pipe radius must be larger than approximately one centimeter. To construct an RK-TBA, based on power extraction from resonant cavities, the beam tubes leading into and out of the individual rf cavities must have a radius below that of the cutoff-radius in order to prevent the mode from escaping. Balancing these two consid- 
erations, one can see that RK-TBAs lose feasibility at frequencies above approximately $40 \mathrm{GHz}$. For frequencies above this point, we are driven to consider beam-wave interactions that can produce good quality, high-power rf in overmoded waveguides. This has been shown by using free-electron lasers, although interactions based upon cyclotron autoresonant masers (CARMs) are also possible. However, these beam-wave interaction devices suffer from problems in the phase stability of the output rf power that devices based upon beam-cavity interactions do not. It thus presents an easier task to produce high-quality rf power from an RK than an FEL. Now, the majority of linear collider systems currently under study are based around operating frequencies in the bands spanning from $11 \mathrm{GHz}$ to $35 \mathrm{GHz}$. In this regime, the RK-TBA is the obvious choice for a TBA form of power source.

A relativistic klystron two-beam accelerator (RK-TBA) can be envisioned to span kilometer-scale distances and to provide RF power for 10's to 100's of high-gradient accelerating structures. Previous re-acceleration experiments [42] have demonstrated successful transmission of a high-power electron beam through two induction cells and three RF extraction cavitites. Total output RF power of $200 \mathrm{MW}$, with phase and amplitude stability over a significant portion of the beam pulse was shown,

\section{Efficiencies}

The power absorbed by the beam originates on the power supply grid and must be transformed through a series of steps. Each step on this process introduces some loss, and must be accounted for in the calculation of the net efficiency. The net efficiency relates the $\mathrm{AC}$ wall-plug power to the final beam power. This net efficiency can be factored in to distinct, cascading parts

$$
\eta_{\text {wall }->\text { beam }}=\eta_{A C->D C} \eta_{D C->R F} \eta_{R F->\text { beam }}
$$

The first factor, $\eta_{A C->D C}$, is the conversion efficiency of AC power in DC power supplies. This value is typically in the $90-95 \%$ range. The second factor, $\eta_{D C->R F}$, is the net efficiency of producing RF fields and transporting them to the accelerating structure. This term includes any efficiencies in handling DC pulsed-power, the RF source and associated systems, and peak RF power handling [15]. The final term, $\eta_{R F->b e a m}$, is the net efficiency of power transfer from the structure to the beam, (2.4). The technologies and strategies of the various collider systems involve trade-offs between achievable efficiencies in producing beam power and the resulting bunch parameters at the IP. These 
trade-offs have been discussed for RK-TBAs in the context of multi-TeV linear colliders [23] [43]. 


\section{Chapter 3}

\section{Relativistic Klystron Two-Beam Accelerators}

Two-beam accelerators based upon the relativistic klystron concept have been proposed as drivers for high-gradient accelerating structures in the frequency range from 11-40 $\mathrm{GHz}$. This corresponds to linear collider center-of-mass energies from $1 \mathrm{TeV}$ to $5 \mathrm{TeV}$. The main components and design philosophy can be gleaned from examining the point design for a system that could act as an upgrade in energy and luminosity for the NLC. This system is dubbed the 'TBNLC'.

\subsection{Power Source Requirements for an NLC Upgrade to 1.0 $\mathrm{TeV}$}

The objective of the TBNLC system study was to construct a conceptual design of the power source for the NLC. Our philosophy was to stay within the design constraints of the NLC and to have a power source system that matches the high gradient structures being considered for the NLC. However, since the parameters of the NLC were not finalized at the time of the study, we made some choices for "power source requirements" so that the design and costing studies could be concrete. The design goals for the study were as follows:

- RF frequency: $11.424 \mathrm{GHz}$

- Repetition rate: $120 \mathrm{~Hz}$

- Peak power/structure: 360 MW 
- Distance between extraction structures: $2 \mathrm{~m}$

- Pulse length (flat-top): $200 \mathrm{~ns}$

- Pulse rise time: $100 \mathrm{~ns}$

The peak power of $360 \mathrm{MW}$ at $11.424 \mathrm{GHz}$ corresponds to an unloaded gradient of 100 $\mathrm{MV} / \mathrm{m}$ in the NLCTA high gradient structures tested at SLAC. This power is generated from an extraction cavity in the RK every $2 \mathrm{~m}$, to match the $1.8 \mathrm{~m}$ accelerator sections, and to provide adequate spacing in between the HGS's for input and output couplers, focusing, etc. In this study, we examine the power source for a total of $15 \mathrm{~km}$ of this high gradient structure, $7.5 \mathrm{~km}$ for each arm of the collider. There is a total of 7500 rf extraction cavities, with a total peak power of $2.7 \mathrm{TW}$. The unloaded energy gain from each arm of the collider is $675 \mathrm{GeV}$. Assuming a loaded gradient that is $75 \%$ of the unloaded, final energies of the electron and positron bunches are $506 \mathrm{GeV}$ each. The pulse length was determined by the fill time of the structure plus the length of the multi-bunch particle train. In order to maintain constant particle energy from bunch to bunch, the required electric-field should grow linearly in the first $100 \mathrm{~ns}$, and remain constant over the remainder of the beam-on time. The flat-top is specified as $200 \mathrm{~ns}$, approximately twice the flat-top pulse length of the present parameter list ( $\sim 100 \mathrm{~ns}$ flat-top), which allows us to accelerate pulse trains of 143 bunches and leads to a corresponding increase in luminosity. This is possible for our TBA design, and is a natural extension of the NLC parameter set to take advantage of the RK-TBA structure. Because of the intrinsically high efficiency of the RK-TBA, the average power required stays at about $200 \mathrm{MW}$. The required electric field at the front of the $100 \mathrm{~ns}$ rise is roughly $25 \%$ of the asymptotic value. Hence, we specify our input pulse to rise linearly (in field) from zero to its full value in $100 \mathrm{~ns}$, shown in Figure 3.1. The power increases quadratically over the rise time. This pulse shape can be generated in an RK by a corresponding linear rise in the current waveform of the drive beam, and a linear rise in the reacceleration voltage as well. Further refinement of the pulse shape to match dispersion in the high-gradient structure and to improve energy flatness can be achieved by appropriate shaping of the drive beam current and voltage.

\subsection{TBNLC Architecture}

To provide rf power for a 1.5- TeV high gradient linear accelerator, the TBNLC design requires 76 independent RK-TBA units. Each RK-TBA unit is about $300 \mathrm{~m}$ long, and 


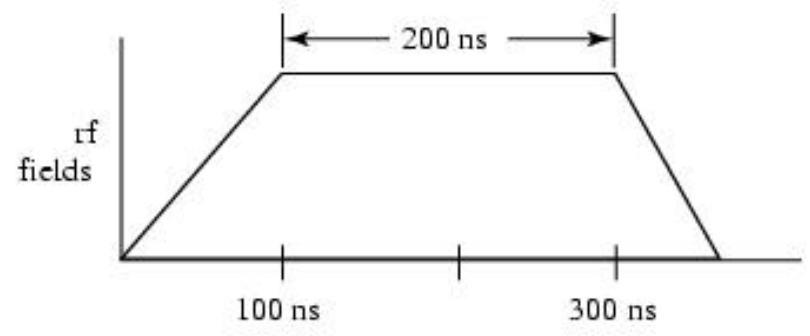

Figure 3.1: TBNLC waveform.

has 150 extraction cavities (shown in Figure 3.2). To replenish the $360 \mathrm{MW}$ generated from the extraction cavity every $2 \mathrm{~m}$, the $10 \mathrm{-MeV}$ drive beam with average current of $600 \mathrm{~A}$ is reaccelerated at $300 \mathrm{kV} / \mathrm{m}$. The design current could be raised slightly to compensate for rf losses in the induction cells (e.g. $625 \mathrm{~A}$ for $4 \%$ loss). The main body of the RK consists of identical 2-m modules each of which has six $100 \mathrm{kV}$ induction cells and one extraction cavity. Both the drive beam current and reacceleration voltage have a rise time of $100 \mathrm{~ns}$ and a $200 \mathrm{~ns}$ flat-top, with a fall time that is comparable to the rise time.

The front end of each RK consists of an injector in which a 1.2-kA beam of electrons is generated and accelerated to $2.5 \mathrm{MeV}$. A 5.7- $\mathrm{GHz}$ chopper is placed at this point to generate a bunched beam at $11.4 \mathrm{GHz}$. Chopping reduces the dc current from $1.2 \mathrm{kA}$ to $600 \mathrm{~A}$. This bunched beam at $2.5 \mathrm{MeV}$ is then accelerated to $10 \mathrm{MeV}$ in an 'adiabatic compressor' section. Bunching cavities in this section further reduce the length of the bunches, and prepare the beam with the right phase space for injection into the main RK. At the end of the RK, there is an 'afterburner' section in which rf extraction continues, but reacceleration is absent. The afterburner allows us to further extract rf power from the bunched beam, and thus enhances the overall efficiency of the RK. At the end of the afterburner, the spent beam (at 2 to $3 \mathrm{MeV}$ ) is collected at a beam dump. The adiabatic compressor section is $26 \mathrm{~m}$ long, while the main RK has 138 extraction cavities over $276 \mathrm{~m}$. The afterburner consists of $12 \mathrm{rf}$ cavities, making a total of 150 extraction cavities. The overall length of a RK-TBA unit is determined by a balance of two opposing considerations. The longer the RK-TBA unit, the higher the overall efficiency, as the overhead losses from the front and the back ends become a 


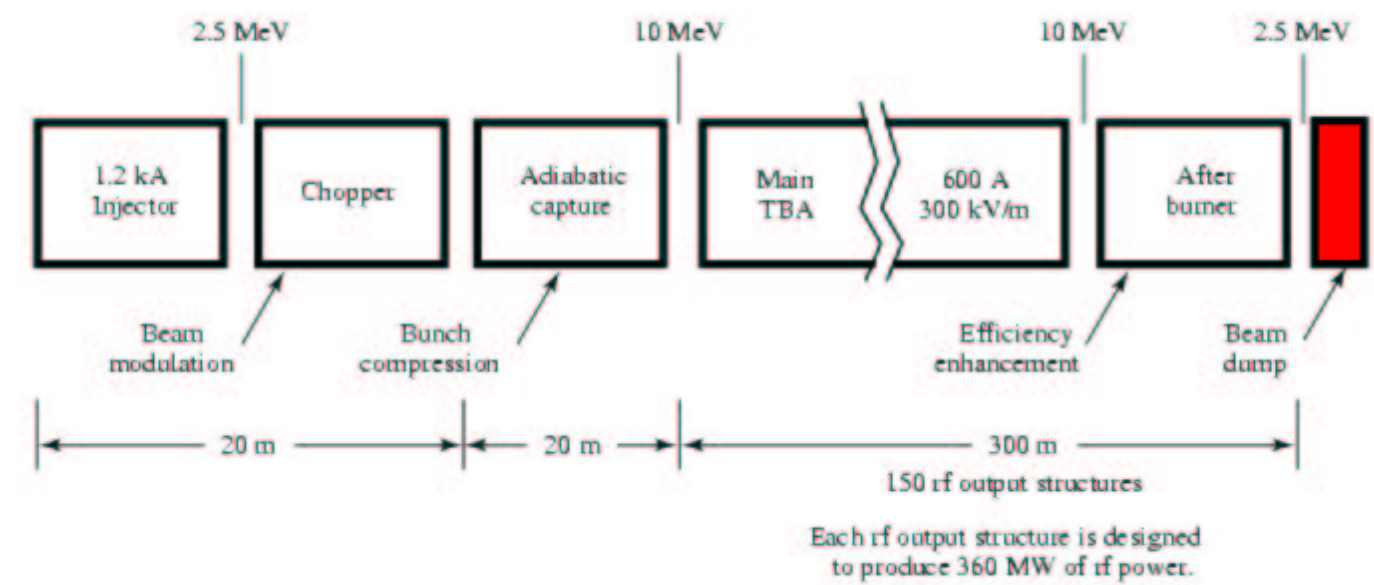

Figure 3.2: Components in an RK-TBA beam line.

smaller fraction of the total power. On the other hand, the control of beam instabilities and beam degradation effects become increasingly more difficult as the overall length is increased. Longitudinal and transverse beam dynamics simulations indicate that the stability of the bunched beam can be maintained in the proposed RK-TBA unit. The efficiency for conversion of power in the drive beam to rf power is easily evaluated for the proposed RK system. Allowing for some rf loss in the induction cavities, the overall efficiency is estimated to be $90 \%$. The $10 \%$ loss is shared among the beam loss on the chopper $(3.7 \%)$, beam dump $(2.8 \%)$ and rf into induction cavities $(3.6 \%)$. The efficiency for wall plug to drive beam is $55 \%$. Hence the total efficiency from wall plug to rf is $50 \%$. The rf power requirement of $360 \mathrm{MW}$ per 2 meters determines the product of the accelerating gradient and beam current in the drive beam. Our particular choice of $300 \mathrm{kV} / \mathrm{m}$ and $600 \mathrm{~A}$ is again based on a balance of two considerations. With higher current and lower gradient, the volume of magnetic material in the induction modules required is reduced and the efficiency is increased. However, beam transport becomes more difficult with increasing current. The linear rise in the drive beam current assures that the extracted rf field has the right waveform for beam loading in the HGS, while the linear rise in the voltage of the reacceleration cells assures that the entire drive beam stays at $10 \mathrm{MeV}$ from head-to-tail. This is of course important for both beam transport as well as for rf phase stability. Hence, the rising portion of the current and voltage 


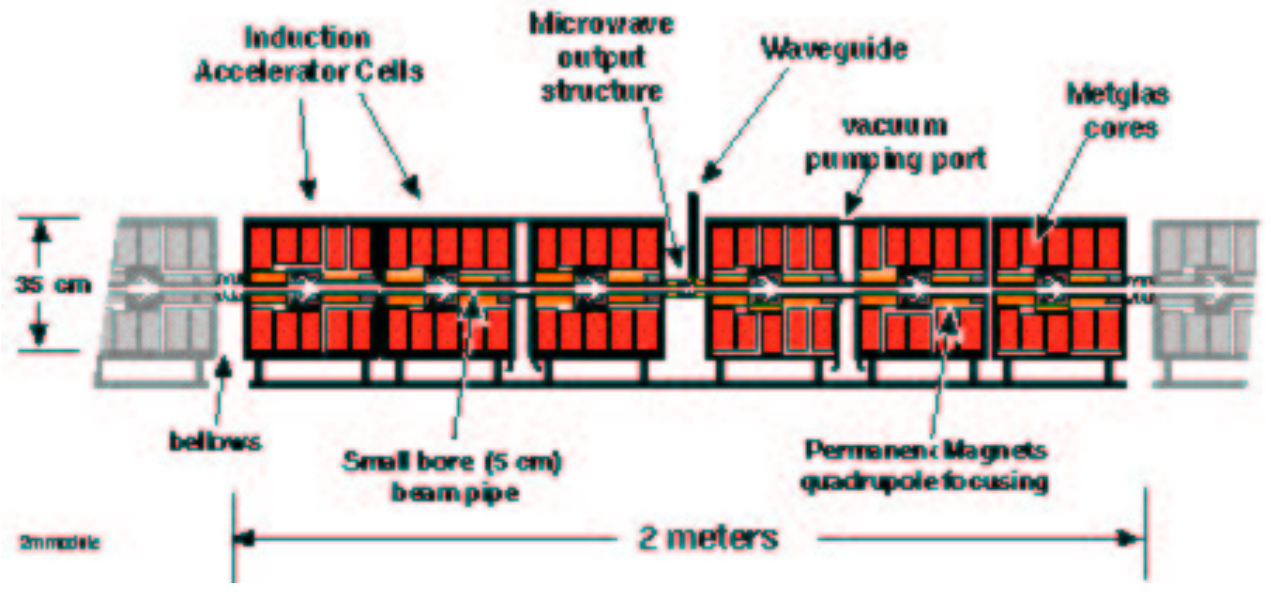

Figure 3.3: Schematic of a 2-m RK-TBA module.

generate "useful" rf power. But the fall portion is wasted. The corresponding loss in efficiency due to finite fall time is included when we consider the efficiency of the pulse power system.

\subsection{Main TBA}

The main TBA consists of identical 2-m modules. The key elements of a module are the permanent quadrupole magnets for focusing of the drive beam, the induction cells for reacceleration, and one rf extraction cavity. Design considerations for each of these major components is discussed below. The key issues in the design are of course related to the physics of rf extraction, reacceleration, and transport. However, much attention was paid to making the module compact and efficient. The resulting unit has a diameter of $35 \mathrm{~cm}$, which is quite small compared to existing induction accelerators. A schematic of the 2-m module is shown in Figure 3.3.

\subsubsection{Pipe size}

In the design of induction accelerators, the size of the beam tube is usually determined by $\mathrm{BBU}$ considerations, since the transverse impedance from the acceleration gap is inversely proportional to the square of the pipe radius. In our RK design, the low 
frequency BBU arising from the induction gaps is ameliorated by the low beam current of $600 \mathrm{~A}$ and Landau damping associated with the energy spread inherent in the rf buckets. With proper attention to the gap geometry, we were able to design a pipe with a radius of $2.5 \mathrm{~cm}$, and predict acceptable $\mathrm{BBU}$ growth.

\subsubsection{Permanent magnet focusing}

The small pipe radius allows us to take full advantage of permanent magnets, which have significant cost advantages when the focusing systems are small. Another major reason for the use of permanent magnets is the associated efficiency, as they eliminate the need for power supplies.

Several basic considerations govern the design of the FODO lattice. The foremost requirement is associated with a technique concocted to minimize the high frequency $\mathrm{BBU}$ growth arising from the $H E M_{11}$ mode in the rf extraction cavities. To suppress the instability growth, we demand that the betatron period be equal to the distance between adjacent extraction cavities $^{1}$, i.e. 2 meters. In addition, the phase advance per lattice period must be less than 90 degrees to ensure beam stability. Finally, the focusing strength must be sufficient to keep the beam envelope small enough to stay within the beam tube.

The zeroth order design equation is given by the thin lens approximation

$$
\sigma_{0}=\frac{\eta B L^{2}}{R[B \rho]}
$$

where $\sigma_{0}$ is the betatron phase advance per lattice period, and $\mathrm{B}$ is the quadrupole field strength at pole tip with radial position $\mathrm{R}$. $\mathrm{L}$ is the half lattice period, and $\eta$ the longitudinal occupancy factor for the quadrupole. The rigidity of the electron beam is given by

$$
[B \rho]=\frac{\beta \gamma m c}{e}
$$

where $\beta \gamma m c$ is the momentum of the relativistic beam. $[B \rho]$ has the value of 0.035 T-m for $10 \mathrm{MeV}$ electrons. A first order correction for deviations from the thin lens approximation is obtained by replacing the phase advance and occupancy factor with scaled variables given by [44]

$$
\widehat{\sigma_{0}}=\sqrt{2\left(1-\cos \sigma_{0}\right)}
$$

\footnotetext{
${ }^{1}$ This may also be achieved by placing the cavities at half this separation.
} 


$$
\widehat{\eta}=\eta \sqrt{2\left(1-\frac{2 \eta}{3}\right)}
$$

In our design, we $\sigma_{0}$ use of 60 degrees. There are 12 half-lattice-periods in 2 meters. The physical occupancy factor is 0.48 . The modified thin lens formula then gives a B-field at pole tip of 800 Gauss. This estimate is within a few \% of the actual B field required to give a $2-\mathrm{m}$ betatron period, when we include realistic spatial profile of the magnetic field, as well as the energy variations of the drive beam as it is accelerated over the 2 -m module.

A preliminary quadrupole design was constructed by Klaus Halbach [45] [46]. It consists of four rectangular blocks of ferrites with residual field $\mathrm{Br}$ of $3.2 \mathrm{kG}$. The dimensions of the blocks are $1.1 \mathrm{~cm} \times 3.22 \mathrm{~cm} \times 8 \mathrm{~cm}$. This design has as the first non-zero harmonic $n=10$, with $8.6 \%$ strength of the quadrupole field at aperture radius. Finite differential permeability effects have not been included in this preliminary design.

A basic requirement of the transport channel is that the focusing strength must be sufficiently strong to keep the beam from hitting the beam tube. The beam envelope in the focusing channel can be estimated from the equilibrium formula

$$
\frac{\varepsilon^{2}}{a^{2}}=\sigma_{0}^{2}\left(\frac{a}{L}\right)^{2}
$$

where $\varepsilon=\varepsilon_{n} / \beta \gamma$ and $a$ are respectively the unnormalized edge emittance and the edge radius of the beam envelope. With a normalized edge emittance of $600 \pi \mathrm{mm}$-mr, (which is achievable with a good gun design), we obtain an edge radius of about $2 \mathrm{~mm}$ for the beam.

\subsubsection{Steering and focusing corrections}

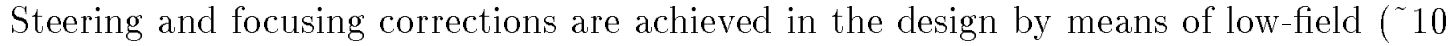
Gauss) dc coils located in the region immediately outside of the permanent magnets, at radial positions of about $4 \mathrm{~cm}$ from axis. These correction coils can be manufactured inexpensively on printed circuit boards. Since the beam apertures are small, particularly around the extraction cavities, beam centroid displacements must be kept to a minimum. Quadrupole misalignments, when combined with energy variations from head to tail, can lead to beam offsets that change over the length of the pulse, i.e. the 'corkscrew' phenomenon [47]. Our strategy for minimizing beam displacements and associated corkscrew is to impose misalignment tolerances that are not excessively tight, 
design for energy flatness of $1 \%$, and to correct alignment errors with closely spaced dipole steering coils. A key ingredient for the suppression of high frequency BBU is that the betatron period be equal to the distance between adjacent cavities. To ensure that this requirement is obeyed, a feedback system with 2 correction quadrupoles per betatron period is incorporated into the design. A possible monitor for the betatron motion is to use the rf output from the extraction cavities at the dipole frequency. Whether the signal sensitivity is adequate for this purpose needs further study.

\subsubsection{Induction Cores}

The design of induction cells for reacceleration is based on the relation

$$
V \cdot \Delta t=A \cdot F_{p} \cdot \Delta B
$$

where the core size (with magnetic flux over area $A$ and $F_{p}$ is the packing factor, or the fraction of the total core that is occupied by magnetic material) is determined by the required voltage $\mathrm{V}$ and pulse length $\Delta t$. The allowed flux swing $\Delta B$ is different for different magnetic material. Our design uses METGLAS ${ }^{2}$ with $\Delta B$ of $\sim 1.3$ T. Flux swings for METGLAS are several times larger than, for example, ferrites, which are commonly used in short pulse induction machines such as the Advanced Test Accelerator (ATA) [35] and the Experimental Test Accelerator II (ETA-II) [48]. The cores are correspondingly more compact. Furthermore, METGLAS is quite inexpensive, particularly when bought in large quantities. To determine the transverse dimensions of the core, (with outer radius $R_{o}$, and inner radius $R_{i}$ ), we recast (3.1) in terms of the accelerating gradient $\mathrm{G}$ and core occupancy factor $\eta_{c}$ (fraction of the axial length occupied by induction cores) as follows:

$$
R_{o}-R_{i}=G \Delta t /\left(\Delta B \eta_{c} F_{p}\right)
$$

where $F_{p}$ has a value of 0.65 to 0.75 for typical METGLAS cores. Since the accelerating gradient and pulse length are fixed by overall system requirements, our design philosophy was to maximize axial core occupancy. The proposed design has $\eta_{c}$ of 0.75 and core thickness $R_{o}-R_{i}$ of $10 \mathrm{~cm}$. Compact cores reduce cost as well as energy loss. Empirical measurements of core losses can be parameterized to give the following phenomenological formula for the core current (in Amps):

$$
I_{c}=360\left(R_{o}+R_{i}\right) \frac{\Delta B}{\Delta t}
$$

\footnotetext{
${ }^{2}$ METGLAS is a registered trademark of Allied-Signal, Inc.
} 


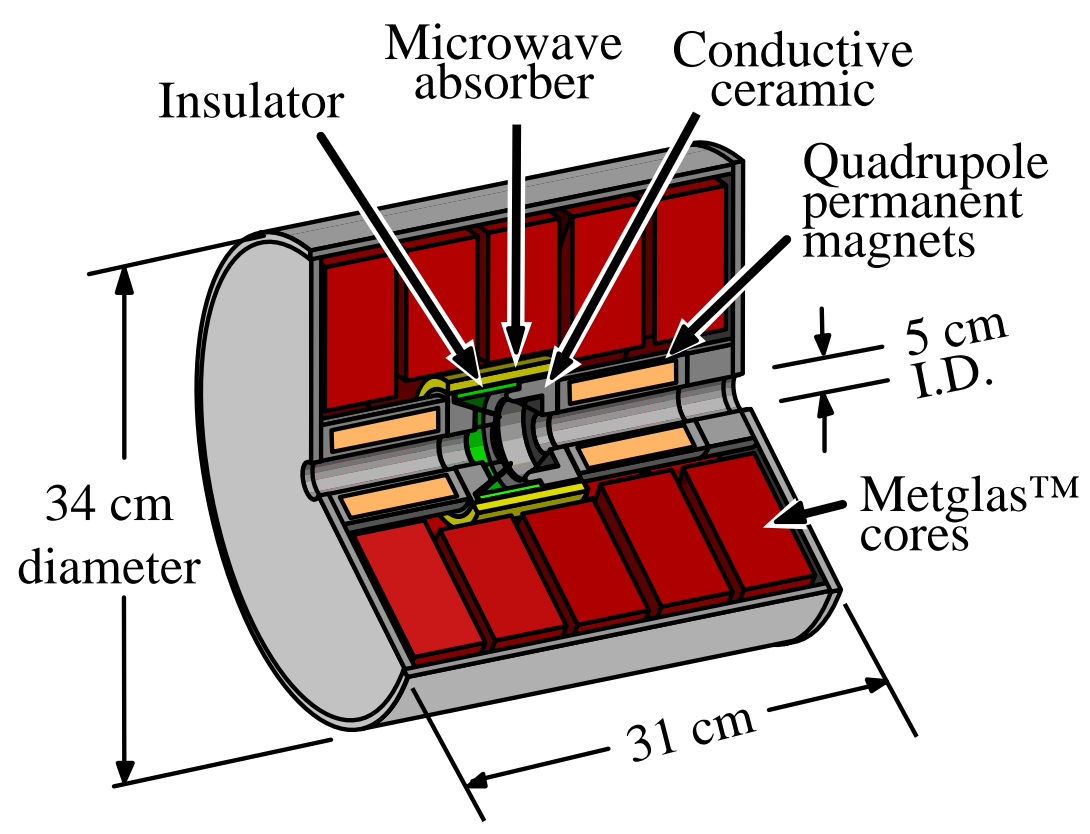

Figure 3.4: Proposed RK induction cell design.

where the radial dimensions are in meters, $\Delta B$ in Tesla, and $\Delta t$ in microseconds. The fraction of energy lost in the core $\left(I_{c} / I_{\text {total }}\right)$ is proportional to the size of the core. Our design parameters give a core current of about $117 \mathrm{~A}$. With a $600 \mathrm{~A}$ beam, the core efficiency is $80 \%$. The magnetic material in a $2 \mathrm{~m}$ module is packaged into 6 independent $100 \mathrm{kV}$ induction cells. Each cell in turn consists of five $20 \mathrm{kV}$ cores. Packaging into small induction cores provides a natural match to the low-voltage pulse power system. Figure 3.4 is an illustration of the proposed induction cell showing the five cores.

\subsubsection{Pulsed Power}

The desired voltage pulse from each core has a $100 \mathrm{~ns}$ linear rise, followed by a $200 \mathrm{~ns}$ flattop at $20 \mathrm{kV}$, followed by a fast fall. This voltage waveform is generated with pulseforming-networks (PFN's) with tapered impedance to match the induction core. Power input to the PFN's consists of a dc power supply and a Command Resonant Charging unit. The entire pulse power train is at low voltage, and no step-up transformers are needed. Hence, high efficiency and low cost is possible. 


\subsubsection{Acceleration Gap}

Each $100 \mathrm{kV}$ induction cell has an induction gap, which is one of the more critical components of the design. Ongoing design efforts evolve around the following key issues

- Reduction of high voltage breakdown risks - To have an induction gap that is safe from breakdown, the gradient across the insulator must be sufficiently low $(<$ $30 \mathrm{kV} / \mathrm{cm})$, sufficiently low fields on the metal surfaces $(<100 \mathrm{kV} / \mathrm{cm})$, proper shielding of the insulators from secondary electrons and $\mathrm{X}$-rays generated by the beam, and proper design of the triple-point (the interface between insulator, metal, and vacuum).

- Suppression of low frequency BBU - Dipole modes associated with the cavity (at a few $\mathrm{GHz}$ ) can lead to severe beam-break-up problems if not carefully damped. The required transverse impedance was achieved with heavy de-Q-ing by placing microwave absorbing material at critical locations around the cavity.

- Reduction of transverse and longitudinal impedances at high frequencies - While the high frequency BBU $\left(H E M_{11}\right.$ mode at $\left.{ }^{\sim} 14 \mathrm{GHz}\right)$ is generated primarily in the rf extraction cavities, and the major BBU suppression activities center around them, one must be careful to ensure that the transverse impedance contribution from the induction cavities are indeed negligible. In the present designs, the transverse impedance around $14 \mathrm{GHz}$ can be made to be lower than half an ohm, and its effect on the high frequency BBU is insignificant. The longitudinal impedance at $11.424 \mathrm{GHz}$ must be low to minimize the microwave power loss. Our design goal is to maintain the induction gap loss to less than $4 \%$, which requires that the longitudinal impedance be less than $2.4 \Omega$.

- Recent work on stacked insulators [49], composed of alternating layers of insulating material and conducting film, have shown impressive voltage hold-off capabilities factors of 1.5 to 4 times that of uniform dielectrics. Additionally, measurements of the rf properties of induction gaps utilizing these high gradient insulators (HGIs) [50] have demonstrated improvement by lowering the transverse and longitudinal impedances of concern for beam stability. 


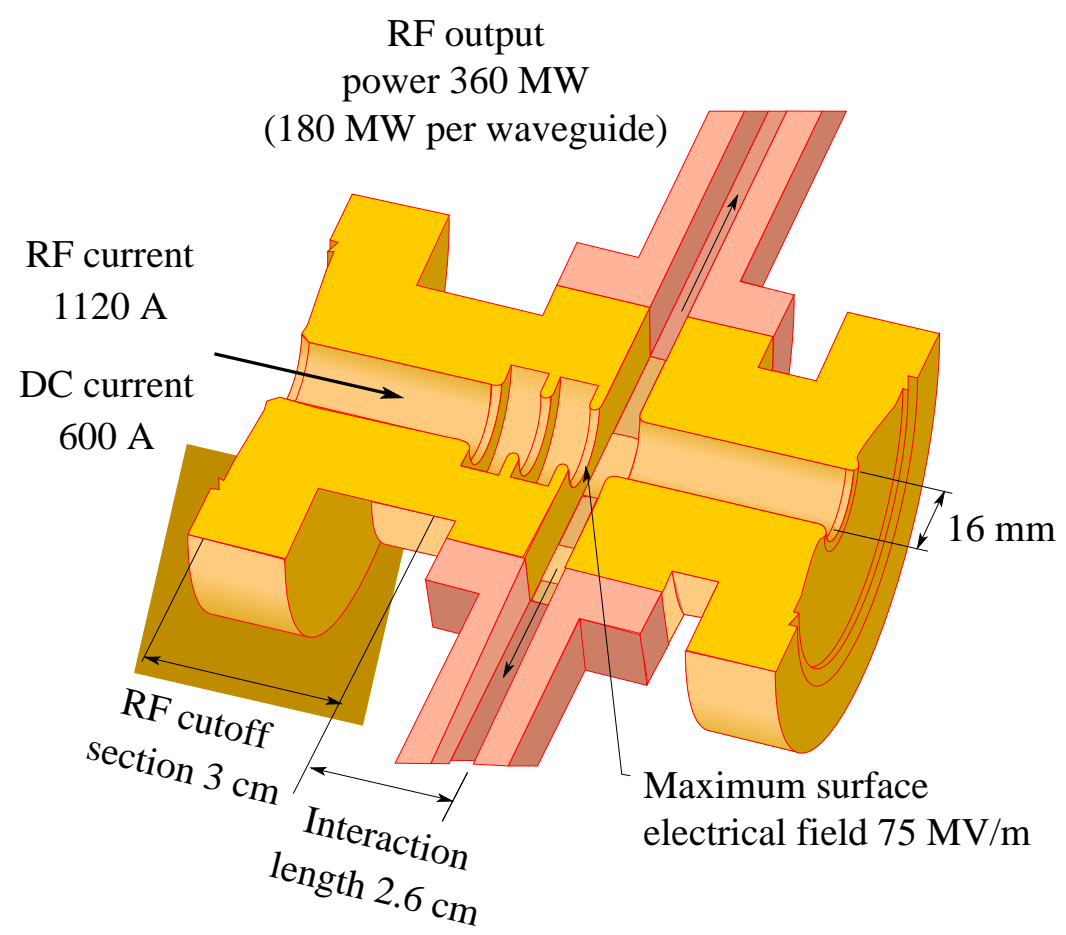

Figure 3.5: Illustration of an rf extraction structure.

\subsubsection{RF Extraction cavities}

Design of the rf extraction cavity is a major ongoing activity in theory and simulations. Present designs evolve around traveling-wave structures with 3 cells of 8 -mm inner radius 3 . The rf output is extracted through 2 separate ports in the $3 \mathrm{rd}$ cell, with $180 \mathrm{MW}$ each transported through separate waveguides, and fed directly into the two input couplers of the high gradient structure. An illustration of a proposed design is shown in Figure 3.5 .

Detail design of the cavities center around five key issues:

- Required output power - The extraction cavity must have the right effective impedance of about $540 \Omega$ in order to extract $360 \mathrm{MW}$ from a highly bunched beam (70 degree microbunch length) whose first harmonic current at $11.424 \mathrm{GHz}$ is about $1.15 \mathrm{kA}$.

- Minimal breakdown risks - Our present designs use traveling-wave-structures with 3 cells in order to reduce the surface fields. Surface field gradients of less than 70

\footnotetext{
${ }^{3}$ Other designs may use single-cell, standing wave out cavities
} 
$\mathrm{MV} / \mathrm{m}$ appear possible.

- Inductive detuning - In addition to generating the right amount of power, the rf cavities provide continuous longitudinal bunching for the drive beam. This is accomplished by inductively detuned traveling-wave-structures.

- Dipole de-Q-ing - The rf cavities must have low transverse impedances for the suppression of BBU. Fortunately, there is a natural de-Q-ing mechanism, as the required extraction cavities have inner radii of around $8 \mathrm{~mm}$, and the dipole modes in the cavity couple to the $T E_{11}$ mode in the pipe. Simulations to-date suggest that the resulting impedances are sufficiently low for BBU suppression provided that the 'Betatron Node Scheme' is used.

- Transverse focusing - The rf cavities have transverse fields associated with the fundamental mode. They can degrade the beam envelope if they are sufficiently strong and/or not properly corrected. Evaluation of these transverse forces from theory and simulations is ongoing.

\subsection{Front End and Back End}

Each RK unit has a front end that consists of an injector, a chopper, and an 'adiabatic compressor' section. At the end of each RK unit, there is an 'afterburner', followed by a final beam-dump. These five elements are briefly described below.

\subsubsection{Pre-chopper}

The injector is basically a short induction accelerator to generate an unbunched 300ns electron beam at $2.5 \mathrm{MeV}$. This section may consist of an induction injector (at 1 to $1.5 \mathrm{MeV}$ ), followed by a short induction accelerator section that takes the beam to $2.5 \mathrm{MeV}$. The required current from the injector is 1 to $1.5 \mathrm{kA}$, depending on the efficiency of the chopper which is required to produce at its exit a bunched beam with a dc current of 600 Amps. The current waveform required consists of a linear rise over 100 ns, followed by $200 \mathrm{~ns}$ of flat-top. The beam energy should be constant over the entire $300 \mathrm{~ns}$, including the front portion with the rising current. To generate the linearly rising current, the voltage pulse in the $1 \mathrm{MeV}$ injector will also, by the Child-Langmuir Law, have a (nonlinear) 100 ns rise time. The pulse-forming-network in the subsequent short accelerator section must be arranged to produce a higher voltage at the beam 
front, thereby compensating for the low energy at the injector exit. Since the electron beam will be transported over $300 \mathrm{~m}$ of narrow pipes, caution must be taken to minimize head-to-tail energy variations, as well as transverse beam offsets and beam temperature. The injector design should produce a bright beam with a normalized edge emittance of $600 \mathrm{~mm}-\mathrm{mr}$ or less., and transverse displacement of 200 microns or less.

\subsubsection{Chopper}

The purpose of the chopper is to generate cleanly separated microbunches at $11.4 \mathrm{GHz}$. The basic scheme follows the design of Haimson Research Corporation's Choppertron [51], a $11.4 \mathrm{GHz}$ microwave generator that has been deployed at LLNL. A subharmonic dipole deflecting cavity at $5.7 \mathrm{GHz}$ causes the electron beam to oscillate about a limiting aperture, leading to 'chopped' bunches at twice the oscillating frequency. A similar device can serve as the TBNLC front end chopper, except that much more effective heat dissipation is required for the $120 \mathrm{~Hz}$ operation of the NLC upgrade. Since a substantial amount of energy is lost on the chopper, there is incentive to make it more efficient. One idea is to precede the subharmonic deflecting cavity with conventional bunching cavities at $11.4 \mathrm{GHz}$. The role of the chopper then is primarily to 'clean up' the particles with the wrong phase. This requires straight-forward phasing of the $5.7 \mathrm{GHz}$ input power to the deflecting cavity relative to the incoming pre-bunched beam.

Other schemes are also possible. For lower current beams, it is not desirable to discard a significant portion of the current during the modulation process. A relativistic klystron input cavity and a series of gain cavities can then be utilized to produce the modulation with minimal loss of current, albeit at a lower energy. This scheme is discussed extensively in Chapter 12. For higher frequency applications, a different type of modulation process is needed - one that uses overmoded waveguides and travelling wave interactions. A higher energy beam is then needed in this case before modulation can be easily imposed. An experiment using a free electron laser interaction is described in Appendix B.

\subsubsection{Adiabatic Compressor}

The exiting beam from the chopper has microbunch lengths equivalent to 180 degrees or greater in longitudinal phase space. In the 'adiabatic compressor' region, the microbunch lengths are further reduced to 70 degrees which is the needed bunch length for longdistance propagation in the main TBA. This is accomplished by a number of idler cavities that are more inductive than the rf extraction cavities in the main TBA. In addition to 
microbunch sharpening, this section also serves to provide the energy transition from 2.5 $\mathrm{MeV}$ to $10 \mathrm{MeV}$. The induction core structures are very similar to the main RK. However, the voltage waveform should be flat over the $300 \mathrm{~ns}$ of beam-on time, so that the headto-tail energy flatness required for phase stability could be maintained at $10 \mathrm{MeV}$. The quadrupole magnets are weaker at the lower energies, and continues to increase with increasing energy, so that the betatron wavelength is kept fixed at $2 \mathrm{~m}$. Structurally, the 'adiabatic compressor' section looks very similar to the main TBA, except that no power is extracted from the idler rf cavities. However, power may be extracted from some or all of the idler cavities, provided that the induction modules compensate for the additional power loss. This may further enhance the net system efficiency.

\subsubsection{Afterburner}

At the end of the RK-TBA is an 'afterburner' section, the primary purpose of which is to increase overall system efficiency by extracting more power out of the nicely bunched beam at the end of the main TBA. This section has a number of rf extraction cavities, permanent magnets for focusing, but no reacceleration cells. The spacing of the rf cavities is changed as the average energy of the beam continues to decrease. The impedance of the cavities is also modified to compensate for changes in the rf bucket. The TBNLC design has 12 cavities in the 'afterburner' section, each generating $360 \mathrm{MW}$, and together covering a total of 12 meters.

\subsubsection{Beam-dump}

As the spent electron beam reaches the final beam dump, its average energy is less than $3 \mathrm{MeV}$. The design of this component is straightforward. 


\section{Part II}

\section{Methods of Simulation}



Tyger Tyger. burning bright,

In the forests of the night;

What immortal hand or eye,

Could frame thy fearful symmetry? In what distant deeps or skies.

Burnt the fire of thine eyes?

On what wings dare he aspire?

What the hand, dare sieze the fire?

And what shoulder, \& what art,

Could twist the sinews of thy heart?

And when thy heart began to beat,

What dread hand? \& what dread feet?

What the hammer? what the chain,

In what furnace was thy brain?

What the anvil? what dread grasp,

Dare its deadly terrors clasp:

When the stars threw down their spears

And water'd heaven with their tears:

Did he smile his work to see?

Did he who made the Lamb make thee?

Tyger Tyger burning bright,

In the forests of the night;

What immortal hand or eye,

Dare frame thy fearful symmetry?

- William Blake, The Tyger. 


\section{Chapter 4}

\section{Introduction}

Particle dynamics in relativistic klystrons pose several thorny problems for simulations. The electron beams are typically intense (100's to 1000's of Amperes) while only moderately relativistic (100's $\mathrm{keV}$ to 10 's of $\mathrm{MeV}$ ). Previous work [52] has shown that considerable microwave power can be developed in a relativistic klystron amplifier (RKA) configuration. With high beam current $(2-11 \mathrm{kA})$ and low kinetic energy ( $\sim 500 \mathrm{keV})$, these devices were typically limited by transport dynamics to single-outputs. The cavities comprising the rf circuit were designed to maximize the emitted microwave power at the output structure. Even though the peak output power was high, the total efficiency is still low, and much of the available energy in the beam was dumped. The concept of the relativistic klystron two-beam accelerator (RK-TBA) [2], [53] was developed to dramatically increase the device efficiency. In these devices, less charge is transported ( 0.5-2 kA), but at greater kinetic energy (1-10 MeV) than in an RKA. Also, the rf circuit is designed to produce only the required power for a single or, at most, double length of high gradient accelerating structure. As a result, the beams in RK-TBA's are much stiffer and are perturbed much less than in an RKA configuration. They may be transported over long distances to produce useful rf power in tens or hundreds of output structures. The use of suitably designed induction re-acceleration cavities and permanent quadrupole focusing increases the net efficiency of these devices to a level that is very competitive with traditional klystrons. The stiffness of the beams make them attractive candidates for producing rf power at higher frequencies than traditional klystrons can attain.

RK-TBA's are prone to several potentially debilitating beam instabilities. A lowimpedance, low-frequency transverse beam breakup (BBU) mode resides within the in- 
duction cavities, while a high-impedance, high-frequency BBU mode is present within the rf output structures. Increasing the beam's energy spread induces rapid betatron phase mixing which effectively cancels the effects of the low-frequency mode, and adjusting the focusing lattice to place the rf output structures at integral betatron wavelength separation can reduce the growth in transverse BBU from exponential to linear. This linear growth will eventually limit the length of the device, placing a cap on achievable efficiency. The induction cell cavities may also respond to the beam modulation and extract rf power and/or affect the bunching.

Possibly the most important element of the dynamics in an RK-TBA occurs in the longitudinal phase space. The beam is modulated at high frequencies (11-40 GHz), and each bunch carries a charge of 10's of nC. Space charge effects will produce debunching forces (a capacitive impedance) which is counteracted by (inductively) detuning the rf output structures. A bunch will undergo numerous synchrotron oscillations during transport through the full-scale device. Also, the bunches are not short compared to the rf wavelength; they typically subtend 60-120 degrees of rf phase. Hence, they sample very nonlinear fields in the rf output structures.

From this description we can identify the main problems present in a device simulation. The beams are sufficiently intense that space charge forces present more than a small perturbation. The beamline elements are necessarily spaced close together, and this requires treatment of overlapping, nonlinear fringe fields. Transverse focusing is strong so that a complete betatron oscillation occurs between rf output cavities. Transverse emittance, while low, is still sufficiently large that particles at the beam edge sample significant nonlinearities present in the beamline elements. The instantaneous energy spread is large ( $\sim 10-100 \%)$ to handle the low-frequency BBU, and to produce the bunching by rf rotation. The particle simulation, of necessity, must track many, many particles to provide adequate sampling of both the beam phase space and the fields experienced.

Fully electromagnetic and relativistic particle-in-cell simulations, rampant in the plasma physics community can and do simulate all these processes. Codes of this type $^{1}$ have found their way into the accelerator community as a means of studying high-perveance beams in klystrons, for example. However, the computational resources required are far too great to perform routine design and optimization on significant lengths of the full-scale TBA. On the other hand, typical tracking $\operatorname{codes}^{2}$ in the acceler-

\footnotetext{
${ }^{1}$ MAGIC, SOS, ARGUS, ISIS, MAFIA, etc.

${ }^{2}$ PARMELA, TRANSPORT, TURTLE, MAD, MARYLIE, COSY, etc. See the descriptions in the Los Alamos Accelerator Code Group (LAACG) internet web-site.
} 
ator physics community track a relatively small number of particles with a small phase space extent in complex external field environments. Self-field interactions are either completely neglected since the beams are usually tenuous and ultra-relativistic, or included only approximately with semi-analytic models. Maxwell-Vlasov, Fokker-Planck, and envelope codes can track beam distributions in the presence of self-fields, but these tend to track only a few of the lower order moments, while ignoring evolution of the detailed phase space distributions.

A simulation code development effort has been ongoing since the conception of the device scheme. The first RK codes [54] modeled the one-dimensional dynamics of the longitudinal phase space in a formalism similar to that used to model free-electron laser interactions [55]. These codes model the beam-cavity dynamics as a continuous interaction, and introduce self-field effects in an approximate and analytic manner. The creation and evolution of a stable bucket can be studied with these codes by tracking up to a few thousands of particles. This generation of codes was useful in examining the results of the initial series of RK experiments.

The next generation of codes [56] modeled time-dependent, two-dimensional ( $\mathrm{r}-\mathrm{z}$ ) beams and the beam-cavity interaction. These codes use the stationary cavity mode field distributions generated by finite-difference electromagnetic analysis codes (SUPERFISH, etc.), and advance the cavity rf fields via an equivalent circuit equation. Individual particles are pushed forward in time by integrating the Newton-Lorentz equations of motion. Self-field effects are again calculated with semi-analytic formulae, assuming azimuthal symmetry. Beamline magnetostatic elements with fringe fields can be simulated. Threedimensional beam distributions can be tracked, but significant transverse quadrupole moments will introduce errors into the self-field calculation. This makes it difficult to study transverse beam dynamics along a beamline with mixed symmetry components solenoid, monopole, dipole, quadrupole. Also, the rather low number of macroparticles prevents detailed statistical studies of beam distribution evolution. This generation of codes was used to analyze the MOK and Choppertron-II series of RK and RK-TBA experiments [57].

A parallel development effort has examined the problem of transverse dynamics in the beam transport line [58]. These codes are based on a slice-model of the longitudinal beam distribution. A circuit equation model of the interaction between the beam and the rf dipole modes is used to study cumulative BBU effects [59]. Detailed description of the beam distribution is missing, as are self-field effects and effects due to the conducting boundary. The monopole mode interaction with the induction and rf cavities is 
also absent or only roughly approximated. The results of these simulations showed the importance of a large ( $10 \%)$ bunch energy spread to alleviate the low-frequency BBU, as well as the utility of the 'betatron-node' scheme in reducing the growth rate of the high-frequency BBU.

There are three main components to this simulation. The first is a tracking algorithm to generate nonlinear transfer maps for pushing noninteracting particles through the external fields. A mapping algorithm is used so that tens or hundreds of thousands of macroparticles can be pushed from the solution of a few hundreds of differential equations. This is a great cost-savings device from the standpoint of CPU cycles. It can increase by several orders of magnitude the number of macroparticles that take place in the simulation, enabling more accurate modeling of the evolution of the beam distribution and enhanced sensitivity to effects due to the beam's halo. The second component in the code is a particle-in-cell (PIC) algorithm that solves a set of Helmholtz equations for the self-fields, including the conducting boundary condition, and generates impulses that are interleaved with the maps by means of a split-operator algorithm. The third component is an equivalent circuit equation solver that advances the modal rf cavity fields in time due to excitation by the modulated beam. Phase-space partitioning is realized by a multi-fiducial algorithm which can produce high accuracy with lowerorder dynamical calculations.

Chapter 5 derives the expansion of the Hamiltonian power series that we will need for linear and nonlinear beam dynamics calculations. This series is represents an expansion about a known orbit (the fiducial). In previous work, this fiducial orbit was assumed to lie along the beamline axis. The work presented here is original and extends the previous results by allowing for the fiducial trajectory to have arbitrary off-axis excursion, and to derive the Hamiltonian power series in this case.

Chapter 6 is a review of the Lie Algebraic perturbation techniques for constructing transfer maps for beam dynamics and tracking applications. This is mainly presented for completeness, but also as a short primer on these methods as they have been developed in the literature over the past 20 years.

Chapter 7 is an original and detailed derivation of the analytic, and semi-analytic, description of the fields present in the numerous beamline elements included in the simulation. The goal is to derive a power series expansion for the vector potential of magnetostatic, electrostatic, and electromagnetic elements. These expressions are derived from first principles from Maxwell's equations and from the underlying symmetry present in the element itself. These power series are then incorporated into the beamline 
Hamiltonian.

Chapter 8 is a description of the algorithm that calculates the mapping produced by the self-fields of the beam in the presence of conducting boundaries. The derivation of the impulse mapping and the potential formulation is original work. I have adapted a discrete Fourier analysis to convert a $3-\mathrm{D}$ potential problem into a set of 2-D problems. The 2-D problems find their solution with a multi-grid algorithm developed in the literature, but refined by me to account for conducting boundaries with non-conformal geometries.

Chapter 9 presents the theory and implementation of the single mode beam-cavity interaction. The derivation of the equivalent circuit equation is well covered in the available literature. What is new and significant here is the introduction of a description of the beam using a periodic Klimontovich distribution. This will allow us to calculate the average interaction of the rf mode with any 6 -D beam distribution, automatically accounting for slippage and beam loading effects by incorporating actual particle trajectories. This method only accounts for individual cavity modes, but is many times faster than an electromagnetic PIC algorithm. Finally, the circuit equation is separated into two distinct time scales, and a first order ordinary differential equation is derived for the slow scale time evolution. This is then solved analytically in the limit of short (slow) time scale intervals. Hence, by observing closely what happens to a single bunch during its traversal of a cavity, we may study the transient dynamics in the entire system without the need to track consecutive bunches. This is another time saving feature.

Chapter 10 presents the discussion of the multi-fiducial algorithm. This is an entirely original technique, and is found to have application in all other sectors of the code.

Finally, Chapter 11 presents a description of the architecture and capabilities of the simulation code that implements the preceding description of the physical environment and the dynamics involved in beam transport through an RK-TBA. However, the code is sufficently general in its application so that many other beam dynamics problems may be approached with the same framework. I present several tests of the code, to establish credibility and utility. 


\section{Chapter 5}

\section{The Model Hamiltonian}

\section{$5.1 \quad$ Introduction}

In this chapter we study the description of single particle motion derived from the classical relativistic Hamiltonian,

$$
H=q \Phi+\sqrt{\left(m c^{2}\right)^{2}+c^{2}(\vec{p}-q \vec{A})^{2}} .
$$

Here, the scalar and vector potentials depend only upon configuration space coordinates, and not upon the momenta. We will choose a gauge for the description of external fields that only produces a vector potential - the scalar potential is then only non-zero when self-fields are included. Hence, there is a natural division of the Hamiltonian description of the motion into sectors that depend upon only the configuration-space coordinates and those that depend upon the kinetic momenta coordinates. This becomes more evident in a latter section when we perform a power-series expansion upon the Hamiltonian and external vector potential.

For the beams of interest to us, with small velocity spreads, it is usually possible to obtain an accurate description of the self-field effects with an electrostatic approximation of the fields derived in the beam's rest frame. We will treat all effects of the beam's self-fields by first transforming to the rest frame. In that case, we neglect the self-field vector potential and consider only the scalar potential. In Chapter 8 , we will study the effects of this sector upon the beam dynamics. In Chapters 6 and 10 it will be shown how these collective effects can be calculated separately from single-particle effects, and the two sectors combined within a consistent, though approximate, Hamiltonian conceptual framework. 
We will thus neglect the action of the scalar potential and other collective effects in this chapter. Instead, we will use the Hamiltonian above to calculate single-particle orbits. The form of the Hamiltonian (5.1) above will be used to derive both exact equations of motion for a single particle in external fields (as defined by the external vector potential), and a power series expansion of the Hamiltonian (5.7), (5.8), (5.9) is then used as a basis for an efficient algorithm to construct a linear and nonlinear mapping which is used to propagate many, many particles at once. This is discussed further in Chapter 6.

\subsection{Derivation from the Action}

The action principle provides a theoretical framework to describe the canonical transformation needed to construct the Hamiltonian function we seek ${ }^{1}$. Hamilton's variational principle can be stated as a variation of the action:

$$
\delta \int d t L\left(\vec{q}, \frac{d \vec{q}}{d t}, t\right)=0
$$

where the configuration space variables are denoted by q. Assuming that a Hamiltonian function exists, this relation can be expressed as

$$
\delta \int d t L=\delta \int\left[\vec{p} \cdot \frac{d \vec{q}}{d t}-H(\vec{q}, \vec{p}, t)\right] d t=0
$$

where the variables, $\mathrm{p}$, are the usual canonical momenta.

\subsubsection{Poincaré-Cartan Form}

The Poincaré-Cartan form $d \omega=\vec{p} \cdot d \vec{q}-H d t$ is a differential 1-form living in the $(2 \mathrm{~N}+1)$-dimensional extended phase space of the $\mathrm{N}$-degree of freedom mechanical system. The orbits generated by Hamilton's equations are purely geometric objects in this extended phase space, and not dependent upon any particular parameterization. Hence, in this extended phase space, time can be treated on an equal footing with the rest of the canonical coordinates. We may project the extended phase space onto another phase space by taking one of the coordinates to be the 'affine' parameter for the orbits. For accelerator systems, the convenient parameter is the distance along the beamline, $z$.

\footnotetext{
${ }^{1}$ This discussion presupposes a familiarity with action principles, Lagrangian and Hamiltonian formulations of mechanical systems, the theory of canonical transformations, and differential forms. Refer to [60], [61], [62].
} 
The Poincaré-Cartan form can be expressed as

$$
d \omega=\vec{p}_{\perp} \cdot d \vec{q}_{\perp}+p_{z} d z+p_{t} d t
$$

where $p_{t}=-H$ is the momentum canonical to time. Provided that the clauses of the Implicit Function Theorem ${ }^{2}$ are validated, we can express time as a function of beamline position, $z$, and so

$$
d \omega=\left[\vec{p}_{\perp} \cdot \frac{d \vec{q} \perp}{d z}+p_{t} \frac{d t}{d z}-K\right] d z
$$

where the new Hamiltonian, $\mathrm{K}$, is defined as

$$
K\left(\vec{q}_{\perp}, \vec{p}_{\perp}, t, p_{t}, z\right)=-p_{z} .
$$

The relativistic Hamiltonian in this new parameterization is then given by

$$
K=-q A_{z}-\sqrt{\left(\frac{p_{t}}{c}\right)^{2}-(m c)^{2}-\left(\vec{p}_{\perp}-q \vec{A}_{\perp}\right)^{2}}
$$

\subsubsection{Renormalization Transformation}

A second transformation can be performed that render the dynamical variables into a more convenient form. The relativistic nature of the particle orbits introduces both momentum $(m c)$ and energy $\left(m c^{2}\right)$ scales. The potentials, momenta, and coordinates are scaled accordingly. The variation of the action is immune to the effects of scalar multiplication. Hence, we may scale the Poincaré-Cartan form without changing the resulting equations of motion. We define a new form as

$$
d \widetilde{\omega}=d \omega / m c=\frac{\vec{p}_{\perp}}{m c} \cdot \vec{q}_{\perp}+\frac{p_{t}}{m c^{2}} d(c t)-\frac{K}{m c}
$$

or

$$
d \widetilde{\omega}=\widetilde{p}_{\perp} \cdot d \vec{q}_{\perp}+(-\gamma) d s-\widetilde{K},
$$

where $\widetilde{K}=K / m c, \widetilde{p}_{\perp}=\vec{p}_{\perp} / m c,-\gamma=p_{t} / m c^{2}$, and $s=c t$. Additionally, the potentials are normalized via $\widetilde{\Phi}=q \Phi / m c^{2}$ and $\widetilde{A}=q \vec{A} / m c$. With this normalization the Hamiltonian function becomes

$$
\widetilde{K}=-\widetilde{A_{z}}-\sqrt{(-\gamma)^{2}-1-\left(\widetilde{p}_{\perp}-\widetilde{A}_{\perp}\right)^{2}}
$$

\footnotetext{
${ }^{2}$ In particular, that $\frac{\partial H}{\partial p_{z}}=\frac{d z}{d t}$ is always positive definite.
} 


\section{$5.3 \quad$ Fiducial Orbit}

A single-particle guiding orbit (the fiducial orbit) is calculated which usually represents the motion of the beam centroid or barycenter, and which usually happens to follow the beamline axis $^{3}$. In this case, symmetry is maximized and effects due to self-fields and transverse field non-linearities are neglected. However, any physically realizable orbit may be used as the fiducial. Given a set of initial conditions, the equations of motion derived from the Hamiltonian (5.3) are solved to obtain an exact orbit for the fiducial particle. This orbit is represented as a function of $z$, and has the scalar components

$$
\xi_{g}(z)=\left(x_{g}, \tilde{p}_{x g}, y_{g}, \tilde{p}_{y g}, s_{g},-\gamma_{g}\right)(z)
$$

The equations of motion for the fiducial orbit are presented here for reference:

$$
\begin{aligned}
& x_{g}^{\prime}=\frac{\partial \widetilde{K}_{g}}{\partial \widetilde{p}_{x g}} \quad=\quad \frac{\left[\widetilde{p}_{x g}-\widetilde{A}_{x}\left(\xi_{g}\right)\right]}{\left[\gamma_{g}^{2}-1-\left(\widetilde{p}_{\perp g}-\widetilde{A}_{\perp g}\right)^{2}\right]^{1 / 2}} \\
& \tilde{p}_{x g}^{\prime}=-\frac{\partial \widetilde{K}_{g}}{\partial \widetilde{x}_{g}} \quad=\quad \frac{\partial \widetilde{A}_{z}}{\partial x_{g}}+\frac{\left[\widetilde{p}_{x g}-\widetilde{A}_{x}\left(\xi_{g}\right)\right] \frac{\partial \widetilde{A}_{x}}{\partial x_{g}}}{\left[\gamma_{g}^{2}-1-\left(\widetilde{p}_{\perp g}-\widetilde{A}_{\perp g}\right)^{2}\right]^{1 / 2}}, \\
& y_{g}^{\prime}, \widetilde{p}_{y g}^{\prime} \text { similarly, } \\
& s_{g}^{\prime} \quad=\frac{\gamma_{g}}{\left[\gamma_{g}^{2}-1-\left(\tilde{p}_{\perp g}-\widetilde{A}_{\perp g}\right)^{2}\right]^{1 / 2}}, \\
& \left(-\gamma_{g}\right)^{\prime}=\frac{\partial \widetilde{A}_{z}}{\partial s_{g}}+\frac{\left[\widetilde{p}_{x g}-\widetilde{A}_{x}\left(\xi_{g}\right)\right] \frac{\partial \widetilde{A}_{x}}{\partial s_{g}}+\left[\widetilde{p}_{y g}-\widetilde{A}_{y}\left(\xi_{g}\right)\right] \frac{\partial \widetilde{A}_{y}}{\partial s_{g}}}{\left[\gamma_{g}^{2}-1-\left(\tilde{p}_{\perp g}-\widetilde{A}_{\perp g}\right)^{2}\right]^{1 / 2}}
\end{aligned}
$$

\subsection{Deviation Hamiltonian}

The fiducial orbit described above produces a particular 'chart' in phase space, connecting initial to final values. For particles with initial conditions near those of the fiducial particle, the chart is equivalent to a mapping, taking initial values to final values. This mapping can be derived analyically from the fiducial orbit and the underlying

\footnotetext{
${ }^{3}$ I say 'usually' here because that is the case of greatest frequency of usage. However, complicated beam dynamics may dictate another relation between fiducial and barycenter, misaligned optics may displace the beam axis from the optical axis, etc.
} 
Hamiltonian flow.

\subsubsection{Canonical Transformation to Deviation Coordinates}

Individual particles are characterized in their initial values by the difference of their phase space coordinates from that of the fiducial orbit. The phase space coordinates of a given particle are defined as

$$
\xi(z)=\left(x, \widetilde{p_{x}}, y, \widetilde{p_{y}}, s,-\gamma\right)(z)
$$

The deviation coordinates are defined as

$$
\widehat{\xi}(z)=\xi(z)-\xi_{g}(z)=\left(\widehat{x}, \delta_{x}, \widehat{y}, \delta_{y}, \widehat{s}, \delta_{s}\right)(z),
$$

where $\xi_{g}(z)$ denotes the coordinates of the fiducial, or 'guide', orbit. These fiducial coordinates are scalar functions of the affine parameter, $z$, and are used to construct a canonical transformation via the type- 2 generating function

$$
F_{2}\left(x, \delta_{x}, y, \delta_{y}, s, \delta_{s}, z\right)=\left(x-x_{g}\right)\left(\delta_{x}+\widetilde{p_{x g}}\right)+\left(y-y_{g}\right)\left(\delta_{y}+\widetilde{p_{y g}}\right)+\left(s-s_{g}\right)\left(\delta_{s}-\gamma_{g}\right) .
$$

The resulting Hamiltonian in the deviation coordinates is given by

$$
\widehat{K}\left(\widehat{x}, \delta_{x}, \widehat{y}, \delta_{y}, \widehat{s}, \delta_{s}\right)=\widetilde{K}+\frac{\partial F_{2}}{\partial z}
$$

Carrying out the derivation yields

$$
\begin{aligned}
\widehat{K} & =-\widetilde{A_{z}}\left(\widehat{r}_{\perp}+\overrightarrow{r_{g}}, \widehat{s}+s_{g}\right)-\sqrt{\left(\delta_{s}-\gamma_{g}\right)^{2}-1-\left(\widetilde{\delta_{\perp}}+\widetilde{p_{\perp g}}-\widetilde{A_{\perp}}\left(\widehat{r}_{\perp}+\overrightarrow{r_{g}}, \widehat{s}+s_{g}\right)\right)^{2}} \\
& +\left(x-x_{g}\right) \widetilde{p_{x g}}-x_{g}^{\prime}\left(\delta_{x}+\widetilde{p_{x g}}\right)+\left(y-y_{g}\right) \widetilde{p_{y g}}-y_{g}^{\prime}\left(\delta_{y}+\widetilde{p_{y g}}\right)+\left(s-s_{g}\right)\left(-\gamma_{g}\right)^{\prime}-s_{g}^{\prime}\left(\delta_{s}-\gamma_{g}\right) .
\end{aligned}
$$

\subsubsection{Power Series Expansion}

This Hamiltonian can now be expanded in a power series ${ }^{4}$ in the deviation coordinates,

$$
\widehat{K}=\widehat{K_{0}}+\widehat{K_{1}}+\widehat{K_{2}}+\widehat{K_{3}}+\widehat{K_{4}}+\cdots
$$

\footnotetext{
${ }^{4}$ This power series thus contains many expansion coefficients for a six-dimensional phase space, not all of which are independent from each other. Use of a Lie algebraic representation instead of a Taylor series representation, reduces the number of coefficients to the minimum required at any order, while still maintaining (at least approximately) the constraint of symplecticity.
} 
The lowest-order term in this expansion does not contribute to the equations of motion since it is only a function of $z$, and so is neglected. By defining the particle phase space coordinates in terms of deviations from the fiducial orbit coordinates, the linear term in the expansion is explicitly zero ${ }^{5}$. The remaining terms are of second- and higher-order in the deviation coordinates. For small deviations from the fiducial orbit, this power series will rapidly converge. We will only consider terms in the Hamiltonian up to fourth-order. This gives equations of motion valid to third order.

It is useful to perform this power series expansion to examine the effects of particular nonlinearities. We first introduce a little shorthand notation. The vector potential is also represented as a power series expansion in the configuration space coordinates about the fiducial orbit

$$
\begin{aligned}
\widetilde{A_{z}} & =\widetilde{A_{z 0}}+\widetilde{A_{z 1}}+\widetilde{A_{z 2}}+\widetilde{A_{z 3}}+\widetilde{A_{z 4}}+\cdots \\
\widetilde{A_{\perp}} & =\widetilde{A_{0}}+\widetilde{A_{1}}+\widetilde{A_{2}}+\widetilde{A_{3}}+\widetilde{A_{4}}+\cdots \\
& =\widetilde{A_{0}}+\widetilde{A_{+}}
\end{aligned}
$$

The lowest-order term in this expansion give the values of the vector potential components along the fiducial orbit, with higher-order terms adding contributions due to deviations from this orbit.

The fiducial orbit relativistic factor, $\gamma_{g}$, has an associated normalized velocity, $\beta_{g}=$ $\sqrt{1-1 / \gamma_{g}^{2}}$. Of course, this assumes that the fiducial orbit does not stop propagating forward and reverse direction. If this were to occur, the clauses of the Implicit Function Theorem would be violated and the entire framework presented here would be invalid. This could conceivably be tolerated as long as the calculation of the maps ceases when the forward momentum of the fiducial reaches zero. At this point, we could switch Hamiltonians from $K=-p_{z}$ to $H=-p_{t}$, re-parameterizing the phase space, and keep integrating the equations of motion. Physically, we are only in danger of invalidation if we were to attempt modeling systems like conventional klystrons, where the beam is violently decelerated over a short distance. Fortunately, that is not the case in RK-TBA systems.

The kinetic momentum differs from the canonical momentum which appears in the Hamiltonian functions. If we denote the (normalized) transverse kinetic momentum by

\footnotetext{
${ }^{5}$ In cases where the beamline axis does not coincide with the individual magnet axes, a linear term in the Hamiltonian is used to express the alignment error [63].
} 
the symbol $\tilde{\pi}$, where $\tilde{\pi}=\widetilde{p_{\perp}}-\widetilde{A_{\perp}}$, then we may write

$$
\begin{aligned}
& \widetilde{\pi_{0}}=\widetilde{p_{\perp g}}-\widetilde{A_{0}}=\gamma_{g} \overrightarrow{\beta_{\perp g}}, \\
& \widetilde{\pi_{1}}=\widetilde{\delta_{\perp}}-\widetilde{A_{1}} .
\end{aligned}
$$

Higher-order terms, $\widetilde{\pi_{2}}$, etc. can be defined as the opposites of the corresponding term in the transverse vector potential expansion, but this distinction will not be emphasized.

The deviation Hamiltonian is then expressible in the deviation variables as

$$
\begin{aligned}
\widehat{K} & =-\widetilde{A_{z}}-\sqrt{\left(\gamma_{g} \beta_{g}\right)^{2}-2 \gamma_{g} \delta_{s}+\delta_{s}^{2}-\left(\overrightarrow{\delta_{\perp}}+\widetilde{p_{\perp g}}-\widetilde{A_{\perp}}\right)^{2}}+\frac{\partial F_{2}}{\partial z} \\
& =-\widetilde{A_{z}}-\sqrt{\left(\gamma_{g} \beta_{g}\right)^{2}-\left(\widetilde{\pi_{0}}\right)^{2}-2 \gamma_{g} \delta_{s}+\delta_{s}^{2}-\delta_{\perp}^{2}-2 \overrightarrow{\delta_{\perp}} \cdot\left(\widetilde{\pi_{0}}-\widetilde{A_{+}}\right)-2 \widetilde{p_{\perp}} \cdot \widetilde{A_{+}}-2 \widetilde{A_{0}} \cdot \widetilde{A_{+}}-\left(\widetilde{A_{+}}\right)^{2}} \\
& +\frac{\partial F_{2}}{\partial z} .
\end{aligned}
$$

The total energy of a particle is given by the original Hamiltonian (5.1). In the renormalized variables we then see that

$$
\gamma^{2}=\frac{H^{2}}{\left(m c^{2}\right)^{2}}=(1+\widehat{T})^{2}=1+(\tilde{p}-\widetilde{A})^{2}
$$

where $(\widehat{T})$ is the normalized kinetic energy, and

$$
(\widetilde{p}-\widetilde{A})^{2}=\gamma^{2}-1=(\gamma \vec{\beta})^{2}=\left(\gamma \beta_{z}\right)^{2}+\left(\gamma \overrightarrow{\beta_{\perp}}\right)^{2}
$$

The total kinetic energy may be split into longitudinal and transverse parts,

$$
\begin{aligned}
(\tilde{p}-\tilde{A})^{2} & =\left(\widetilde{p_{\perp}}-\widetilde{A}_{\perp}\right)^{2}+\left(\widetilde{p_{z}}-\widetilde{A_{z}}\right)^{2}, \\
\left(\widetilde{p_{z}}-\widetilde{A_{z}}\right)^{2} & =\left(\gamma \beta_{z}\right)^{2}, \\
\left(\widetilde{p_{\perp}}-\widetilde{A}_{\perp}\right)^{2} & =\left(\gamma \overrightarrow{\beta_{\perp}}\right)^{2} .
\end{aligned}
$$

In the case of the fiducial orbit, we see that the transverse kinetic energy as defined above is

$$
\left(\widetilde{\pi_{g}}\right)^{2}=\left(\widetilde{p_{\perp g}}-\widetilde{A_{0}}\right)^{2}=\left(\gamma_{g} \overrightarrow{\beta_{\perp g}}\right)^{2}=\left(\gamma_{g} \overrightarrow{\beta_{g}}\right)^{2}-\left(\gamma_{g} \beta_{z g}\right)^{2} .
$$

Combining terms in the deviation Hamiltonian

$$
\widehat{K}=-\widetilde{A_{z}}-\left\{\left(\gamma_{g} \beta_{z g}\right)^{2}-2 \gamma_{g} \delta_{s}+\delta_{s}^{2}-\delta_{\perp}^{2}-2 \overrightarrow{\delta_{\perp}} \cdot \widetilde{\pi_{0}}+2 \widetilde{\pi_{0}} \cdot \widetilde{A_{+}}-\left(\widetilde{A_{+}}\right)^{2}\right\}^{1 / 2}+\frac{\partial F_{2}}{\partial z}
$$


After a little algebra, we obtain

$$
\widehat{K}=-\widetilde{A_{z}}-\left(\gamma_{g} \beta_{z g}\right)\left\{1-\frac{1}{\left(\gamma_{g} \beta_{z g}\right)^{2}}\left[\delta_{\perp}^{2}-\delta_{s}^{2}+2 \gamma_{g} \delta_{s}+2 \widetilde{\pi_{0}} \cdot\left(\overrightarrow{\delta_{\perp}}-\widetilde{A_{+}}\right)+{\widetilde{A_{+}}}^{2}\right]\right\}^{1 / 2}+\frac{\partial F_{2}}{\partial z}
$$

This expression is now in a state from which we can derive terms of the power series representation. We can see that the terms of the series represent powers of the ratio of [momentum deviation / forward momentum]. Thus, this series will converge more rapidly for colder/more relativistic beams, and become less accurate at a given order of truncation for hotter/less relativistic beams. It should be noted, however, that the ratio is what really matters. The fiducial trajectory is, in theory, calculated exactly. It may represent an orbit with classical or relativistic energies equally well (as long as it doesn't reflect at some position $z$ ).

Performing the power series expansion on (5.6) to fourth-order, neglecting terms which are independent of the deviation coordinates, and noting that terms first order in $\vec{\xi}$ cancel identically with the last term, we find (dropping the 'g' subscript from guide orbit quantities, $\gamma_{g}, \beta_{z g}$ ) after some extremely tedious algebra

$$
\begin{aligned}
& \widehat{K_{2}}=-\widetilde{A_{z 2}}-\frac{5\left({\widetilde{\pi_{0}}}^{2}\right)^{3}+6\left(\gamma \beta_{z}\right)^{2}\left({\widetilde{\pi_{0}}}^{2}\right)^{2}+8\left(\gamma \beta_{z}\right)^{4}\left({\widetilde{\pi_{0}}}^{2}\right)+16\left(\gamma \beta_{z}\right)^{6}}{32\left(\gamma \beta_{z}\right)^{7}}\left[\delta_{s}^{2}-{\widetilde{\pi_{1}}}^{2}+2 \widetilde{\pi_{0}} \cdot \widetilde{A_{2}}\right] \\
& +\frac{15\left({\widetilde{\pi_{0}}}^{2}\right)^{2}+12\left(\gamma \beta_{z}\right)^{2}\left({\widetilde{\pi_{0}}}^{2}\right)+8\left(\gamma \beta_{z}\right)^{4}}{16\left(\gamma \beta_{z}\right)^{7}}\left[\gamma \delta_{s}+\widetilde{\pi_{0}} \cdot \widetilde{\pi_{1}}\right]^{2}, \\
& \widehat{K_{3}}=-\widetilde{A_{z 3}}-\frac{5\left({\widetilde{\pi_{0}}}^{2}\right)^{3}+6\left(\gamma \beta_{z}\right)^{2}\left({\widetilde{\pi_{0}}}^{2}\right)^{2}+4\left(\gamma \beta_{z}\right)^{4}\left({\widetilde{\pi_{0}}}^{2}\right)+16\left(\gamma \beta_{z}\right)^{6}}{16\left(\gamma \beta_{z}\right)^{7}}\left[\widetilde{\pi_{0}} \cdot \widetilde{A_{3}}+\widetilde{\pi_{1}} \cdot \widetilde{A_{2}}\right] \\
& -\frac{15\left({\widetilde{\pi_{0}}}^{2}\right)^{2}+12\left(\gamma \beta_{z}\right)^{2}\left({\widetilde{\pi_{0}}}^{2}\right)+8\left(\gamma \beta_{z}\right)^{4}}{16\left(\gamma \beta_{z}\right)^{7}}\left[\gamma \delta_{s}+\widetilde{\pi_{0}} \cdot \widetilde{\pi_{1}}\right]\left[\delta_{s}^{2}-{\widetilde{\pi_{1}}}^{2}+2 \widetilde{\pi_{0}} \cdot \widetilde{A_{2}}\right] \\
& +\frac{5\left({\widetilde{\pi_{0}}}^{2}\right)+2\left(\gamma \beta_{z}\right)^{2}}{4\left(\gamma \beta_{z}\right)^{7}}\left[\gamma \delta_{s}+\widetilde{\pi_{0}} \cdot{\widetilde{\pi_{1}}}^{3}\right]^{3} \\
& \widehat{K_{4}}=-\widetilde{A_{z 4}}-\frac{5\left({\widetilde{\pi_{0}}}^{2}\right)^{3}+6\left(\gamma \beta_{z}\right)^{2}\left({\widetilde{\pi_{0}}}^{2}\right)^{2}+8\left(\gamma \beta_{z}\right)^{4}\left({\widetilde{\pi_{0}}}^{2}\right)+16\left(\gamma \beta_{z}\right)^{6}}{32\left(\gamma \beta_{z}\right)^{7}}\left[-{\widetilde{A_{2}}}^{2}+2 \widetilde{\pi_{1}} \cdot \widetilde{A_{3}}+2 \widetilde{\pi_{0}} \cdot \widetilde{A_{4}}\right] \\
& -\frac{15\left({\widetilde{\pi_{0}}}^{2}\right)^{2}+12\left(\gamma \beta_{z}\right)^{2}\left({\widetilde{\pi_{0}}}^{2}\right)+8\left(\gamma \beta_{z}\right)^{4}}{8\left(\gamma \beta_{z}\right)^{7}}\left[\gamma \delta_{s}+\widetilde{\pi_{0}} \cdot \widetilde{\pi_{1}}\right]\left[\widetilde{\pi_{0}} \cdot \widetilde{A_{3}}+\widetilde{\pi_{1}} \cdot \widetilde{A_{2}}\right] \\
& +\frac{15\left({\widetilde{\pi_{0}}}^{2}\right)^{2}+12\left(\gamma \beta_{z}\right)^{2}\left({\widetilde{\pi_{0}}}^{2}\right)+8\left(\gamma \beta_{z}\right)^{4}}{64\left(\gamma \beta_{z}\right)^{7}}\left[\delta_{s}^{2}-{\widetilde{\pi_{1}}}^{2}+2 \widetilde{\pi_{0}} \cdot \widetilde{A_{2}}\right]^{2} \\
& -\frac{15\left({\widetilde{\pi_{0}}}^{2}\right)+6\left(\gamma \beta_{z}\right)^{2}}{8\left(\gamma \beta_{z}\right)^{7}}\left[\gamma \delta_{s}+\widetilde{\pi_{0}} \cdot{\widetilde{\pi_{1}}}^{2}\left[\delta_{s}^{2}-{\widetilde{\pi_{1}}}^{2}+2 \widetilde{\pi_{0}} \cdot \widetilde{A_{2}}\right]\right.
\end{aligned}
$$




$$
+\frac{5}{8\left(\gamma \beta_{z}\right)^{7}}\left[\gamma \delta_{s}+\widetilde{\pi_{0}} \cdot \widetilde{\pi_{1}}\right]^{4}
$$

\section{On-axis fiducial orbit}

In the situation where no dipole fields exist, and the fiducial orbit has no transverse excursion from the beamline axis, these expressions simplify immensely. In this case, we see that $\widetilde{\pi_{0}}=0=\vec{\beta}_{\perp}$, and $\beta_{z}=\beta=\sqrt{1-1 / \gamma^{2}}$. The deviation Hamiltonian series $(5.7,5.8,5.9)$ takes the limiting form,

$$
\begin{gathered}
\widehat{K_{2}}=-\widetilde{A_{z 2}}-\frac{1}{2(\gamma \beta)}\left[\delta_{s}^{2}-{\widetilde{\pi_{1}}}^{2}\right]+\frac{1}{2(\gamma \beta)^{3}}\left[\gamma \delta_{s}\right]^{2} \\
\widehat{K_{3}}=-\widetilde{A_{z 3}}-\frac{1}{(\gamma \beta)}\left[\widetilde{\pi_{1}} \cdot \widetilde{A_{2}}\right]-\frac{1}{2(\gamma \beta)^{3}}\left[\gamma \delta_{s}\right]\left[\delta_{s}^{2}-{\widetilde{\pi_{1}}}^{2}\right]+\frac{1}{2(\gamma \beta)^{5}}\left[\gamma \delta_{s}\right]^{3} \\
\widehat{K_{4}}=-\widetilde{A_{z 4}}-\frac{1}{2(\gamma \beta)}\left[-{\widetilde{A_{2}}}^{2}+2 \widetilde{\pi_{1}} \cdot \widetilde{A_{3}}\right]-\frac{1}{(\gamma \beta)^{3}}\left[\gamma \delta_{s}\right]\left[\widetilde{\pi_{1}} \cdot \widetilde{A_{2}}\right] \\
+\frac{1}{8(\gamma \beta)^{3}}\left[\delta_{s}^{2}-{\widetilde{\pi_{1}}}^{2}\right]^{2}-\frac{3}{4(\gamma \beta)^{5}}\left[\gamma \delta_{s}\right]^{2}\left[\delta_{s}^{2}-\widetilde{\pi}^{2}\right]+\frac{5}{8(\gamma \beta)^{7}}\left[\gamma \delta_{s}\right]^{4}
\end{gathered}
$$

These expansions are the main results of this chapter. Along with the equations of motion for the fiducial trajectory (5.4), the power series expansion (5.7), (5.8), (5.9) provides a basis for linear and order-by-order nonlinear analysis and solution of the equations of motion for the entire ensemble of particles comprising the beam. Comparing the two sets of expansions, $(5.7,5.8,5.9)$ and $(5.10)$, we see that the coupling of the off-axis fiducial momentum components with the vector potential introduces new terms. These can be treated and analyzed as pseudo-multipoles. In most cases of interest, the fields in the accelerator beamline are only weakly nonlinear, so that the power series can be seen to rapidly converge. Even in the cases (such as rf fields) where there is one coordinate (in the rf cavity case, the arrival time $\widehat{s}$ ) along which the fields have a strongly nonlinear variation, taking multiple fiducial elements along the range of that coordinate can restore the accuracy of the finite power series expansion. This is discussed further in Chapter 10.

\subsection{Computational Program}

The program for calculating the orbits is now clear from a conceptual standpoint. A given beamline interval is characterized by a vector potential that represents the external 
fields present. A beam is described by an ensemble of particles with individual initial coordinates at the entrance to the interval, and our goal is to obtain the final coordinates in the 6-D phase space for each particle, consistent with its traversing the interval.

A fiducial (or set of fiducials) is chosen and its initial coordinates determined. The set of equations (5.4) is then solved (either analytically or numerically) to obtain the final coordinates. This is an exact solution, in so much as the exact equations of motion are used in the determination of the fiducial orbit.

The larger task then remains to solve for the trajectories of every other particle in the beam ensemble. One method would be to solve (5.4) for each particle to obtain the exact solution. However, this is much too computationally intensive for an ensemble population that is large enough to describe the 6 -D phase space distribution of the beam in sufficient detail. Typically, this means that we will be using $\sim 10,000$ to $\sim 100,000$ or more particles. Additionally, we are free to choose which fiducials orbits to use in the initial calculation. By a judicious choice, we can guarantee that all other particles in the ensemble lie 'close' to these fiducials in the phase space of initial coordinates. In this way, we may consider an approximation scheme that distinguishes the linear beam dynamics and the various non-linear orders. What we seek then is a mapping algorithm that derives from the deviation Hamiltonian (5.6) or it's expansion (5.7), (5.8), (5.9), and a given fiducial orbit that may then be used to advance particles across the interval. This mapping will be calculated once at every interval for each fiducial. It will then be applied to each particle with coordinates near the given fiducial. Since this mapping is derived from a Hamlitonian, we may use the underlying symplectic structure to construct an algorithm for the efficient construction of maps. The techniques to do this are discussed in the next chapter. 


\section{Chapter 6}

\section{Lie Algebraic Formalism}

\subsection{Introduction}

In the last chapter, we described the formal procedure for constructing the relativistic Hamiltonian for a fiducial particle orbit and finding the equations of motion. Assuming we had a solution to that orbit, we constructed a power series of 'deviation' Hamiltonians with progressively higher degrees of nonlinearity. In this section, we'll describe a method of generating equations of motion, and solving them, in such a way as to construct a transfer map. A transfer map is an object that acts upon the phase space coordinates of a particle at an initial location along the beamline, and produces another set of coordinates at a final location along the beamline. The map is an analytic (and, in general, nonlinear) function of the initial phase space coordinates. This represents a method of performing arbitrary order 'paraxial' optics calculations. In the method presented here, we no longer care if the particles are close to the geometric axis of the beamline system. Rather, we are only concerned with deviations of the phase space coordinates of an arbitrary particle from the coordinates of the fiducial particle's orbit. The fiducial orbit was previously calculated to arbitrary degree of accuracy, and is allowed any excursion from the beamline axis. The maps we produce are equivalent to solving the equations of motion.

Prior to the development of Lie transformation methods, most transfer maps were based on a Taylor series approach [64]. For an $m$ degree of freedom system, the set of final coordinates, $\zeta^{f}$, can be related to the initial coordinates, $\zeta^{i}$, by

$$
\zeta_{a}^{f}=\sum_{b=1}^{2 m} R_{a b} \zeta_{b}^{i}+\sum_{1 \leq b \leq c}^{2 m} T_{a b c} \zeta_{b}^{i} \zeta_{c}^{i}+\sum_{1 \leq b \leq c \leq d}^{2 m} U_{a b c d} \zeta_{b}^{i} \zeta_{c}^{i} \zeta_{d}^{i}+\cdots
$$


(We have ignored constant terms in the expansion.) The equations of motion, a la Newton-Lorentz and Maxwell, can be written down and the coefficients in the above series determined. The expansion series coefficients can be (and have been) analyzed extensively. The first term contains all the linear dynamics, while nonlinear effects are contained in the subsequent terms.

This expansion is useful, but has two serious problems. Both pertain to symplecticity of the mapping when the equations of motion are derived from a Hamiltonian. First of all, not all the coefficients are independent (even after accounting for the obvious symmetry of index interchange), and we are doing too much work in calculating them all. Rather, they are linked by symplecticity - a nonlinear constraint. This point has been discussed by Wollnik [65] [66], who derives relations that link elements of the secondand third-order transfer matrices via the symplectic condition. Next, by truncating the series at some finite order, the map based on this series is no longer symplectic, and the map no longer faithfully represents a solution to the equations of motion generated by the Hamiltonian. For single-pass beamlines (like those considered here, as well as electron microscopes, etc.) the loss of strict symplecticity is not a grave issue. For storage rings, however, it can be a disaster.

The development of Lie tranformation techniques provided a systematic way of computing ray traces in linear and higher-order beam optics problems. The Lie algebraic treatment maintains the invariance of the fundamental Poisson brackets - and hence symplecticity - with a computationally economic algorithm. In fact, a Lie transformation represents the minimal amount of information required to construct a symplectic mapping at any given order of nonlinearity. This is what makes these techniques useful for our purposes.

This chapter borrows heavily from the published work of Dragt and his co-workers [63] [67] (and references therein), who performed most of the initial and subsequent development of the techniques described here. I will defer the formal presentation of the material to the cited references. Here, I merely wish to lay out the tools we use in constructing transfer maps, with a brief discussion where necessary.

\subsection{Hamiltonian Flows and Symplectic Maps}

We consider a dynamical system of $m$ degrees of freedom. We will only treat the case of an autonomous system ${ }^{1}$. In this case, there is a single parameter, usually the elapsed time

\footnotetext{
${ }^{1}$ In the case of a non-autonomous system, we may enlarge the dimension of the the system and consider the corresponding extended phase space, which in its newly parameterized form can be treated
} 
$t$, which can be used to characterize the state of the system. There exist $m$ independent coordinates defined on an $m$-dimensional configuration vector space or manifold ${ }^{2}$. These coordinates are denoted by $\vec{q}=\left\{q_{1}, q_{2}, \ldots, q_{m}\right\}$, and are scalar functions of $t$. Finding this set of scalar functions constitute what is meant by calculating the trajectory of the system in the configuration space.

In Hamiltonian dynamics, we define an associated canonical momentum, $p_{i}$, to each coordinate, $q_{i}$. The $2 m$-dimensional vector space, a differentiable manifold, with local coordinates given by

$$
\zeta=\left\{q_{1}, q_{2}, \ldots, q_{m}, p_{1}, p_{2}, \ldots, p_{m}\right\}
$$

is called the phase space of the dynamical system. Including the time, $t$, as another independent coordinate, defines a $2 m+1$-dimensional state space. A dynamical system on this space can be generated by a single scalar function of the phase space coordinates and the time, $H(\zeta, t)$, the Hamiltonian. Hamilton's equations are applied to this function to generate equations of motion in the state space. Solving the equations of motion finds a trajectory in the state space, subject to initial conditions. The set of trajectories generated by all possible initial conditions and the equations of motion defines the Hamiltonian flow. This flow is a vector field defined on a manifold, which has no a priori connection to any set of underlying coordinate bases. The flow is a purely geometric object. In a different sense, the flow defines the action of the Hamiltonian dynamics which connects intial states to final state. In this sense, the Hamiltonian flow defines a mapping, $\phi$. This mapping is generally a nonlinear function of the state-space coordinates. We use the notation

$$
\zeta^{f}=\phi \zeta^{i}
$$

to describe the action of the mapping upon the initial coordinates to generate the final coordinates. This mapping is the transfer map. It can be shown [69] that mappings derived from scalar functions on phase space are symplectic maps. Hence, every Hamiltonian generates a symplectic map. The properties of these maps will be treated next.

\subsubsection{Properties of Symplectic Maps}

In Lagrangian dynamics, the phase space of the system is defined by the set of functions $\left(\vec{q}, \frac{d \vec{q}}{d t}\right)$. In Hamiltonian dynamics, the phase space is parameterized by the

like an autonomous system. See Lanczos [68] for a discussion of this point.

${ }^{2}$ The geometric description of dynamical systems is a broad topic, and justice will not be done to it here. We defer to Arnold [60] for more complete details. 
set $(\vec{q}, \vec{p})$. In the latter case, the momenta are independent from the coordinates. An additional structure is needed to connect the coordinates with the momenta. This structure is called the symplectic condition.

\section{Symplectic condition and constraints}

The symplectic condition can be easily stated. A mapping $\phi$ is symplectic if its Jacobian is a symplectic matrix. We let $M$ denote the Jacobian matrix of the mapping $\phi(6.2)$,

$$
M_{a b}=\frac{\partial \zeta_{a}^{i}}{\partial \zeta_{b}^{f}},
$$

with transpose $\widetilde{M}$, and $J$ the $2 m \times 2 m$ symplectic form ${ }^{3}$,

$$
J=\left(\begin{array}{cc}
0 & I \\
-I & 0
\end{array}\right)
$$

where $I$ is the $m \times m$ unit matrix. The symplectic condition is then the nonlinear constraint

$$
\widetilde{M}\left(\zeta^{i}\right) J M\left(\zeta^{i}\right)=J
$$

A map $\phi$ that has a Jacobian matrix $M$ that is symplectic at all points is a symplectic map.

\section{Symplectic group of matrices}

Symplectic matrices demonstrate the properties that define a group [62]. If a matrix $M$ is symplectic, its inverse $M^{-1}$ can be shown to exist and to be symplectic. If the matrices $M_{1}$ and $M_{2}$ are symplectic, then $M_{1} M_{2}$ is also symplectic. The identity matrix can be shown to be symplectic. These properties define a group. The symplectic group $S p(2 m)$ is the set of all symplectic matrices of rank $2 m$. Additionally, we consider an exponential represention of a symplectic matrix sufficiently near the identity. If $S$ is a $2 m \times 2 m$ symmetic matrix, a symplectic matrix $M$ can be defined by

$$
M=\exp (J S)=\sum_{l=0}^{\infty} \frac{(J S)^{l}}{l !} .
$$

\footnotetext{
${ }^{3}$ The skew-symmetric form, $J$, plays the same role in symplectic geometry as the metric in Riemannian geometry. See [62] for more discussion.
} 
The converse is also true. An arbitrary symplectic matrix can be repesented in this exponential form.

\subsection{Abstract Lie Groups and Algebras ${ }^{4}$}

A Lie group is a topological group. That is, a group which is also a topological space, so that group operations are continuous. The topological space of a Lie group is also an analytic (differentiable) manifold, where group actions are analytic. In particular, we can express group operations near the identity by a power series expansion. In any local neighborhood, the manifold appears to be a Euclidean vector space. The tangent space to the manifold near the group identity element is called the Lie algebra. For a linear group, the algebra can be explicitly determined by differentiating curves that pass through the identity.

A Lie algebra $g$ is a linear vector space over a field $F$ (usually the real or complex numbers), where a vector space product [,] is defined. In addition to the requirements for a linear vector space, a Lie algebra must also satisfy:

1. Closure : $X, Y \in g \Rightarrow[X, Y] \in g$.

2. Bilinearity : $[X, a Y+b Z]=a[X, Y]+b[X, Z] ; X, Y, Z \in g, a, b \in F$.

3. Skew-symmetry : $[X, Y]=-[Y, X]$.

4. Jacobi identity : $[X,[Y, Z]]+[Y,[Z, X]]+[Z,[X, Y]]=0$.

A representation of a Lie algebra $g$ on a vector space $V$ is a mapping $\rho$ from $g$ to the set of linear transformations of $V$ such that

- $\rho(a X+b Y)=a \rho(X)+b \rho(Y)$

- $\rho([X, Y])=\{\rho(X), \rho(Y)\}=\rho(X) \rho(Y)-\rho(Y) \rho(X)$.

Here $\{$,$\} is the commutator, and [,] is the group product. A representation has the same$ dimension as the vector space $V$.

A special type of representation is formed if we define an operator $\operatorname{adX}$, where $X \in g$, that acts upon elements of the algebra $Y \in g$ via the group product, $a d X$ : $Y \rightarrow[X, Y]$. This operator is a linear transformation of $g$ into itself. The mapping

\footnotetext{
${ }^{4}$ This section borrows heavily from Sattinger and Weaver [70].
} 
$X \rightarrow a d X$ is a representation of the Lie algebra $g$ with $g$ itself playing the role of the vector space of transformations. This representation is called the adjoint representation. This representation always provides a matrix representation of the Lie algebra. To see this, consider a basis $\left\{E_{i}\right\}$ of the algebra. Then

$$
\operatorname{ad} E_{i}\left(E_{j}\right)=C_{i j}^{k} E_{k}
$$

(where $C_{i j}^{k}$ are the structure constants of the algebra), and

$$
\left(M_{i}\right)_{j k}=C_{i k}^{j}
$$

is an element of the operator matrix in the adjoint representation.

We have already discussed that a Lie algebra is the tangent space of the underlying Lie group manifold. This is formalized by theorem (see [70] for details). Let $G$ be a matrix Lie group, and let $g$ be the set of tangent vectors to all curves in $G$ at the identity. Then $g$ is a Lie algebra of matrices with the commutator as the Lie product. The converse is also true. For a linear Lie algebra $g$, the exponentials generate a Lie group $G$. Heuristically, $G(t)=\exp (t g)$. There is a much deeper result that any Lie algebra over the real or complex numbers generates a Lie group [70].

These same arguments can be made to apply to the symplectic mappings $\phi$. Hence, the set of symplectic maps defines a group, and this a Lie group with an associated Lie algebra. For symplectic matrices, the Lie algebra is finite dimensional. For symplectic mappings, the Lie algebra is infinite dimensional.

\subsection{Lie Algebraic Tools}

\subsubsection{Lie Operators and Lie Transformations}

A Lie operator can be constructed from any scalar function on the phase space, $f(\zeta)=$ $f(q, p)$, and the Poisson bracket, $[\cdot, \cdot]$. We denote this operator by $: f:$, and define it by the action of the Poisson bracket,

$$
\begin{aligned}
: f: & \doteq \sum_{j=1}^{n}\left(\frac{\partial f}{\partial q_{j}} \frac{\partial}{\partial p_{j}}-\frac{\partial f}{\partial p_{j}} \frac{\partial}{\partial q_{j}}\right) \\
& =[f, \cdot] .
\end{aligned}
$$


The action of this operator upon another function on the phase space, $g(q, p)$, is : $f$ : $g=[f, g]$. Powers of the Lie operator are defined by repeated operation with the Poisson bracket, for example

$$
: f:^{2} g=[f,[f, g]]
$$

while the zeroth power is taken to be the identity,

$$
: f:^{0} g=I g=g \text {. }
$$

The set of all phase space functions, $\{f(\zeta)\}$, forms a linear vector space, while the Poisson bracket satisfies the requirements for a Lie group product . Hence, the set of all phase space functions defines a Lie algebra with the Poisson bracket as the Lie product. This Lie algebra is infinite-dimensional, since the vector space of functions defined on the phase space is of infinite dimension. The set of all Lie operators forms an adjoint representation of this Lie algebra, also infinite-dimensional.

We can discuss power series of Lie operators, since we have defined powers of Lie operators. A Lie transformation is a particular power series, the exponentiation of a Lie operator, which produces a member of a Lie group,

$$
\exp (: f:)=\sum_{j=0}^{\infty} \frac{(: f:)^{j}}{j !} .
$$

This is a group of symplectic transformations on phase space.

These transformations are the final product we seek. If we consider a set of initial phase space coordinates, $\zeta^{i}$, and a function defined with them, $f\left(\zeta^{i}\right)$, then the coordinate transformation

$$
\zeta^{f}=\exp (: f:) \zeta^{i}
$$

can be shown to be symplectic. Hence, we can represent any symplectic mapping as a power series of linear operators,

$$
\phi \Leftrightarrow \exp (: f:)
$$

This shows the isomorphism that exists between Lie transformations and symplectic mappings. Since we already know that symplectic mappings can be used to study and solve problems in Hamiltonian dynamics, we are now assured that we can do the same thing with Lie transformations. In fact, Lie transformations provide us with an actual operator basis that can be used to compute the transfer map. 


\subsubsection{Useful Properties and Theorems of Lie Transformations}

There are several important properties of Lie transformations which will be useful for our purposes [67].

- Linearity - $\exp (: f:)(a g+b h)=a \exp (: f:) g+b \exp (: f:) h ; a, b \in R$.

- Isomorphism with respect to ordinary multiplication -

$$
\exp (: f:)(g h)=(\exp (: f:) g)(\exp (: f:) h)
$$

- Isomorphism with respect to Poisson brackets -

$$
\exp (: f:)[g, h]=[\exp (: f:) g, \exp (: f:) h]
$$

- Transformation of a function via its arguments -

$$
\exp (: f:) g\left(\zeta_{1}, \zeta_{2}, \ldots, \zeta_{2 m}\right)=g\left(\exp (: f:) \zeta_{1}, \ldots, \exp (: f:) \zeta_{2 m}\right)
$$

To construct a symplectic mapping via Lie transformation requires finding a suitable phase space function, $f(\zeta)$, as the generator. Since this generating function is a member of an infinite-dimensional linear vector space, we may analyze it with respect to any particular set of basis functions that span the vector space. We will choose to work with a monomial basis so that, in general, $f(\zeta)$ will be a homogeneous polynomial in the phase space coordinates. This will allow us to construct and analyze the resultant map in the linear approximation and order-by-order in nonlinearity.

\section{Factorization Theorem}

The mapping $\phi$ images the origin of phase space back onto itself. In this case, there is a theorem [71] that shows that $\phi$ can be uniquely represented by an infinite product of Lie transformations in the form

$$
\phi \Leftrightarrow \exp \left(: f_{1}:\right) \exp \left(: f_{2}:\right) \exp \left(: f_{3}:\right) \exp \left(: f_{4}:\right) \cdots,
$$

where each $f_{m}\left(\zeta^{i}\right)$ is a homogeneous polynomial of degree $m$ in the initial coordinates. A corollary to this theorem states that a unique reverse factorization also exists

$$
\phi \Leftrightarrow \cdots \exp \left(: g_{4}:\right) \exp \left(: g_{3}:\right) \exp \left(: g_{2}:\right) \exp \left(: g_{1}:\right)
$$


Both of these forms will be useful to us.

This theorem also shows that any set of homogeneous polynomials can be used to construct a symplectic mapping. We will take this to mean that we can produce a factorization which isolates the linear beam dynamics from second-order effects, thirdorder effects, etc. The product of factors can be terminated at any order and still produce a symplectic mapping. This is a very important result for our purposes. This allows us to construct a map that captures all the essential dynamics up to a predetermined order, neglecting all higher-order aberrations, and remains symplectic.

This illustrates our program of constructing maps according to the underlying Hamiltonian dynamics. The task of constructing a nonlinear, analytic mapping reduces to that of finding a distinct set of homogeneous polynomial functions. From the point of view of studying beam transport, the task is determine what polynomials are associated with what beamline component. To take overlapping fringe fields into account, however, we no longer think of the individual elements as isolated. We then look at the entire vector potential, and its power series expansion, to construct a set of polynomials order-byorder.

\section{Sandwich Theorem}

The sandwich theorem [69] gives a rule for exchanging the order of factors in the product of Lie transformations. If $: f:$ and $: g:$ are both Lie operators, then

$$
\exp (: f:) \exp (: g:)=\exp (: g:) \exp (: \exp (:-g:) f:)
$$

\section{CBH Theorem}

The Cambell-Baker-Hausdorff (CBH) theorem [70] shows up in quite many different contexts in mathematical physics. Its statement is simple enough. Consider two Lie operators, $: f:$ and $: g:$, sufficiently close to the identity. A third operator, denoted by $: h:$, and related to $: f:$ and $: g:$ by

$$
\exp (: h:)=\exp (: f:) \exp (: g:)
$$

is uniquely defined and is given by

$$
h=f+g+\frac{1}{2}[f, g]+\frac{1}{12}[f,[f, g]]+\frac{1}{12}[g,[g, f]]+\cdots .
$$


This gives us a rule for combining terms in the factored product. This is a very remarkable theorem from the result that the right-hand side is solely composed of nested Poisson brackets.

\section{Truncation and Rank}

The factored product representation of the mapping (6.9) contains an infinite number of terms. Obviously, we will want to truncate that product at some point. The issue arises, then, of how to do so while retaining the Lie algebraic structure.

Consider an homogeneous polynomial of order $m, f_{m}(\zeta)$. We assign an integer to this polynomial, called the rank of the polynomial, equal to its order,

$$
\operatorname{rank} f_{m}=m \text {. }
$$

We also assign a value to the result of a Poisson bracket,

$$
\operatorname{rank}\left[f_{m}, f_{n}\right]=m+n-2 .
$$

The set of all polynomials with rank $\geq 2$ forms an infinite-dimensional Lie algebra with the Poisson bracket as the Lie operator. By discarding all polynomials and Poisson bracket results with rank greater than some value $N$, we are left with an $N$-dimensional quotient Lie algebra. Exponentiating the elements in the quotient algebra yields members of the quotient group. Carrying this process over to Lie operators, by neglecting all operators : $f_{m}$ : in which $\operatorname{rank} f_{m}>N$, we construct an $N$-dimensional quotient Lie algebra of Lie operators. This provides a systematic method of limiting the order of any calculation while still maintaining strict symplecticity.

Getting back to beam dynamics, once we decide to ignore optical aberrations greater than some degree $N-1$, we then ignore all algebraic objects with rank $>N$. We may then truncate the factored product at $\exp \left(: f_{N}:\right)$,

$$
\phi \Leftrightarrow \exp \left(: f_{1}:\right) \exp \left(: f_{2}:\right) \exp \left(: f_{3}:\right) \exp \left(: f_{4}:\right) \cdots \exp \left(: f_{N}:\right)
$$

\subsection{Symbolic Computation of Maps}

In this section, we will discuss the explicit calculation of the equations of motion of the mapping $\phi$, induced by the Hamiltonian $H(\zeta, t)$. We consider a mapping interval with some well-defined initial coordinates $\zeta^{i}$ and initial time $t^{i}$, but with variable final time $t$ 
and final coordinates $\zeta$. The final coordinates are formally computed by

$$
\zeta(t)=\phi \zeta^{i}
$$

We consider an arbitrary function on phase space $f(\zeta)$. From the properties of Lie transfromations we know that $\phi$ has the property

$$
f(\zeta)=f\left(\phi \zeta^{i}\right)=\phi f\left(\zeta^{i}\right)
$$

Taking the time derivative along a trajectory,

$$
\frac{d f}{d t}=\frac{d \phi}{d t} f\left(\zeta^{i}\right)
$$

The equations of motion for $f$ are given by the Poisson bracket relation,

$$
\begin{aligned}
\frac{d f}{d t} & =-[H(\zeta, t), f(\zeta)] \\
& =-\left[H\left(\phi \zeta^{i}, t\right), f\left(\phi \zeta^{i}\right)\right] \\
& =-\left[\phi H\left(\zeta^{i}, t\right), \phi f\left(\zeta^{i}\right)\right] \\
& =-\phi\left[H\left(\zeta^{i}, t\right), f\left(\zeta^{i}\right)\right] \\
& =\phi:-H\left(\zeta^{i}, t\right): f\left(\zeta^{i}\right) .
\end{aligned}
$$

Comparing this to the expression above, we obtain

$$
\frac{d f}{d t}=\frac{d \phi}{d t} f\left(\zeta^{i}\right)=\phi:-H\left(\zeta^{i}, t\right): f\left(\zeta^{i}\right)
$$

The function $f$ is arbitrary. The mapping must then obey the equation of motion

$$
\frac{d \phi}{d t}=\phi:-H\left(\zeta^{i}, t\right):
$$

We previously calculated a power series representation of the deviation Hamiltonian to fourth-order. That is, we assume that we have a Hamiltonian in the form

$$
H(\zeta, t)=H_{2}(\zeta, t)+H_{3}(\zeta, t)+H_{4}(\zeta, t)=H_{2}+H_{R}
$$

where each term $H_{m}$ is a sum of homogenous polynomials of order $m$. We expect, then, 
to obtain a mapping given in the factorized form

$$
\begin{aligned}
\phi & =\phi_{4} \phi_{3} \phi_{2}=\phi_{R} \phi_{2} \\
& =\exp \left(: h_{4}:\right) \exp \left(: h_{3}:\right) \exp \left(: h_{2}:\right)=\exp \left(: h_{R}:\right) \exp \left(: h_{2}:\right)
\end{aligned}
$$

where each $h_{m}$ is again a sum of homogeneous polynomials. In the above we have separated the linear dynamics (order 2), from the higher-order remainder terms (labeled by $R$ ). The equation of motion for the mapping can then be expressed as

$$
\begin{aligned}
\frac{d \phi}{d t} & =\frac{d \phi_{R}}{d t} \phi_{2}+\phi_{R} \frac{d \phi_{2}}{d t} \\
& =\phi_{R} \phi_{2}:-H_{2}-H_{R}: \\
& =\phi_{R} \phi_{2}:-H_{2}:+\phi_{R} \phi_{2}:-H_{R}: .
\end{aligned}
$$

Now, it can be shown [71] that $\phi_{2}$ obeys the equation of motion,

$$
\frac{d \phi_{2}}{d t}=\phi_{2}:-H_{2}:
$$

It follows that the remainder term must obey,

$$
\frac{d \phi_{R}}{d t}=\phi_{R} \phi_{2}:-H_{R}: \phi_{2}^{-1}
$$

The factor on the right-hand side can be shown to be the interaction Hamiltonian - that is, the Hamiltonian remaining when the dynamics is viewed in the interaction picture. This transformation is familiar from studies of the nonlinear harmonic oscillator in perturbation theory [72] — we change frames in phase space to one that rotates with the same angular frequency as the unperturbed oscillator. In this case, we examine the nonlinear Hamiltonian obtained after we change its dependent variables to solutions of the linear equations of motion. That is,

$$
\begin{aligned}
H_{R}^{i n t}\left(\zeta^{i}, t\right) & =\phi_{2} H_{R}\left(\zeta^{i}, t\right) \\
& =H_{R}\left(\phi_{2} \zeta^{i}, t\right)
\end{aligned}
$$

The equation of motion for the higher-order mapping is then

$$
\frac{d \phi_{R}}{d t}=\phi_{R}:-H_{R}^{i n t}: .
$$




\section{Linear mapping}

The linear part of the dynamics, governed by $\phi_{2}$, has a simple matrix representation. If we denote by $M$ the transformation matrix, then

$$
\bar{\zeta}^{f}=\phi_{2} \zeta^{i}=M \zeta^{i}
$$

and in particular,

$$
\left(\bar{\zeta}^{f}\right)_{a}=M_{a b}\left(\zeta^{i}\right)_{b}
$$

Now, the second-order Hamiltionan can be written in the form

$$
H_{2}\left(\zeta^{i}, t\right)=\frac{1}{2} \sum_{a, b} S_{a b}(t) \zeta_{a}^{i} \zeta_{b}^{i}
$$

where $S$ is a symmetric matrix. We next compute the quantity $:-H_{2}: \zeta^{i}$, obtaining

$$
:-H_{2}:\left(\zeta^{i}\right)_{c}=\left[-H_{2},\left(\zeta^{i}\right)_{c}\right]=\sum_{a, b} J_{c a} S_{a b}\left(\zeta^{i}\right)_{b}=\left(J S \zeta^{i}\right)_{c}
$$

where the symplectic form has been defined by the fundamental Poisson brackets,

$$
J_{a b} \doteq\left[\left(\zeta^{i}\right)_{a},\left(\zeta^{i}\right)_{b}\right]
$$

We apply (6.17) to an initial vector

$$
\begin{aligned}
\frac{d \phi_{2}}{d t} \zeta^{i} & =\phi_{2}:-H_{2}: \zeta^{i} \\
& =\phi_{2} J S \zeta^{i} \\
& =J S \phi_{2} \zeta^{i} \\
& =J S M \zeta^{i} .
\end{aligned}
$$

The left-hand side of the above equation is simply

$$
\frac{d \phi_{2}}{d t} \zeta^{i}=\frac{d M}{d t} \zeta^{i}
$$

Since the vector $\zeta^{i}$ is arbitrary, we are left with the equation of motion for the linear portion of the mapping,

$$
\frac{d M}{d t}=J S M
$$




\section{Interaction Hamiltonian}

The interaction Hamiltonian can be calculated immediately. To fourth-order we have,

$$
H_{R}=H_{3}+H_{4},
$$

so that

$$
\begin{aligned}
H_{R}^{i n t}\left(\zeta^{i}, t\right) & =H_{3}^{i n t}\left(\zeta^{i}, t\right)+H_{4}^{i n t}\left(\zeta^{i}, t\right) \\
& =H_{3}\left(\phi_{2} \zeta^{i}, t\right)+H_{4}\left(\phi_{2} \zeta^{i}, t\right) \\
& =H_{3}\left(M \zeta^{i}, t\right)+H_{4}\left(M \zeta^{i}, t\right) \\
& =H_{3}\left(\bar{\zeta}^{f}, t\right)+H_{4}\left(\bar{\zeta}^{f}, t\right) .
\end{aligned}
$$

\section{Higher-order Lie polynomials}

The calculation of the higher-order polynomials, $h_{3}$ and $h_{4}$, in (6.16) is somewhat more involved. Details may be found in [71]. Using the factorization order

$$
\phi=\exp \left(: h_{4}:\right) \exp \left(: h_{3}:\right) \exp \left(: h_{2}:\right)
$$

they find that these polynomials are determined from the equations

$$
\begin{gathered}
h_{3}=-\int_{t^{i}}^{t} d t^{\prime} H_{3}^{i n t}\left(\zeta^{i}, t^{\prime}\right), \\
h_{4}=-\int_{t^{i}}^{t} d t^{\prime} H_{4}^{i n t}\left(\zeta^{i}, t^{\prime}\right)+\frac{1}{2} \int_{t^{i}}^{t} d t^{\prime} \int_{t^{i}}^{t^{\prime}} d t^{\prime \prime}\left[-H_{3}^{i n t}\left(\zeta^{i}, t^{\prime \prime}\right),-H_{3}^{i n t}\left(\zeta^{i}, t^{\prime}\right)\right] .
\end{gathered}
$$

These forms are useful for analytical work. For numerical work, the equivalent set of equations more convenient to use are

$$
\begin{gathered}
\frac{d h_{3}}{d t}=-H_{3}^{i n t} \\
\frac{d h_{4}}{d t}=-H_{4}^{i n t}+\frac{1}{2}: h_{3}: H_{3}^{i n t} .
\end{gathered}
$$

The equations $(6.19),(6.20),(6.21)$ form a set of coupled, nonlinear, ordinary differential equations that we must solve to calculate the transfer maps. The initial conditions on this set is determined by considering the action of the mapping with a zero time interval. In this case, the mapping must be the identity. Hence, $M\left(t=t^{i}\right)=I$, while $h_{3}=h_{4}=0$ 
at $t=t^{i}$.

\section{Rules for successive transformation}

Having found the linear mapping, $M$, as well as the higher-order Lie polynomials $h_{3}$ and $h_{4}$, we must still evaluate the action of the maps upon initial coordinate vector $\zeta^{i}$. That is, we must evaluate

$$
\phi \zeta^{i}=\exp \left(: h_{4}:\right) \exp \left(: h_{3}:\right) \exp \left(: h_{2}:\right) \zeta^{i}
$$

Before doing that, however, we must first determine how to calculate the effect of successive Lie transformations. We will consider two mappings

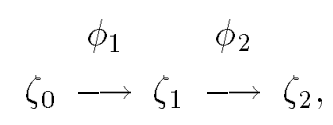

where

$$
\begin{aligned}
\phi_{1} & \Leftrightarrow \exp \left(: f_{1}:\right), \\
\phi_{2} & \Leftrightarrow \exp \left(: f_{2}:\right) .
\end{aligned}
$$

Writing this series explicitly, we have

$$
\begin{aligned}
& \zeta_{1}=\phi_{1}\left(\zeta_{0}\right) \zeta_{0}, \\
& \zeta_{2}=\phi_{2}\left(\zeta_{1}\right) \zeta_{1},
\end{aligned}
$$

and

$$
\zeta_{2}=\phi_{2}\left(\zeta_{1}\right) \phi_{1}\left(\zeta_{0}\right) \zeta_{0}
$$

The latter occuring transformation acts upon the results of the previous transformation. The arguments of these two analytic mappings are not identical. To proceed, we need to determine the correct way of calculating these transformations so that the initial vector $\zeta_{0}$ is the sole input. We proceed by expanding the argument in the 'later' transformation

$$
\begin{aligned}
\zeta_{2} & =\exp \left(: f_{2}\left(\zeta_{1}\right):\right) \exp \left(: f_{1}\left(\zeta_{0}\right):\right) \zeta_{0} \\
& =\exp \left(: f_{2}\left(\exp \left(: f_{1}\left(\zeta_{0}\right):\right) \zeta_{0}\right):\right) \exp \left(: f_{1}\left(\zeta_{0}\right):\right) \zeta_{0} \\
& =\exp \left(: \exp \left(: f_{1}\left(\zeta_{0}\right):\right) f_{2}\left(\zeta_{0}\right):\right) \exp \left(: f_{1}\left(\zeta_{0}\right):\right) \zeta_{0}
\end{aligned}
$$




$$
\begin{aligned}
& =\exp \left(: f_{1}\left(\zeta_{0}\right):\right) \exp \left(: f_{2}\left(\zeta_{0}\right):\right) \exp \left(:-f_{1}\left(\zeta_{0}\right):\right) \exp \left(: f_{1}\left(\zeta_{0}\right):\right) \zeta_{0} \\
& =\exp \left(: f_{1}\left(\zeta_{0}\right):\right) \exp \left(: f_{2}\left(\zeta_{0}\right):\right) \zeta_{0} \\
& =\phi_{1}\left(\zeta_{0}\right) \phi_{2}\left(\zeta_{0}\right) \zeta_{0} .
\end{aligned}
$$

To derive the third line from the second we used the rule for transformation of a function via its argument, and to derive the fourth we used the sandwich theorem. We see that the net action of successive Lie transformations is to apply them in anti-chronological order onto the initial coordinate vector. Thus, the latter occuring tranformations are evaluated before the prior ones.

In deriving the differential equations governing the evolution of the mappings (6.19 - 6.21), it was convenient to use the particular factorization order

$$
\phi=\cdots \exp \left(: h_{4}:\right) \exp \left(: h_{3}:\right) \exp \left(: h_{2}:\right)
$$

However, when we actually wish to advance a set of coordinate vectors with this mapping, we find that the operator ordering is opposite to that which we want ${ }^{5}$. We know that an equivalent factorization

$$
\phi=\exp \left(: g_{2}:\right) \exp \left(: g_{3}:\right) \exp \left(: g_{4}:\right) \cdots
$$

exists. The task is to relate the set of Lie polynomials we desire $\left\{g_{i}\right\}$ to those we have computed $\left\{h_{i}\right\}$, and to calculate the net transformation from the factored product.

We begin by inserting several identity factors, defined by

$$
I=\exp \left(: h_{2}:\right) \exp \left(:-h_{2}:\right)
$$

into the expansion,

$$
\begin{aligned}
\phi= & \exp \left(: h_{4}:\right) \exp \left(: h_{3}:\right) \exp \left(: h_{2}:\right) \\
= & \exp \left(: h_{2}:\right) \exp \left(:-h_{2}:\right) \exp \left(: h_{4}:\right) \exp \left(: h_{2}:\right) \exp \left(:-h_{2}:\right) \times \\
& \times \exp \left(: h_{3}:\right) \exp \left(: h_{2}:\right) \exp \left(:-h_{2}:\right) \exp \left(: h_{2}:\right)
\end{aligned}
$$

From the sandwich theorem, factors can be grouped together like

$$
\exp \left(:-h_{2}:\right) \exp \left(: h_{4}:\right) \exp \left(: h_{2}:\right)=\exp \left(: \exp \left(:-h_{2}:\right) h_{4}:\right)
$$

\footnotetext{
${ }^{5}$ This point is discussed further in [73].
} 
The mapping then reads as

$$
\begin{aligned}
\phi & =\exp \left(: h_{2}:\right) \exp \left(: \exp \left(:-h_{2}:\right) h_{4}:\right) \exp \left(: \exp \left(:-h_{2}:\right) h_{3}:\right) \\
& =\exp \left(: h_{2}:\right) \exp \left(: h_{4}^{\prime}:\right) \exp \left(: h_{3}^{\prime}:\right)
\end{aligned}
$$

Next, invoke the $\mathrm{CBH}$ theorem to rearrange the right-hand side

$$
\begin{aligned}
\exp \left(: h_{4}^{\prime}:\right) \exp \left(: h_{3}^{\prime}:\right) & =\exp \left(:\left\{h_{4}^{\prime}+h_{3}^{\prime}+\frac{1}{2}\left[h_{4}^{\prime}, h_{3}^{\prime}\right]+\cdots\right\}:\right) \\
& =\exp \left(: h_{3}^{\prime}:\right) \exp \left(:\left\{h_{4}^{\prime}+\frac{1}{2}\left[h_{4}^{\prime}, h_{3}^{\prime}\right]+\cdots\right\}:\right)
\end{aligned}
$$

This, then, is our reverse factorization

$$
\begin{aligned}
& g_{2}=h_{2}, \\
& g_{3}=h_{3}^{\prime}=\exp \left(:-h_{2}:\right) h_{3} \\
& g_{4}=h_{4}^{\prime}+\frac{1}{2}\left[h_{4}^{\prime}, h_{3}^{\prime}\right]+\cdots=\exp \left(:-h_{2}:\right) h_{4}+\frac{1}{2} \exp \left(:-h_{2}:\right)\left[h_{4}, h_{3}\right]+\cdots
\end{aligned}
$$

Next, we evaluate the action of the mapping upon the initial coordinate vector,

$$
\zeta^{f}=\exp \left(: g_{2}:\right) \exp \left(: g_{3}:\right) \exp \left(: g_{4}:\right) \zeta^{i}
$$

The exponential factors are functional representations of an infinite power series of Lie operators. To explicitly evaluate these factors, we must expand each power series, and retain only those terms with rank $<4$. In effect, we are reproducing the original truncated Taylor series (6.1). This has the effect of violating strict symplecticity. In fact, the resulting transformation will only be symplectic up to the order at which we truncate the series. As already said, this is not a serious error for single-pass beamlines, and we will still greatly benefit from the economy of calculation by using the Lie series representation.

The linear transformation has been explicitly calculated to be

$$
\exp \left(: g_{2}:\right)=\exp \left(: h_{2}:\right)=M
$$

where $M$ is the matrix representation of the linear portion of the operator. The higherorder-polynomial exponential factors are expanded in a Taylor series, and truncated at 
the appropriate $r a n k$,

$$
\begin{aligned}
& \exp \left(: g_{3}:\right)=1+: g_{3}:+\frac{1}{2}: g_{3}:^{2}+O(\text { rank }=4) \\
& \exp \left(: g_{4}:\right)=1+: g_{4}:+O(\operatorname{rank}=5)
\end{aligned}
$$

Retaining terms to $r a n k=3$, the approximate mapping is

$$
\begin{aligned}
\phi & \approx \exp \left(: g_{2}:\right)\left\{1+: g_{3}:+\frac{1}{2}: g_{3}:^{2}\right\}\left\{1+: g_{4}:\right\} \\
& =\exp \left(: g_{2}:\right)\left\{1+: g_{3}:+\frac{1}{2}: g_{3}:^{2}+: g_{4}:\right\}
\end{aligned}
$$

Finally, we replace the set $\left\{g_{i}\right\}$ with their values determined from the set $\left\{h_{i}\right\}$,

$$
\begin{aligned}
\phi \approx & \exp \left(: g_{2}:\right)\left\{1+: g_{3}:+\frac{1}{2}: g_{3}:^{2}+: g_{4}:\right\} \\
= & \exp \left(: g_{2}:\right)\left\{1+: \exp \left(:-h_{2}:\right) h_{3}:+\frac{1}{2}: \exp \left(:-h_{2}:\right) h_{3}:^{2}+\right. \\
& \left.+: \exp \left(:-h_{2}:\right) h_{4}:+\frac{1}{2}: \exp \left(:-h_{2}:\right)\left[h_{4}, h_{3}\right]:\right\} \\
= & M+\left[h_{3}, M\right]+\frac{1}{2}\left[h_{3},\left[h_{3}, M\right]\right]+\left[h_{4}, M\right]+O(\text { rank }=4)
\end{aligned}
$$

The transformed coordinate vector is then

$$
\zeta^{f} \approx \bar{\zeta}+\left[h_{3}, \bar{\zeta}\right]+\frac{1}{2}\left[h_{3},\left[h_{3}, \bar{\zeta}\right]\right]+\left[h_{4}, \bar{\zeta}\right]+O\left(\left(\zeta^{i}\right)^{4}\right)
$$

where $\bar{\zeta}=M \zeta^{i}$. This expansion thus yields solutions valid to 3rd order in powers of the deviation coordinates. As previously discussed in section 6.4.2, the function $h_{3}$ and $h_{4}$ are homogeneous polynomials (or degree 3 and 4 , respectively) in the phase space deviation coordinates, (5.5). The third-order polynomial, $h_{3}$, has 56 terms and the fourth-order polynomial, $h_{4}$, has 126 terms. The linear mapping is described by the $6 \times 6$ matrix, $M$, which has 21 independent elements. The zeroth order dynamic is represented in the fiducial orbit which has 6 elements (the phase space coordinates). Altogether, this represents 209 independent coefficients that must be calculated for a third-order description of the beam dynamics.

This is the main result of this chapter. It expresses the division of the mapping into linear order (first term on the right hand side), second-order (second term), and third-order (third and fourth terms). This mapping algorithm is utilized for advancing 
particles in the external fields of the beamline elements.

\subsection{Symplectic Numerical Integration}

The previous section culminated in an algorithm for calculating maps to represent the advance of an ensemble of particles across a given beamline interval. For the single particle (noninteracting) Hamiltonian (5.2), that is the end of the calculation. However, to account for the effects of self-fields (in the electrostatic approximation), we must return to the original Hamiltonian (5.1). In this case, we have a Hamiltonian system composed of two distinct terms, representing very different dynamics. Assuming that we could handle each of these terms as bona fide Hamiltonians individually, we still require a method for incorporating their actions into a joint description of the total dynamics of the system. This can be done by utilizing the so-called symplectic integrators.

Symplectic integrators [74], [75], [76], [77] are algorithms used to numerically integrate systems of differential equations, which have been derived from Hamiltonian functions on some even-dimensional phase space. These algorithms create an approximation to the solution of the differential equations, by constructing an approximate symplectic mapping that is valid for a short time-step. A $k^{t h}$-order symplectic integrator creates a mapping $\phi^{(k)}(t)$ which approximates the exact mapping $\phi(t)$ through order $t^{k}$. The difference between the two maps will then be of order $t^{k+1}$,

$$
\left\|\phi(t)-\phi^{(k)}(t)\right\|=O\left(t^{k+1}\right) .
$$

The symplectic integrator scheme has its greatest utility when we can express the (possibly non-integrable) Hamiltonian function, $H(\zeta)$, as a sum of independent, integrable pieces $^{6}$,

$$
H(\zeta)=H_{1}(\zeta)+H_{2}(\zeta)+\cdots
$$

Recall the equation of motion of an arbitrary mapping (6.15) derived from a timeindependent Hamiltonian,

$$
\frac{d \phi}{d t}=\phi:-H\left(\zeta^{i}\right):
$$

We formally integrate this equation to arrive at our solution,

$$
\phi(t)=\exp \left(:-t H\left(\zeta^{i}\right):\right)
$$

\footnotetext{
${ }^{6}$ That is, the equations of motion derived from the individual $H_{i}(\zeta)$ may be solved exactly by analytical methods, or numerically to arbitrary precision.
} 
With a Hamiltonian expressed in the form (6.23), we have terms that are exactly solvable. Let these solutions be expressed as

$$
\phi_{\ell}(t)=\exp \left(:-t H_{\ell}\left(\zeta^{i}\right):\right), \ell=1,2, \ldots
$$

We now wish to construct an approximation to the total mapping (6.24) by using the exact, piecewise mappings (6.25). To do this we need only appeal to the Lie algebraic structure of the symplectic maps. In particular, we will utilize the CBH theorem.

As an illustration of this method, we follow the discussion in [75] and construct an explicit, $2^{\text {nd }}$-order in time symplectic integrator, for a two-map system (ie. the total Hamiltonian in (6.23) which can be written as the sum of two integrable pieces). Consider first, the following approximation to the total map,

$$
\begin{aligned}
\tilde{\phi}(t) & =\phi_{1}(t) \phi_{2}(t) \\
& =\exp \left(:-t H_{1}:\right) \exp \left(:-t H_{2}:\right) \\
& =\exp \left(:-t H-\frac{1}{2} t^{2}\left[H_{1}, H_{2}\right]+\cdots:\right) \\
& =\phi(t)+O\left(t^{2}\right) \\
& =\phi^{(1)}(t) .
\end{aligned}
$$

This approximation evidently leads to a $1^{\text {st }}$-order integrator. Next, consider a symmetrized version of the approximate map,

$$
\begin{aligned}
\widetilde{\phi}(t) & =\phi_{1}\left(\frac{1}{2} t\right) \phi_{2}(t) \phi_{1}\left(\frac{1}{2} t\right) \\
& =\exp \left(:-\frac{1}{2} t H_{1}:\right) \exp \left(:-t H_{2}:\right) \exp \left(:-\frac{1}{2} t H_{1}:\right) \\
& =\exp \left(:-t\left(\frac{1}{2} H_{1}+H_{2}\right)-\frac{1}{4} t^{2}\left[H_{1}, H_{2}\right]+\cdots:\right) \exp \left(:-\frac{1}{2} t H_{1}:\right) \\
& =\exp \left(:-t\left(H_{1}+H_{2}\right)-\frac{1}{4} t^{2}\left[H_{1}, H_{2}\right]-\frac{1}{4} t^{2}\left[H_{2}, H_{1}\right]+O\left(t^{3}\right):\right) \\
& =\exp \left(:-t H+O\left(t^{3}\right):\right) \\
& =\phi^{(2)}(t) .
\end{aligned}
$$

So, by symmetrizing the order of the operators, we can construct a higher-order integrator (in this case, a $2^{\text {nd }}$-order integrator). This is actually a general result - Yoshida [76] proves that any symmetrized symplectic integrator is automatically of even order. 
This technique is useful whenever a set of distinct maps can be constructed. It makes no difference, how those maps are constructed, only that they are derivable from a Hamiltonian system, and that they are constructed to at least one higher order of approximation than the symplectic algorithm. In particular, we will use the algorithm (6.26) when we wish to combine the effects of single particle transport in external fields with the self-field interaction of the collection of particles in the beampipe. The selffield effects are represented by an impulse map, and are generated by a particle-in-cell module. This is not an explicitly Hamiltonian calculation, but it is equivalent to one, so that the impulse map may be utilized as a component in the symplectic integration. 


\section{Chapter 7}

\section{Beamline Element Description}

\subsection{Introduction}

In forming the equations of motion for the beam particles, we used a Hamiltonian formulation that explicitly uses potentials instead of fields. We assumed that the scalar potential could be neglected in favor of the vector potential. This amounts to a particular choice of gauge. Here, we make these assumptions a concrete part of the model of the physical environment. We consider those fields that arise from charge and current dstributions external to the beam, but which exert forces upon the beam particles. All of these sources will either be explicitly static in nature (eg. magnetic focusing elements, electrostatic induction modules), or quasi-static in approximation ( $\mathrm{rf}$ cavities). In the case of rf cavities, we will assume that the time-dependence of the fields during the transit-time of the bunch is harmonic with well-defined frequency and amplitude. We will develop a means of describing these fields from a static potential representation, ensuring conformance with Maxwell's equations, and allowing for a power series representation.

The particular gauge of choice for our potentials is the so-called transverse gauge [78]. In this gauge, we enforce (sometimes by explicit construction) the constraints

$$
\begin{aligned}
\Phi & =0, \\
\vec{\nabla} \cdot \vec{A} & =0 .
\end{aligned}
$$

The electric and magnetic fields in this gauge are given (in MKSA units) by

$$
\vec{E}=-\partial_{t} \vec{A}
$$




$$
\vec{B}=\vec{\nabla} \times \vec{A}
$$

Additionally, the gauge condition implies that in source-free regions the vector potential satisfies the vector Laplace equation,

$$
\nabla^{2} \vec{A}=0
$$

\section{On-axis expansion}

For the elements considered here, the region of interest — the beamline — is source-free by definition. We will find solutions to the vector Laplace equation in a circular cylindrical coordinate system $(r \varphi z)$, before converting to rectangular Cartesian coordinates (xyz). The coordinate $z$ we take to be the local element axis (and here we will require that it coincide with the mechanical beamline axis). In cylindrical coordinates, the vector Laplace equation is equivalent to the set of coupled partial differential equations

$$
\begin{aligned}
\nabla^{2} A_{z} & =0, \\
\nabla^{2} A_{r}-\frac{2}{r^{2}} \partial_{\varphi} A_{\varphi}-\frac{1}{r^{2}} A_{r} & =0, \\
\nabla^{2} A_{\varphi}+\frac{2}{r^{2}} \partial_{\varphi} A_{r}-\frac{1}{r^{2}} A_{\varphi} & =0,
\end{aligned}
$$

where

$$
\nabla^{2}=\frac{1}{r} \partial_{r}\left(r \partial_{r}\right)+\frac{1}{r^{2}} \partial_{\varphi \varphi}+\partial_{z z}
$$

is the scalar Laplacian operator. The divergence-less criterion is

$$
\frac{1}{r} \partial_{r}\left(r A_{r}\right)+\frac{1}{r} \partial_{\varphi}\left(A_{\phi}\right)+\partial_{z}\left(A_{z}\right)=0
$$

We solve this set of equations for each beamline element.

We will find solutions to (7.1) by multipole expansion. This is realized by first performing a Fourier series analysis (in $\varphi$ ) of each vector potential component (indexed by $j$ ),

$$
A_{j}(r, \varphi, z)=\sum_{m=0}^{\infty}\left[a_{j m}(r, z) \cos m \varphi+b_{j m}(r, z) \sin m \varphi\right] .
$$

Symmetry arguments are then used to identify the specific terms in the Fourier series which serve to define the principal multipole component(s) associated with a given beamline element. The Fourier coefficients are then expanded in a power series in $r$ about the 
axis, for example

$$
a_{j m}(r, z)=r^{\nu} \sum_{l=0}^{\infty} r^{l} a_{j m l}(z),
$$

which is used to analyze the set of PDE's. Elementary analytical techniques (e.g., Frobenius' method, see [79] for example) are applied to solve the set. The end result is a system that uses a single, analytic function (the multipole component along the beamline, for example) defined on the axis to generate the vector potential values everywhere else. This primitive function is given by suitable analytic formulae or, more generally, by data taken from measurements or numerical simulations of the physical device fields.

\section{Expansion about off-axis fiducial orbit}

In the situations where the fiducial orbit has a finite off-axis excursion, the power series developed so far must be modified. This is because we are seeking a power series expansion about the fiducial orbit. This can be accomplished in the Cartesian representation of the vector potential,

$$
A(x, y, z)=\sum_{i=0}^{\infty} \sum_{j=0}^{i} a_{i j}(z) x^{i-j} y^{j} .
$$

We form the difference of vector potentials observed at $(x, y, z)$ with those observed at the fiducial orbit coordinates $\left(x_{g}, y_{g}, z\right)$, and express them in terms of the transverse deviation coordinates,

$$
\begin{aligned}
\widehat{A}(x, y, z) & =A(x, y, z)-A\left(x_{g}, y_{g}, z\right) \\
& =\sum_{i=0}^{\infty} \sum_{j=0}^{i} a_{i j}(z)\left(x^{i-j} y^{j}-x_{g}^{i-j} y_{g}^{j}\right) \\
& =\sum_{i=0}^{\infty} \sum_{j=0}^{i} b_{i j}(z)\left(x-x_{g}\right)^{i-j}\left(y-y_{g}\right)^{j}
\end{aligned}
$$

The coefficients $b_{i j}$ can be related to the on-axis expansion coefficients $a_{i j}$.

\subsection{Magnetostatic Expansion}

The magnetostatic beamline elements we will encounter are permanent magnet quadrupoles, DC powered solenoids, and pulsed (but approximately DC) helical wigglers. The quadrupoles and solenoids are ubiquitous in accelerators and transport lines since they provide lowestorder transverse confinement and focusing of an otherwise expanding beam. We will form 
solutions to (7.1) consistent with the rotational symmetry of the individual element, by performing a multipole expansion valid in the interior region $(r \geq 0)$. However, we will not assume that each element is a perfect multipole with rigid boundaries. The physical beamline elements often employed have a high diameter-to-length ratio, and as such have significant fringe fields. This destroys the perfect multipole symmetry, by introducing higher-order pseudo-multipoles that are dependent upon the longitudinal derivative of the lower-order principal multipole components. This will be treated in the descriptions of the elements.

\subsubsection{Quadrupole}

To lowest-order, the magnetic field of a normal $^{1}$ quadrupole is given by [80]

$$
\begin{aligned}
& B_{x}=g y, \\
& B_{y}=g x,
\end{aligned}
$$

where $g$ is the quadrupole gradient. Since the field is predominantly transverse, the vector potential will be predominantly axial at lowest-order. A pure quadrupole field must exhibit two-fold symmetry in the transverse $(r \varphi)$-plane. A normal field will also exhibit even parity in $(\varphi)$, while a skew field will exhibit odd parity.

The axial vector potential of a normal quadrupole must then be of the form

$$
A_{z}(r, \varphi, z)=a(r, z) \cos 2 \varphi \text {. }
$$

Using a power series expansion in $r$ for $a(r, z)$, the $z$-component of $(7.1)$ has the solution

$$
A_{z}=\cos 2 \varphi \sum_{l=0}^{\infty} r^{2 l+2} a_{2 l}(z)
$$

where $a_{2 l}(z)$ obeys the recursion relation defined by

$$
a_{2 l}=-\frac{a_{2 l-2}^{\prime \prime}}{4\left(l^{2}+2 l\right)}, l \geq 1
$$

Throughout, primes will denote total differentiation with respect to the $z$ coordinate. The series expansion coefficients, $a_{l}(z)$, are identically zero for odd $l$.

\footnotetext{
${ }^{1}$ A skew quadrupole filed can be obtained from the normal field by a rotation of 45 degrees about the $\mathrm{z}$-axis.
} 
The transverse components of $\vec{A}$ will have similar expansions. The forms of these expansions can be determined by symmetry and the divergence-less criterion of the gauge vector potential to be

$$
\begin{aligned}
& A_{r}=\cos 2 \varphi \sum_{l=0}^{\infty} r^{2 l+3} b_{2 l}(z), \\
& A_{\varphi}=\sin 2 \varphi \sum_{l=0}^{\infty} r^{2 l+3} c_{2 l}(z) .
\end{aligned}
$$

These forms are substituted into the transverse components of (7.1). After some tedious algebra, we obtain

$$
\begin{aligned}
& A_{z}=\cos 2 \varphi \sum_{l=0}^{\infty} r^{2 l+2} a_{2 l}(z), \\
& A_{r}=-\cos 2 \varphi \sum_{l=0}^{\infty} r^{2 l+3} \frac{a_{2 l}^{\prime}(z)}{2 l+6}, \\
& A_{\varphi}=-\sin 2 \varphi \sum_{l=0}^{\infty} r^{2 l+3} \frac{a_{2 l}^{\prime}(z)}{2 l+6}, \\
& a_{2 l}=-\frac{a_{2 l-2}^{\prime \prime}}{4\left(l^{2}+2 l\right)}, l \geq 1 .
\end{aligned}
$$

In Cartesian coordinates, this expansion becomes

$$
\begin{aligned}
& A_{z}=\left(x^{2}-y^{2}\right) \sum_{l=0}^{\infty}\left(x^{2}+y^{2}\right)^{l} a_{2 l}(z) \\
& A_{x}=\left[-x^{3}+3 x y^{2}\right] \sum_{l=0}^{\infty}\left(x^{2}+y^{2}\right)^{l} \frac{a_{2 l}^{\prime}(z)}{2 l+6} \\
& A_{y}=\left[y^{3}-3 x^{2} y\right] \sum_{l=0}^{\infty}\left(x^{2}+y^{2}\right)^{l} \frac{a_{2 l}^{\prime}(z)}{2 l+6}
\end{aligned}
$$

After taking the curl of the vector potential to find the magnetic field components, we differentiate the field component $B_{x}$ with respect to the $y$-direction, and evaluate the terms along the $z$-axis. This function is then equated to the on-axis quadrupole gradient defined above (7.4). We find that

$$
a_{0}(z)=-\frac{g(z)}{2}
$$


The recursion relation generates the higher-order expansion coefficients,

$$
\begin{aligned}
a_{2}(z) & =\frac{g^{\prime \prime}(z)}{24}, \\
a_{4}(z) & =-\frac{g^{(i v)}(z)}{768}, \\
\text { etc. } &
\end{aligned}
$$

We will only be concerned with terms up to fourth-order. In terms of the quadrupole gradient function, $g(z)$, the quadrupole vector potential to fourth-order is given by

$$
\begin{aligned}
& A_{z}=\left(x^{2}-y^{2}\right)\left[-\frac{g}{2}+\frac{g^{\prime \prime}}{24}\left(x^{2}+y^{2}\right)-\frac{g^{(i v)}}{768}\left(x^{2}+y^{2}\right)^{2}\right], \\
& A_{x}=-\left[x^{3}-3 x y^{2}\right]\left[-\frac{g^{\prime}}{12}+\frac{g^{\prime \prime \prime}}{192}\left(x^{2}+y^{2}\right)\right], \\
& A_{y}=\left[y^{3}-3 x^{2} y\right]\left[-\frac{g^{\prime}}{12}+\frac{g^{\prime \prime \prime}}{192}\left(x^{2}+y^{2}\right)\right] .
\end{aligned}
$$

\section{Calculation of Field of REC Quadrupole}

The fields due to a rectangular block of permanent magnet material (ferrite, SmCo, $\mathrm{NdFeB}$ ) were derived and published by Marechal [81]. These expressions are analytical and exact for perfect blocks. Computing the fields from a quadrupole arrangement of rectangular blocks, such as are used in an RK-TBA system, we can determine the exact field at any point in the beampipe. While this is more information than we need, we may also use these expressions to calculate the exact on-axis quadrupole gradient function and its derivatives for use in (7.5).

\subsubsection{Solenoid}

The solenoid vector potential calculation proceeds very much like that of the quadrupole. In this case, however, there is only the azimuthal component of the vector potential, and it displays azimuthal symmetry. Following along the lines of the previous derivation we quickly determine the power series expansion

$$
\begin{aligned}
A_{\varphi}(r, z) & =r \sum_{l=0}^{\infty} a_{2 l}(z) r^{2 l}, \\
a_{2 l} & =-\frac{a_{2 l-2}^{\prime \prime}}{4 l(l+1)}, l \geq 1 .
\end{aligned}
$$


We apply the curl operator to the vector potential to obtain the axial magnetic field component. The lowest-order term in the power series is then identified with the on-axis magnetic field, $B_{z}(r=0, z)=B_{0}(z)=2 a_{0}(z)$.

To fourth-order in the expansion, the Cartesian components of the solenoid vector potential are

$$
\begin{aligned}
& A_{x}=-y\left[\frac{B_{0}}{2}-\frac{B_{0}^{\prime \prime}}{16}\left(x^{2}+y^{2}\right)\right], \\
& A_{y}=x\left[\frac{B_{0}}{2}-\frac{B_{0}^{\prime \prime}}{16}\left(x^{2}+y^{2}\right)\right] .
\end{aligned}
$$

\section{Calculation of on-axis solenoid field profile}

The on-axis field profile of a perfect solenoid is easily calculable from the field due to a current loop. Assuming the loop is circular with radius $a$, carries a current $I$, and is centered on and perpendicular to the $z$-axis, then the field at any point along the $z$-axis is given by [78]

$$
B_{z}(r=0, z)=\frac{\mu_{0}}{2} I \frac{a}{\left(a^{2}+z^{2}\right)^{3 / 2}} .
$$

A real solenoid will have a length $L$, a winding inner radius $R_{i n}$ and outer radius $R_{\text {out }}$. Assuming that the solenoid is centered with its longitudinal midpoint at $z=0$, and integrating over the length and transverse cross-section, the on-axis field can then be evaluated as

$$
\begin{aligned}
B_{z}(r=0, z) & =\left.\left.\frac{\mu_{0} J}{2}\left\{u \ln \left(r+\sqrt{u^{2}+r^{2}}\right)\right\}\right|_{r=R_{\text {in }}} ^{r=R_{\text {out }}}\right|_{u=z+L / 2} ^{u=z-L / 2}, \\
J & =\frac{I}{L\left(R_{\text {out }}-R_{\text {in }}\right)} .
\end{aligned}
$$

This expression is used to generate the coefficients in (7.6).

\subsection{Electrostatic Expansion}

The calculation of the vector potential proceeds along a slightly different route with the electrostatic components. First of all, we make the observation that electrostatic field problems are usually most easily solved analytically or numerically by using the scalar potential only. However, this only amounts to a change of gauge from that we are interested in obtaining. By implementing a change of gauge while respecting the Lorentz $U(1)$ symmetry group of the electromagnetic field, we are guaranteed invariance 
of the fields. More specifically, in the electrostatic case at hand, we first solve for the time-independent potentials $(\widetilde{\Phi}, \widetilde{\vec{A}})$ from the expressions for the fields

$$
\begin{aligned}
\vec{E}=-\vec{\nabla} \widetilde{\Phi}-\partial_{t} \widetilde{\vec{A}} & =-\vec{\nabla} \widetilde{\Phi} \\
\vec{B}=\vec{\nabla} \times \widetilde{\vec{A}} & =0 .
\end{aligned}
$$

We then introduce a pure gauge field $\Lambda(\vec{r}, t)$, and define new potentials via

$$
\begin{aligned}
\Phi & =\widetilde{\Phi}-\partial_{t} \Lambda \\
\vec{A} & =\widetilde{\vec{A}}+\vec{\nabla} \Lambda .
\end{aligned}
$$

We are at liberty to choose the form of the gauge field $\Lambda$. Making the choice

$$
\Lambda(\vec{r}, t)=\widetilde{\Phi}(\vec{r}) \cdot\left(t-t_{0}\right)
$$

the new potentials become

$$
\begin{aligned}
\Phi(\vec{r}, t) & =0 \\
\vec{A}(\vec{r}, t) & =\vec{\nabla} \widetilde{\Phi}(\vec{r}) \cdot\left(t-t_{0}\right) .
\end{aligned}
$$

Note that $\vec{A}$ remains curl-less, so that $\vec{B}=0$. This defines our program for finding the transverse gauge vector potential for electrostatic devices. The source-free region of the scalar potential satisfies Laplace's equation, so our previous method of solution is applicable here as well.

\section{Induction accelerating unit}

The geometry of an induction module exhibits azimuthal symmetry. This forces our interior region power series solution of Laplace's equation to take the form

$$
\begin{aligned}
\widetilde{\Phi}(r, z) & =\sum_{l=0}^{\infty} r^{2 l} \widetilde{a_{2 l}}(z), \\
\widetilde{a_{2 l}} & =-\frac{\widetilde{a}_{2 l-2}^{\prime \prime}}{4 l^{2}}, l \geq 1 .
\end{aligned}
$$


Performing the gauge transformation, the axial component vector potential is found to be

$$
\begin{aligned}
A_{z}(r, z) & =-\left(t-t_{0}\right) \sum_{l=0}^{\infty} r^{2 l} a_{2 l}(z), \\
a_{2 l} & =-\frac{a_{2 l-2}^{\prime \prime}}{4 l^{2}}, l \geq 1
\end{aligned}
$$

The series term coefficients are equated by $a_{2 l}(z)=-{\widetilde{a_{2 l}}}^{\prime}(z)$. The axial electric field component observed on-axis, $E_{z}(r=0, z)=E_{0}(z)$, is found to be equal to the lowestorder term in the series above, $a_{0}=E_{0}$. The radial component of the vector potential obeys the same symmetry rules as the axial component. The divergence-less condition of the vector potential in this new gauge and the solution to $A_{z}$ above, allows us to readily calculate the component $A_{r}$.

The deviation in arrival time of a particle from its fiducial must also be included in the final power series. If we denote the fiducial arrival time at location $z$ by $t_{g}$, and the deviation by $\hat{t}$, so that $t=t_{g}+\hat{t}$. To fourth-order, the induction module vector potential is then found to be

$$
\begin{aligned}
& A_{z}=-\left[\left(t_{g}-t_{0}\right)+\hat{t}\right]\left[a_{0}-\frac{a_{0}^{\prime \prime}}{4} r^{2}+\frac{a_{0}^{(i v)}}{64} r^{4}\right], \\
& A_{r}=\left[\left(t_{g}-t_{0}\right)+\hat{t}\right]\left[\frac{a_{0}^{\prime}}{2} r-\frac{a_{0}^{\prime \prime \prime}}{16} r^{3}\right] .
\end{aligned}
$$

In forming the gauge transformation of the scalar potential to the vector potential, we included an additional parameter $t_{0}$. This parameter is necessary as it fixes the lower limit of integration when we construct the vector potential from the electric field via

$$
\vec{E}=-\partial_{t} \vec{A}
$$

Specification of this parameter fixes the gauge by removing the remaining gauge degree of freedom. In practice, we will fix the value of $t_{0}$ to the arrival time of a given fiducial orbit at the entrance of the induction module gap.

\subsection{Standing Wave Electromagnetic Expansion}

To describe the electromagnetic potentials of standing wave rf cavities, we will follow a slightly different program than that used in describing the electrostatic induction 
modules. In this case, both electric and magnetic fields coexist. The fields are governed by a modal structure and, for each mode, the magnetic field may be described in terms of the electric field, and vice-versa. Hence, we will only consider the detailed description of the electric field of a given mode. Also, we only consider the TM modes of the cavity, since these will dominate the spectrum of modes excited from beam coupling. Each mode (labeled by the index $\lambda$ ) has associated with it a well-defined frequency $\left(\omega_{\lambda}\right)$ and amplitude, and exhibits the generic time-dependence

$$
\vec{E}(\vec{r}, t)=\vec{E}_{\lambda}(\vec{r}) \cos \left(\omega_{\lambda} t\right)
$$

Maxwell's equations then describe the spatial evolution of the source-free modal electric fields. For the electric fields this can be shown to be

$$
\begin{aligned}
\left(\nabla^{2}+k_{\lambda}^{2}\right) E_{z \lambda} & =0 \\
\left(\partial_{z z}+k_{\lambda}^{2}\right) \vec{E}_{\perp \lambda} & =\vec{\nabla}_{\perp} \partial_{z} E_{z \lambda}
\end{aligned}
$$

Here we are using the axial electric field of the mode as the primitive component, and from which the transverse components can be derived. All field components are realvalued functions.

The vector potential is defined by the usual relation in the transverse gauge for source-free potentials,

$$
\vec{E}=-\partial_{t} \vec{A}
$$

There are no free charges, so we may safely take the scalar potential to be zero everywhere. As in the electrostatic case, this definition contains an arbitrary integration constant. We remove this degree of freedom by specifying the lower limit of integration to correspond with the arrival time $\left(t_{0}\right)$ of the particle at the cavity entrance. In constructing a series of maps that span the cavity, the arrival time $t_{0}$ is taken at the beginning of the particular mapping interval. We also insert a reference phase $\left(\theta_{\lambda}\right)$ into the generic time-dependence, as calculated by the circuit equation which governs the beam-cavity coupling (see Chapter 9). The modal vector potential is then

$$
\begin{aligned}
\vec{A}_{\lambda}(\vec{r}, t) & =-\int_{t_{0}}^{t} d t^{\prime} \cos \left(\omega_{\lambda} t^{\prime}+\theta_{\lambda}\right) \vec{E}_{\lambda}(\vec{r}) \\
& =-\frac{1}{\omega_{\lambda}}\left[\sin \left(\omega_{\lambda} t+\theta_{\lambda}\right)-\sin \left(\omega_{\lambda} t_{0}+\theta_{\lambda}\right)\right] \vec{E}_{\lambda}(\vec{r})
\end{aligned}
$$

The electric field is used in this case to define the vector potential by explicit construc- 
tion. The program here is to solve the set (7.7) by the same techniques as previously described. We will find a connection of the resulting power series expansion to a given modal profile function, $E_{z \lambda}(\vec{r})$.

\subsubsection{Axial Electric Field Component}

We begin with the axial electric field component of the mode. We will assume that the mode has a specific azimuthal symmetry (indexed by $m$ ), found by performing a Fourier analysis in $\varphi$ on the entire electric field distribution. Performing the usual power series expansion in $r$, the axial component of a single-mode field can be described by

$$
E_{z \lambda}(\vec{r})=\cos m\left(\varphi-\varphi_{m}\right) \sum_{l=0}^{\infty} r^{m+l} a_{l}(z) .
$$

Inserting this into the axial component of (7.7), we find the recursion relation for the series expansion coefficients

$$
a_{2 l}=-\frac{a_{2 l-2}^{\prime \prime}+k_{\lambda}^{2} a_{2 l-2}}{2 l(2 l+2 m)}, l \geq 1,
$$

where the odd $l$ terms in the series are identically zero.

\subsubsection{Transverse Field Components}

Next, the transverse components of the mode are found. From the divergence-less criterion of the source-free modal electric field, we deduce some of the characteristics of these components:

- $E_{r \lambda}$ and $E_{z \lambda}$ have identical azimuthal dependence, while $E_{\varphi \lambda}$ experiences a $\pi / 2$ phase shift.

- The $m=0$ mode has no $E_{\varphi \lambda}$ component.

- $E_{r \lambda}$ and $E_{\varphi \lambda}$ have identical radial dependence, of one higher power than $E_{z \lambda}$.

Hence, the transverse mode components may be expressed in the forms

$$
\begin{aligned}
& E_{r \lambda}(\vec{r})=\cos m\left(\varphi-\varphi_{m}\right) \sum_{l=0}^{\infty} r^{m+l+1} b_{l}(z), \\
& E_{\varphi \lambda}(\vec{r})=\sin m\left(\varphi-\varphi_{m}\right) \sum_{l=0}^{\infty} r^{m+l+1} c_{l}(z) .
\end{aligned}
$$


Upon substitution of these forms into the transverse components of (7.7), we are able to deduce the relations between these series expansion coefficients and the coefficients of the axial field component (7.9). The modal electric field components to arbitrary order are then determined to be

$$
\begin{aligned}
& E_{z \lambda}(\vec{r})=\cos m\left(\varphi-\varphi_{m}\right) \sum_{l=0}^{\infty} r^{m+2 l} a_{2 l}(z) \\
& E_{r \lambda}(\vec{r})=-\cos m\left(\varphi-\varphi_{m}\right) \sum_{l=0}^{\infty} r^{m+2 l+1} \frac{(m+2 l) a_{2 l}^{\prime}}{4 l^{2}+2 l(2 l+2)+2 m} \\
& E_{\varphi \lambda}(\vec{r})=\sin m\left(\varphi-\varphi_{m}\right) \sum_{l=0}^{\infty} r^{m+2 l+1} \frac{(m) a_{2 l}^{\prime}}{4 l^{2}+2 l(2 l+2)+2 m}
\end{aligned}
$$

This effectively completes our program for determining the power series representation of the modal vector potential. Regardless of azimuthal symmetry, the axial field component is always present at lowest-order in the expansion series. We then choose the on-axis axial field component (or one of its transverse derivatives) to define the primitive function which generates the rest of the field.

\subsubsection{Vector Potential Expansion}

The vector potential is easily obtained by substituting the field components above (7.10) into the expression (7.8). The time-dependence must then be included in the net series expansion in powers of deviation coordinates. This is due to the use of the particle arrival time as a dynamical variable. The time-dependent phase seen by an arbitrary particle is given by (with $s=c t$ and $k_{\lambda}=\omega_{\lambda} / c$ )

$$
\begin{aligned}
\psi & =\omega_{\lambda} t+\theta_{\lambda} \\
& =k_{\lambda}\left(\widehat{s}+s_{g}\right)+\theta_{\lambda} \\
& =k_{\lambda} \widehat{s}+\left(k_{\lambda} s_{g}+\theta_{\lambda}\right) \\
& =\widehat{\psi}+\psi_{g}
\end{aligned}
$$

where we have distinguished the phase seen by the fiducial orbit $\left(\psi_{g}\right)$ from the phase difference $(\widehat{\psi})$ seen by a particle with a small deviation in arrival time. We denote by $\psi_{0}$ the phase at the initial time $t_{0}$. Expanding the bracketed expression in (7.8) to 
fourth-order,

$$
\begin{aligned}
\sin \psi-\sin \psi_{0} & =\sin \widehat{\psi} \cos \psi_{g}+\cos \widehat{\psi} \sin \psi_{g}-\sin \psi_{0} \\
& =\cos \psi_{g}\left[\widehat{\psi}-\frac{\widehat{\psi}^{3}}{3 !}\right]+\sin \psi_{g}\left[1-\frac{\widehat{\psi}^{2}}{2 !}+\frac{\widehat{\psi}^{4}}{4 !}\right]-\sin \psi_{0}
\end{aligned}
$$

This series is multiplied against the power series representing $\vec{E}_{\lambda}(\vec{r})$. We then retain only those terms up to fourth-order in all deviation coordinates. The factors $\cos \psi_{g}$, $\sin \psi_{g}, \sin \psi_{0}$ are to be regarded as constants.

\section{Monopole $(\mathbf{m}=\mathbf{0})$ mode}

This is the common mode in all idler and output rf cavities used in RK-TBA applications. For this mode, the on-axis static profile of the axial field component provides the primitive function. We denote by $E_{0}(z)$ the function $E_{z \lambda}(r=0, z)$. The expansion coefficients are then found to be

$$
\begin{aligned}
& a_{0}=E_{0}, \\
& a_{2}=-\frac{1}{4}\left(E_{0}^{\prime \prime}+k_{\lambda}^{2} E_{0}\right), \\
& a_{4}=\frac{1}{64}\left(E_{0}^{(i v)}+2 k_{\lambda}^{2} E_{0}^{\prime \prime}+k_{\lambda}^{4}\right) .
\end{aligned}
$$

Including time-dependent effects, the series expansion to fourth-order for the vector potential becomes

$$
\begin{aligned}
& A_{z}=-\frac{1}{\omega_{\lambda}}\left(a_{0}+a_{2} r^{2}+a_{4} r^{4}\right)\left(\cos \psi_{g}\left[\widehat{\psi}-\frac{\widehat{\psi}^{3}}{3 !}\right]+\sin \psi_{g}\left[1-\frac{\widehat{\psi}^{2}}{2 !}+\frac{\widehat{\psi}^{4}}{4 !}\right]-\sin \psi_{0}\right) \\
& A_{r}=\frac{1}{\omega_{\lambda}}\left(\frac{a_{0}^{\prime}}{2} r+\frac{a_{2}^{\prime}}{4} r^{3}\right)\left(\cos \psi_{g}\left[\widehat{\psi}-\frac{\widehat{\psi}^{3}}{3 !}\right]+\sin \psi_{g}\left[1-\frac{\widehat{\psi}^{2}}{2 !}+\frac{\widehat{\psi}^{4}}{4 !}\right]-\sin \psi_{0}\right) .
\end{aligned}
$$

We retain terms up to, and including, fourth-order only.

\section{Dipole $(\mathbf{m}=1)$ mode}

The dipole mode is the driven mode in the transverse chopper, and is the mode responsible for BBU effects in the induction cells and rf structures. It produces a transverse deflection of the beam, and is actively suppressed in the rf cavities in the main extraction section of an RK-TBA. However, it can not be entirely suppressed and its effect on the 
beam must be analyzed.

In this case, the axial electric field component is null on-axis, but exhibits a linear variation with distance from the axis. There are two independent polarizations for each dipole mode. Refering to (7.10), we will define the distance along the two polarization axes by

$$
\begin{aligned}
& x_{1}=r \cos \left(\varphi-\varphi_{1}\right), \\
& x_{2}=r \sin \left(\varphi-\varphi_{1}\right) .
\end{aligned}
$$

We can then define the primitive function for this mode as

$$
E_{1}(z)=\left.\partial_{x_{1}} E_{z \lambda}\right|_{r=0}(z)
$$

The series expansion coefficients are then

$$
\begin{aligned}
& a_{0}=E_{1} \\
& a_{2}=-\frac{1}{8}\left(E_{1}^{\prime \prime}+k_{\lambda}^{2} E_{1}\right) .
\end{aligned}
$$

And the vector potential follows as

$$
\begin{aligned}
& A_{z \lambda}=-\frac{1}{\omega_{\lambda}}\left(\cos \psi_{g}\left[\widehat{\psi}-\frac{\widehat{\psi}^{3}}{3 !}\right]+\sin \psi_{g}\left[1-\frac{\widehat{\psi}^{2}}{2 !}+\frac{\widehat{\psi}^{4}}{4 !}\right]-\sin \psi_{0}\right)\left(a_{0}+a_{2} r^{2}\right) r \cos \left(\varphi-\varphi_{1}\right), \\
& A_{r \lambda}=\frac{1}{\omega_{\lambda}}\left(\cos \psi_{g}\left[\widehat{\psi}-\frac{\widehat{\psi}^{3}}{3 !}\right]+\sin \psi_{g}\left[1-\frac{\widehat{\psi}^{2}}{2 !}+\frac{\widehat{\psi}^{4}}{4 !}\right]-\sin \psi_{0}\right)\left(\frac{a_{0}^{\prime}}{2} r+\frac{3}{14} a_{2}^{\prime} r^{3}\right) r \cos \left(\varphi-\varphi_{1}\right), \\
& A_{\varphi \lambda}=-\frac{1}{\omega_{\lambda}}\left(\cos \psi_{g}\left[\widehat{\psi}-\frac{\widehat{\psi}^{3}}{3 !}\right]+\sin \psi_{g}\left[1-\frac{\widehat{\psi}^{2}}{2 !}+\frac{\widehat{\psi}^{4}}{4 !}\right]-\sin \psi_{0}\right)\left(\frac{a_{0}^{\prime}}{2} r+\frac{1}{14} a_{2}^{\prime} r^{3}\right) r \sin \left(\varphi-\varphi_{1}\right) .
\end{aligned}
$$

Again, we keep only those terms up to fourth-order.

\subsection{Constant-z Versus Constant-t Representations}

As discussed in Chapter 10, there are situations in which we are interested in a representation of the field distribtution of a beamline element at a given moment in time. This is the constant- $t$ representation of the field instead of the constant- $z$ representation discussed up to now. In the constant-z representation we were concerned with the field at a given beamline location and discussed changes in the observed field due to variations in the particle arrival time. In the constant-t representation, we will freeze the fields at a given time (again defined by a fiducial orbit) and expand the field distribution in 
a power series in $z$ about the position of the fiducial orbit location. Since we already have primitive functions defined as a function of $z$ it is a relatively straightforward job to produce a Taylor series by analytical or numerical differentiation. This series is combined with the transverse power series expansion in the same manner in which the arrival time deviation expansion was handle in the previous section on rf cavity modes. The combined series is terminated at fourth-order in all deviation variables. 


\section{Chapter 8}

\section{Self-Field Impulses}

\subsection{Introduction}

This chapter provides an overview and description of the theoretical formalism and computational methods employed in calculating the effects on beam dynamics due to the distribution of charge and current convected along by the beam itself

The algorithms described here for generating fields and impulses at the location of the macroparticles themselves differ from our earlier approach that treats external fields and their forces from a Hamiltonian expansion, although they are equivalent. In a nonself-consistent description of the beam dynamics, the beam distribution and its self-fields may be approximately described by a fitting procedure to an analytic functional form. In this case, the self-field effects may be incorporated within our original Hamiltonian framework. But, by postulating a fixed form for the beam distribution ensures its selfsimilar evolution. To accurately determine the influence of self-fields upon the trajectory of individual particles, and upon the evolution of the beam distribution, however, requires a description of these fields with much greater resolution than is necessary to describe the fields from external beamline elements. A functional approximation even at the highest polynomial order present in the external field description prevents the study of the evolution of the beam distribution at the local scale, and can not compute the deterioration of beam quality from self-consistent, self-field effects. For beams in the devices under consideration in this dissertation, we wish to study the evolution of the detailed beam distribution, and the algorithms described here present a highly efficient method for doing so. 


\subsection{Brief Description of Particle-in-Cell Algorithms}

PIC algorithms were first developed in the 1960's by Buneman and Dawson to study kinetic effects in warm plasmas with a computer simulation. The field has been developed further by many others, most notably Birdsall (U.C. Berkeley) and Langdon (LLNL), and Hockney and Eastwood (Reading University, UK). The history of this field is contained within the two primary texts and references of the field [82] [83]. A PIC simulation seeks to study the self-consistent evolution of an ensemble of particles with external and selfelectromagnetic fields. This can be done with a relatively small number of simulation macroparticles $\left(10^{3}-10^{6}\right.$, depending upon the dimensionality and desired resolution) compared to the actual number of particles in a real plasma $\left(10^{14}-10^{21}\right.$, for typical laboratory and astrophysical systems).

A PIC simulation begins by construction of a grid or mesh system to describe the physical boundaries and internal geometry where the plasma exists. Once this mesh is constructed, the generic simulation proceeds through a repeating series of steps:

1. Charge assignment - the individual macroparticles are assigned to grid nodes, either wholly or partially, according to some prescription that maintains total charge conservation. There is usually an order of magnitude more particles than grid nodes, to prevent spurious or noise effects from having a significant contribution.

2. Solution of the electrostatic (or electromagnetic) potential(s) on the grid. This job can be efficiently and quickly dispatched by use of a so-called rapid elliptic solver of the underlying Poisson or Helmholtz equations.

3. Field components are computed on the grid by finite differences and then interpolated to the actual position of the macroparticles. This interpolation procedure is the dual process to charge assignment, and both are determined during the same prescription. This may be a zeroth order (NGP, or 'nearest grid point'), first order (PIC and CIC, 'cloud-in-cell'), or higher ordr process.

4. Impulses are derived from the fields sampled by each particle, and their coordinates are advanced in time.

\subsection{Lorentz Force and Kick Mapping Approximation}

The effects of self-fields are introduced into the beam dynamics calculations in an approximate way. Here, we assume that time step in the mapping interval is small enough 
that the individual particles do not change their relative positions much. That is, the distribution of particles in configuration space remains essentially constant over the small time interval and, hence, the self-fields remain unchanged. This permits us to calculate the force on an individual particle and know that that force itself remains essentially constant over the time interval. The impulse derived from this constant force is then simple to evaluate. The kick mapping approximation is then that the changes to the particle coordinates over the interval occur only in the momenta.

\section{Lorentz Force on Particles}

The force experienced by individual particles in a given inertial reference frame is given by the Lorentz force law. In MKS units,

$$
\vec{F}=q(\vec{E}+\vec{v} \times \vec{B})
$$

We will typically consider two distinct reference frames: the laboratory frame $(l)$ and the beam's center of momentum (CM) frame $(b)$. For calculation of the self-fields we will work in the beam frame. But, for calculating the resulting impulse we will require expressions for the fields and the Lorentz force in the laboratory frame.

In this discussion we will assume a beam that possesses a much larger longitudinal momentum component than either transverse component. Here, we also assume that the energy spread of the particles within the beam is small enough that only non-relativistic motion is present in the beam's CM frame. If these two conditions hold, then the selffields in the $b$ frame are primarily electrostatic in origin, and are due to the detailed distribution of charges within the beam, while the current density distribution is to be ignored. Hence, the electric field components of the Lorentz force dominate the magnetic field contributions. We will make the approximation then,

$$
\overrightarrow{B^{b}}=0 \text {. }
$$

The field components in the $b$ frame must then be Lorentz transformed to the electric and magnetic field components in the $l$ frame via:

$$
\begin{aligned}
E_{x}^{l}=\gamma E_{x}^{b}, & B_{x}^{l}=-\frac{1}{c} \gamma \beta E_{y}^{b}, \\
E_{y}^{l}=\gamma E_{y}^{b}, & B_{y}^{l}=\frac{1}{c} \gamma \beta E_{x}^{b}, \\
E_{z}^{l}=E_{z}^{b}, & B_{z}^{l}=0,
\end{aligned}
$$


where $\gamma$ and $\beta$ are the usual parameters for the Lorentz boost between the two frames.

The force components in the $l$ frame are then found to be

$$
\begin{aligned}
F_{x}^{l}=q\left(\gamma E_{x}^{b}-\beta^{2} \gamma E_{x}^{b}\right) & =\frac{q}{\gamma} E_{x}^{b}, \\
F_{y}^{l}=q\left(\gamma E_{y}^{b}-\beta^{2} \gamma E_{y}^{b}\right) & =\frac{q}{\gamma} E_{y}^{b}, \\
F_{z}^{l}=q E_{z}^{l} & =q E_{z}^{b} .
\end{aligned}
$$

\section{Impulse Mapping}

The Lorentz force is just the change in the mechanical momentum of the particle with respect to time. That is,

$$
\vec{F}=\frac{d \vec{p}}{d t}=\frac{d(\gamma \vec{\beta} m c)}{d t} .
$$

To connect with our description of single particle motion where we defined various normalized momenta and fields, we will use the normalization

$$
\begin{aligned}
\vec{f} & =\vec{F} / m c \\
\widetilde{E_{i}^{b}} & =\frac{\varepsilon_{0}}{Q} E_{i}^{b},
\end{aligned}
$$

where $Q$ is the total charge confined to the computational box. We are interested in the changes to the variables $\left(\gamma \beta_{x}\right),\left(\gamma \beta_{y}\right)$, and $(\gamma)$. From the normalized Lorentz force,

$$
\begin{aligned}
\frac{d}{d t}\left(\gamma \beta_{x}\right)=f_{x} & =\frac{q Q}{\gamma m c \varepsilon_{0}} \widetilde{E_{x}^{b}}, \\
\frac{d}{d t}\left(\gamma \beta_{y}\right)=f_{y} & =\frac{q Q}{\gamma m c \varepsilon_{0}} \widetilde{E_{y}^{b}}, \\
\frac{d}{d t}(\gamma)=\vec{f} \cdot \vec{\beta} & =\frac{q Q}{\gamma m c \varepsilon_{0}}\left(\beta_{x} \widetilde{E_{x}^{b}}+\beta_{y} \widetilde{E_{y}^{b}}+\gamma \beta_{z} \widetilde{E_{z}^{b}}\right) .
\end{aligned}
$$

This force occurs over a well-defined time interval $(\Delta t)$. The generated impulses to the canonical variables over this interval are then approximated by

$$
\begin{aligned}
\Delta\left(\widehat{p_{x}}-\widehat{A_{x}}\right) & =\Delta\left(\gamma \beta_{x}\right) \cong \frac{d}{d t}\left(\gamma \beta_{x}\right) \cdot \Delta t, \\
\Delta\left(\widehat{p_{x}}-\widehat{A_{x}}\right) & =\Delta\left(\gamma \beta_{x}\right) \cong \frac{d}{d t}\left(\gamma \beta_{x}\right) \cdot \Delta t, \\
\Delta(-\gamma) & \cong \quad-\frac{d}{d t}(\gamma) \cdot \Delta t,
\end{aligned}
$$


with the expressions for the forces given above. The calculation of the self-field mapping is now complete once we have the values of the electric field at the location of the particles in the beam's CM frame, to which we now turn.

\subsection{Formulation in Terms of Potentials}

We are looking for a framework to calculate self-fields of a finite charge and current distribution in the presence of a bounding, conducting tube. From Maxwell's equations,

$$
\begin{aligned}
\vec{\nabla} \times \vec{E} & =-\partial_{t} \vec{B}, \\
\vec{\nabla} \times \vec{H} & =\partial_{t} \vec{D}+\vec{J}, \\
\vec{\nabla} \cdot \vec{D} & =\rho, \\
\vec{\nabla} \cdot \vec{B} & =0,
\end{aligned}
$$

and the equation of continuity

$$
\vec{\nabla} \cdot \vec{J}+\partial_{t} \rho=0
$$

we merely need specify the charge $(\rho)$ and current $(J)$ distributions within some physical volume, with appropriate boundary conditions, to have a mathematically well-defined problem. In particular, we can look for a set of scalar and vector potentials from which to derive our electric and magnetic fields via:

$$
\begin{aligned}
\vec{E} & =-\partial_{t} \vec{A}-\vec{\nabla} \Phi, \\
\vec{B} & =\vec{\nabla} \times \vec{A},
\end{aligned}
$$

where the potentials are constrained by the gauge condition:

$$
\vec{\nabla} \cdot \vec{A}+\frac{\xi}{c^{2}} \partial_{t} \Phi=0
$$

Here, $\xi$ is an arbitrary parameter which sets the gauge consistent with the $\mathrm{U}(1)$ symmetry of the fields. In this formulation, Maxwell's equations are expressed in terms of the potentials by

$$
\nabla^{2} \vec{A}-\frac{1}{c^{2}} \partial_{t t} \vec{A}+\left(\frac{1}{\xi}-1\right) \vec{\nabla}(\vec{\nabla} \cdot \vec{A})+\mu \vec{J}=0
$$




$$
\nabla^{2} \Phi-\frac{\xi}{c^{2}} \partial_{t t} \Phi+\frac{\rho}{\varepsilon}=0
$$

We will choose to work within the Lorentz gauge $(\xi=1)^{1}$. In the laboratory reference frame, the longitudinal current is much greater than any transverse current component (ie., $J_{z} \gg J_{\perp}$ ), and hence the transverse components of the vector potential are neglegible compared to the longitudinal component. Hence, Maxwell's equations in potential form reduce to

$$
\begin{aligned}
\nabla^{2} A_{z}-\frac{1}{c^{2}} \partial_{t t} A_{z}+\mu J_{z} & =0 \\
\nabla^{2} \Phi-\frac{1}{c^{2}} \partial_{t t} \Phi+\frac{\rho}{\varepsilon} & =0
\end{aligned}
$$

with the gauge condition

$$
\partial_{z} A_{z}+\frac{1}{c^{2}} \partial_{t} \Phi=0
$$

and the continuity relation

$$
\partial_{z} J_{z}+\partial_{t} \rho=0
$$

The calculation of the fields is performed with a finite volume defined by the beam pipe in the transverse (xy) directions and either (i) the entire extent of the beam distribution in the z-direction, or (ii) a single longitudinal spatial period (at the fundamental frequency) of a modulated beam. In both cases, Fourier analysis and synthesis techniques may be employed along the longitudinal direction. The finite computational volume encloses a finite, well-defined quantity of electric charge, $Q$. We will normalize the charge and current distributions to this value by defining,

$$
\begin{aligned}
n(\vec{r}) & =\frac{\rho(\vec{r})}{Q}, \\
\beta_{z}(\vec{r}) n(\vec{r}) & =\frac{J_{z}(\vec{r})}{Q},
\end{aligned}
$$

such that

$$
\int_{\text {vol. }} n(\vec{r}) d^{3} r=1
$$

This also induces a natural normalization to the potentials. We define

$$
\widetilde{A_{z}}=\frac{A_{z}}{Q Z_{0}}
$$

\footnotetext{
${ }^{1}$ Other gauges correspond to different values of $\xi$. The Coulomb gauge, for example, is chosen by setting $\xi=0$.
} 


$$
\widetilde{\Phi}=\frac{\varepsilon_{0} \Phi}{Q}
$$

where $Z_{0}=\sqrt{\mu_{0} / \varepsilon_{0}}$ is the impedance of free space. With this normalization, our system of equations become

$$
\begin{gathered}
\nabla^{2} \widetilde{A_{z}}-\frac{1}{c^{2}} \partial_{t t} \widetilde{A_{z}}+\beta_{z} n=0, \\
\nabla^{2} \widetilde{\Phi}-\frac{1}{c^{2}} \partial_{t t} \widetilde{\Phi}+n=0, \\
\partial_{z} \widetilde{A_{z}}+\partial_{t} \widetilde{\Phi}=0 \\
\partial_{z}\left(\beta_{z} n\right)+\partial_{t} n=0 .
\end{gathered}
$$

The d'Alembertian operator, $\nabla^{2}-\frac{1}{c^{2}} \partial_{t t}$, is expressable as $\nabla_{\perp}^{2}+\partial_{z z}-\frac{1}{c^{2}} \partial_{t t}$, which clearly separates the transverse, longitudinal, and temporal derivatives.

We will make one more main approximation, which will differentiate our approach from that of typical electromagnetic particle-in-cell simulations, and that is to neglect high-frequency wave propagation (ie. wavelengths much smaller than the dimensions of the computational box) while retaining the low-frequency portion of the spectrum. This is consistent with our overall philosophy and approach via mapping. The fields and potentials are derived from a given distribution of the beam's particles at a given instant in time. The impulses given to the particles are assumed to be constant over the duration during which the mapping is applied. Thus, the beam distribution is assumed to be static in time (at least, over this short duration) and hence so will be the potentials and fields. In this case, we neglect the time dependence in the field equations of motion, obtaining

$$
\begin{aligned}
\nabla_{\perp}^{2} \widetilde{A_{z}}+\partial_{z z} \widetilde{A_{z}}+\beta_{z} n & =0 \\
\nabla_{\perp}^{2} \widetilde{\Phi}+\partial_{z z} \widetilde{\Phi}+n & =0
\end{aligned}
$$

In this case, our choice of gauge is indistinguishable from the Coulomb gauge.

\section{Transformation to the Beam Frame}

In the beam frame, we follow our earlier approximation and neglect the current sources to the field compared with the charge sources. We then neglect the vector potential in favor of the scalar potential only, since this is allowed by our choice of gauge. A Lorentz 
transformation connects the beam frame to the laboratory frame, and maintains the form of the field equations by general covariance. In the previous section, we have shown the Lorentz force at the locations of the particles in the laboratory frame due to the field components calculated in the beam frame. Our program for calculating selffield impulses is now clear. Starting with a distribution of particles in the laboratory frame, a Lorentz transformation takes this to a distribution in the beam frame. A single scalar potential function is then found in this transformed computational region, and the electric field components are calculated at the location of the particles. Finally, the field components are then used to determine the impulses in the laboratory frame.

\subsection{Calculation of Potentials}

The field equations for the potentials exhibit a useful symmetry in their form. They are both 3D Helmholtz equations with similar source terms. At the conducting boundaries, the parallel components of the electric field are identically zero. Hence, the values of the potentials, $A_{z}$ and $\Phi$, at these boundaries are both constant (which will be taken to be zero). Even though the source terms exhibit small differences, the field equations and boundary conditions are the same. We may then use identical techniques to find solutions for both. We are left with the task of identifying and utilizing techniques to numerically solve the Helmholtz equation in the computational volume.

The Helmholtz equation belongs to the larger class of elliptic partial differential equations. These equations have been extensively studied by numerical analysts, and many fast, accurate, and compact algorithms have been developed to solve them. Currently, the multigrid algorithm [84] is receiving much attention, and has been shown to outperform many of its predecessors in speed and accuracy. A thorough, though dated, overview of field solvers used in particle simulations is given by Hockney and Eastwood [83]. Smith [85] gives a good introduction to numerical methods for solving second order partial differential equations, and was found to be invaluable for assistance in rendering the algorithms to accommodate non-conformal boundaries ${ }^{2}$.

We will always work with a Cartesian mesh in the xy-plane. This considerably simplifies the numerical analysis and solution of the Helmholtz equation since the transverse Laplacian operator takes on an uncoupled and symmetric form. Of course, we must then sacrifice simplicity in the description of the curved beampipes. In general,

\footnotetext{
${ }^{2}$ A non-conformal boundary is one that may only be approximately fit by the underlying mesh geometry - eg. a curved boundary surface fit to an evenly spaced, Cartesian grid.
} 
the cross-section of the conducting boundary may take an arbitrary form and may vary along the longitudinal direction. Without sacrificing much of the detailed description of the beamline environment, we will assume the cross-section to be elliptical in xy, and invariant along z. This allows us to use the conformal symmetry of the Helmholtz equation to independently scale distances in the $\mathrm{x}$ and $\mathrm{y}$ directions without altering the form of the equation and the algorithm for its solution. Henceforth, we will only discuss the numerical methods of solving the Helmholtz equations on a square grid in the xy plane with a circular boundary centered on the grid.

The multigrid solver we employ has been optimized to find solutions inside a circular boundary of unit radius. The transverse coordinates are then normalized by the physical pipe radius $(R)$,

$$
\begin{aligned}
& u=x / R, \\
& v=y / R .
\end{aligned}
$$

The transverse Laplacian operator is then

$$
\nabla_{\perp}^{2}=\partial_{x x}+\partial_{y y}=\frac{1}{R^{2}}\left(\partial_{u u}+\partial_{v v}\right)
$$

\section{Discrete Fourier Analysis}

The physical environment we are considering is such that the boundaries are assumed to have an infinite extent along the z-axis, and to be invariant with respect to longitudinal position. The source terms are either periodically spaced microbunches, or isolated bunches $^{3}$. We perform a discrete Fourier decomposition of the source terms along the longitudinal direction. Letting $L$ denote the period length of the volume, and allowing the particles to be distributed over the interval $[0, L]$, we normalize the z-coordinates by $2 \pi / L$ by defining $\zeta=\frac{2 \pi}{L} z$. The Laplacian operator is then

$$
\nabla_{\perp}^{2}+\partial_{z z}=\nabla_{\perp}^{2}+\left(\frac{2 \pi}{L}\right)^{2} \partial_{\zeta \zeta}
$$

We discretize the longitudinal interval into $N$ discrete nodes. The $1 \mathrm{D}$ discrete Fourier transform (DFT), $\widehat{f}_{l}$, for a function, $f(z)$, defined on this interval by its values at the

\footnotetext{
${ }^{3}$ In the case of a single, isolated bunch the longitudinal extent of the computational volume used in the field solver is taken to be twice that of the bunch. An approximation is then made to apply periodic boundary conditions to the potential at the longitudinal boundaries.
} 
nodes, $f\left(z_{j}\right) \rightarrow f_{j}=f\left(\zeta_{j}\right)$, is given by

$$
\widehat{f}_{l}=\frac{2 \pi}{N} \sum_{j=1}^{N} f_{j} \exp [-2 \pi i(j-1)(l-1) / N],
$$

with inverse given by

$$
f_{j}=\frac{1}{2 \pi} \sum_{l=1}^{N} \widehat{f}_{l} \exp [2 \pi i(j-1)(l-1) / N] .
$$

Using a second order finite differencing scheme, the differential operator $\partial_{\zeta \zeta}$ is discretized,

$$
\partial_{\zeta \zeta} f_{j} \rightarrow \frac{1}{\delta^{2}}\left(f_{j+1}-2 f_{j}+f_{j+1}\right)
$$

where

$$
\delta=\frac{2 \pi}{N}
$$

In the DFT basis, this operator becomes

$$
\begin{aligned}
\partial_{\zeta \zeta} f_{j} & \rightarrow\left(\frac{N}{2 \pi}\right)^{2}\left(f_{j+1}-2 f_{j}+f_{j+1}\right) \\
& =\left(\frac{N}{2 \pi}\right)^{2} \frac{1}{2 \pi} \sum_{l=1}^{N} \widehat{f}_{l} \exp [2 \pi i(j-1)(l-1) / N]\{\exp [2 \pi i(l-1) / N]-2+\exp [-2 \pi i(l-1) / N]\} \\
& =\left(\frac{N}{2 \pi}\right)^{2} \frac{1}{2 \pi} \sum_{l=1}^{N} 2\left(\cos \left[\frac{2 \pi(l-1)}{N}\right]-1\right) \widehat{f}_{l} \exp [2 \pi i(j-1)(l-1) / N]
\end{aligned}
$$

We perform a DFT expansion of the scalar potential $\left(\widetilde{\Phi}_{j} \rightarrow \widehat{\Phi}_{l}\right)$ and the source terms $\left(n_{j} \rightarrow \widehat{n}_{l}\right)$. Our original 3D Helmholtz equation is then transformed into a set of $2 N 2 \mathrm{D}$ Helmholtz equations in the DFT basis, with the $l^{t h}$ member of this set being

$$
\nabla_{\perp}^{2} \widehat{\Phi}_{l}+\left(\frac{N}{L}\right)^{2} 2\left(\cos \left[\frac{2 \pi(l-1)}{N}\right]-1\right) \widehat{\Phi}_{l}+\widehat{n}_{l}=0
$$

and with the transformed sources and fields now taking on complex values. We can express this more efficiently and compactly as

$$
\begin{gathered}
\left(\partial_{u u}+\partial_{v v}+\Gamma_{l}^{2}\right) \widehat{\Phi}_{l}+R^{2} \widehat{n}_{l}=0 \\
\Gamma_{l}^{2}=2\left(\frac{N R}{L}\right)^{2}\left(\cos \left[\frac{2 \pi(l-1)}{N}\right]-1\right) .
\end{gathered}
$$


These complex-valued, 2D Helmholtz equations are solved numerically by an elliptic solver for the individual DFT basis components of the potential, $\widehat{\Phi}_{l}$. An inverse DFT transformation then restores the potential values on the grid nodes, $\Phi_{j}$. Since we now have the potential values on a $3 \mathrm{D}$ grid, the electric field components at the grid nodes are rapidly computed by discrete differentiation. The field components at the particles are then interpolated from the values at the surrounding grid points.

\section{Multigrid Algorithm}

The traditional methods for solving elliptic boundary value problems numerically have involved Fourier analysis, iterative cyclic reduction, and/or relaxation methods. The two former classes of techniques, known collectively as 'rapid' elliptic solvers, when applied to a mesh with $\mathrm{N}$ grid points, typically converge in $O(\mathrm{~N} \log \mathrm{N})$ operations. The multigrid methods [84] [86], based upon relaxation algorithms, can converge in $O(\mathrm{~N})$ operations. Additionally, multigrid algorithms can solve more general elliptic equations with more general geometries and matching conditions at the boundaries. Multigrid methods are faster, more generally applicable, and have become the method of choice for solving most elliptic equations.

In the first incarnation of the multigrid method a single, fine mesh is generated and source terms are evaluated upon the grid points. Coarser grids are generated and used as an aid to speed convergence of a specific relaxation technique. The current implementation requires that the source terms be evaluated at the mesh points on the coarser as well as the finer grids. Solutions are obtained at progressively finer grids, until a final, pre-specified mesh fineness is reached.

The multigrid methods succeed due to the complementary error-reduction capabilities of relaxation and coarse-grid correction. Relaxation methods are very good at smoothing, and hence in reducing high-frequency error components in a small number of iterations. But, they require many times more iterations to decrease the low-frequency (smooth) error components. However, by introducing a coarser grid, these previously low-frequency components become high-frequency (with respect to the grid), and are rapidly dispatched. Thus, by cycling between coarse and fine grids, resolution at the finest meshing is maintained while both high- and low-frequency errors are rapidly reduced. Multigrid methods are so called because they tend to use three or more different meshes. A solution 'cycle' will begin at the finest mesh, becoming coarser and coarser until there only remains a single mesh point. The finite difference equations can then be solved exactly. The grid is then refined until the initial resolution is reached. This 
cycle, or a slight variant, is then repeated until convergence of the solution is reached. This can require a mere one or two cycles only.

\subsection{Comparison with Theoretical Models}

A direct comparison can be made between the potentials and fields calculated by this algorithm with a distinct theoretical model. Here, consider only those beam distributions that are uniform in the longitudinal direction, symmetric in azimuthal angle, and centered in the beampipe. This permits an analytic solution for the potentials for a variety of charge distributions.

The electrostatic potential satisfies Poisson's equation

$$
\nabla^{2} \Phi=-\rho(\vec{r}) / \epsilon_{0}
$$

With uniformity in $\mathrm{z}$ and $\phi$, this PDE becomes

$$
\frac{1}{r} \partial_{r}\left(r \partial_{r} \Phi\right)=-\rho(r) / \epsilon_{0}
$$

This equation is easily solved for a variety of transverse charge density distributions, using standard techniques. The boundary condition at the beampipe wall (radius $b$ ) is taken to be $\Phi(b)=0$.

The example is the standard KV distribution [87]. This is a distribution in the 4D transverse phase space, which has been exceedingly useful for analytic calculations of beam envelope dynamics with self-field effects included. The normalized phase space distribution (ie. total charge, $\mathrm{Q}=1$ ) is given by

$$
f\left(r_{4}\right)=\frac{1}{2 \pi^{2} a^{3}} \delta\left(r_{4}-a\right) .
$$

Here, $r_{4}$ is a radial measure in the $4 \mathrm{D}$ phase space. The distribution is singular in the $4 \mathrm{D}$ phase space, but produces a uniform distribution in any $2 \mathrm{D}$ projection. Shown in Figure 8.1 is the projection in the xy plane. The round beampipe is located at a radius of $7 \mathrm{~mm}$. The particle density, $n(r)=\rho(r) / Q_{\text {total }}$, in this space is given by

$$
n(r)=n_{0}=\frac{\rho_{0}}{Q_{\text {total }}}=\frac{1}{\pi a^{2} L},
$$

where $a$ is the edge radius ( $4.8 \mathrm{~mm}$ in the Figure) and $b$ the radius of the beampipe 


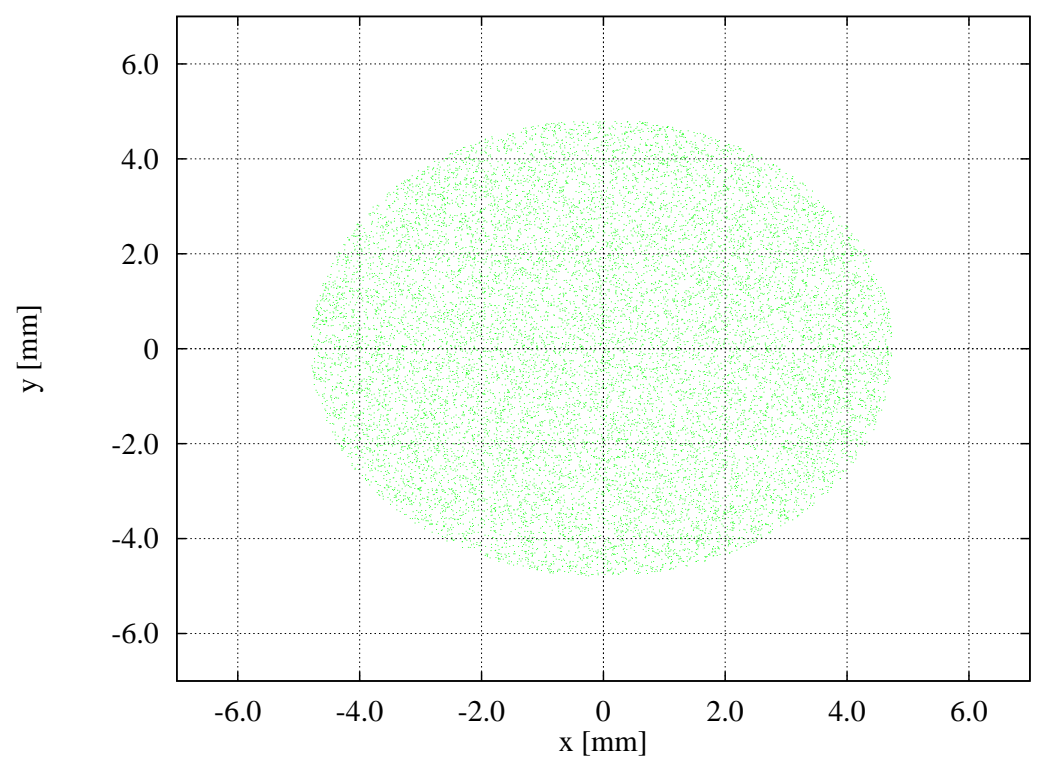

Figure 8.1: Loading of particles in the xy plane for a KV distribution.

$(7 \mathrm{~mm})$. The bunch length is finite, taken to be the wavelength of the rf modulation frequency in the simulation $(2.624 \mathrm{~cm})$, and in the rest frame of the beam $L=\gamma \lambda$. For a $4.0 \mathrm{MV}$ beam $(\gamma=5.892), L=0.232 \mathrm{~m}$. Hence, the uniform particle density takes the value $n_{0} \simeq 8.9 \cdot 10^{4}$.

The solution to equation (8.4) for the scalar potential is

$$
\begin{aligned}
& \Phi(0 \leq r \leq a)=\frac{\rho_{0} / \epsilon_{0}}{4} a^{2}\left(1+2 \ln \frac{b}{a}\right)-\frac{\rho_{0} / \epsilon_{0}}{4} r^{2}, \\
& \Phi(a \leq r \leq b)=\frac{\rho_{0} / \epsilon_{0}}{2} a^{2} \ln \frac{b}{r} .
\end{aligned}
$$

The electric field components are easily derived, $E_{\phi}=0$ and $E_{r}=-\partial_{r} \Phi$ or

$$
\begin{aligned}
& E_{r}(0 \leq r \leq a)=\frac{\rho_{0} / \epsilon_{0}}{2} r, \\
& E_{r}(a \leq r \leq b)=\frac{\rho_{0} / \epsilon_{0}}{2} \frac{a^{2}}{r} .
\end{aligned}
$$

We impose the normalization condition (8.3) to obtain the normalized potentials and fields

$$
\widetilde{\Phi}(0 \leq r \leq a)=\frac{1}{4 \pi L}\left(1+2 \ln \frac{b}{a}\right)-\frac{1}{4 \pi a^{2} L} r^{2},
$$




$$
\begin{aligned}
\widetilde{\Phi}(a \leq r \leq b) & =\frac{1}{2 \pi L} \ln \frac{b}{r} . \\
\widetilde{E}_{r}(0 \leq r \leq a) & =\frac{1}{2 \pi a^{2} L} r, \\
\widetilde{E}_{r}(a \leq r \leq b) & =\frac{1}{2 \pi L} \frac{1}{r} .
\end{aligned}
$$

The particle density distribution and normalized potential are shown in Figure 8.2. The analytic solution for the scalar potential at $\mathrm{x}=\mathrm{y}=0$ is $\widetilde{\Phi}=0.903 \mathrm{~m}^{-1}$. In this simulation 16384 particles were loaded onto a $33 \times 33 \times 16$ node mesh. However, since the particle loading was performed over a $6 \mathrm{D}$ phase space, small nonuniformities in the $4 \mathrm{D}$ projection, and subsequently in the $2 \mathrm{D}$ projections are inevitable. This is most easily seen in the plot of the normalized particle density, where $\pm 10 \%$ variations in the flat-top portion of the distribution exits. However, this noise is corrected for in the multigrid algorithm via smoothing. The scalar potential reaches a peak of value of $0.908 \mathrm{~m}^{-1}$, a difference of $0.5 \%$ from the theoretical value.

The electric field components are shown in Figures 8.3 and 8.4. The radial electric field is linear in the interior region of the charge distribution, reaching a theoretical maximum of $214 \mathrm{~m}^{-2}$, and then it follow an inverse-r decay to a value of $147 \mathrm{~m}^{-2}$ at the beam pipe wall. The simulation yields values of approximately $203 \mathrm{~m}^{-2}$ and $150 \mathrm{~m}^{-2}$, respectively. The fields thus display an error of a few per cent. However, the degree of linearity and smoothness in the interior region of the beam is impressive. The lack of any significant noise is an improvement over other numerical methods. Subsequently, we are assured that the multigrid methods are suitable for beam tracking experiments in that they will not induce excessive amounts of beam heating and emittance growth from noise sources. 


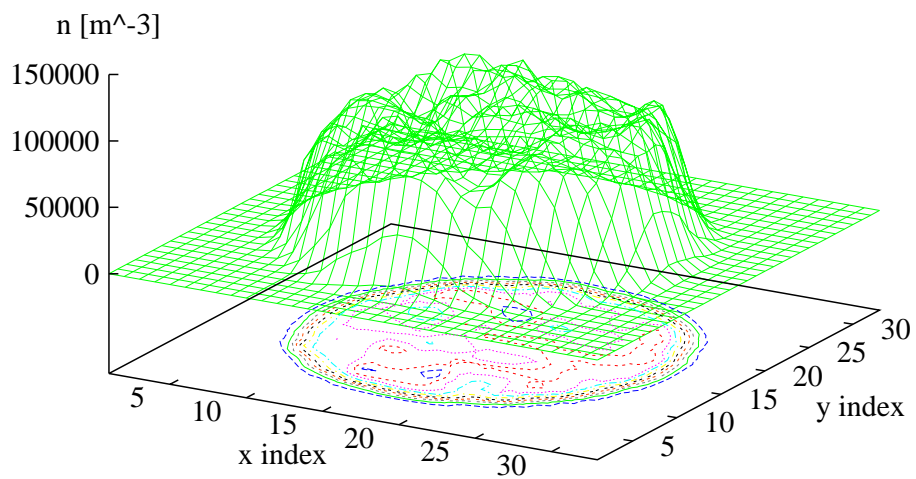

(a)

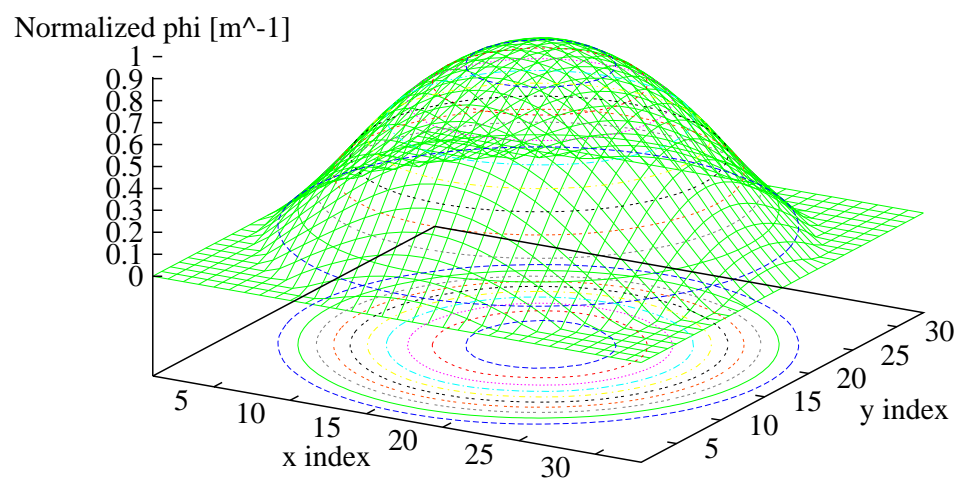

(b)

Figure 8.2: Normalized charge density (a) and electrostatic potential (b) of the KV distribution. 


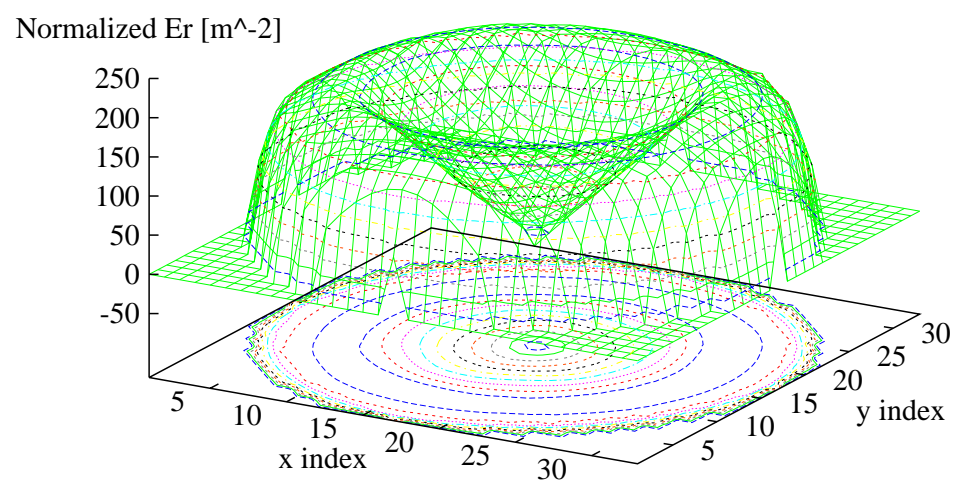

(a)

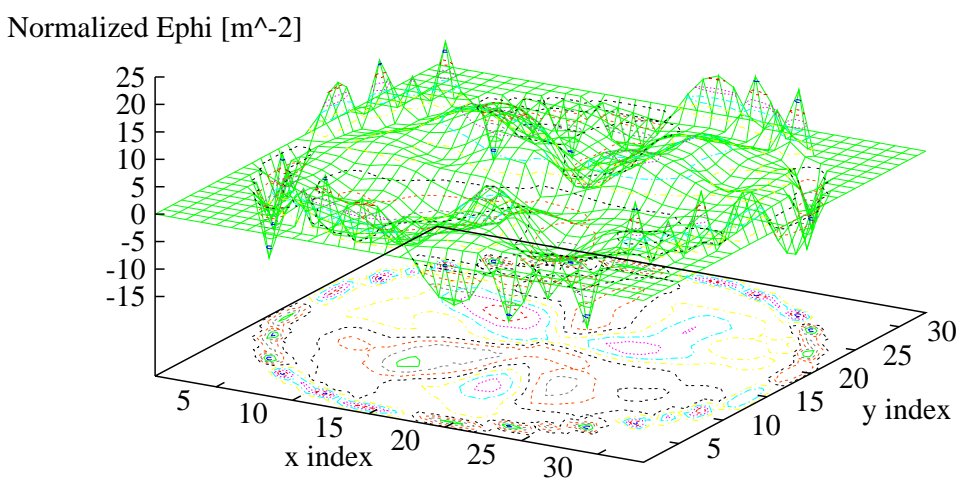

(b)

Figure 8.3: (a) Radial- and (b) azimuthal- electric field components of the KV distribution. 


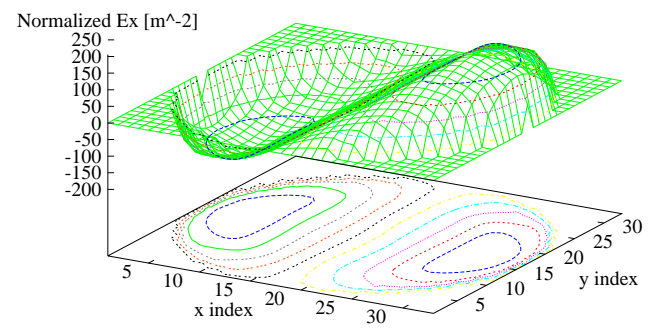

(a)

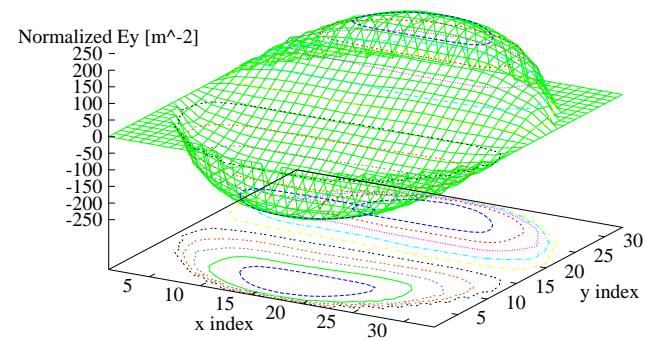

(b)

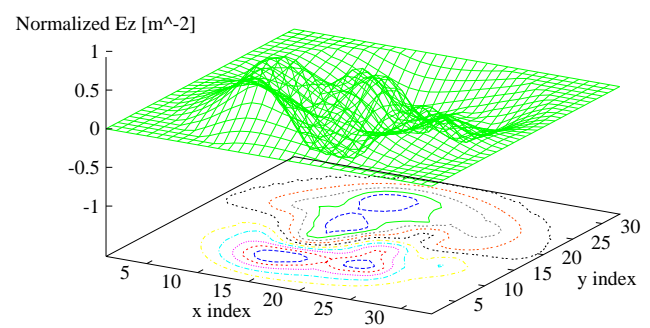

(c)

Figure 8.4: (a) $\mathrm{x}^{-}$, (b) $\mathrm{y}^{-}$, and (c) z- electric field components of the KV distribution. 


\section{Chapter 9}

\section{Beam-Cavity Interaction}

\section{$9.1 \quad$ Introduction}

This chapter summarizes the theory and formalism in the beam-cavity interaction we expect to find in relativistic-klystron two-beam accelerator systems.

\subsection{Fundamental Elements and Dynamics}

In this section we describe the main elements that take part in the dynamics, namely the cavity voltage and convection current (the beam). We will not undergo here the full discussion or derivation of the basic interaction as embodied in the circuit equation. These are well covered in the available texts [18], [19]. We will merely make their introduction, and briefly discuss some of their properties. The particular model of the current density which provides the bridge between the circuit analogy and the tracking code will be presented.

\subsubsection{Modal Elements and Dynamics}

We are specifically concerned with the interaction of the beam with the fundamental monopole mode $\left(T M_{010}\right)$ in a single standing-wave (SW) idler or output cavity. Application to transverse deflecting or focusing modes can also be handled with this formalism, with little variation.

We will assume that the fields in the cavity are dominated by a single monopole mode. A typical modal field pattern is depicted in Figure 9.1. Shown is one fourth of the geometry - the remainder of the structure can be generated by reflection through 


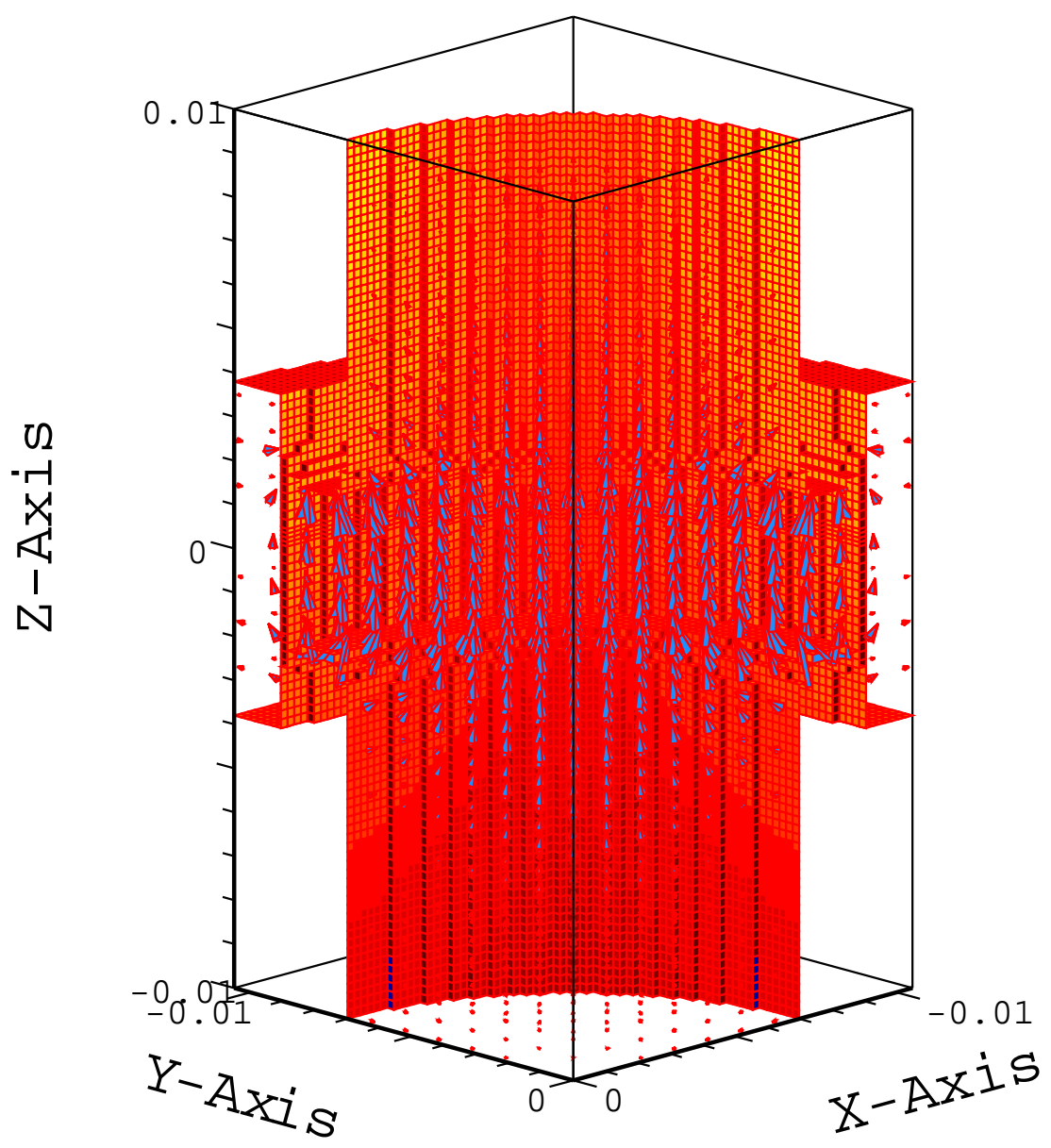

Figure 9.1: SW $T M_{010}$ mode in a symmetric idler cavity. 
both the $\mathrm{x}$ - and $\mathrm{y}$ - axes. The modal electric field is shown as a vector field against the cavity wall mesh. The beam travels parallel to the z-axis. We express the cavity electric field as a product of a time-dependent mode amplitude with a spatial mode profile (indexed by $\lambda$ ),

$$
\vec{E}(\vec{r}, t)=a_{\lambda}(t) \overrightarrow{E_{\lambda}}(\vec{r}) .
$$

The spatial profile of the mode is assumed to have the so-called 'Slater' normalization,

$$
\int_{\text {cavity }} d^{3} r \vec{E}_{\lambda}(\vec{r}) \cdot \vec{E}_{\lambda}(\vec{r})=1
$$

Other normalizations are sometimes used. Another one we employ will be described later. The spatial field profile is assumed to be static; all of the temporal behavior of the field enters through the mode amplitude. This is the quantity which is used to describe the cavity voltage as seen by the beam, and is proportional to the electric field in any output waveguide. Knowing the spatial mode profile, and the total electric field at time $\mathrm{t}$, the mode amplitude can be defined by

$$
a_{\lambda}(t)=\int_{\text {cavity }} d^{3} r \vec{E}(\vec{r}, t) \cdot \vec{E}_{\lambda}(\vec{r}) .
$$

The other dynamical quantity is the current density representing the beam travelling through the cavity structure. We define a modal current density by computing the overlap of the time-dependent current density with the spatial profile of the mode electric field,

$$
J_{\lambda}(t)=\int_{\text {cavity }} d^{3} r \vec{J}(\vec{r}, t) \cdot \vec{E}_{\lambda}(\vec{r})
$$

such that

$$
\vec{J}(\vec{r}, t)=J_{\lambda}(t) \overrightarrow{E_{\lambda}}(\vec{r})
$$

The cavity may have coupling that permits rf power to enter or leave. This coupling can be weak or strong. Viewed in terms of steady-state behavior, this coupling is typically characterized by an external-coupling quality factor, $Q_{e x t}$, of the cavity. We may also describe the coupling as an impedance transformation from the mode amplitudes (for incoming and outgoing waves) of the attached waveguide, through the coupling port surface, to the mode amplitude of the resonant cavity. This coupling surface is usually taken to lie in the transverse plane of the connecting waveguide, a short distance $(<\lambda)$ from the cavity. Slater calls this surface 'the plane of the detuned short.' In either case, we define a relationship based on the continuity of the transverse electric field at the 
port surface between the incoming $(+)$ and outgoing $(-)$ waveguide mode amplitudes, and the cavity mode amplitude,

$$
V_{1}^{+}+V_{1}^{-}=V_{1 \lambda} a_{\lambda}
$$

Here, the external coupling parameter, $V_{1 \lambda}$, measures the overlap between the cavity mode (' $\lambda$ ') and the waveguide mode (' 1 '). This assumes that the waveguide is operating with only the fundamental $\left(T E_{01}\right)$ mode present. This is a reasonable assumption for klystron input/output ports. The external coupling parameter may be related to the external Q-value of the cavity via the relation

$$
V_{1 \lambda}=\sqrt{\frac{\epsilon_{0} \omega_{\lambda} Z_{c 1}}{Q_{e x t}}}
$$

where, $Z_{c 1}$, is the characteristic impedance of the waveguide mode. This coupling can be calculated, or measured on the bench with a network analyzer.

As detailed in the references [18], [19], we write down an equivalent circuit equation describing the time evolution of the mode amplitude due to excitation by both the external rf current drive and the incoming waveguide mode, and losses from wall heating, beam loading, and coupling to the outgoing waveguide mode,

$$
\left(\frac{d^{2}}{d t^{2}}+\frac{\omega_{\lambda}}{Q_{w}} \frac{d}{d t}+\omega_{\lambda}^{2}\right) a_{\lambda}(t)=-\frac{1}{\epsilon_{0}} \frac{d}{d t} J_{\lambda}(t)+\frac{\omega_{\lambda}}{Q_{e x t}} \frac{d}{d t}\left(\frac{V_{1}^{+}-V_{1}^{-}}{V_{1 \lambda}}\right) .
$$

This equation is that of a damped harmonic oscillator, driven by two independent source terms. The oscillator term contains two external parameters related to the resonant mode: the angular frequency, $\omega_{\lambda}$; and the wall-loss quality factor, $Q_{w}$. They can be determined from cold-cavity experiments or numerical simulations.

Since the temporal structure of the fields and currents is predominantly harmonic in nature with an angular frequency, $\omega_{b}$, close to the resonant frequency of the cavity, it will be helpful to consider the corresponding frequency domain description. We may assume that the modal amplitudes and spatial profiles are real-valued functions. For quasi-steady-state harmonic oscillation at the modulation rf frequency, we express the time-dependence of the rf amplitudes as

$$
\begin{aligned}
& a_{\lambda}(t) \cong \widehat{a_{\lambda}} \cos \left(\omega_{b} t+\varphi_{\lambda}\right), \\
& J_{\lambda}(t) \cong \widehat{J_{\lambda}} \cos \left(\omega_{b} t\right),
\end{aligned}
$$




$$
V_{1}^{ \pm}(t) \cong \widehat{v}^{ \pm} \cos \left(\omega_{b} t+\varphi_{ \pm}\right)
$$

where $T_{b}=2 \pi / \omega_{b}$.

It will be convenient to also use complex-valued quantities. In this case we may express the time-dependent rf modal amplitudes as real parts (denoted by $\Re$ ) of a complex phasor,

$$
\begin{aligned}
a_{\lambda}(t) & \cong \Re\left\{\widehat{a_{\lambda}} \exp \left[-i\left(\omega_{b} t+\varphi_{\lambda}\right)\right]\right\} \\
J_{\lambda}(t) & \cong \Re\left\{\widehat{J_{\lambda}} \exp \left[-i \omega_{b} t\right]\right\}, \\
V_{1}^{ \pm}(t) & \cong \Re\left\{\widehat{v}^{ \pm} \exp \left[-i\left(\omega_{b} t+\varphi_{ \pm}\right)\right]\right\}
\end{aligned}
$$

These time-dependent mode amplitudes have constant coefficients. This is approximately correct on the time scale of the rf modulation period $\left(T_{b}\right)$, but these amplitudes also possess a slow time variation as well. This slow variation will be discussed further in Section 3.

\subsubsection{Normalization of the Cavity Electric Field}

Returning to the problem of the beam-cavity interaction, we recall that the simulation model employs a paraxial approximation which uses a description of the fields near the beamline axis. In particular, we only consider the on-axis longitudinal electric field profile and assume that it is only a function of longitudinal position $(z)$, with a separable time dependence. The values of the field and its derivatives along the axis are used to generate all other electric and magnetic field components (permitted by symmetry) near the axis by construction of the vector potential. From the point of view of the simulation, we need only those field components along the axis, a complete description of the rest of the cavity is unnecessary.

However, without the detailed description of the total electric field profile throughout the entire cavity, we are unable to normalize the modes according to the Slater prescription (9.2). But, this normalization needn't be performed in the particle tracking simulation, it may also be done when the modes are initially generated by electromagnetic codes [Superfish, URMEL, MAFIA, GdfidL, et. al.]. This can be performed through a combination of analytical modeling of the on-axis field profile with numerical calculation of the circuit [r/Q] (9.7). This relates the on-axis voltage seen by the beam to the total energy stored in the cavity.

The longitudinal component of the monopole mode electric field profile in single-cell 


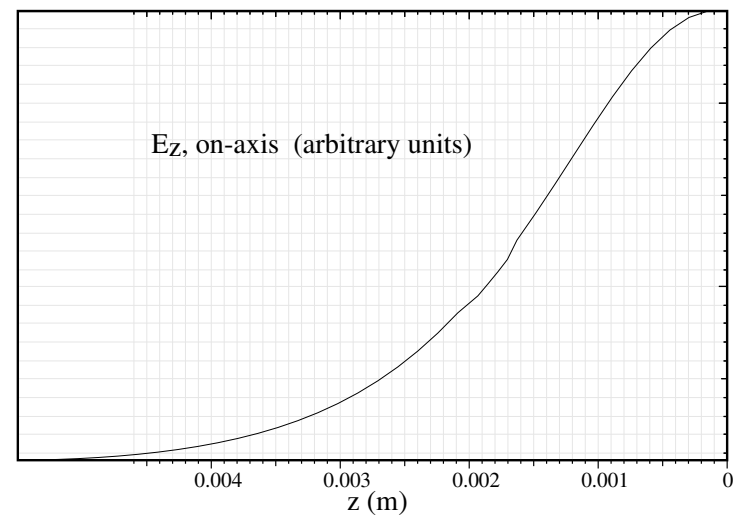

Figure 9.2: Relative strength of the longitudinal electric field component along the cavity's symmetry axis.

cavities with open beampipes is well approximated by a Gaussian distribution along the beamline axis. A typical idler cavity field profile is shown in Figure 9.2. In this picture, the cavity occupies only the first $0.5 \mathrm{~mm}$ (measured from the right border), while the beampipe occupies the rest of the length. We see that most of the mode exists in the beampipe. The standard deviation $(\sigma)$ can be obtained from simulation data by appropriate fitting procedures. The longitudinal field profile of the monopole mode can then be represented by

$$
E_{z \lambda}(z)=\frac{N_{\lambda}}{\sqrt{2 \pi} \sigma} \exp \left[-\frac{z^{2}}{2 \sigma^{2}}\right]=N_{\lambda} e_{0}(z),
$$

for a cavity centered at $z=0$. Here, $N_{\lambda}$ is the normalization constant that we wish to determine. In terms of the modal fields, the circuit [r/Q] ('r-upon-Q' or 'r-over-Q') is defined for constant velocity $\left(\beta_{z}\right)$ particles as

$$
\left[\frac{r}{Q}\right]_{\lambda}=\frac{1}{\epsilon_{0} \omega_{\lambda}}\left|\int_{\text {cavity }} d z E_{z \lambda}(z) \exp \left[i \omega_{\lambda} z / \beta_{z} c\right]\right|^{2}
$$

An accelerator $[\mathrm{R} / \mathrm{Q}]$ is also widely used in the accelerator literature. It's value is twice that of the circuit [r/Q]. We will always use 'R' for accelerator and ' $r$ ' for circuit shunt impedances and related quantities.

To derive an expression for the normalization constant, we substitute our analytical approximation for the on-axis field profile. Incurring a negligible error, we extend the limits of integration in the above integral to $\pm \infty$. We then find our normalization 
constant to be,

$$
N_{\lambda}=\left(\epsilon_{0} \omega_{\lambda}\left[\frac{r}{Q}\right]_{\lambda}\right)^{1 / 2} \exp \left[\frac{\omega_{\lambda}^{2} \sigma^{2}}{2 \beta_{z}^{2} c^{2}}\right] .
$$

This defines the connection between the modal fields used in the circuit analogy, and the fields used in determining the beam dynamics. We refer to this definition of the field, $e_{0}(z)$, as the 'line voltage' normalization such that

$$
\int_{-\infty}^{+\infty} d z e_{0}(z)=1
$$

\subsubsection{Periodic Klimontovich Current Distribution}

The connection between the discrete particle description employed by the tracking code and the current density used in the circuit equation is made by appealing to the Klimontovich distribution [88]. Since the amplitude of the modulated current density varies only very slowly on the rf time scale, the charge per bunch and the distribution function appear to be periodic when observed over a few rf periods' duration. We describe the time dependence of the charge density distribution by expanding in a Fourier series basis defined over a single rf period

$$
\begin{aligned}
& \vec{J}(\vec{r}, t) \cong \vec{z} Q_{b} \frac{1}{N} \sum_{n=1}^{N} \delta^{2}\left(\overrightarrow{r_{\perp}}-\overrightarrow{r_{\perp n}}\right) \delta\left(t-t_{n}\right), \\
& J_{z}(\vec{r}, t)=\frac{1}{2} J_{0}(\vec{r})+\sum_{l=1}^{\infty}\left[J_{l}^{+}(\vec{r}) \cos \left(l \omega_{b} t\right)+J_{l}^{-}(\vec{r}) \sin \left(l \omega_{b} t\right)\right],
\end{aligned}
$$

for $t, t_{n} \in\left[-T_{b} / 2, T_{b} / 2\right]$. Here, $\mathrm{N}$ is the number of particles carried in a simulated bunch, and $Q_{b}$ is the total charge carried by the beam current past a stationary observer in a time $T_{b}$. Hence, the beam DC current averaged over an rf period is $I_{b}=Q_{b} / T_{b}$. We assume that the particle distribution within the bunches is periodic with period $T_{b}$. Individual particles in the bunch pass the beamline position $z_{0}$ at times $t_{n}\left(z_{0}\right)$ and at transverse positions $\overrightarrow{r_{\perp n}}\left(z_{0}\right)$. The relativistic nature of the beam allows us to neglect the transverse current density components.

We compute the Fourier components of the current density,

$$
J_{l}^{ \pm}(\vec{r})=\frac{2}{T_{b}} \int_{-T_{b} / 2}^{T_{b} / 2} d t\left(\begin{array}{c}
\cos \left(l \omega_{b} t\right) \\
\sin \left(l \omega_{b} t\right)
\end{array}\right) J_{z}(\vec{r}, t) .
$$


Integrating, we obtain

$$
J_{l}^{ \pm}\left(\vec{r} ; \omega_{b}\right)=2 I_{b} \frac{1}{N} \sum_{n=1}^{N} \delta^{2}\left(\overrightarrow{r_{\perp}}-\overrightarrow{r_{\perp n}}\right)\left(\begin{array}{c}
\cos \left(l \omega_{b} t_{n}\right) \\
\sin \left(l \omega_{b} t_{n}\right)
\end{array}\right)
$$

Re-assembling the Fourier components,

$$
\begin{aligned}
J_{z}(\vec{r}, t) & =I_{b} \frac{1}{N} \sum_{n=1}^{N} \delta^{2}\left(\overrightarrow{r_{\perp}}-\overrightarrow{r_{\perp n}}\right)\left\{1+2 \sum_{l=1}^{\infty}\left[\cos \left(l \omega_{b} t\right) \cos \left(l \omega_{b} t_{n}\right)+\sin \left(l \omega_{b} t\right) \sin \left(l \omega_{b} t_{n}\right)\right]\right\} \\
& =I_{b} \frac{1}{N} \sum_{n=1}^{N} \delta^{2}\left(\overrightarrow{r_{\perp}}-\overrightarrow{r_{\perp n}}\right)\left\{1+2 \sum_{l=1}^{\infty} \cos \left[l \omega_{b}\left(t-t_{n}\right)\right]\right\} .
\end{aligned}
$$

The modal current density at time $t_{0}$ is now calculated to be

$$
\begin{aligned}
J_{\lambda}\left(t_{0}\right)= & \int_{\text {cavity }} d^{3} r J_{z}\left(\vec{r}, t_{0}\right) E_{z \lambda}(\vec{r}) \\
J_{\lambda}\left(t_{0}\right)= & I_{b} \frac{1}{N} \sum_{n=1}^{N} \int_{\text {cavity }} d z E_{z \lambda}\left(\overrightarrow{r_{\perp n}}, z\right)\left\{1+2 \sum_{l=1}^{\infty} \cos \left[l \omega_{b}\left(t_{0}-t_{n}\right)\right]\right\} \\
J_{\lambda}\left(t_{0}\right)= & I_{b}\left\langle\int_{\text {cavity }} d z E_{z \lambda}\left(\overrightarrow{r_{\perp}}, z\right)\right\rangle+ \\
& 2 I_{b} \sum_{l=1}^{\infty}\left\langle\int_{\text {cavity }} d z E_{z \lambda}\left(\overrightarrow{r_{\perp}}, z\right) \cos \left[l \omega_{b}\left(t_{0}-t(z)\right)\right]\right\rangle .
\end{aligned}
$$

We have denoted with angular brackets, $\langle>$, an average over the bunch distribution. Here, $t(z)$ is the arrival time at beamline position $z$ with transverse offset $\overrightarrow{r_{\perp}}$ of an element of the current density. Again, it will be convenient to use the real parts of complex-valued quantities,

$J_{\lambda}(t)=I_{b}\left\langle\int_{\text {cavity }} d z E_{z \lambda}\left(\overrightarrow{r_{\perp}}, z\right)\right\rangle+2 I_{b} \sum_{l=1}^{\infty} \Re\left\{\exp \left[-i l \omega_{b} t\right]\left\langle\int_{\text {cavity }} d z E_{z \lambda}\left(\overrightarrow{r_{\perp}}, z\right) \exp \left[i l \omega_{b} t(z)\right]\right\rangle\right\}$.

This particular integral of the modal electric field occurs so often that we will just define,

$$
\widetilde{w}\left(\omega, \overrightarrow{r_{\perp}}\right)=\int_{\text {cavity }} d z E_{z \lambda}\left(\overrightarrow{r_{\perp n}}, z\right) \exp [-i \omega t(z)] .
$$

The phase convention employed in this definition corresponds to $t\left(z_{\text {mid }}\right)=0$, where $z_{\text {mid }}$ is the longitudinal position at the center of the cavity. This convention is applied to the fiducial orbit only, all other particle orbits introduce relative phase changes.

The particular function defined by (9.9) plays an extremely important role in the 
dynamics of the beam and the evolution of the cavity field amplitude. It serves to define the cavity voltage, and hence the mode $[\mathrm{R} / \mathrm{Q}]$ and shunt impedance. It contains transit time effects, and when applied to beam particle trajectories in the presence of a background rf field, it will then compute beam loading contributions to the shunt impedance and the net energy deposited into the mode. We will refer to it as the mode transit function.

The modal current density can then be expressed as

$$
J_{\lambda}(t)=I_{b}\left\langle\tilde{w}^{\dagger}\left(\omega=0, \overrightarrow{r_{\perp}}\right)\right\rangle+2 I_{b} \sum_{l=1}^{\infty} \Re\left\{\exp \left[-i l \omega_{b} t\right]\left\langle\tilde{w}^{\dagger}\left(l \omega_{b}, \overrightarrow{r_{\perp}}\right)\right\rangle\right\}
$$

Comparing this last form to (9.5), we make the identification of the rf modal current density amplitude,

$$
\widehat{J_{\lambda}}=2 I_{b} \sum_{l=1}^{\infty}\left\langle\widetilde{w}^{\dagger}\left(l \omega_{b}, \overrightarrow{r_{\perp}}\right) \exp \left[-i l \omega_{b} t\right]\right\rangle .
$$

For the beams we consider here, only the $l=1$ term is necessary to retain for the monopole mode. Higher-order azimuthal modes may couple to higher harmonic components in the beam's spectrum.

\subsection{Analysis of the Circuit Equation}

In this section we will employ the elements developed in Section 9.2. We will assume that there exist two distinct time-scales of interest. A fast time-scale, where variations are seen to occur within an rf period, and a slow time-scale. The latter can be taken to be the fill time of the cavity $\left(T_{F}=T_{b} Q_{\lambda} / \pi\right)$, the rise time of the driving current, or some other relevant time-scale. The mode amplitude and current density are modulated at the fast time-scale. But the evolution of the amplitude as well as any phase drift occurs on the slow time-scale. As a result, we may re-write the governing circuit equation in terms of these slowly-varying quantities and the slow time-scale.

\subsubsection{Slow Time-Scale Equation of Motion}

From here on, every operation we perform will be linear. We will work, then, with the complex-valued quantities $(9.5,9.10)$ defined in Section 9.2, provided that we only consider the real parts of any quantity at the end of the calculation. We introduce slow 
time variations into the modal amplitudes and phases,

$$
\begin{aligned}
\widehat{a_{\lambda}}=\widehat{a_{\lambda}}(t) \quad, \quad \varphi_{\lambda}=\varphi_{\lambda}(t), \\
\widehat{J_{\lambda}}=2 I_{b}(t)\left\langle\widetilde{w}^{\dagger}\right\rangle, \\
\widehat{v^{ \pm}}=\widehat{v^{ \pm}}(t) \quad, \quad \varphi_{ \pm}=\varphi_{ \pm}(t),
\end{aligned}
$$

where the time-dependent quantities are all real-valued functions. These functions are required to be slowly varying in time with respect to the rf period. For a quantity $f(t)$, this means that $\left|\frac{d f}{d t}\right| \ll\left|f \omega_{b}\right|$. We substitute these into the circuit equation (9.4), and neglect second-order time derivatives of slowly-varying quantities,

$$
\begin{aligned}
\left(\omega_{\lambda}^{2}-\omega^{2}-\frac{i \omega \omega_{\lambda}}{Q_{w}}\right)\left(\widehat{a_{\lambda}} e^{-i \varphi_{\lambda}}\right)+ & \left(\frac{\omega_{\lambda}}{Q_{w}}-2 i \omega\right) \frac{d}{d t}\left(\widehat{a_{\lambda}} e^{-i \varphi_{\lambda}}\right)= \\
= & \frac{\omega_{\lambda}}{Q_{e x t} V_{1 \lambda}}\left(\frac{d}{d t}-i \omega\right)\left(\widehat{v}^{+} e^{-i \varphi_{+}}-\widehat{v}^{-} e^{-i \varphi_{-}}\right) \\
& -2 \frac{\left\langle\widetilde{w}^{\dagger}\right\rangle}{\epsilon_{0}}\left(\frac{d}{d t} I_{b}-i \omega I_{b}\right) .
\end{aligned}
$$

This equation can be simplified by introducing a voltage normalization. We define an on-axis cavity circuit voltage, and forward and reverse port voltages in the mode transit normalization via

$$
\begin{aligned}
\widetilde{V_{c}} & =\left(\widetilde{w_{0}} \widehat{a_{\lambda}} e^{-i \varphi_{\lambda}}\right), \\
\widetilde{V_{F}} & =\left(\frac{\widetilde{w_{0}} \widehat{v}^{+} e^{-i \varphi_{+}}}{V_{1 \lambda}}\right), \\
\widetilde{V_{R}} & =\left(\frac{\widetilde{w_{0}} \widehat{v}^{-} e^{-i \varphi_{-}}}{V_{1 \lambda}}\right),
\end{aligned}
$$

where $\widetilde{w_{0}}=\widetilde{w}\left(\overrightarrow{r_{\perp}}=0, \omega_{b}\right)$. In this normalization, the continuity of the transverse electric field at the port plane is expressed as

$$
\widetilde{V_{c}}=\widetilde{V_{F}}+\widetilde{V_{R}}
$$

In the absence of any beam the mode transit function, $\widetilde{w}$, is undefined. However, there may still be fields present in the cavity as well as coupling through the ports. In 
this case, we will utilize a different scheme, the port overlap normalization,

$$
\begin{aligned}
\widetilde{V_{c}} & =\left(V_{1 \lambda} \widehat{a_{\lambda}} e^{-i \varphi_{\lambda}}\right), \\
\widetilde{V_{F}} & =\left(\widehat{v}^{+} e^{-i \varphi_{+}}\right), \\
\widetilde{V_{R}} & =\left(\hat{v}^{-} e^{-i \varphi_{-}}\right) .
\end{aligned}
$$

As defined, these voltages are complex-valued. The bunch-averaged accelerator shunt impedance is defined through $R_{b}=Q_{\lambda}\langle[R / Q]\rangle$, where

$$
\left\langle\left[\frac{R}{Q}\right]\right\rangle=\frac{2 \widetilde{w_{0}}\left\langle\widetilde{w}^{\dagger}\right\rangle}{\epsilon_{0} \omega_{\lambda}} .
$$

With these definitions, the circuit equation (9.11) becomes (in the mode transit normalization)

$$
\begin{gathered}
\left(\omega_{\lambda}^{2}-\omega^{2}-\frac{i \omega \omega_{\lambda}}{Q_{w}}\right) \widetilde{V_{c}}+\left(\frac{\omega_{\lambda}}{Q_{w}}-2 i \omega\right) \frac{d}{d t} \widetilde{V_{c}}= \\
=\frac{\omega_{\lambda}}{Q_{e x t}}\left(\frac{d}{d t}-i \omega\right)\left(\widetilde{V_{F}}-\widetilde{V_{R}}\right) \\
-\omega_{\lambda}\left\langle\left[\frac{R}{Q}\right]\right\rangle\left(\frac{d}{d t} I_{b}-i \omega I_{b}\right) .
\end{gathered}
$$

Using the continuity condition (9.13), we may express the reverse voltage in terms of the cavity and forward voltages. Doing so, the circuit equation becomes

$$
\begin{aligned}
\left(\omega_{\lambda}^{2}-\omega^{2}-\frac{i \omega \omega_{\lambda}}{Q_{L}}\right) \widetilde{V_{c}} & +\left(\frac{\omega_{\lambda}}{Q_{L}}-2 i \omega\right) \frac{d}{d t} \widetilde{V_{c}}= \\
& =2 \frac{\omega_{\lambda}}{Q_{e x t}}\left(\frac{d}{d t}-i \omega\right) \widetilde{V_{F}} \\
-\omega_{\lambda} & \left\langle\left[\frac{R}{Q}\right]\right\rangle\left(\frac{d}{d t} I_{b}-i \omega I_{b}\right) .
\end{aligned}
$$

The loaded quality factor, $Q_{L}$, has been introduced with the definition

$$
\frac{1}{Q_{L}}=\frac{1}{Q_{w}}+\frac{1}{Q_{e x t}}
$$

which is a statement of net power loss in the cavity's fields in the absence of beam coupling.

From standard microwave terminology, we recall the definitions of the tuning angle, 
$\psi$, cavity fill-time, $T_{F}$, and coupling parameter, $\beta$,

$$
\begin{aligned}
\tan \psi & =Q_{L}\left(\frac{\omega_{\lambda}}{\omega}-\frac{\omega}{\omega_{\lambda}}\right), \\
T_{F} & =\frac{2 Q_{L}}{\omega_{\lambda}}, \\
\beta & =\frac{Q_{w}}{Q_{e x t}},
\end{aligned}
$$

and introduce the phase change in a fill time, $\nu=\omega T_{F}$. The circuit equation then becomes,

$$
\begin{aligned}
-i \frac{\omega \omega_{\lambda}}{Q_{L}}(1+i \tan \psi) \widetilde{V_{c}} & +\left(\frac{\omega_{\lambda}}{Q_{L}}-2 i \omega\right) \frac{d}{d t} \widetilde{V_{c}}= \\
& =2 \frac{\omega_{\lambda}}{Q_{e x t}}\left(\frac{d}{d t}-i \omega\right) \widetilde{V_{F}} \\
-\omega_{\lambda} & \left\langle\left[\frac{R}{Q}\right]\right\rangle\left(\frac{d}{d t}-i \omega\right) I_{b} .
\end{aligned}
$$

We will neglect terms of order $O\left(\frac{1}{4 Q_{w}^{2}}\right)$, and express derivatives in terms of $\tau=t / T_{F}$ (using primes to indicate derivation). We also introduce the supplementary parameters $\mu=\frac{1-i / \nu}{1+\nu^{2}}$ and $\alpha=\mu(1+i \tan \psi)$.

In Section 4, it will be shown that the shunt impedance as defined here is a complexvalued quantity, but that it can be expressed as,

$$
\left\langle\left[\frac{R}{Q}\right]\right\rangle=\left[\frac{R}{Q}\right]_{\lambda} F_{b},
$$

where

$$
\left[\frac{R}{Q}\right]_{\lambda}=2 \frac{\left|\widetilde{w_{0}}\right|^{2}}{\epsilon_{0} \omega_{\lambda}}
$$

is a manifestly real-valued quantity, and $F_{b}$ is a complex-valued bunch distributiondependent form-factor, with magnitude of order unity. This form factor provides a measure of the spacetime overlap of the bunch distribution with the mode on the time-scale of the rf period. It's calculation takes into account the individual electron trajectories, and hence accounts for beam-loading effects. With the bunch-distribution independent shunt impedance,

$$
R_{\lambda}=Q_{L}\left[\frac{R}{Q}\right]_{\lambda}
$$


the circuit equation takes its final form,

$$
\begin{aligned}
{\widetilde{V_{c}}}^{\prime}+\alpha \widetilde{V_{c}} & =\frac{2 \beta}{\beta+1} \mu\left(\widetilde{V_{F}}+\frac{i}{\nu}{\widetilde{V_{F}}}^{\prime}\right)-R_{\lambda} F_{b} \mu\left(I_{b}+\frac{i}{\nu} I_{b}^{\prime}\right), \\
\widetilde{V_{R}} & =\widetilde{V_{c}}-\widetilde{V_{F}} .
\end{aligned}
$$

This is the main result of this section. We can solve (9.17) for the special case of linear variation of the current with time. This will give us solutions valid for both the initial and final ramping portions of the beam current as well as the flat-top. We do not attempt to find a global solution over time, which requires inclusion of the self-consistent interaction of the cavity back upon the beam. Rather, we will seek a local solution, valid only over a short time duration (though still long compared to the fast time scale), as an approximation to use within the numerical simulation.

\subsubsection{Analytic Solution for Short (Slow Scale) Time Duration}

The circuit equation above (9.17) can be simplified slightly by introducing parameters that absorb the local time-dependence of the beam current and forward power,

$$
\begin{aligned}
B_{b}(\tau) & =1+\frac{i}{\nu} \frac{I_{b}^{\prime}(\tau)}{I_{b}(\tau)} \\
B_{F}(\tau) & =1+\frac{i}{\nu} \frac{\widetilde{V_{F}}(\tau)}{\widetilde{V_{F}}(\tau)}
\end{aligned}
$$

Both these factors become unity under steady-state conditions. Equation (9.17) is now

$$
{\widetilde{V_{c}}}^{\prime}+\alpha \widetilde{V_{c}}=\frac{2 \beta}{\beta+1} \mu B_{F} \widetilde{V_{F}}-R_{\lambda} F_{b} \mu B_{b} I_{b}
$$

We integrate this differential equation over a small time interval. We assume that the bunch dependent form factor $\left(F_{b}\right)$ is nearly constant, which is a good approximation provided the cavity voltage does not change by a significant fraction of the beam voltage over the interval of integration. In other words, we assume the intra-bunch particle motion remains nearly identical from bunch to bunch, so that the bunch-averaged quantities like emittance and energy spread do not appreciably vary over the interval of integration. This particle motion is calculated by the tracking algorithm based upon fields excited from the passage of previous bunches and the coupling of external rf power into the cavity. We allow for a linear time dependence of the current and forward power, so that both $B_{b}$ and $B_{F}$ are constant over the time interval. After integrating the equa- 
tion between times $\tau_{1}$ and $\tau_{2}$, and dropping terms of order $O\left(\frac{1}{4 Q_{w}^{2}}\right)$, we find our short term solution

$$
\begin{aligned}
\widetilde{V_{c}}\left(\tau_{2}\right)= & \widetilde{V_{c}}\left(\tau_{1}\right) e^{-\alpha\left(\tau_{2}-\tau_{1}\right)} \\
& +\left[B_{F} \frac{2 \beta}{\beta+1} \widetilde{V_{F}}\left(\tau_{1}\right)-R_{\lambda} F_{b} B_{b} I_{b}\left(\tau_{1}\right)\right] \cos \psi e^{-i \psi}\left[1-e^{-\alpha\left(\tau_{2}-\tau_{1}\right)}\right] .
\end{aligned}
$$

We define a (complex-valued) beam impedance by $Z_{b}=R_{\lambda} F_{b} B_{b}$. The solution to the circuit voltage is then

$$
\begin{aligned}
\widetilde{V_{c}}\left(\tau_{2}\right) & =\widetilde{V_{c}}\left(\tau_{1}\right) e^{-\alpha\left(\tau_{2}-\tau_{1}\right)} \\
& +\left[B_{F} \frac{2 \beta}{\beta+1} \widetilde{V_{F}}\left(\tau_{1}\right)-Z_{b} I_{b}\left(\tau_{1}\right)\right] \cos \psi e^{-i \psi}\left[1-e^{-\alpha\left(\tau_{2}-\tau_{1}\right)}\right]
\end{aligned}
$$

This solution is seen to track both losses and phase shifts due to beam loading and phase slippage on the long time-scale.

\subsubsection{Observed Fields and Power Flow}

The physical observables, the time-dependent rf mode amplitudes, are derived from these circuit voltages. The observables have the time dependence

$$
\begin{aligned}
a_{\lambda}(t) & \cong \widehat{a_{\lambda}}(t) \cos \left(\omega_{b} t+\varphi_{\lambda}(t)\right), \\
J_{\lambda}(t) & \cong \widehat{J_{\lambda}}(t) \cos \left(\omega_{b} t\right), \\
V_{1}^{ \pm}(t) & \cong \widehat{v}^{ \pm}(t) \cos \left(\omega_{b} t+\varphi_{ \pm}(t)\right) .
\end{aligned}
$$

Depending upon the voltage normalization scheme $(9.12,9.14)$, the amplitudes and phases of the fields and modes are then

$$
\begin{array}{r}
\widehat{a}(t)=\left|\frac{\widetilde{V_{c}}}{\widetilde{w_{0}}}\right| \quad, \quad \varphi_{\lambda}(t)=-L\left(\frac{\widetilde{V_{c}}}{\widetilde{w_{0}}}\right) \\
\widehat{v_{ \pm}}(t)=V_{1 \lambda}\left|\frac{\widetilde{V_{F, R}}}{\widetilde{w_{0}}}\right| \quad, \quad \varphi_{ \pm}(t)=-L\left(\frac{\widetilde{V_{F, R}}}{\widetilde{w_{0}}}\right)
\end{array}
$$

in the mode transit normalization, and

$$
\begin{gathered}
\widehat{a}(t)=\left|\frac{\widetilde{V_{c}}}{V_{1 \lambda}}\right| \quad, \quad \varphi_{\lambda}(t)=-\angle\left(\widetilde{V_{c}}\right) \\
\widehat{v_{ \pm}}(t)=\left|\widehat{V_{F, R}}\right| \quad, \quad \varphi_{ \pm}(t)=-\angle\left(\widetilde{V_{F, R}}\right)
\end{gathered}
$$


in the port overlap normalization.

Of interest is the amount of rf power flowing into and out of the cavity. This power flow is derived from the Poynting flux. The waveguide modes are assumed to be normalized such that the transverse electric fields satisfy a relation similar to (9.2). The net rms power flowing in the waveguide, again assuming a single mode, can then be shown to be

$$
\bar{P}_{\text {guide }}=\frac{\left(\widehat{v^{+}}\right)^{2}-\left(\widehat{v^{-}}\right)^{2}}{2 Z_{c 1}}
$$

with

$$
\begin{aligned}
& \bar{P}_{+}=\frac{\left(\widehat{v^{+}}\right)^{2}}{2 Z_{c 1}}, \\
& \bar{P}_{-}=\frac{\left(\widehat{v^{-}}\right)^{2}}{2 Z_{c 1}},
\end{aligned}
$$

denoting the forward and reverse rms power flows in the connecting waveguide, respectively. Translating this into the normalization schemes gives

$$
\begin{gathered}
\bar{P}_{+}=\frac{\epsilon_{0} \omega_{\lambda}}{2 Q_{e x t}}\left|\frac{\widetilde{V_{F}}}{\widetilde{w_{0}}}\right|^{2} \quad, \quad \bar{P}_{-}=\frac{\epsilon_{0} \omega_{\lambda}}{2 Q_{e x t}}\left|\frac{\widetilde{V_{c}}-\widetilde{V_{F}}}{\widetilde{w_{0}}}\right|^{2} ; \\
\bar{P}_{+}=\frac{\left|\widetilde{V_{F}}\right|^{2}}{2 Z_{c 1}} \quad, \quad \bar{P}_{-}=\frac{\left|\widetilde{V_{c}}-\widetilde{V_{F}}\right|^{2}}{2 Z_{c 1}},
\end{gathered}
$$

in the mode transit and port overlap schemes, respectively. Note that the reverse voltage has been expressed in terms of the forward and cavity voltages.

The rms value of the energy stored in the cavity is given by

$$
U_{c}=\frac{\epsilon_{0}}{2}\left|\widehat{a_{\lambda}}\right|^{2} .
$$

The rms power shunted to the walls and to the reverse waveguide mode can derived by appealing to the definition of the quality-factor,

$$
\bar{P}=\frac{\omega_{\lambda} U_{c}}{Q}
$$

Hence, the wall-loss power and the reverse power are given by

$$
\bar{P}_{w}=\frac{\omega_{\lambda} U_{c}}{Q_{w}}=\frac{\epsilon_{0} \omega_{\lambda}}{2 Q_{w}}\left|\widehat{a_{\lambda}}\right|^{2}
$$




$$
\bar{P}_{-}=\frac{\omega_{\lambda} U_{c}}{Q_{e x t}}=\frac{\epsilon_{0} \omega_{\lambda}}{2 Q_{e x t}}\left|\widehat{a_{\lambda}}\right|^{2}
$$

as expected. From energy conservation, the sum of these shunted powers must equal the power obtained from the beam and the forward power,

$$
\bar{P}_{b}+\bar{P}_{+}=\bar{P}_{w}+\bar{P}_{-} .
$$

\subsubsection{Steady-State Behavior}

In the limit of steady-state behavior, the differential circuit equation (9.17) becomes the algebraic equation

$$
\begin{aligned}
\widetilde{V_{c}} & =\left(\frac{2 \beta}{\beta+1} \widetilde{V_{F}}-Z_{b} I_{b}\right) \cos \psi e^{-i \psi}, \\
\widetilde{V_{R}} & =\widetilde{V_{c}}-\widetilde{V_{F}} .
\end{aligned}
$$

We compute the reverse power flow in terms of the forward power flow and beam coupling,

$$
\begin{aligned}
\bar{P}_{-}= & \frac{\epsilon_{0} \omega}{2 Q_{\text {ext }}}\left|\left(\frac{2 \beta}{\beta+1} \frac{\widetilde{V_{F}}}{\widetilde{w_{0}}}-R_{\lambda} I_{b} \frac{F_{b}}{\widetilde{w_{0}}}\right) \cos \psi e^{-i \psi}-\frac{\widetilde{V_{F}}}{\widetilde{w_{0}}}\right|^{2} \\
= & \frac{\epsilon_{0} \omega}{2 Q_{\text {ext }}}\left\{\cos ^{2} \psi\left|\left(\frac{2 \beta}{\beta+1} \frac{\widetilde{V_{F}}}{\widetilde{w_{0}}}-R_{\lambda} I_{b} \frac{F_{b}}{\widetilde{w_{0}}}\right)\right|^{2}+\left|\frac{\widetilde{V_{F}}}{\widetilde{w_{0}}}\right|^{2}\right\} \\
& +\frac{\epsilon_{0} \omega}{2 Q_{\text {ext }}}\left\{-2 \Re\left[\left(\frac{2 \beta}{\beta+1} \frac{\widetilde{V_{F}}}{\widetilde{w_{0}}}-R_{\lambda} I_{b} \frac{F_{b}}{\widetilde{w_{0}}}\right) \cos \psi e^{-i \psi} \frac{{\widetilde{V_{F}}}^{\dagger}}{\widetilde{w}_{0}^{\dagger}}\right]\right\} .
\end{aligned}
$$

The complex-valued phasors are represented by (9.18) or (9.19), and

$$
\frac{F_{b}}{\widetilde{w_{0}}}=\left|\frac{F_{b}}{\widetilde{w_{0}}}\right| e^{-i \varphi_{b}}
$$

The revese power flow is then

$$
\begin{aligned}
\bar{P}_{-}= & \bar{P}_{+}\left[\sin ^{2} \psi+\left(\frac{1-\beta}{1+\beta}\right) \cos ^{2} \psi\right]+\frac{Q_{L}}{Q_{e x t}}\left|F_{b}\right|^{2} I_{b}^{2} R_{\lambda} \cos ^{2} \psi \\
& +\sqrt{\bar{P}_{+} \frac{Q_{L}}{Q_{e x t}}\left|F_{b}\right|^{2} I_{b}^{2} R_{\lambda} \cos ^{2} \psi}\left[\frac{2 \beta}{\beta+1} \cos \psi \cos \left(\varphi_{b}-\varphi_{+}\right)-\cos \left(\psi+\varphi_{b}-\varphi_{+}\right)\right] .
\end{aligned}
$$




\subsection{Internal Bunch Dynamics and the Averaged Shunt Impedance}

In this section we calculate the bunch-averaged value of the shunt impedance. This will necessitate a somewhat closer inspection of the intra-bunch particle dynamics as the beam crosses the cavity. In particular, we need to examine slippage effects that determine the beam loading effects in the interaction.

From our definition of the shunt impedance (9.15) we recall

$$
\left\langle\left[\frac{R}{Q}\right]\right\rangle=2 \frac{\widetilde{w}_{0}\left\langle\widetilde{w}^{\dagger}\right\rangle}{\epsilon_{0} \omega_{\lambda}},
$$

where

$$
\widetilde{w}\left(\omega_{b}, \vec{r}_{\perp}\right)=\int_{\text {cavity }} d z E_{z \lambda}\left(\vec{r}_{\perp}, z\right) \exp \left[-i \omega_{b} t(z)\right]
$$

and $\widetilde{w}_{0}=\widetilde{w}\left(\omega_{b}, \vec{r}_{\perp}=0\right)$. In the usual linac formulation, the function $\widetilde{w}_{0}$ is calculated by assuming a constant velocity, $\beta_{0}$, of particles through the cavity. In that case $t(z)=$ $t_{0}+\left(z-z_{0}\right) /\left(\beta_{0} c\right)$. Here, we allow for intra-bunch particle motion resulting from a finite beam energy spread, and from the influence of rf fields pre-existing with the cavity. We determine the value of $t$ from the fiducial orbit.

To calculate the trajectory dependent mode transit function, $\widetilde{w}^{\dagger}\left(\omega_{b}, \vec{r}_{\perp}\right)$, we maintain the spirit of our tracking code and appeal to the power series expansion. For a SW monopole mode, we use

$$
E_{z \lambda}\left(\overrightarrow{r_{\perp}}, z\right)=f_{0}(z)+r_{\perp}^{2} f_{2}(z)+r_{\perp}^{4} f_{4}(z)+\cdots
$$

where $r_{\perp}^{2}=x^{2}+y^{2}$, and the auxiliary functions are (primes denoting total derivatives with respect to $\mathrm{z}$ )

$$
\begin{aligned}
& f_{0}(z)=E_{z \lambda}\left(\overrightarrow{r_{\perp}}=0, z\right)=N_{\lambda} e_{0}(z), \\
& f_{2}(z)=-1 / 4 N_{\lambda}\left(e_{0}^{\prime \prime}+k_{0}^{2} e_{0}\right), \\
& f_{4}(z)=1 / 64 N_{\lambda}\left(e_{0}^{(i v)}+2 k_{0}^{2} e_{0}^{\prime \prime}+k_{0}^{4} e_{0}\right),
\end{aligned}
$$

and $k_{0}=\omega_{\lambda} / c$. This particular set of series coefficients is a consequence of the requirement that $E_{z}\left(\overrightarrow{r_{\perp}}, z\right)$ satisfy the wave equation. As shown, the mode field profile $E_{z \lambda}\left(\overrightarrow{r_{\perp}}, z\right)$ carries the 'Slater' normalization, while the field $e_{0}(z)$ has the 'line voltage' normalization. With the form of the mode profile expressed in (9.20), we re-write the 
mode transit functions as

$$
\begin{aligned}
& \widetilde{w}_{0}=\int_{\text {cavity }} d z f_{0}(z) \exp \left[-i \omega_{b} t(z)\right] \\
& \widetilde{w}^{\dagger} \cong \int_{\text {cavity }} d z\left(f_{0}(z)+r_{\perp}^{2} f_{2}(z)+r_{\perp}^{4} f_{4}(z)\right) \exp \left[i \omega_{b} t(z)\right]
\end{aligned}
$$

In evaluating these integrals during the simulation, we break up the interval covering the entire longitudinal length of the cavity into a set of shorter sub-intervals (on the order of 10). In each sub-interval, we will assume that individual particle transverse coordinates do not change appreciably. Then, for each particle in the simulation, we replace $r_{\perp}^{2}$ and $r_{\perp}^{4}$ by the values $\overline{r_{\perp}^{2}}$ and $\overline{r_{\perp}^{4}}$, averaged over the sub-interval. Equation (9.21) can be re-written as

$$
\begin{aligned}
\widetilde{w}^{\dagger} \cong \int_{\text {cavity }} d z f_{0}(z) \exp \left[i \omega_{b} t(z)\right] \\
+\overline{r_{\perp}^{2}} \int_{\text {cavity }} d z f_{2}(z) \exp \left[i \omega_{b} t(z)\right] \\
+\overline{r_{\perp}^{4}} \int_{\text {cavity }} d z f_{4}(z) \exp \left[i \omega_{b} t(z)\right]
\end{aligned}
$$

In these integrals, the arrival time in the exponential is a dynamical coordinate of each particle. We may express the arrival time of an individual particle as the sum of the fiducial arrival time with the time deviation coordinate of the particle, $t=t_{0}+\widehat{t}$.

In computing the bunch averages over the sub-intervals, we also assume that the individual particle longitudinal phases (ie. arrival times) remain nearly constant with respect to the fiducial. We will use the notation

$$
\begin{aligned}
\omega t(z) & =(\omega / c) c t(z) \\
& =k s(z) \\
& =k s_{0}(z)+k \widehat{s}(z) \\
& \approx k s_{0}(z)+k \widehat{s},
\end{aligned}
$$

where $s_{0}(z)$ is the scaled arrival time coordinate for the fiducial at beamline position $\mathrm{z}$, and $\widehat{s}$ measures the deviation in scaled arrival time between individual particles and the fiducial. From (9.22), the 'kth'-order mode transit function is

$$
\int_{\text {cavity }} d z f_{k}(z) \exp \left[i \omega_{b} t(z)\right] \cong \exp \left[i k_{b} \widehat{s}\right] \int_{\text {cavity }} d z f_{k}(z) \exp \left[i k_{b} s_{0}(z)\right]
$$




$$
=\exp \left[i k_{b} \widehat{s}\right] \widetilde{w}_{k}^{\dagger}
$$

where $(\mathrm{k}=0,2,4)$, and the integrals are to be performed over the fiducial quantities only.

For the beams of interest to us for RK-TBA devices, the longitudinal phase space is characterized by bunches which subtend a significant fraction of the rf wavelength, with small instantaneous energy spread and significant correlation between energy and arrival time. By retaining nonlinearities in the equations of motion to only low orders, large errors are accumulated for particle orbits lying far from the fiducial. We ameliorate this situation by computing the transfer maps induced by multiple fiducials. The original fiducial (called the 'central' fiducial) is retained to provide continuity, but temporary fiducials are added to more accurately describe the entire bunch dynamics by sampling different portions of the longitudinal phase space. This can be rapidly evaluated in the simulation environment.

The bunch-averaged shunt impedance is evaluated by taking averages over the spatial and phase coordinates of the particles in the bunch

$$
\begin{aligned}
\left\langle R_{\lambda}\right\rangle & =Q_{\lambda}\left\langle\left[\frac{R}{Q}\right]\right\rangle=Q_{\lambda}\left[\frac{R}{Q}\right]_{\lambda} F_{b} \\
& =Q_{\lambda}\left\{\frac{2\left|\widetilde{w}_{0}\right|^{2}}{\epsilon_{0} \omega_{\lambda}}\right\}\left\{\left\langle\exp \left[i k_{b} \widehat{s}\right]\right\rangle+\frac{\widetilde{w}_{2}^{\dagger}}{\widetilde{w}_{0}^{\dagger}}\left\langle\overline{r_{\perp}^{2}} \exp \left[i k_{b} \widehat{s}\right]\right\rangle+\frac{\widetilde{w}_{4}^{\dagger}}{\widetilde{w}_{0}^{\dagger}}\left\langle\overline{r_{\perp}^{4}} \exp \left[i k_{b} \widehat{s}\right]\right\rangle\right\} .
\end{aligned}
$$

This form serves to define the bunch-averaged form factor, $F_{b}$. 


\section{Chapter 10}

\section{Multi-Fiducial Extensions}

\subsection{Introduction}

The multi-fiducial technique is a generalization of the single-particle map to a multiparticle map. One of the main disadvantages of tracking by mapping can be seen from the following considerations. The resultant map is fundamentally a power series expansion about the initial and final coordinates of the fiducial orbit. When the extent of the beam distribution in phase space is no longer 'small' in some sense, then the map generated about the given fiducial orbit loses accuracy when applied to the outlying particles. There are various solutions that may be applied to this problem. The pre-calculated fiducial may not faithfully represent the orbit of the beam centroid, but another fiducial may be found which does. The order of non-linearity carried by the calculation may be too low to adequately describe the given external field structure. Increasing the order of the calculation may be sufficient in this case. However, these 'fixes' make for a good solution only when the beam occupies a small enough region of phase space such that a single fiducial orbit and the map it induces captures the essential dynamics.

In many applications of intense, modulated beams, however, this is not the case. These beams are most often present in single pass, linear beamlines, with constantly changing parameters ${ }^{1}$. The beams may also have a relatively long pulse length with respect to any time-dependent rf fields they encounter. To sufficiently capture that interaction via a single map would require a degree of non-linearity far too high, and

\footnotetext{
${ }^{1}$ Hence, there is not usually a '1-period' or 'N-period' map, and Fourier techniques in map analysis are hereby de-emphasized. In fact, these maps can be time-dependent, as well as z-dependent, and a steady-state of the external fields is unlikely to exist. The individual map, then, has lost any unique position in analyzing the 'global' dynamics of the system. This point has been made apparent already by Forest [89].
} 
involve the computation of too many map coefficients, that the intrinsic efficiency of the method would be quickly lost. This problem is only compounded when self-field effects are included.

\subsection{Construction of a Multi-Fiducial Map}

The construction and evaluation of the multi-fiducial map upon the particle coordinates is straightforward. Algorithmically, it may be described by a sequence of simple steps. In any calculation, the beamline under consideration is initially divided into a set of mapping intervals. Beam particles are propagated by mapping successively through each interval. We define a 'center' fiducial as a single orbit that continuously threads through all the mapping intervals. This single fiducial is important to maintain as it provides a single reference frame, and hence a reference 'clock' and 'meter stick', for the problem. Each mapping interval is defined by referring to the coordinates of this center fiducial.

For each interval, the beam phase space is partitioned and a set of nominal values of coordinates are selected, one set for each region. These nominal coordinate sets provide the initial values for sub-fiducials and their associated maps to be constructed. The particles in each region are then propagated according to these local maps. The number and method of partitioning is highly dependent upon the physics to be modeled. Partitioning should represent a balance between the order of non-linearity carried by the calculation, the degree of non-linearity present in the external fields and sampled by the beam, and the non-homogeneity of the beam density profile when self-fields are a concern. For example, in a magnetostatic transport region, only a single fiducial and map may need to be calculated. Whereas, in a region with time-dependent rf fields where the wavelength and period of the external fields is comparable to the bunch length and transit time of the beam, then perhaps 10 or more fiducials may be required. When selffield effects are included, the number of partitions may depend upon the non-uniformity of the beam density profile.

\subsection{Longitudinal Beam Dynamics in an RF Cavity}

As an example to illustrate the method, I consider the problem of tracking a bunched beam through an rf cavity. Here, I follow only a single bunch, where the modulation carried by the beam is comparable to the rf period of the cavity, as in the case of an 


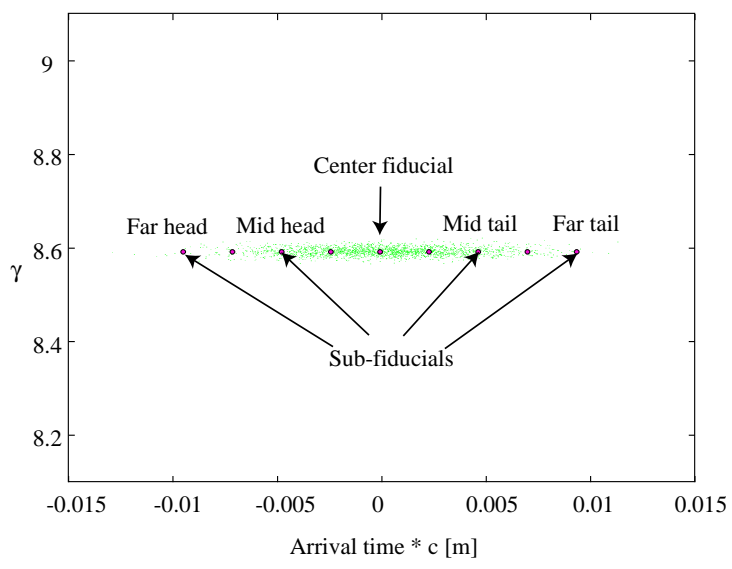

Figure 10.1: Initial fiducial coordinates and beam distribution in longitudinal phase space.

RK-TBA. Also, I will only consider the longitudinal phase space. The center fiducial is placed at the center of the beam distribution, with the sub-fiducials at locations that span the interval in arrival time $(\mathrm{t})$ of the bunch at a given beamline position $(\mathrm{z})$. The initial bunch distribution and fiducial positions is shown in Figure 10.1.

\subsubsection{Fiducial Particle Trajectories}

The fiducial trajectories are calculated using the exact single-particle equations of motion. During transit through the rf cavity field, different fiducials will experience different forces due to arrival time differences. The final coordinates of the various fiducials will generally differ in a non-linear way. The fiducial trajectories in this case are shown in Figure 10.2.

\subsubsection{Comparison of Single Versus Multiple Fiducial Calculation}

The maps induced by the fiducial orbits can, in principle, be calculated to arbitrary order. The accuracy of the mapping for outlying particles is determined by this order parameter. In Figure 10.3 is shown the results of the mapping through the rf cavity for two cases. The first case uses a 4th order Hamiltonian, with a single fiducial (identical to the center fiducial in Figures 10.1 and 10.2). The second case uses a 3rd order Hamiltonian, but with 11 fiducials. In the first case, the combined effect of sampling only low-order field variations and tracking outlying particles leads to inaccuracies. The final distribution suffers additional local energy spread growth, and does not exhibit the 


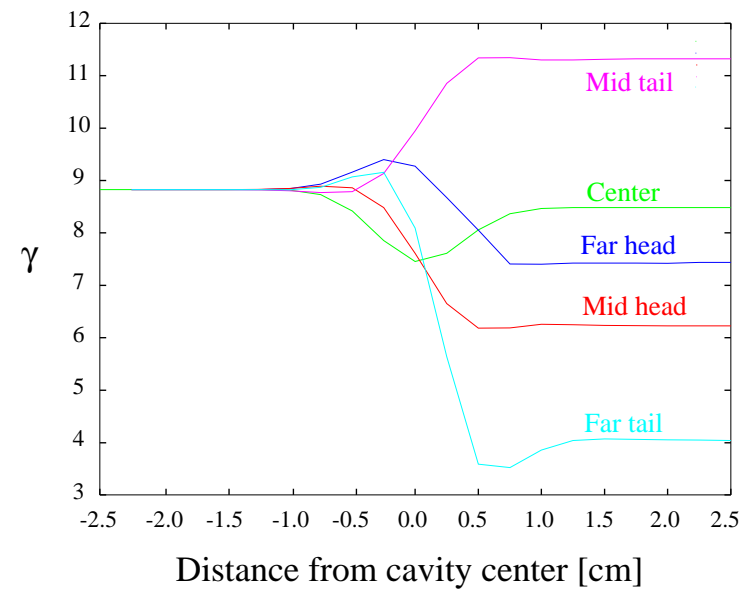

Figure 10.2: Fiducial trajectories in an rf cavity.

appropriate curvature, as seen in the head of the bunch. The second case avoids these problems by sampling the fields at the location of the outlying particles. The effects of errors in the field evaluation and in large coordinate deviations between particles and their associated fiducials are kept small.

\subsection{Inclusion of Self-Field Effects}

\subsubsection{Split Operator Algorithm}

Two separate mappings are applied to the particle phase space coordinates using splitoperator techniques [75] [76] [77] [90]. These techniques are based on splitting the Hamiltonian into pieces that can be solved exactly (or through some desired order of accuracy), and then combining the separate maps to produce an approximate map for the full Hamiltonian. Split-operator symplectic integration algorithms, including the well known leap-frog algorithm of plasma physics simulations [82], are widely used in the treatment of Hamiltonian systems.

The total Hamiltonian is represented in the form

$$
H_{t o t}=H_{k i n}+H_{e x t}+H_{s e l f}
$$

where $H_{k i n}$ is the kinetic portion describing single-particle motion in the absence of all fields, $H_{e x t}$ is the contribution from external fields, and $H_{\text {self }}$ is the contribution from self-fields. The maps from the first two contributions are calculated together, while the 


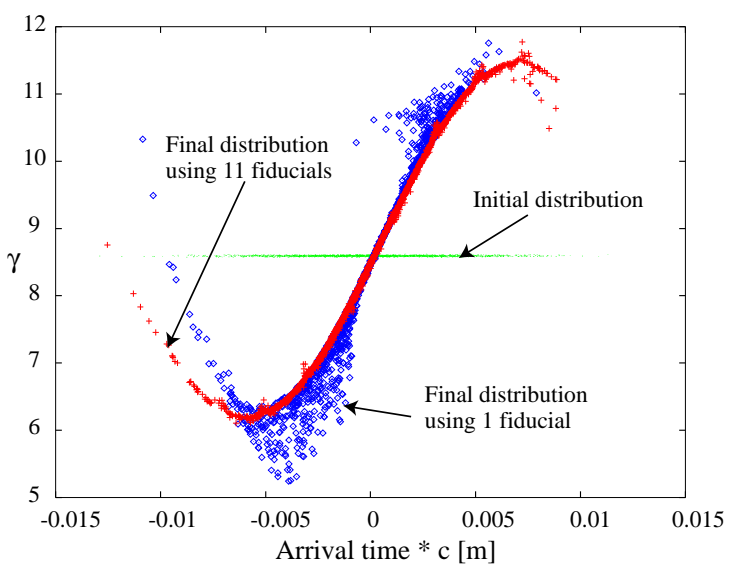

Figure 10.3: Final longitudinal beam distribution under a 3rd order map with multiple fiducials.

map resulting from self-forces is calculated separately. A combined map is then produced to advance particles over an interval $\tau$. Accurate through second order in this step, the dissertationcombined map (6.26) is expressed as

$$
M_{t o t}(\tau)=M_{k i n+e x t}(\tau / 2) \cdot M_{s e l f}(\tau) \cdot M_{k i n+e x t}(\tau / 2) .
$$

The self-fields are determined by numerical solution of Poisson's equation on a 3-D Cartesian grid in the beam's rest frame. Standard techniques are used to solve for the fields at the grid nodes. For accurate representation of the 3-D fields from a bunched beam, we may use grid sizes up to $64 \times 64 \times 128$ nodes, as well as $10^{4}-10^{5}$ macroparticles.

\subsubsection{Changing Representations}

Since we are solving the Maxwell equations at a given instant in time, the particle distribution must be represented at a given moment. However, for the single particle maps, we represent the particles at a given beamline location (z), with a spread in arrival time (t). To faithfully calculate the self-forces, then, we must change the distribution from a 'constant-z' to a 'constant-t' representation.

The multi-fiducial approach again provides some utility. A visual aid to the process of changing representations is provided by the 'world-line' diagram of the fiducials, Figure 10.4. The fiducial trajectories ( $\mathrm{z}$ vs. t) are shown as world-lines. The constant-z beam distribution is shown as black line segments. The first step is to calculate the motion of the sub-fiducials about the center fiducial, and the associated non-linear maps. This 


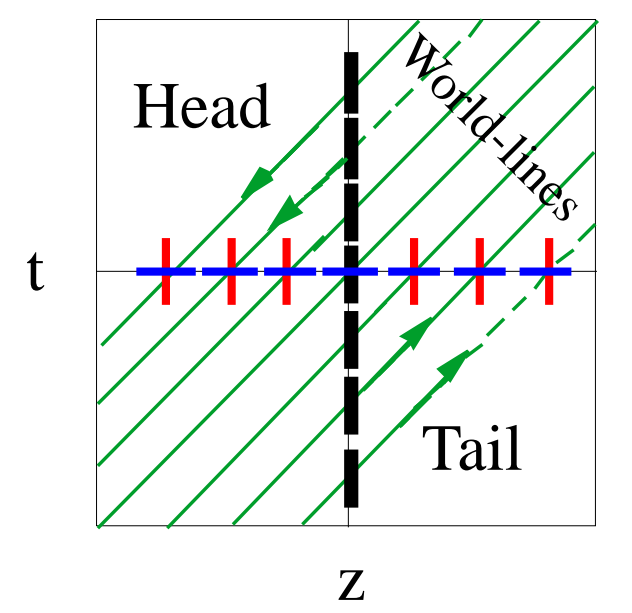

Figure 10.4: Changing beam distribution representation from constant-z to constant-t.

brings all the fiducials to the same time, but at different beamline positions (green lines and arrows). The particles attached to a given sub-fiducial are still spread in time (red line segments). The last step is to apply a linear transformation to all the particles associated with a given fiducial, to bring them to the nominal time (blue line segments). The beam distribution now exhibits a spread in beamline position consistent with observation at a given moment in time.

\subsection{Map Recycling}

Considerable savings in computational costs can be made by re-using maps calculated during the passage of previous bunches across a given beamline interval. However, this is a conditional statement. What is required, at the start of an interval, is that the phase space coordinates of the current and previous fiducials lie close together in an $L_{2}$-measure sense. If this is indeed the case, then the current ensemble of particles can be propagated using the previously calculated maps. It is still necessary to calculate the final coordinates of the current fiducial to provide continuity and stability, and to allow for a slow evolution of parameters, during the simulation. Even so, there is a considerable cost savings in not re-integrating several hundred differential equations per fiducial that are needed to define the maps. 


\section{Chapter 11}

\section{The RKS Code}

\section{$11.1 \quad$ Introduction}

The elements described in the preceding Chapters of this Part have been incorporated into a single simulation code, dubbed 'RKS'. In this Chapter, I describe the RKS code in some detail. Then I will present some simple benchmarking tests to establish the initial credibility of the simulation.

\subsection{Description of the Code}

\subsubsection{Development Path}

The RKS code is an outgrowth of a simple, linear linac simulation code, 'linacxy' [91], that simulates beam transport in a quadrupole FODO channel and includes a simple calculator for space-charge effects. Another simple linac code, 'genmap' [91], provided the basic set of Lie Algebraic, nonlinear map generation subroutines. While these two codes provided a basis for the generation of RKS, all of the routines have been substantially rewitten or reconfigured to handle the increase in modality and complexity required to model additional physics.

The first major change occured in the single-particle trajectory and mapping algorithm. The original codes neglected the possibility of an off-axis excursion of the fiducial orbits. Thus, the maps were built from Hamiltonians (5.10) that only considered on-axis fiducial trajectories. To have the possibility of tracking off-axis trajectories, such as those induced by dipole BBU, it was necessary to first reformulate the mapping generation to accommodate off-axis fiducial coordinates. To do that required an entire re-analysis 
of the power series development used in constructing the deviation Hamiltonians (5.7), (5.8), (5.9). Once accomplished and implemented, this work represents a significant advance for the Lie Algebraic family of tracking codes.

The original space-charge calculator was based upon a free-space Green's function algorithm [83]. It was discoveredearly on in the development, however, that this algorithm used the Green's function based upon the differential Helmholtz operator, when a finite-difference Green's function would have been correct since the Helmholtz operator was based upon gridded quantities and the partial derivatives were replaced by finite-differences. This change was implemented, and the code started producing results that neglected the image charges and currents produced by the beam. While this is a satisfactory approximation for high energy proton beams in large diameter beam tubes, it is insufficient to accurately model high current electron beams in compact geometries. In this latter case, the beam edge will extend to a considerable fraction of the beampipe radius, if not outright fill the tube. Thus, a self-field calculator was needed to take the conducting boundaries into consideration. This was accomplished by turning to the more recently developed multi-grid solvers. I rewrote and re-implemented some of the multi-grid algorithms obtained in the literature to handle curved boundaries in a Cartesian gridding scheme, and added that functionality to the code.

At the same time, I completed the theory and algorithms for the RF sector of the code, and then implemented them into RKS. The implementation was aided by the concurrent development of multi-fiducial tracking. In fact, the multi-fiducial modality of the simulation found its way into all three major components of the code. First, it allows for a lower-order tracking code to accurately handle large-order nonlinearities. Second, it provided a way to map the beam distribution from the z-representation to the t-representation prior to calculating self-field effects. And, third, it provided a means to efficiently calculate the bunch averaged $[\mathrm{R} / \mathrm{Q}]$, and to account for slippage and beam loading effects in the RF interaction.

Map recycling was later added to speed up the simulation when multiple bunches are sent through the linac in sequence. By saving previously calculated maps and the fiducial values, it became possible to stop the simulation during a transient regime calculation, change some parameters, and then restart the calculation in midstream. And once, the simulation entered the steady-state regime, the maps could be saved for later runs with slightly different parameters.

The RKS code itself is comprised of 8400 lines of Fortran 95 code, divided into 13 major program units and 76 subroutines. 


\subsubsection{Code Execution and Major Computational Cycles}

The main elements in the code execution are listed below:

- Read initial conditions, beam parameters, beamline elements.

- Initialize bunch distribution, pre-filled rf cavity fields.

- Loop over individual bunches separated in time.

- Step through the beamline in z. Propagate bunch forward.

- Initialize, then advance rf cavity fields in t.

- Perform diagnostics and output statistics on bunch distribution at different points in $\mathrm{z}$ and $\mathrm{t}$.

\subsection{Transport in Quadrupole Channels}

In this section results will be presented of several sample beam transport simulations in periodic, quadrupole focusing lattices.

\subsubsection{Single Particle Dynamics and Linear Transport Matrices}

The usual first step in designing or analyzing a transport system is to examine the dynamics of single, charged particles in the external fields of this beamline. Several approximations are made initially, and the results are then representative of the lowestorder (linear) beam dynamics. The first assumption is that the longitudinal momentum component of any particle in the beam is much greater than either transverse component of its moment. The second assumption pertains to the external fields, and says that the transverse variation of any field component can have at most a linear dependence on the transverse position coordinates. The Lorentz force equation (8.1) and the NewtonLorentz equation (8.2) can be combined and simplified with these assumptions. Here, we only consider external magnetic fields. For external fields with a (normal) quadrupole symmetry, given by (7.4), the equations of motion can be written in the following form,

$$
\begin{aligned}
& x^{\prime \prime}=\frac{q}{\gamma \beta_{z} m c}\left(-\beta_{z} g x\right)=\frac{|e|}{\gamma m c} g x \\
& y^{\prime \prime}=\frac{q}{\gamma \beta_{z} m c}\left(\beta_{z} g y\right)=-\frac{|e|}{\gamma m c} g y,
\end{aligned}
$$




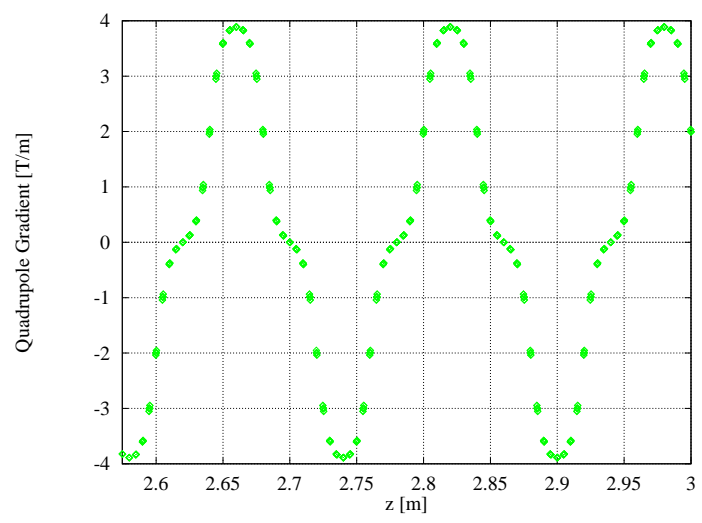

Figure 11.1: Quadrupole gradient along FODO channel.

where the prime $\left({ }^{\prime}\right)$ indicates derivation with respect to the longitudinal coordinate $(z)$, $q=-e$ is the electronic charge, and $g=g(z)$ is the $\mathrm{z}$-dependent quadrupole gradient.

A FODO lattice is a periodic (in $\mathrm{z}$ ) arrangement of focusing (F) and defocusing (D) quadrupoles (where focusing/defocusing refer to one plane, usually the xz-plane, only - the other plane taking on the opposite characteristic), and short drift lengths (O) between them. An example of the quadrupole gradient function, $g(z)$, for a FODO lattice is shown in Figure 11.1.

In the limit of (uncoupled) linear dynamics, the equations of motion (11.1) have the generic form

$$
u^{\prime \prime}+K(z) u=0,
$$

which have the generic solution [80],

$$
\left[\begin{array}{c}
u(z) \\
u^{\prime}(z)
\end{array}\right]=\left[\begin{array}{ll}
C(z) & S(z) \\
C^{\prime}(z) & S^{\prime}(z)
\end{array}\right]\left[\begin{array}{l}
u_{0} \\
u_{0}^{\prime}
\end{array}\right] .
$$

Here, $\mathrm{C}(\mathrm{z})$ and $\mathrm{S}(\mathrm{z})$, are the cosine-like (even) and sine-like (odd) fundamental solutions (or transfer functions) of the equation (11.2). This form is a concrete example of the linear mapping of Section 6.5. For simple focusing lattices (eg. a square-wave FODO lattice), these equations of motion can be solved analytically and expressed in closedform. For lattices that express a significant amount of field fringing, or other complex behavior along the longitudinal direction, the equations of motion must be calculated numerically. For the FODO lattice above, the cosine-like and sine-like functions are shown in Figure 11.2. 
(a)
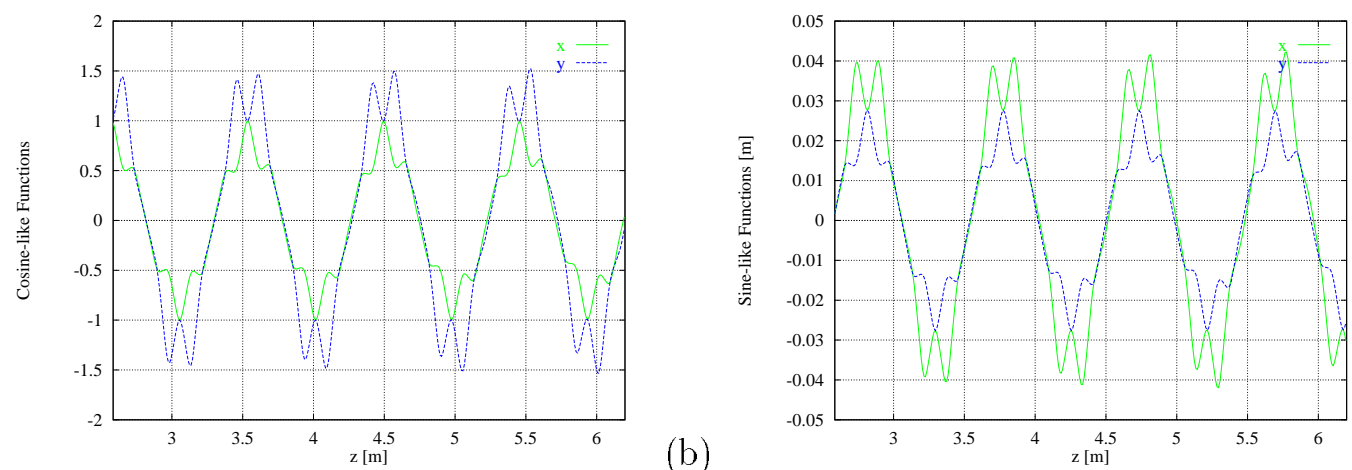

Figure 11.2: Cosine-like (a) and sine-like (b) transfer functions.

\subsubsection{RMS Envelope Equation}

For intense beams, the single particle motion described by (11.1) is altered by the presence of the beam's self-fields. That is, the single particle equation of motion is no longer (11.1), but is more adequately expressed via relations such as

$$
x^{\prime \prime}+K_{x}(z) x-F_{x}^{s}=0,
$$

where $F_{x}^{s}$ is the $(\mathrm{x}-)$ component of the (defocusing) self-field Lorentz force. From the discussion of Section (8.3),

$$
\overrightarrow{F_{\perp}^{s}}=\frac{q \overrightarrow{E_{\perp}^{s}}}{\gamma^{3} m v^{3}}
$$

This self-force component is highly dependent upon the detailed distribution of the ensemble of particles, the position of the individual particle within that ensemble, and the boundary conditions of the external environment (pipe walls, etc.). For studies of transport of whole ensembles of particles, then, another framework is needed. This can be generated from the single particle equations of motion by consideration of the variations in the moments of the transverse beam distributions. Neglecting any coherent dipole motion of the ensemble of particles, the single particle dynamics then affect the quadrupole distribution of the beam. In particular, the 2 nd order moments of the beam distribution follow the equations of motion:

$$
\begin{aligned}
& \frac{d \overline{x^{2}}}{d z}=2 \overline{x x^{\prime}} \\
& \frac{d \overline{x x^{\prime}}}{d z}=\overline{\left(x^{\prime}\right)^{2}}+\overline{x x^{\prime \prime}}=\overline{\left(x^{\prime}\right)^{2}}-K_{x}(z) \overline{x^{2}}+\overline{x F_{x}^{s}}
\end{aligned}
$$




$$
\frac{d \overline{\left(x^{\prime}\right)^{2}}}{d z} \quad=2 \overline{x^{\prime} x^{\prime \prime}} \quad=-2 K_{x}(z) \overline{x x^{\prime}}+2 \overline{x x^{\prime}}+2 \overline{x^{\prime} F_{x}^{s}}
$$

Here, the overbar has been used to denote an ensemble average. The RMS envelope radius is defined as $a=\sqrt{\overline{x^{2}}}$. From the first member of (11.4), we obtain $a a^{\prime}=\overline{x x^{\prime}}$. Differentiating and using the second member of (11.4),

$$
a^{\prime \prime}-\frac{\overline{x^{2}} \overline{\left(x^{\prime}\right)^{2}}-\left(\overline{x x^{\prime}}\right)^{2}}{a^{3}}-\frac{\overline{x x^{\prime \prime}}}{a}=0 .
$$

The (un-normalized) RMS emittance is defined via $\varepsilon_{x}^{2}=\overline{x^{2}\left(x^{\prime}\right)^{2}}-\left(\overline{x x^{\prime}}\right)^{2}$. This quantity is a measure of the beam quality and will vary with beam energy. It is then useful to also define a normalized emittance by $\varepsilon_{N x}=\gamma \beta \varepsilon_{x}$, which becomes an invariant measure in the limit of linear dynamics and self-field forces, even when accelerating fields are present. Using this definition and (11.4), the RMS envelope radius follows the equation of motion

$$
a^{\prime \prime}+K_{x}(z) a-\frac{\varepsilon_{x}^{2}}{a^{3}}-\frac{\overline{x F_{x}^{s}}}{a}=0 .
$$

The emittance enters the equation of motion for the envelope in an analogous way to a nonlinear, repulsive 'pressure' term.

\subsubsection{Linear Self-Field Forces}

The KV uniform transverse beam distribution (8.5) produces a linear variation in position of the electric field (8.6) within the confines of the beam's edge. For an elliptical beam, with semi-axis of length A (B) parallel to the $\mathrm{x}^{-}\left(\mathrm{y}^{-}\right)$axis, the cartesian components of the electric self-fields (neglecting the conducting boundary conditions at the pipe wall), are given by

$$
\begin{aligned}
E_{x}^{s} & =\frac{I}{\pi \epsilon_{0} \beta c(A+B)} \frac{x}{A}, \\
E_{y}^{s} & =\frac{I}{\pi \epsilon_{0} \beta c(A+B)} \frac{y}{B},
\end{aligned}
$$

where I is the beam current. We define a generalized perveance, which measures the beam current and energy in one figure of merit, by

$$
\xi=\frac{q I}{2 \pi \epsilon_{0} m \gamma^{3} v^{3}}
$$


In a uniform density transverse distribution the RMS envelopes are related to the edge radii by $\mathrm{A}=2 \mathrm{a}$ and $\mathrm{B}=2 \mathrm{~b}$. We can then write the envelope equation entirely in terms of the RMS envelopes,

$$
\begin{aligned}
& a^{\prime \prime}+K_{x}(z) a-\frac{\varepsilon_{x}^{2}}{a^{3}}-\frac{\xi}{2(a+b)}=0 \\
& b^{\prime \prime}+K_{y}(z) b-\frac{\varepsilon_{y}^{2}}{b^{3}}-\frac{\xi}{2(a+b)}=0 .
\end{aligned}
$$

Kapchinskij and Vladimirskij [87] originally wrote down this equation for a beam with a particular transverse distribution (8.5). Lapostolle [92] and Sacherer [93] independently

wrote down these RMS equations, and went further to show these are also valid to describe the envelope dynamics of any elliptical beam with the same perveance and set of 2 nd order moments, regardless of the details of the transverse distribution. This is an important step in that we can now use apply this concept of equivalent beams to apply the results of our linear analysis across different models of the beam distribution.

\subsubsection{Quadrupole FODO Channel}

A simulation of beam dynamics in a uniform quadrupole FODO channel is presented here. The FODO lattice has a $16 \mathrm{~cm}$ period, with a $4 \mathrm{~T} / \mathrm{m}$ peak gradient. The quadrupole gradient field and the single-particle transfer functions are shown in Figures 11.1 and 11.2, respectively. The beam used in the simulation is composed of 8192 macroparticles, carrying 600 A current, with $250 \pi \mathrm{mm}$ mrad normalized RMS emittances in both horizontal and vertical phase spaces.

\section{Matched 2D KV Beam}

In the first case we consider a uniform (in z) beam with zero spread in energy, and a 2D transverse KV distribution. The envelope and emittance evolution is shown in Figure 11.3. The beam envelopes oscillate with a small, constant amplitude $(\sim 0.7 \mathrm{~mm})$ about an average radius $(\sim 2.9 \mathrm{~mm})$. The assumption of constant emittance, implicit within the envelope equation, is approximately borne out by the simulation. The slight increase is emittance, and in the energy spread (Figure 11.4), is due to the ever-present noise in the self-field mapping algorithm interacting with a singular beam distribution function. 
(a)

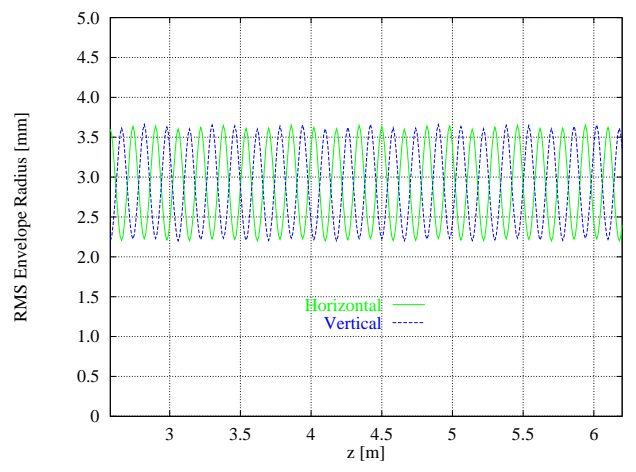

(b)

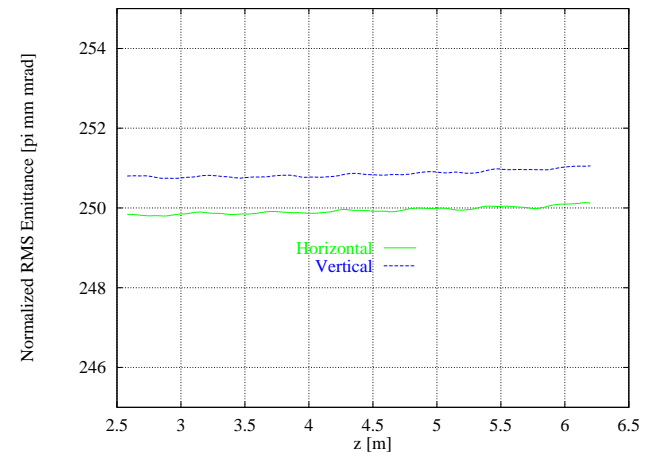

Figure 11.3: RMS envelopes (a) and normalized emittance (b) evolution of a matched $\mathrm{KV}$ beam in a quadrupole FODO channel.

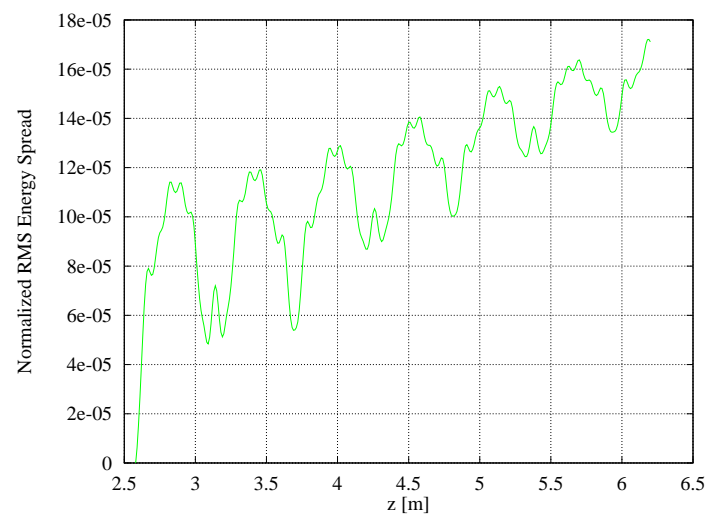

Figure 11.4: Spurious growth in energy spread. 


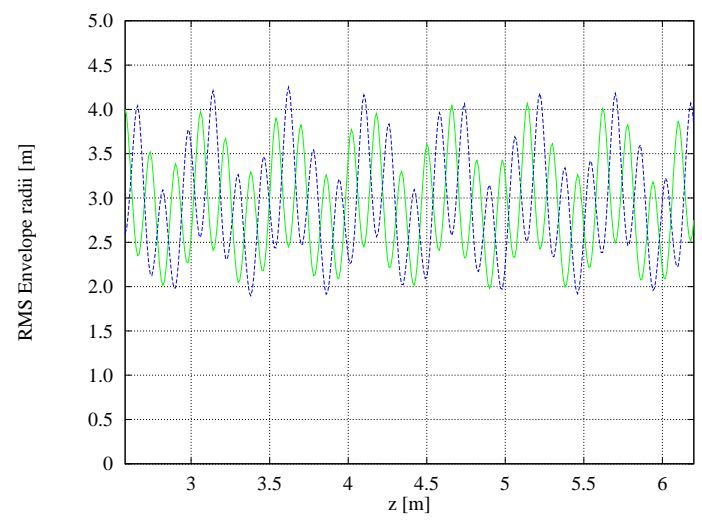

Figure 11.5: RMS envelopes of a mismatched KV beam in a quadrupole FODO channel.

\section{Mismatched KV Beam}

The effect of a mismatch is shown in Figure 11.5. The beam again carries $600 \mathrm{~A}$, with a $250 \pi \mathrm{mm}$ mrad RMS normalized emittance. In this case, however, the beam was injected into the channel with a mismatch in the average envelope radius $(3.3 \mathrm{~mm}$ instead of $2.9 \mathrm{~mm}$ ). As can be seen, the effect of such a mismatch is to induce a collective ('breathing') mode of oscillation of the beam envelopes about the average radius. This is a small perturbation, however, and does not lead to any new sources of emittance growth. It is, then, a stable oscillation.

\section{Matched 3D Semigaussian Beam}

In the last example of this section, simulations of an axially bunched beam are presented. This bunching can be due to a longitudinal modulation of the line density in an otherwise axially uniform beam. Hence, the simulation examines the dynamics of a short slice of a much longer beam - the slice length equal to the modulation period $(2.624 \mathrm{~cm}$ here, for an $11.424 \mathrm{GHz}$ modulation frequency on a velocity of light beam.) The $6 \mathrm{D}$ phase space distribution is taken to be the more realistic 'semigaussian'. The transverse position coordinates of the macroparticles are uniformly distributed across the edge envelope of the beam; the transverse momenta and particle energy are chosen from a normal distribution. For a bunched beam, the longitudinal position coordinates are also chosen from a normal distribution; in an unbunched beam, these coordinates may then be chosen from a uniform distribution.

The dynamics of bunched beams shows more interesting behavior since the self-fields no longer exhibit axial symmetry. The fringing of these fields at the head and tail 
(a)
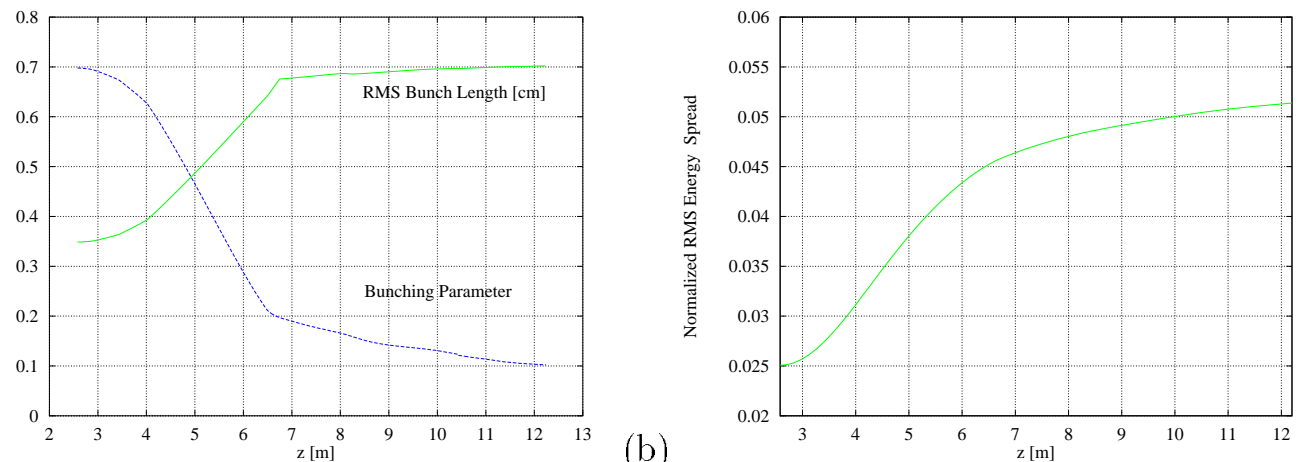

Figure 11.6: Evolution of beam modulation (a) and energy spread (b).

of a bunch then induces a spread in the particle energy correlated with axial position. Hence, particles at the head will gain energy at the expense of particles in the tail. In the absence of external accelerating fields, the average energy of the bunch remains constant in the transport channel, while the variation in energy increases to an asymptotic value. Without axial focusing, an initially bunched beam will expand in longitudinal extent as the energy spread increases. This is shown in Figure 11.6.

The effect of this longitudinal dynamic upon the transverse dynamics is quite readily deduced. An initially tightly bunched beam, carrying a given current, will generate stronger transverse self-field forces than that of an axially uniform beam with the same current. This is just due to the higher charge densities present near the center of the bunch. This effect is also exacerbated for relativistic beams by a factor of $\gamma$. The selffield defocusing force is thus stronger in bunched beams than in unbunched beams, and this affects the envelope dynamics (11.5).

A bunched beam that is transversely matched into a periodic transport channel will have a larger average radius than an unbunched beam. In a transport line without any axial focusing, the $6 \mathrm{D}$ beam distribution will seek a new equilibrium state. During this process of axial expansion and heating, the local charge density gradually decreases. If this occurs over many periods of the transverse focusing lattice, this process is adiabatic, and the transverse dynamics can maintain pseudo-equilibrium. The net effect, shown in Figure 11.7, is that as the initially bunched beam evolves towards an axially uniform beam, the average radius decreases towards that asymptotic value for a uniform beam. The adiabaticity of this process is indicated by observing that the transverse emittances show only a small variation over many lattice periods.

This simulation demonstrates the utility of the concept of equivalent beams. Casting 
(a)

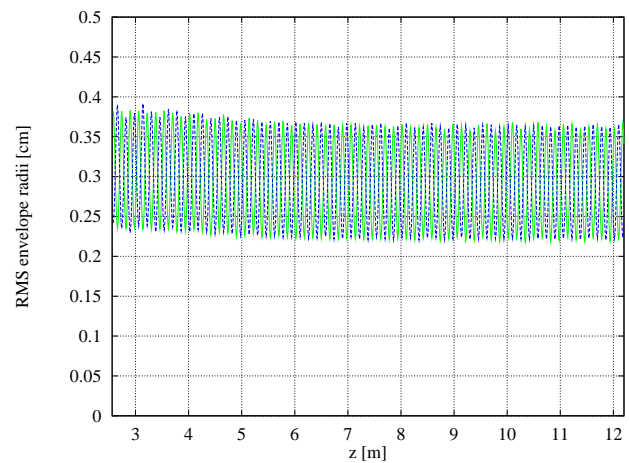

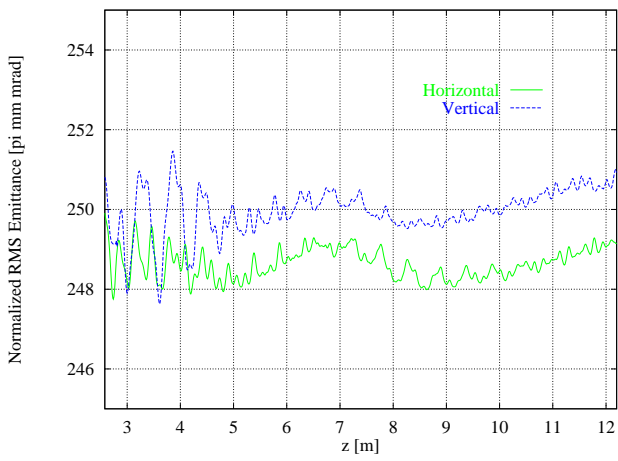

Figure 11.7: RMS envelopes and emittance of an initially bunched beam.

\begin{tabular}{|c|c|c|c|c|}
\hline Mode frequency [GHz] & 11.720 & & Beam energy [MeV] & 2.50 \\
\hline \hline$[\mathrm{R} / \mathrm{Q}][\Omega]$ & 17.2 & & Peak beam current $[\mathrm{A}]$ & 600 \\
\hline Qwall & 4800 & & Current risetime $[\mathrm{ns}]$ & 20 \\
\hline Qext & 50 & & Modulation frequency [GHz] & 11.424 \\
\hline Tuning angle $[\mathrm{rad}]$ & 1.2 & & Bunching parameter & 0.81 \\
\hline
\end{tabular}

Table 11.1: Beam and cavity parameters.

aside small differences due to the finite spread of energy and momenta (which induces a spread in betatron wavenumbers among the beam particles) in the semigaussian model compared to the KV model, the 'equilibrated' 3D beam displays the same envelope parameters as the $2 \mathrm{D}$ beam.

\subsection{Design of Idler and Output Cavities}

The collection of idler and output cavities in an RK-TBA requires detailed design so that its rf properties match the demands of the device in terms of beam transport stability and output power production. In particular, the resonant frequency and loaded Q-value of a cavity determine the longitudinal impedance seen by the beam. This impedance is tuned inductively to counteract the (capacitive) effects of space charge debunching and loss of rf current carried by the beam. This is true for both idler and output structures. In addition, the external Q-value of the output structures must be matched to the output rf power production levels required. There is no input power in these structures.

Here, I present a short simulation of the beam-cavity interaction. The relevant parameters for the cavity and the beam are listed in Table 11.1. This simulation tracks the transient evolution of the cavity amplitude and phase, as well as the energy modulation 
(a)

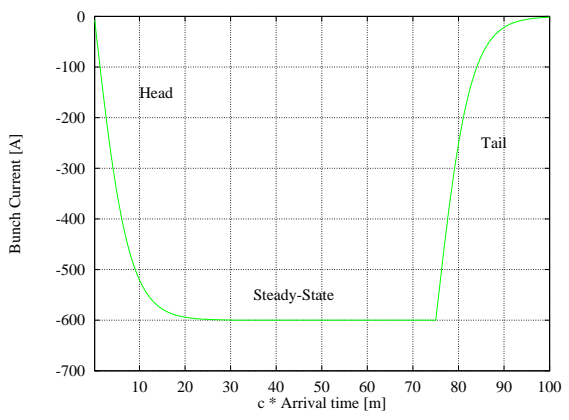

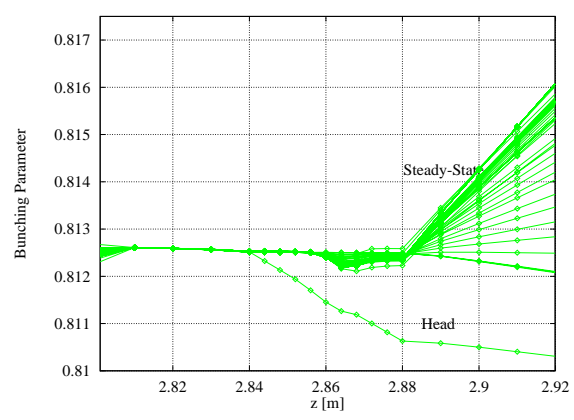

Figure 11.8: Time evolution of (a) DC bunch current and (b) bunching parameter as a function of propagation distance.

and power loss in the beam, from the head to the body of the pulse. The time evolution of the current pulse is shown in Figure 11.8, along with the evolution of the bunching parameter describing the depth of modulation carried by the individual beam bunches. In this second plot, the different traces correspond to different bunches along the beam (from the head to the main body of the pulse), thus resolving the interaction in time as well as a function of propagation distance. For reference, the cavity is centered at $\mathrm{z}=2.86 \mathrm{~m}$, and the interaction with the beam occurs over a length of $\sim 4 \mathrm{~cm}$.

\subsubsection{Steady-State Scaling}

In this section, we consider the steady-state behavior of the beam-cavity system. We neglect the energy spread of the bunch as it passes through the cavity, and further assume that the individual particle velocities remain constant (the 'linac' approximation). The arrival time at the center of the cavity of a given particle is given by $t(z)=t_{0}+(z-$ $\left.z_{0}\right) /\left(\beta_{0} c\right)+\hat{s} / c$. The distribution in arrival time $(\widehat{s})$ is taken to be gaussian. Following the phase convention of $(9.9)$, we set $t_{0}=0$. The transverse distribution is also taken to be gaussian, and essentially static as the bunch traverses the cavity. We take the bunch distribution to be

$$
g\left(\overrightarrow{r_{\perp}}, \widehat{s}\right)=\frac{\exp \left(-\frac{r_{\perp}^{2}}{2 \sigma_{r}^{2}}\right)}{2 \pi \sigma_{r}^{2}} \frac{\exp \left(-\frac{\widehat{s}^{2}}{2 \sigma_{s}^{2}}\right)}{\sqrt{2 \pi} \sigma_{s}} .
$$

The rf mode amplitude of an idler or output structure is given by

$$
a_{\lambda}(t) \cong \widehat{a_{\lambda}}(t) \cos \left(\omega_{b} t+\varphi_{\lambda}(t)\right),
$$


where

$$
\begin{gathered}
\widehat{a}(t)=\left|\frac{\widetilde{V_{c}}}{\widetilde{w_{0}}}\right|=R_{\lambda}\left|I_{b}\right| \frac{F_{b}}{\widetilde{w_{0}}} \mid \cos \psi, \\
\varphi_{\lambda}(t)=-\angle\left(\frac{\widetilde{V_{c}}}{\widetilde{w_{0}}}\right)=\psi-\angle\left(\frac{F_{b}}{\widetilde{w_{0}}}\right)=\psi+\varphi_{b} .
\end{gathered}
$$

Calculation of the mode transit function is a straightforward gaussian integral. Under the 'linac' approximation, it is easily shown ${ }^{1}$ using $(9.6,9.20,9.21)$ that

$$
\widetilde{w}=\widetilde{w_{0}} \exp \left(-i k_{b} \widehat{s}\right)=N_{\lambda} \exp \left(-\frac{k_{b}^{2} \sigma^{2}}{2 \beta_{0}^{2}}\right) \exp \left(-i k_{b} \widehat{s}\right)
$$

where $k_{b}=\omega_{b} / c$. And, in this case, the bunch-averaged form factor is real-valued,

$$
F_{b}=\exp \left(-\frac{k_{b}^{2} \sigma_{s}^{2}}{2}\right)
$$

The mode amplitude and phase follow as

$$
\begin{aligned}
\widehat{a} & =\frac{R_{\lambda}\left|I_{b}\right|}{\sqrt{\frac{1}{2} \epsilon_{0} \omega_{\lambda}\left[\frac{R}{Q}\right]_{\lambda}}} \cos \psi \exp \left(-\frac{1}{2} k_{b}^{2} \sigma_{s}^{2}\right), \\
\varphi_{\lambda} & =\psi+k_{b} \widehat{s} .
\end{aligned}
$$

Following the analysis in Section 3, we can find the steady-state power flow out of the beam and into the wall and into the reverse waveguide mode,

$$
\begin{aligned}
\bar{P}_{b} & =R_{\lambda} I_{b}^{2} \cos ^{2} \psi \exp \left(-k_{b}^{2} \sigma_{s}^{2}\right) \\
\bar{P}_{w} & =\frac{Q_{L}}{Q_{w}} R_{\lambda} I_{b}^{2} \cos ^{2} \psi \exp \left(-k_{b}^{2} \sigma_{s}^{2}\right) \\
\bar{P}_{-} & =\frac{Q_{L}}{Q_{e x t}} R_{\lambda} I_{b}^{2} \cos ^{2} \psi \exp \left(-k_{b}^{2} \sigma_{s}^{2}\right) .
\end{aligned}
$$

The time evolution of the output power and phase is shown in Figure 11.9. The steady-state value of the power agrees with the simple analytic estimate (11.7).

\footnotetext{
${ }^{1}$ The higher-order terms in (9.21), (9.22) are identically zero for constant velocity particles. This is consistent with the Panofsky-Wenzel theorem [94].
} 
(a)

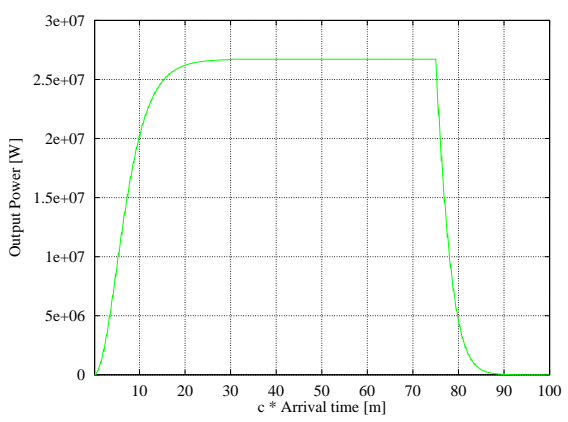

(b)

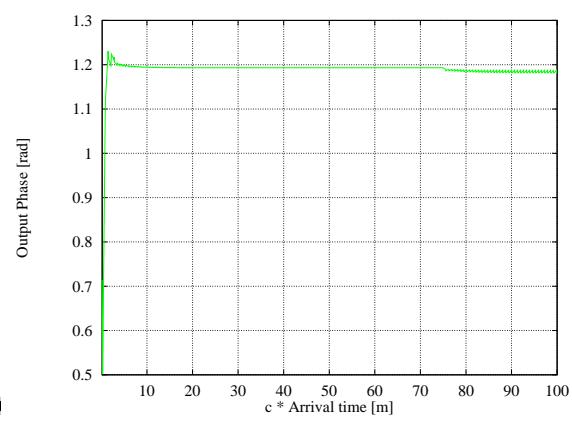

Figure 11.9: Time evolution of (a) output power and (b) phase.

\subsubsection{Beam Energy Modulation}

The beam energy distribution undergoes modulation from this field. The real voltage change seen by a beam particle is given by

$$
\triangle V\left(\overrightarrow{r_{\perp}}, \widehat{s}\right)=-\int_{\text {cavity }} d z E_{z}\left(\overrightarrow{r_{\perp}}, s, z\right)
$$

Carrying out this integral gives

$$
\triangle V\left(\overrightarrow{r_{\perp}}, \widehat{s}\right)=-R_{\lambda}\left|I_{b}\right| \exp \left(-\frac{k_{b}^{2} \sigma_{s}^{2}}{2}\right) \cos \psi \cos \left(\psi+k_{b} \widehat{s}\right) .
$$

The modulation of beam energy $(\gamma)$ is then

$$
\triangle \gamma(\widehat{s})=-\frac{R_{\lambda}\left|I_{b}\right|}{m_{e} c^{2} /|e|} \exp \left(-\frac{k_{b}^{2} \sigma_{s}^{2}}{2}\right) \cos \psi \cos \left(\psi+k_{b} \widehat{s}\right) .
$$

The cavity interaction thus produces a correlation between arrival time and energy. The variation of the average energy of the bunch along the beamline, and development of the intra-bunch energy spread, is shown in Figure 11.10. Again, the different traces in the plots correspond to different bunches along the beam, from the head to the main body of the pulse, and resolve the interaction in time. The bunches in the main body of the pulse interact more strongly with the cavity than those bunches in the beam head, since the cavity mode amplitude is still increasing to its equilibrium value. We see this in the figure when the later bunches both lose more energy and gain more energy spread than the earlier bunches. 
(a)

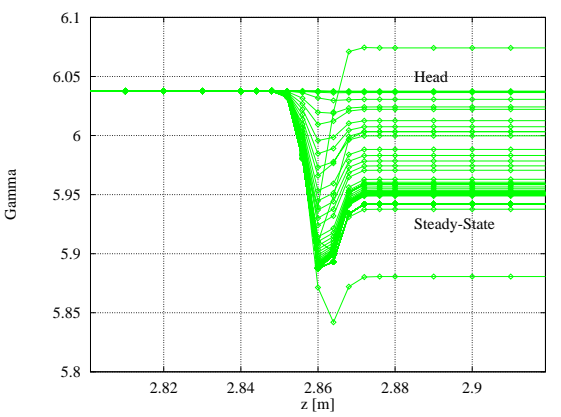

(b)

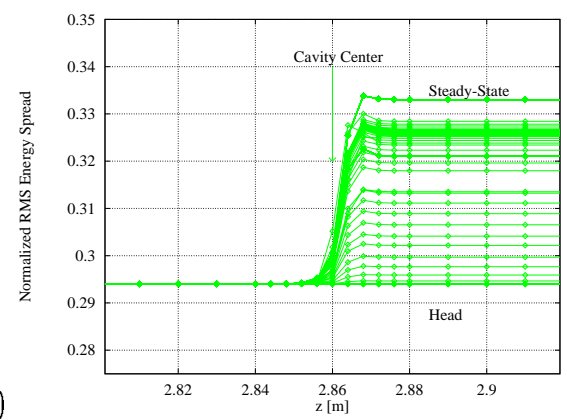

Figure 11.10: Evolution of (a) average bunch energy and (b) energy spread.

\subsubsection{Inductive Detuning for Beam Stability}

The energy modulation imposed upon the beam by its interaction with the cavity, (11.8), contains a sinusoidal variation with arrival time and a phase offset determined by the cavity tuning angle. The tuning angle (9.16) contains two free parameters, $\omega_{b}$ and $Q_{L}$. For RK-TBA applications, these parameters are used to adjust the amount of energy loss experienced between the head and tail of the rf bunch. Or, equivalently, the average bunch energy loss and the longitudinal phase-space rotation of the bunch. The average energy loss is determined by the rf power required, while the bunch rotation degree of freedom is used to counterbalance the opposite rotation (and debunching effect) of self-fields. The average energy change for the bunch is then found to be

$$
\overline{\Delta \gamma}=-\frac{\bar{P}_{b}}{V_{0}\left|I_{b}\right|}=-\frac{\left(Q_{e x t} / Q_{L}\right) \bar{P}_{-}}{V_{0}\left|I_{b}\right|}
$$

where $V_{0}=m c^{2} / e$ is the electronic rest mass in voltage units. 


\section{Part III}

\section{Studies Of Beam Dynamics In RK-TBAs}



It's the same old story. There's no such thing as a stable beam in an rf linac.

- R. Ruth, 11 December 1998

All stable processes we shall predict. All unstable processes we shall control.

— J. Von Neumann 


\section{Chapter 12}

\section{Design of the RTA Experiment}

\subsection{Introduction}

In this Chapter is presented the results of a detailed study of beam dynamics and power production in a prototype RKTBA. This design is presented as a candidate beamline for the RTA project, in a lower energy and current configuration. The entire RTA experiment beamline is shown in Figure 12.1. In this Chapter I present the design and supporting simulations which describe the layout and beam dynamics in the modulation section (SL4 Klystron) through $10 \mathrm{~m}$ of the Main TBA section. A detailed schematic of the SL4 and the beginning of the Main TBA is shown in Figure 12.2, displaying the focusing elements, re-acceleration modules, and the first nine rf cavities. The remaining portion of the beamline used in the simulation is a continuation of the periodic main TBA section shown.

The injector and accelerator are assumed to deliver a $2 \mathrm{MeV}, 1.0 \mathrm{kA}$ beam with an $800 \pi \mathrm{mm}$-mrad normalized edge emittance. Over the beam pulse, the beam energy is taken to be constant while the current rises from 0 A to to its steady-state value of 1000 A, with a 100 ns risetime and 150 ns flat-top.

\subsection{Description of the RTA Beamline}

\subsubsection{SL4 Klystron Modulator}

The modulation section of the RTA is the input and gain cavities of a previously designed and tested relativistic klystron, the SL4 [40]. Figure 12.3 shows a schematic of the gain section of the klystron modulator. The cavity properties are shown in Table 12.1. The 


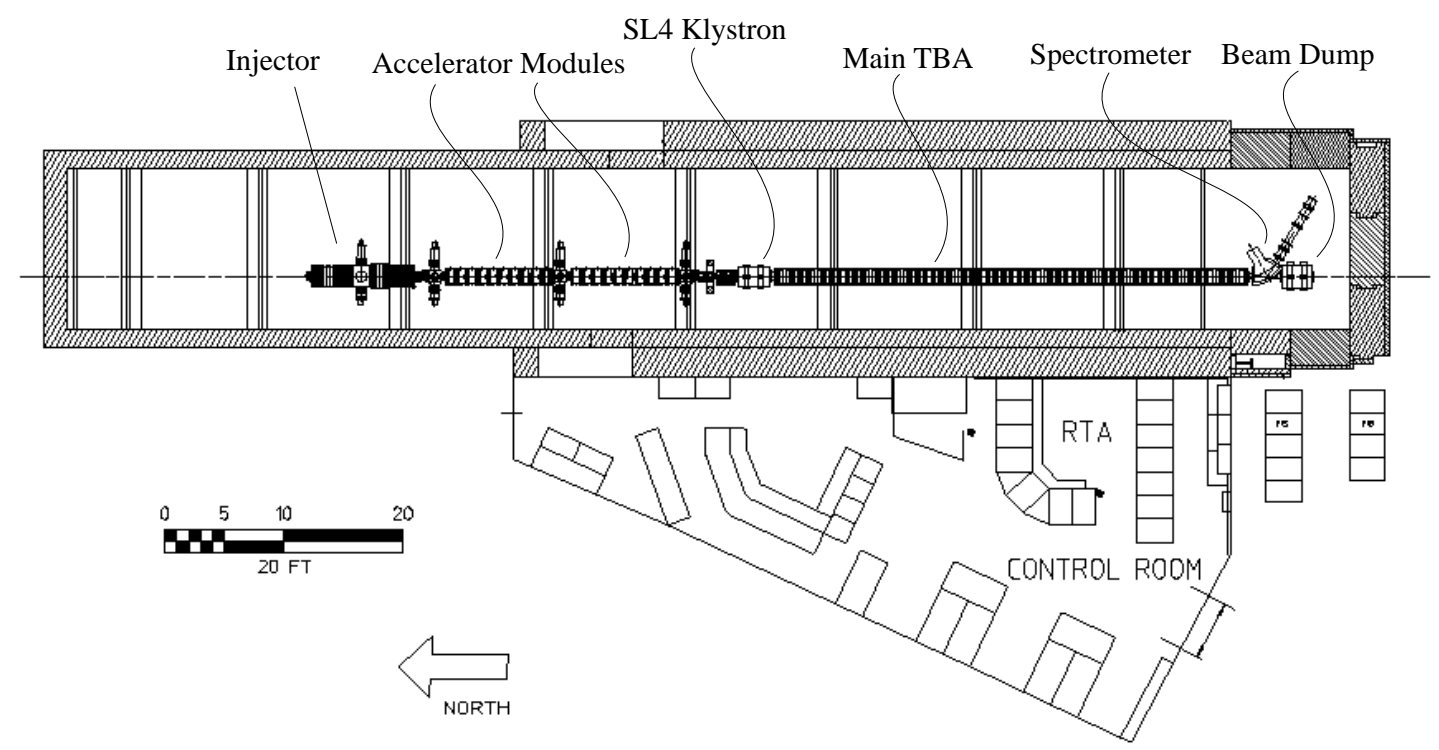

Figure 12.1: RTA experiment layout.

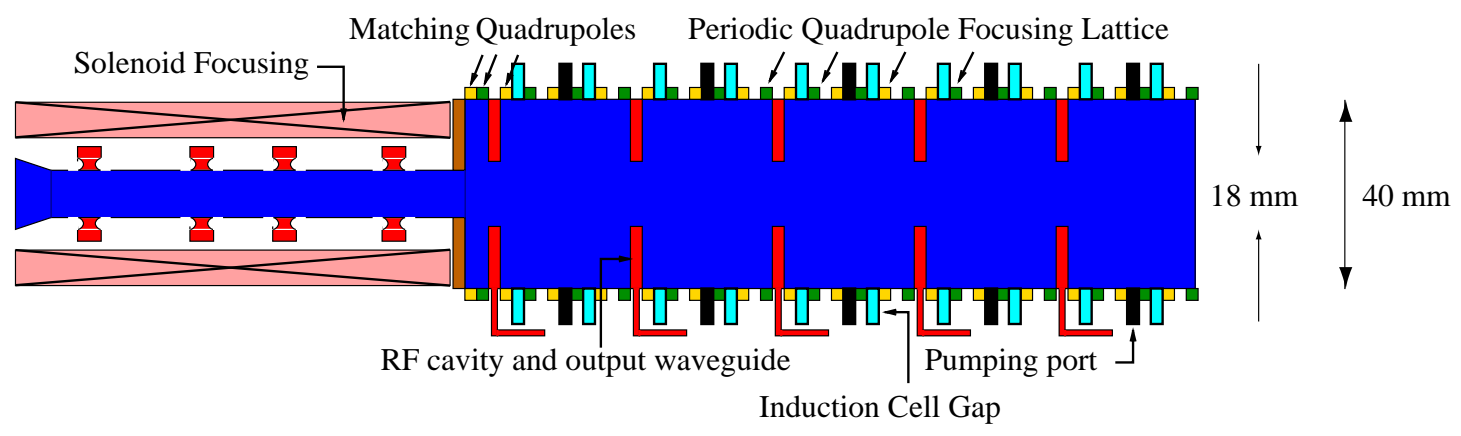

Figure 12.2: Modulation and extraction section in RTA.

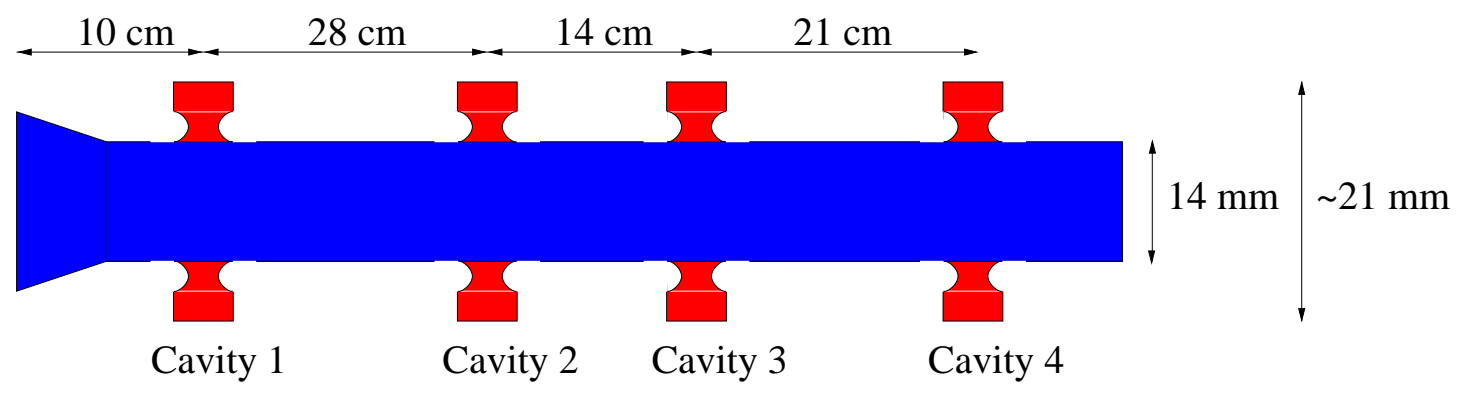

Figure 12.3: SL4 klystron gain cavities. 


\begin{tabular}{|c|c|c|c|c|}
\hline Cavity & 1 & 2 & 3 & 4 \\
\hline \hline Frequency $[\mathrm{GHz}]$ & 11.428 & 11.393 & 11.447 & 11.469 \\
\hline$[\mathrm{R} / \mathrm{Q}][\Omega]$ & 60 & 60 & 60 & 60 \\
\hline Qext & 320 & 120 & 120 & 120 \\
\hline Qwall & 4800 & 4800 & 4800 & 4800 \\
\hline \hline Input Power $[\mathrm{kW}]$ & 1.0 & 0 & 0 & 0 \\
\hline
\end{tabular}

Table 12.1: SL4 cavity parameters.

\begin{tabular}{|c|c|c|}
\hline Cavity & $5-6$ & $7-40$ \\
\hline \hline Frequency [GHz] & 11.84 & 11.72 \\
\hline$[\mathrm{R} / \mathrm{Q}][\Omega]$ & 31.6 & 17.2 \\
\hline Qext & 50 & 50 \\
\hline Qwall & 4800 & 4800 \\
\hline
\end{tabular}

Table 12.2: Design Parameters of Main TBA Cavitites.

first (input) cavity accepts rf power from a low power $(<5 \mathrm{~kW}), \mathrm{X}$-band, travelling-wave tube (TWT) or magnetron source, operating at a nominal frequency of $11.424 \mathrm{GHz}$. The beam enters from the left in the schematic $(\mathrm{z}=0 \mathrm{~m})$. The input $\mathrm{rf}$ power modulates the beam energy in Cavity 1 and initiates the bunching process. The following cavities are then driven by the rf component of the beam current, which develops in the drift spaces between the cavities.

The beam focusing in this section is performed by a set of 4 independent solenoid magnets (shown as a single solenoid in Figure 12.2). The solenoids have an inner radius of $9.6 \mathrm{~cm}$, an outer radius of $12.8 \mathrm{~cm}$, and a length of $15.5 \mathrm{~cm}$. A magnetic field clamp (shunt) is placed at the exit plane of the SL4 $(\mathrm{z}=0.98 \mathrm{~m})$ to contain and reflect the solenoid field.

\subsubsection{Main TBA Section}

A schematic of a single period of the main extraction section is shown in Figure 12.4. Each module in the lattice holds one output rf cavity with dual output waveguides, six focusing and defocusing quadrupoles, and two complete induction re-acceleration cells with gaps. The rf properties of the modules are shown in Table 12.2. The first cavity in the main TBA lattice is Cavity \#5. The first two cavities are detuned by 1.3 radians from resonance to provide additional bunch rotation. All following cavities are detuned by 1.2 radians. The cavities are designed to produce $50 \mathrm{MW}$ of rf power from a beam modulated at $11.424 \mathrm{GHz}$ with a bunching factor of 0.70 and carrying $950 \mathrm{~A}$ of DC 


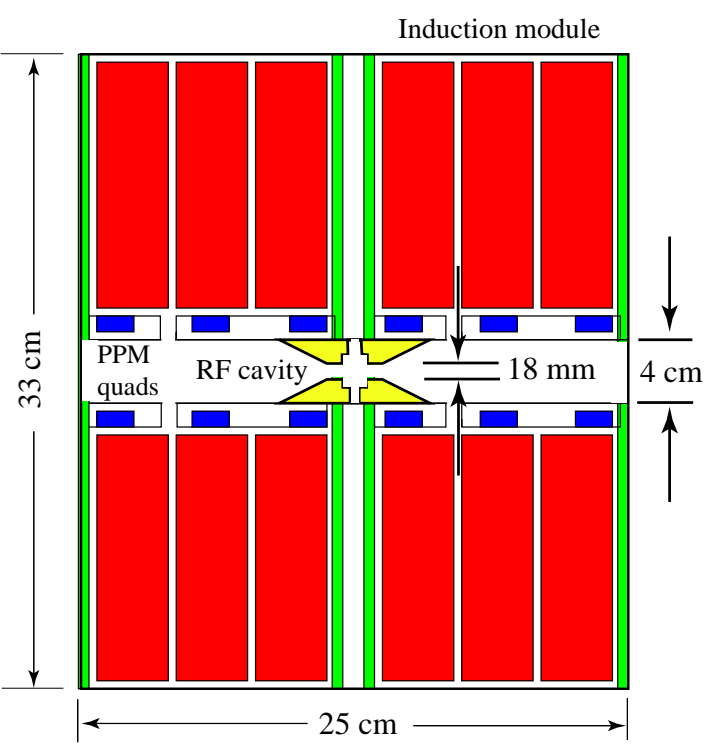

Figure 12.4: One period of the main RTA extraction section.

current. The induction modules are then required to replace this lost power, and supply $26.25 \mathrm{kV}$ across each of the two gaps in each module.

The transverse focusing is accomplished by the quadrupole magnets. We have chosen to separate the rf cavities by $1 / 2$ of a betatron wavelength. There are six quadrupoles in each TBA period, making 3 complete FODO cells. The betatron phase advance per FODO cell is 60 degrees, so that the net phase advance between rf cavities is 180 degrees. To achieve this, the permanent magnets must produce a $14 \mathrm{~T} / \mathrm{m}$ quadrupole gradient. With a $4 \mathrm{~cm}$ beam pipe diameter, the magnet blocks must have a remanent field of 2800 G. While high, this is well within the field strengths achievable with ferrites.

\subsection{Simulation Parameters}

We have modeled the beam dynamics and the evolution of the cavity fields from initial transients into the steady-state regime. The main parameters of the simulation are given in Table 12.3. In this simulation 8192 macroparticles were used, and initally loaded into a 3D semi-gaussian distribution ${ }^{1}$. The initial normalized RMS emittance is taken to be $250 \pi \mathrm{mm}-\mathrm{mrad}$ (1000 $\pi \mathrm{mm}$-mrad normalized edge emittance). The rf and induction cavity field profile distributions in z were taken to be gaussian with standard deviations

\footnotetext{
${ }^{1}$ The particles have a Maxwellian distribution in momentum and energy spread, uniform distribution in transverse position and arrival time.
} 


\begin{tabular}{|c|c|}
\hline Nominal beam energy [MeV] & $2.5-3.0$ \\
\hline \hline DC Beam current [A] & $930-1000$ \\
\hline$\epsilon_{\text {edge }}$, normalized $[\pi \mathrm{mm} \mathrm{mr}]$ & $1000-1200$ \\
\hline Bunching parameter & $0.5-0.8$ \\
\hline FODO cell length & $25 / 3 \mathrm{~cm}$ \\
\hline Phase advance/cell & $60 \mathrm{deg}$. \\
\hline RF cavity spacing & $25 \mathrm{~cm}$ \\
\hline RF output power $[\mathrm{MW}]$ & $25-60$ \\
\hline Induction gap voltage $[\mathrm{kV}]$ & 26.25 \\
\hline Modulation frequency $[\mathrm{GHz}]$ & 11.424 \\
\hline Rf cavity frequency $[\mathrm{GHz}]$ & $11.84,11.72$ \\
\hline$Q_{\text {loaded }}$ & 50 \\
\hline$[\mathrm{R} / \mathrm{Q}][\Omega]$ & $31.6,17.2$ \\
\hline Total length & $10 \mathrm{~m}$ \\
\hline
\end{tabular}

Table 12.3: Main TBA parameters.

of $4.03 \mathrm{~mm}$ and $5.0 \mathrm{~mm}$, respectively.

\subsection{Beam Matching from a Solenoid to a Quadrupole Chan- nel}

The matching of the beam from a solenoidal to a periodic quadrupole transport channel poses one of the major difficulties in a low-energy TBA. There is tremendous potential for mismatches to occur, and to result in large increases in the transverse emittance and/or major loss of current. The procedure used in these simulations to perform this matching was to proceed backwards. For a given beam energy, current and emittance, betatron period and FODO cell lengths, the RMS envelopes in an infinitely-long quadrupole transport channel can be found fairly quickly from a combination of analysis and trialand-error simulation. Once this is done, the beam distribution at the points in the FODO lattice where the beam is round (ie. $\sigma_{x}=\sigma_{y}$ ) is located. This becomes the equilibrium round beam distribution in the solenoid channel.

The matching between these two is performed by introducing an extra magnet into the quadrupole lattice. The quadrupole gradient of this magnet (and possibly others) is adjusted so that the matched beam in the FODO lattice is focused to a round beam. At this point the quadrupole lattice ends and the solenoid channel begins. The solenoid field strength is adjusted so that the initial beam distribution is approximately in equilibrium. 
(a)

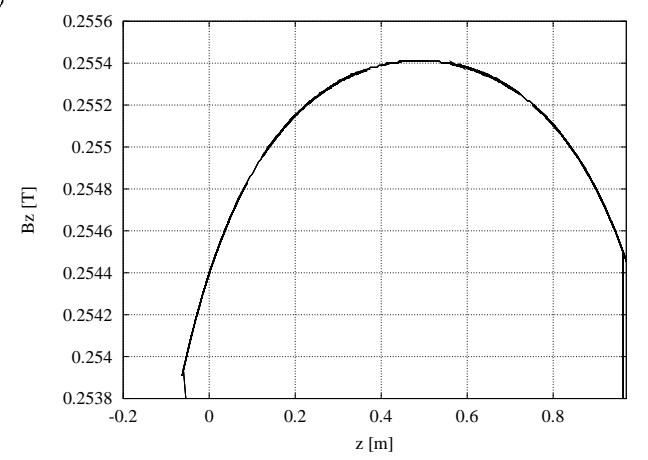

(b)

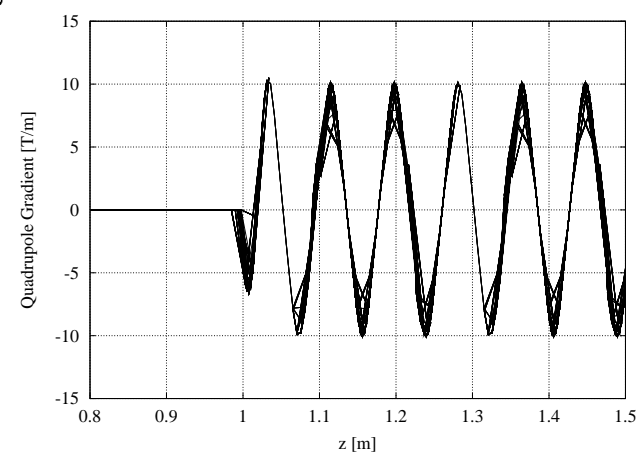

Figure 12.5: Solenoid (a) and Quadrupole (b) fields in the matching section.

Once this matching is performed, the simulation is run forwards again. The matched round beam distribution is loaded into the solenoid channel and allowed to propagate forward to the matching region and beyond into the quadrupole lattice.

\subsubsection{Fields and Envelopes}

The fields in the matching region are shown in Figure 12.5. The solenoids around the SL4 are driven to produce a field with an average value of $2550 \mathrm{G}$, from the start of the beamline at $\mathrm{z}=-0.06 \mathrm{~m}$ to the magnetic shunt at $\mathrm{z}=0.98 \mathrm{~m}$. The quadrupole focusing lattice then begins. The first and second quadrupole magnets have surface fields that are $80 \%$ and $116 \%$ of the nominal value, respectively. The first two quadrupole magnets abut each other. After that there are the usual drift spaces between quadrupoles in a FODO lattice. The matching from solenoid to quadrupole lattices is performed by these first two quadrupoles, which take an initially round beam and quickly introduce a quadrupole moment to the transverse distribution.

The RMS envelopes in the matching section are shown in Figure 12.6, where the horizontal (vertical) envelopes are shown with red/solid (green/dashed) lines. The simulation actually begins at $\mathrm{z}=-0.06 \mathrm{~m}$ for appropriate matching of the envelope parameters. At this point in the beamline, the beam envelopes (x, y) have RMS values of $2.40 \mathrm{~mm}$, while the normalized momentum distribution has an RMS value of $104 \mathrm{mrad}$. Hence, the initial normalized RMS emittance of $250 \pi \mathrm{mm}$-mrad.

\subsubsection{Transverse Phase-Space Evolution}

The transverse phase-space evolution of the bunches in the matching section are shown in Figure 12.8, where the two colors differentiate the vertical and horizontal phase space 


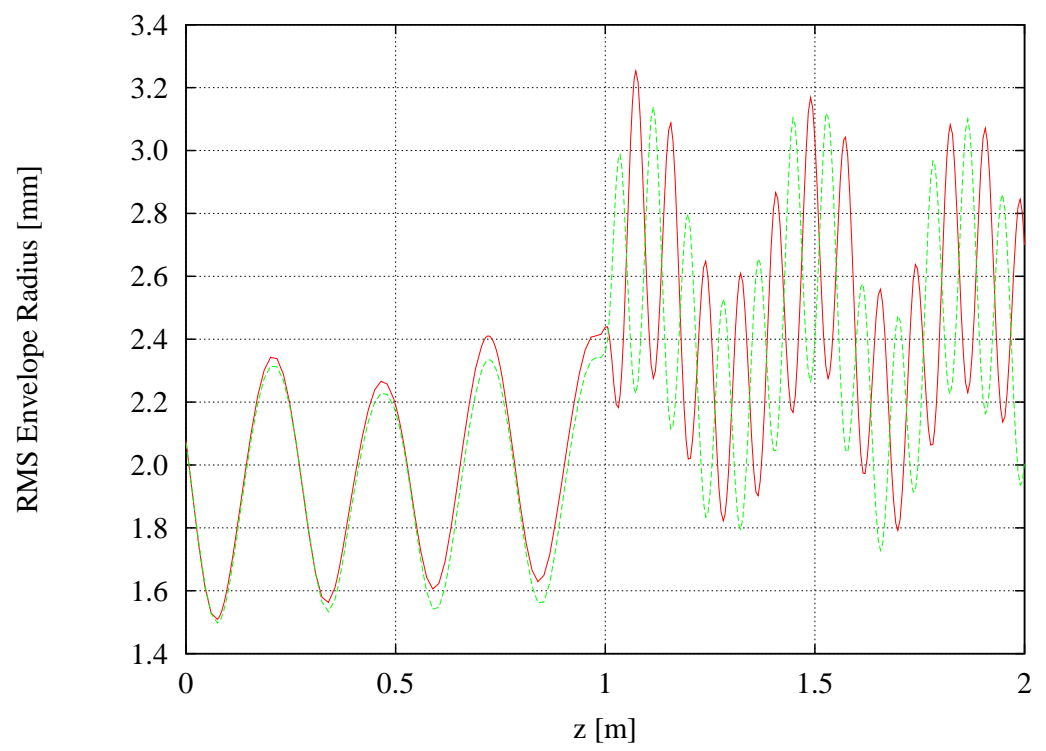

Figure 12.6: Beam transverse RMS envelope evolution in matching section.

distributions. These snapshots are taken at specific point along the beamline (see Figure 12.7) : (a) $z=0.98 \mathrm{~m}$; (b) $\mathrm{z}=1.0 \mathrm{~m}$; (c) $\mathrm{z}=1.01041667 \mathrm{~m}$; (d) $\mathrm{z}=1.0208333 \mathrm{~m}$; (e) $\mathrm{z}=1.03125 \mathrm{~m}$; (f) $\mathrm{z}=1.041667 \mathrm{~m}$; (g) $\mathrm{z}=1.0520833 \mathrm{~m}$; (h) $\mathrm{z}=1.07291667 \mathrm{~m}$; (i) $\mathrm{z}=1.09375$ $\mathrm{m}$; and $(\mathrm{j}) \mathrm{z}=1.1145833 \mathrm{~m}$. These plots are to compared with the envelopes of Figure 12.6. We see that the transverse envelope oscillations follow from betatron rotation in the transverse phase-space.
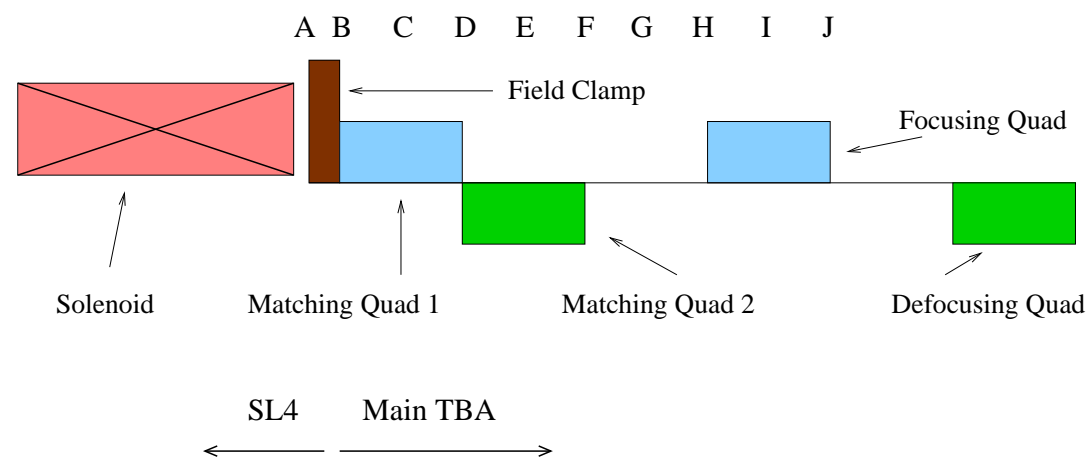

Figure 12.7: Observation points in the matching section. 
(a)

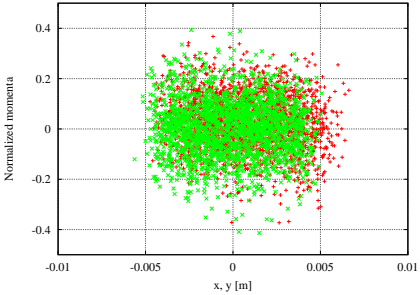

(c)

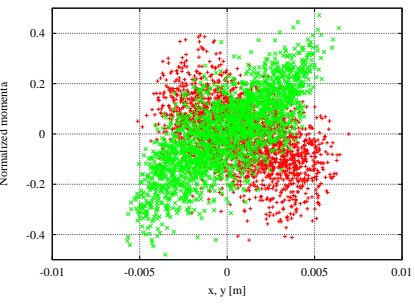

(e)

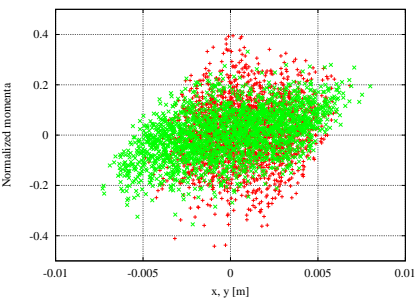

(g)

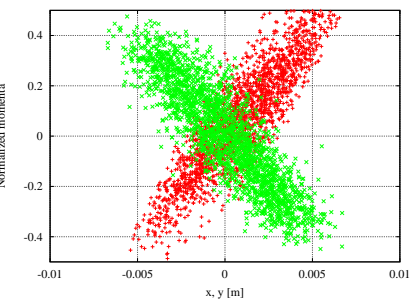

(f)

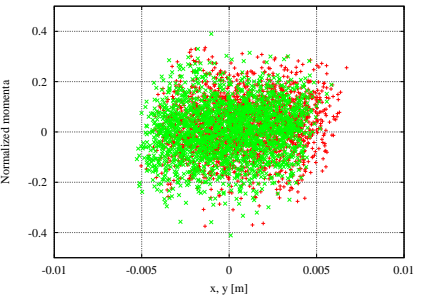

(d)

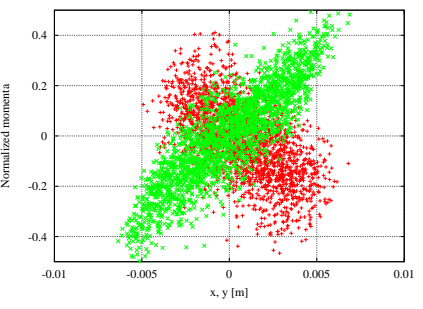

(h)
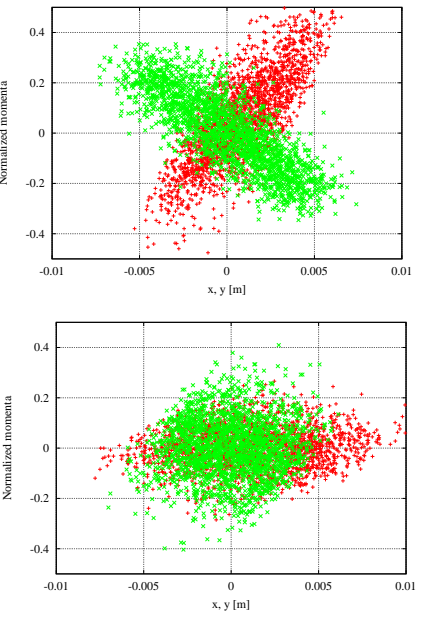

(i)
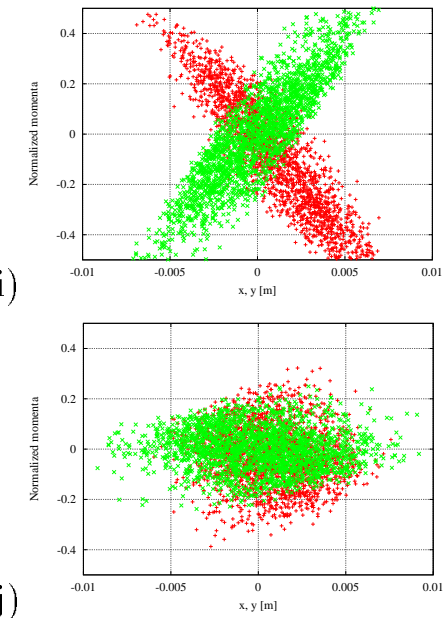

Figure 12.8: Transverse phase-space evolution in the matching section. 


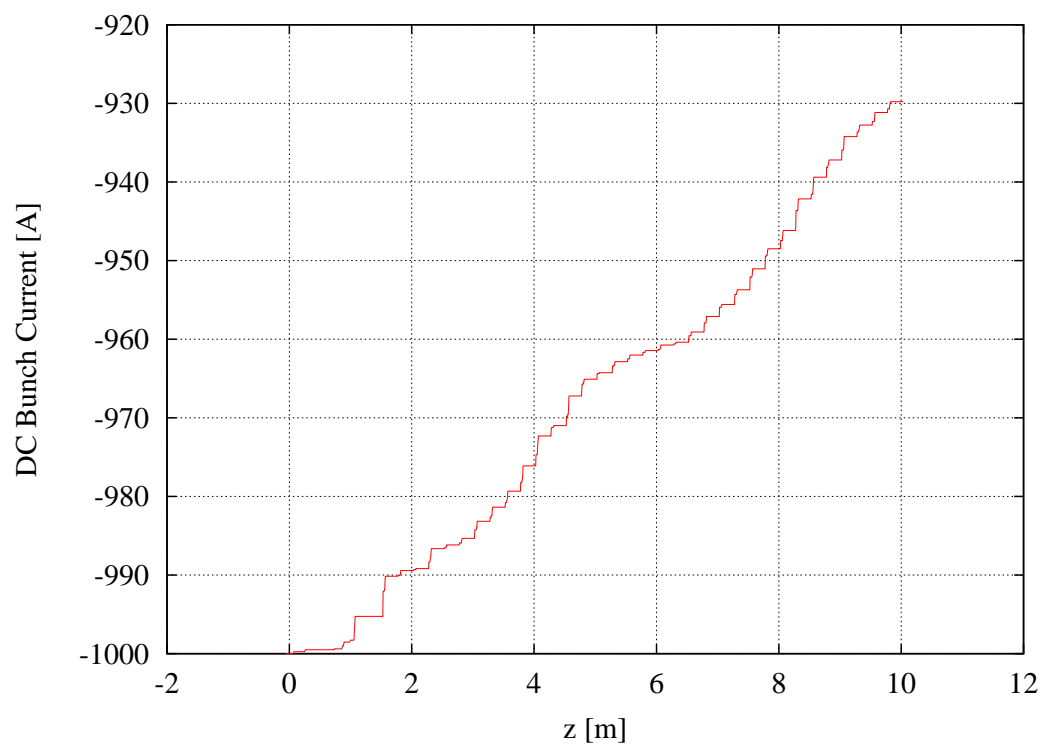

Figure 12.9: Bunch current evolution from matching section through main extraction section.

\subsection{Longitudinal Phase-Space Evolution}

The longitudinal dynamics of the rf bunches constitute the heart of any RK-TBA. The energy extracted from the bunches in the rf cavities must be replaced by the induction modules. The detuning of the cavity introduces a nonlinear correlation between energy and phase for particles within the bunch. This induces the bunch to rotate in longitudinal phase space. The re-acceleration in the induction modules is weakly dependent upon the dc beam current, but the rf cavity field excitation is strongly dependent upon both the dc current and the bunching parameter, $\mathrm{b}\left(\simeq \frac{1}{2} I_{r f} / I_{d c}\right)$.

In the simulation, we see that the DC current carried by a given bunch decreases at a slow rate over the length of the device (Figure 12.9), and the bunching parameter varies significantly along the beamline. In Figure 12.10, we show the variation of the bunching parameter along the beamline, with the positions of the rf output cavities indicated. This figure clearly shows the synchrotron oscillation that follows from the bunch rotation, shown in Figure 12.11. The longitudinal phase space distribution of the bunch is shown after passing through a cavity at various locations along the beamline.

We also see another effect of inductive detuning. The energy of a bunch in the steady-state portion of the beam pulse increases in the first segment of the main TBA beamline, as the bunching increases from its initial unbunched state to the final bunched 


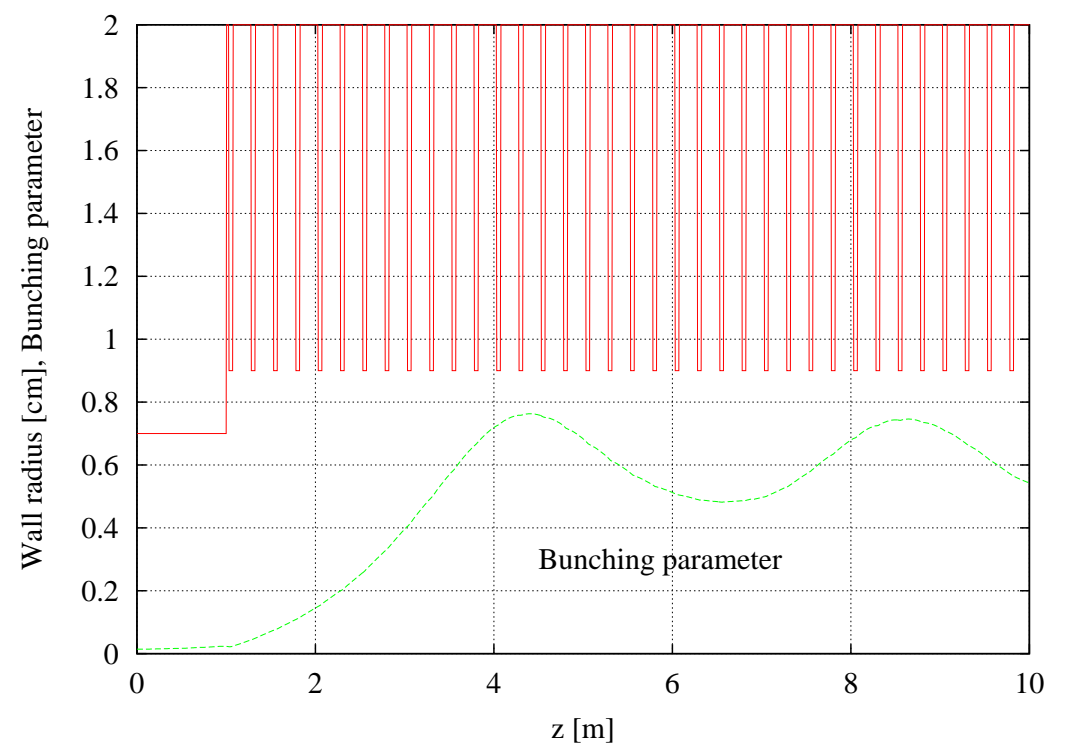

Figure 12.10: Bunching parameter variation along beamline.

(a)

I

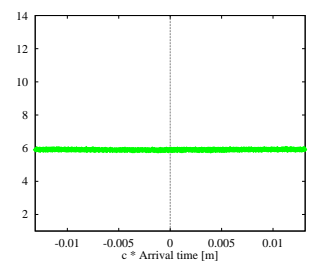

(d)

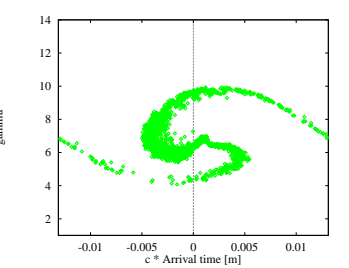

(g)

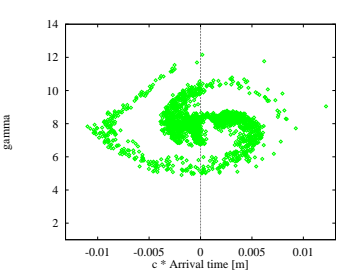

(b)

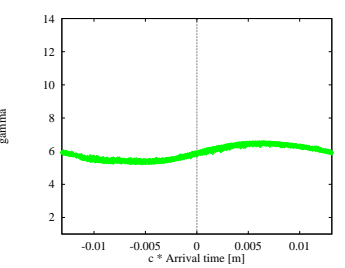

(e)

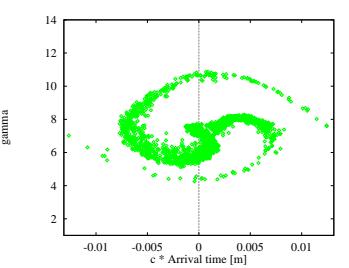

(h)

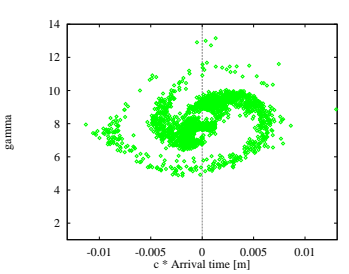

(c)

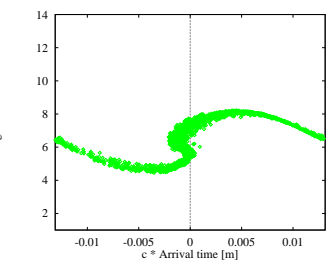

(f)

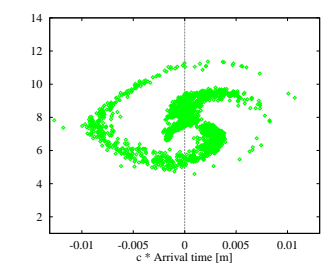

(i)

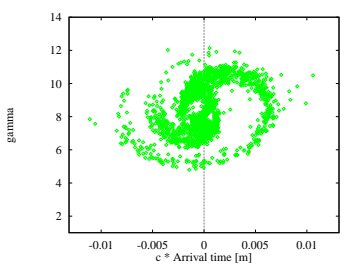

Figure 12.11: Longitudinal phase space distribution of the bunch after passing through cavities \#: 1 (a), 5 (b), 10 (c), 15 (d), 20 (e), 25 (f), 30 (g), 35 (h), and 40 (i). 


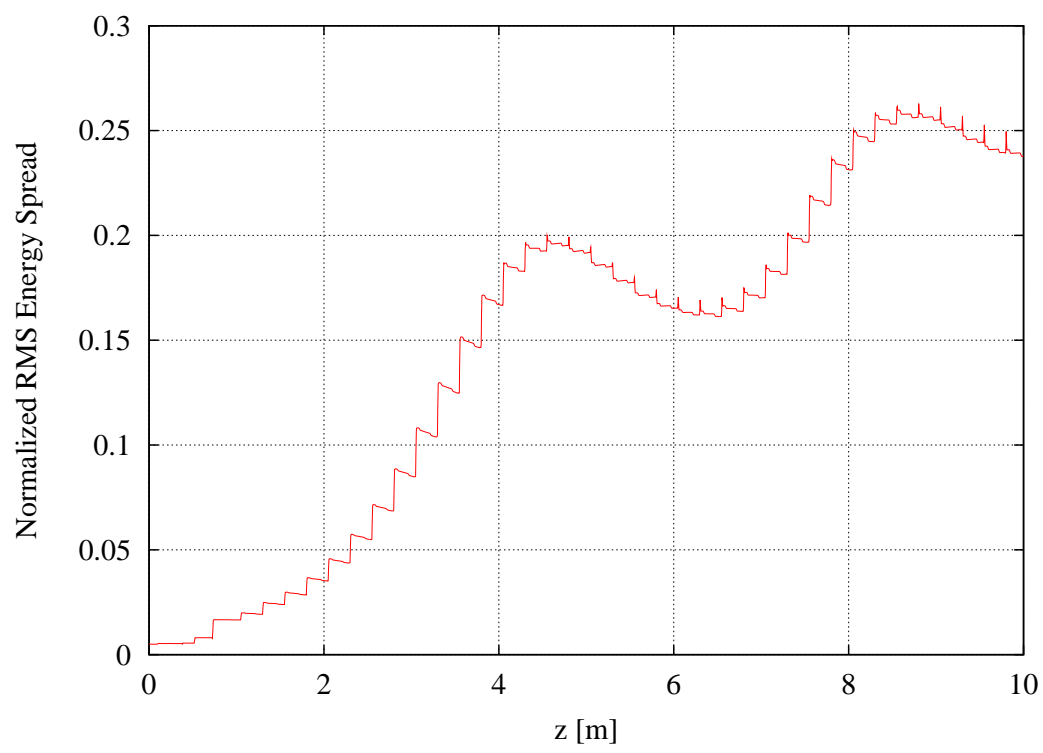

Figure 12.12: Evolution of normalized RMS energy spread.

state. Simultaneously, the energy spread of a bunch will increase from its inital to its final value (see Figure 12.12). The cavities are designed to extract their design power from bunches with the nominal design value of the bunching parameter (ie. $b=0.70$ ) and dc current. The beam will continue to gain energy from the induction modules until this equilibrium state is reached. Once the bunching has been achieved, there will be no net energy gain by the beam particles.

However, due to the synchrotron rotation required for longitudinal stability, the beam will experience periodic debunching and rebunching as it progresses along the beamline. Neglecting the small decrease in total current carried by an individual bunch, this will result in the bunch periodically losing more or less of its design energy as it passes through the series of cavities. This is shown in Figure 12.13. Here, we see that the net energy of the bunch gradually increases. This gradual increase in net beam energy can be eliminated by slightly decreasing the voltage in the re-acceleration gaps of the induction modules. However, the synchrotron rotation will still result in oscillation of the bunch energy about some equilibrium value. 


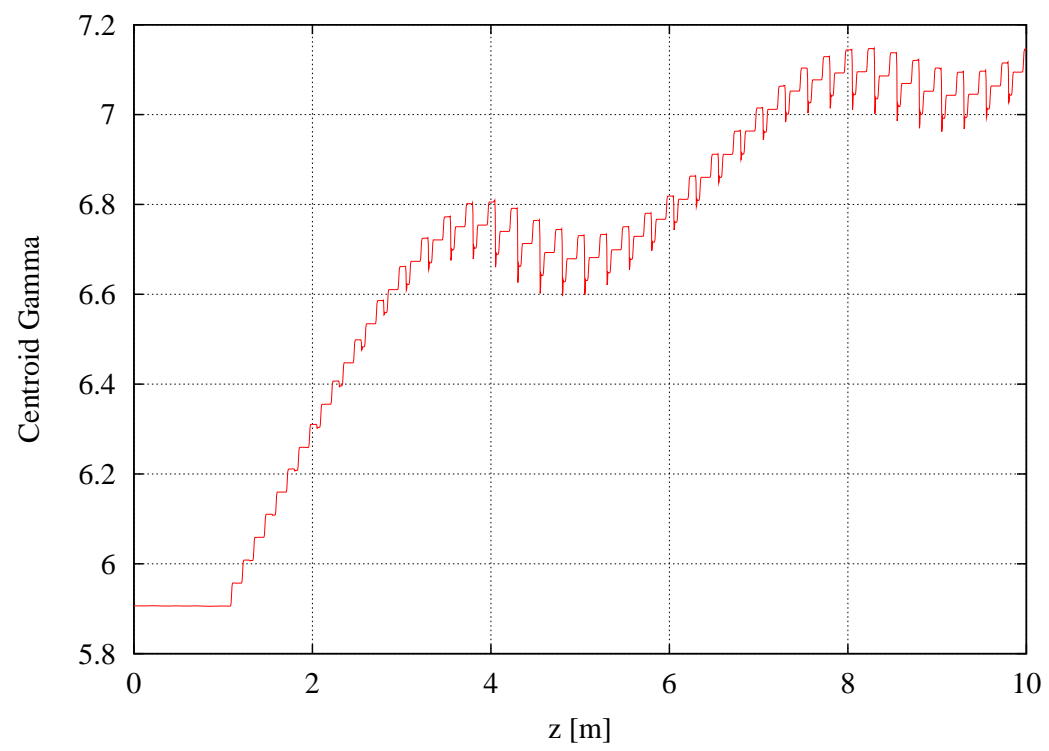

Figure 12.13: Variation of bunch centroid energy along beamline.

\subsection{Transverse Phase-Space Evolution}

The behavior of the bunches in longitudinal phase space effect the transverse dynamics as well. In particular we notice that an initially matched transport system, loses that feature as the bunch compresses and the bunch energy spread increases. The effect of bunch compression is to enhance the peak radial, defocusing self-fields in the presence of the conducting beampipe, while the enhanced energy spread introduces lower energy particles into the transport lattice. These two effects can, in principle, be dealt with by appropriately adjusting the strength of the quadrupoles along the beamline. In our studies we have maintained a constant parameter FODO lattice. In Figure 12.14 we show the evolution of the two transverse RMS envelopes along the beamline for bunches in the steady-state portion beam pulse. Visible is the slight increase in the average envelope radius at locations of peak bunching (near $\mathrm{z}=4 \mathrm{~m}$ and $\mathrm{z}=8.5 \mathrm{~m}$ ).

In Figure 12.15, we show the evolution of the normalized rms transverse emittance. The increase in emittance is only very slight. The first main degradation of the beam quality occurs in the matching section between the solenoid channel and the quadrupole lattice, as expected. However, by designing the two transport systems to have commensurate equilibrium transverse distributions, this increase has been kept small $(\sim 6 \%)$. After that, the beam quality experiences further slight degradation in the regions of the beamline where the beam is experience bunching or rebunching. Likewise, there is 


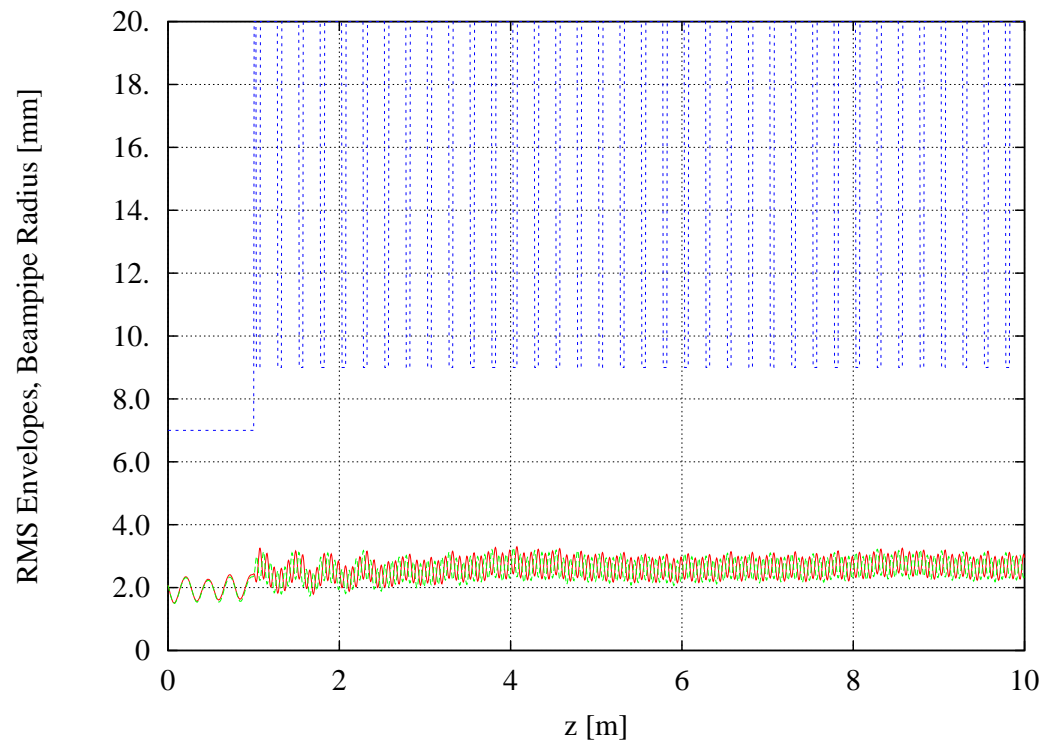

Figure 12.14: Evolution of transverse rms envelopes .

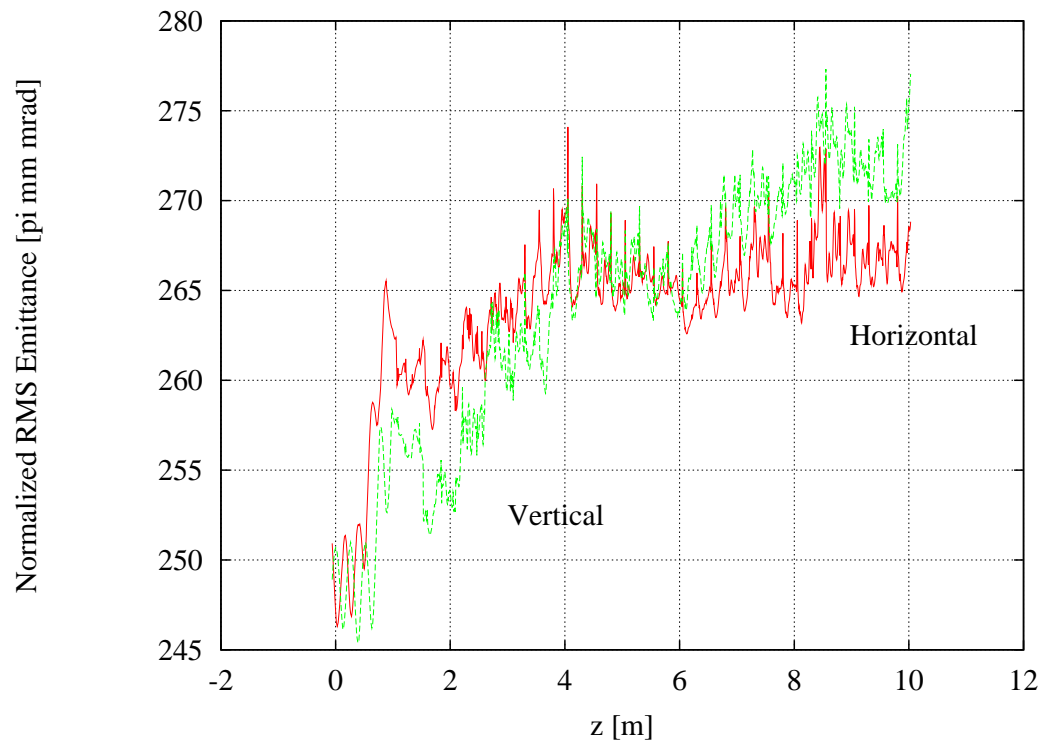

Figure 12.15: Evolution of transverse emittance. 
(a)

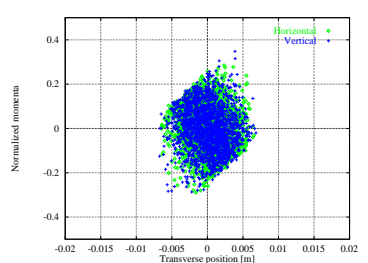

(d)

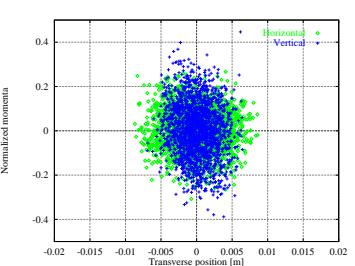

(g)

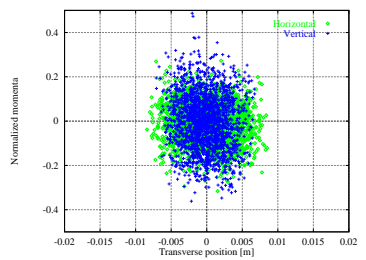

(b)

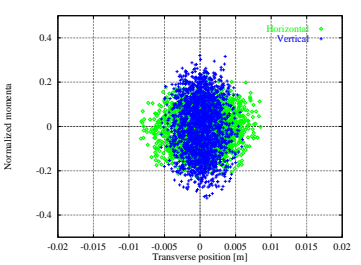

(e)

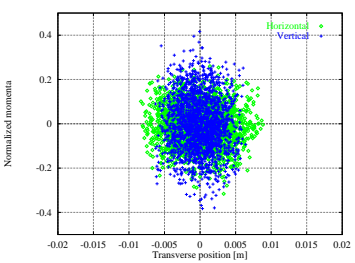

(h)

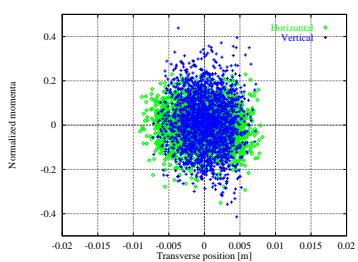

(c)

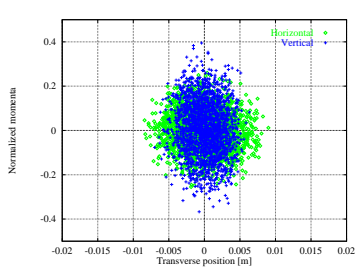

(f)

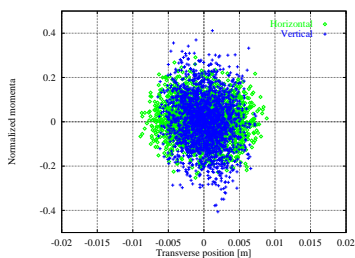

(i)

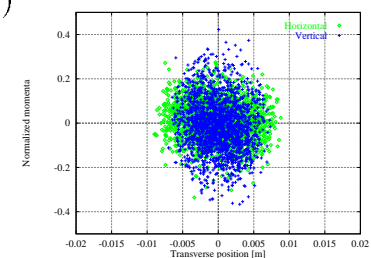

Figure 12.16: Transverse phase space distribution of the bunch after passing through cavities \#: 1 (a), 5 (b), 10 (c), 15 (d), 20 (e), 25 (f), 30 (g), 35 (h), and 40 (i).

a slight improvement as the beam undergoes the debunching cycle of the synchrotron oscillation.

The phase space distributions of the bunches at locations corresponding to the output planes of several cavities along the beamline is shown in Figure 12.16. It is seen that the distribution does not undergo any significant changes during propagation, and that the bunch remains stable along the beamline.

These simulations do not include the influence of transverse dipole modes in the induction modules or rf cavities. Thus, we have not included the important BBU instabilities that have shown to limit current transport in RK-TBA's. However, this simulation shows that pure rf monopole modes, 3-D space charge effects, and quadrupole transport do not significantly increase the transverse emittance in the beam.

\subsection{Dependence of RF Production Upon Beam Dynamics}

After an initial transient evolution of the cavity fields, the mode amplitudes enter the steady-state regime. This evolution is shown in Figure 12.17. We see that the output power levels in the main TBA section cavities vary by a factor of 2-3. The synchrotron 


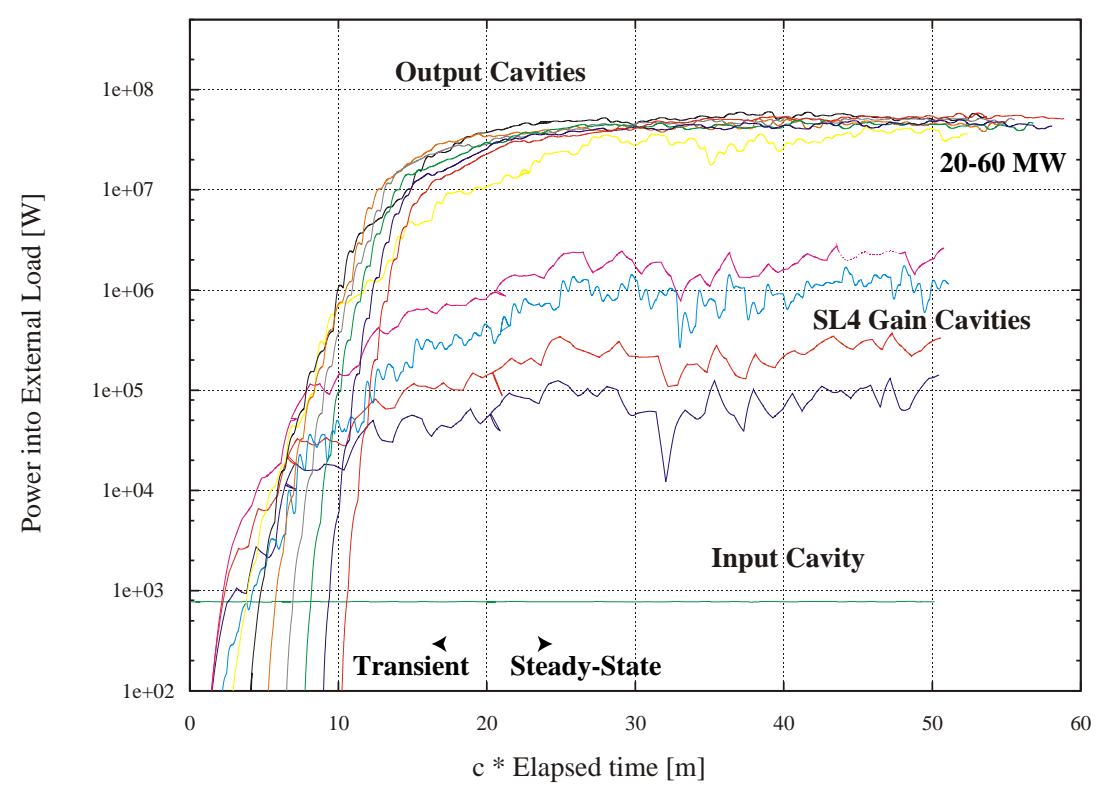

Figure 12.17: Transient to steady-state power output in the RTA.

oscillations affect the coupling of the bunch to the rf cavities, resulting in an oscillation along the beamline of the steady-state output power levels of the cavities, shown in Figure 12.18. Similarly, the output phases also show a periodic behavior. While remaining relatively constant in the regions of re-bunching and maximum compression, there is a rapid change in the phase during the cycles of de-bunching and minimum compression.

(a)

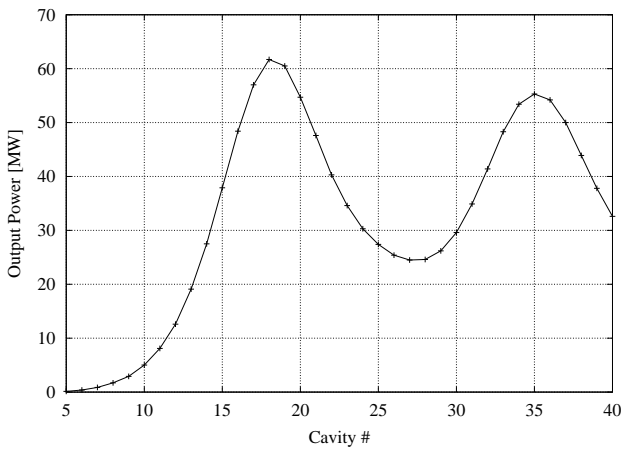

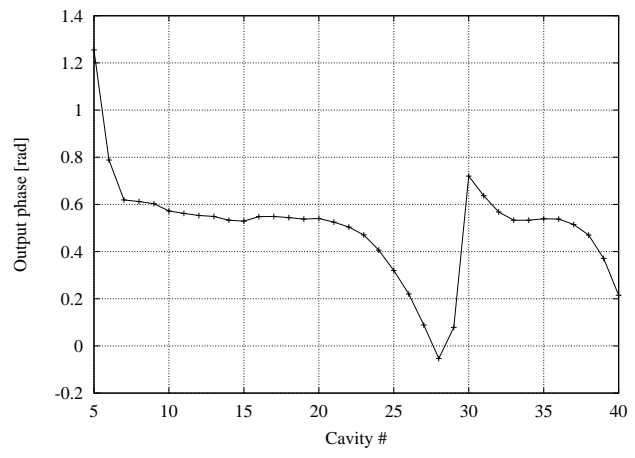

Figure 12.18: Steady-state output power (a) and phase (b) in the main TBA section. 
(a)

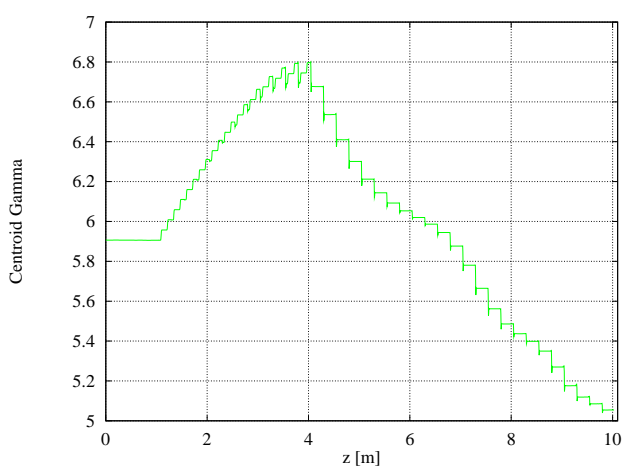

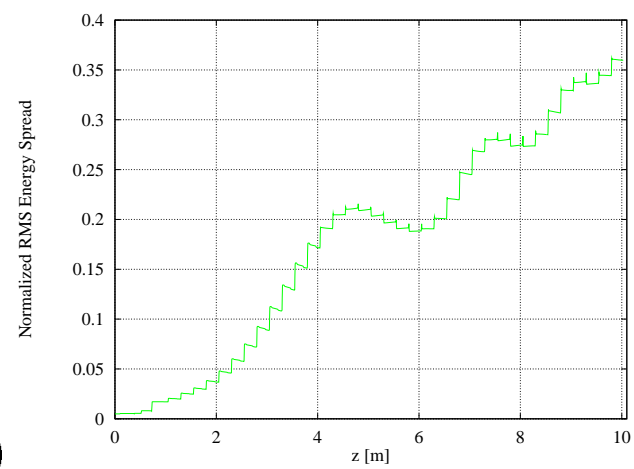

Figure 12.19: Variation of (a) bunch centroid energy and (b) normalized RMS energy spread.

\subsection{Extension to an Afterburner Configuration}

This beamline design also gives us the ability to study beam dynamics in the afterburner section of a main TBA. Below I present the results of simulations that detail the behavior of the beam in this same design, without the benefit of re-acceleration after $z=4 \mathrm{~m}$. To perform this study, I took the results and maps from the previous test case (once steadystate had been reached), then restarted the simulation with no voltage applied across any of the induction gaps located after $\mathrm{z}=4 \mathrm{~m}$. Then, steady-state equilibrium was again obtained.

The evolution of the average energy and energy spread of the bunch along the beamline is shown in Figure 12.19. We see that without the benefit of re-acceleration, the average energy in the bunch will decrease at a steady rate. As a result of this decrease in the bunch energy, the normalized energy spread will continue grow.

The transverse phase space is affected by this decrease in bunch energy. The transverse components of the Lorentz force are proportional to $1 / \gamma$, and hence become more important as the energy drops. We see the emittance growing rapidly as the bunch energy continues to drop, followed by the growth in the RMS beam envelopes (Figure 12.20). The transverse phase distribution of the bunch after the 40th rf cavity is shown in Figure 12.21. The vertical phase-space distribution has become significantly diluted, increasing from approximately $270 \pi \mathrm{mm}$-mrad in the previous scenario (see Figure 12.15) to approximately $310 \pi \mathrm{mm}$-mrad in the present case, with the majority of this growth occuring in the last 2 meters of beamline.

The increase in emittance and the beam envelope causes a slight increase in the rate of current loss. Meanwhile, the longitudinal Lorentz force is proportional to $1 / \gamma^{3}$ so 
(a)

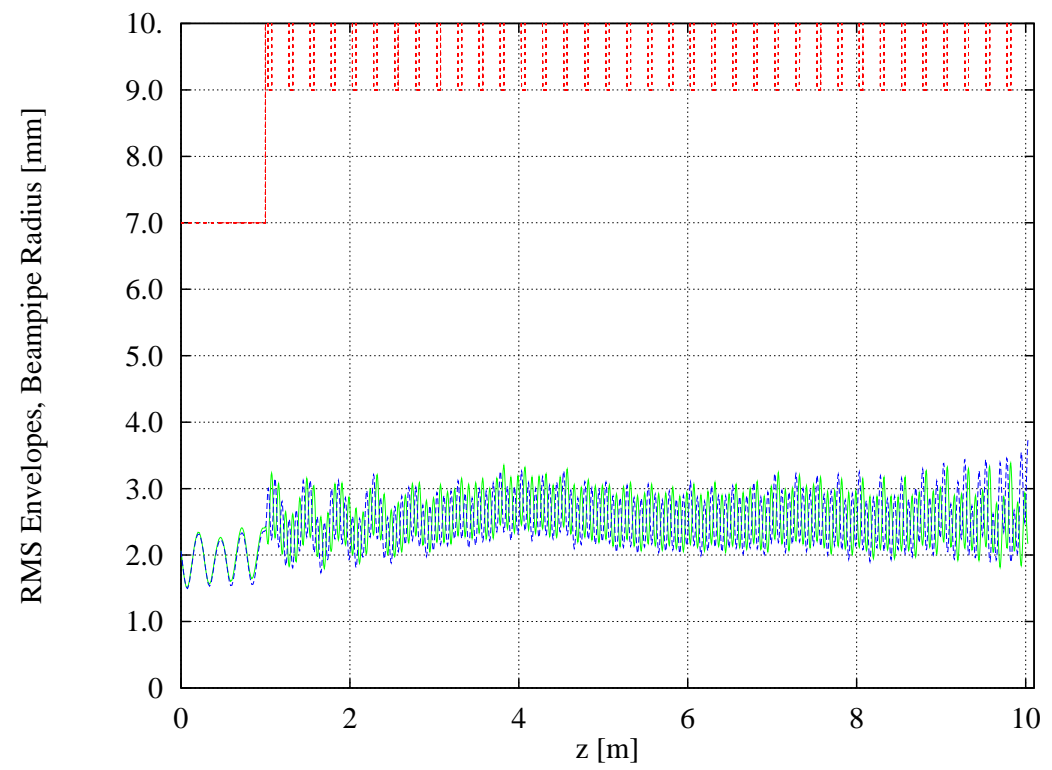

(b)

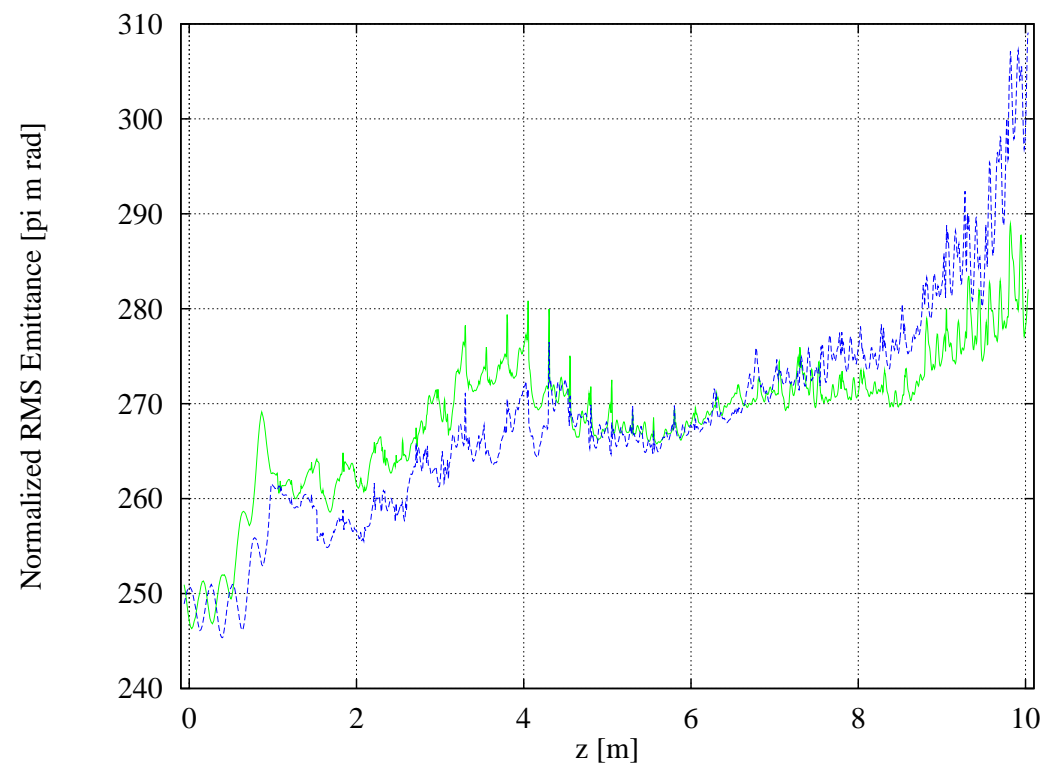

Figure 12.20: Transverse RMS envelopes (a) and emittance (b) evolution. 


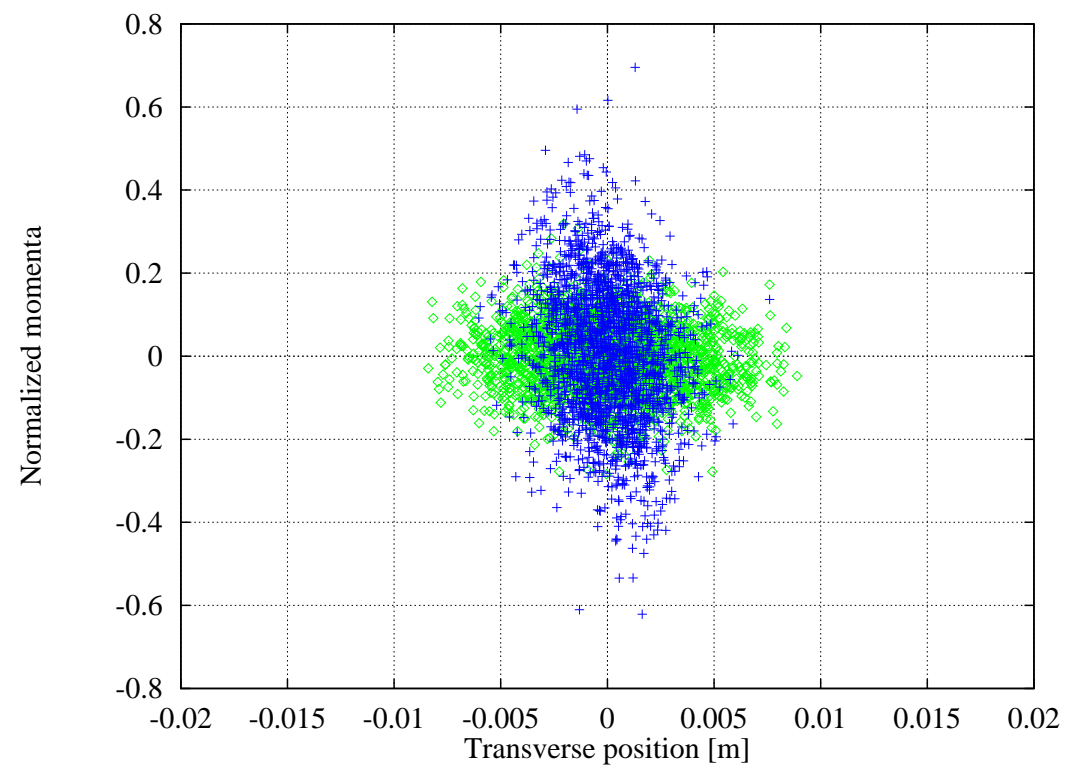

Figure 12.21: Transverse phase space distribution after 40 rf cavities.

that the drop in bunch energy and increase in energy spread results in a shortening of the synchrotron oscillation period (see Figure 12.22). This effect also shows up in the output power and phase variation in the cavities along the beamline (Figure 12.23). We also see that the beam quality in the longitudinal phase space is greatly diminished after the 40th cavity (Figure 12.24), compared to our previous scenario (Figure 12.11).

(a)

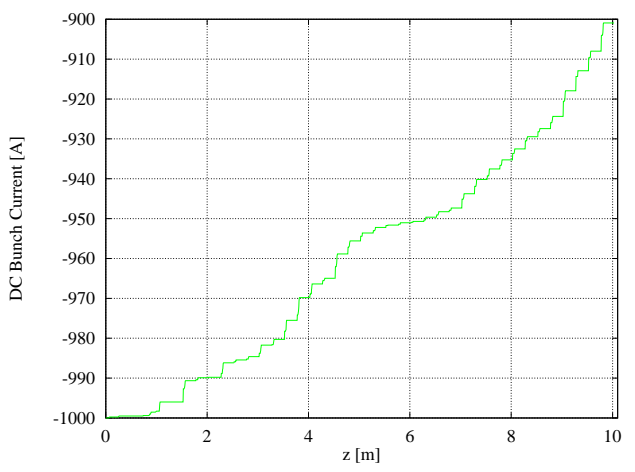

(b)

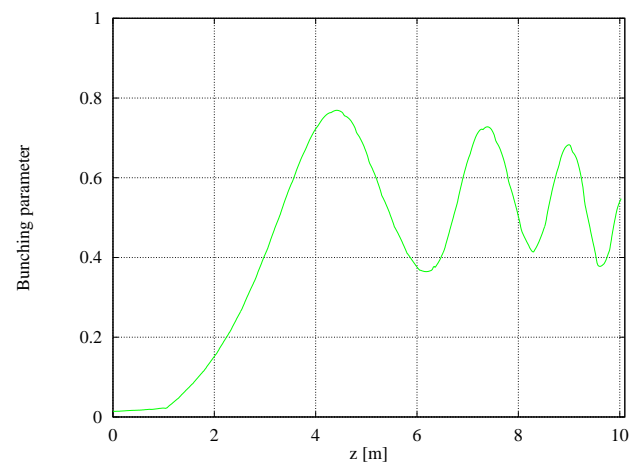

Figure 12.22: Current (a) and bunching parameter (b) evolution in the afterburner configuration. 
(a)

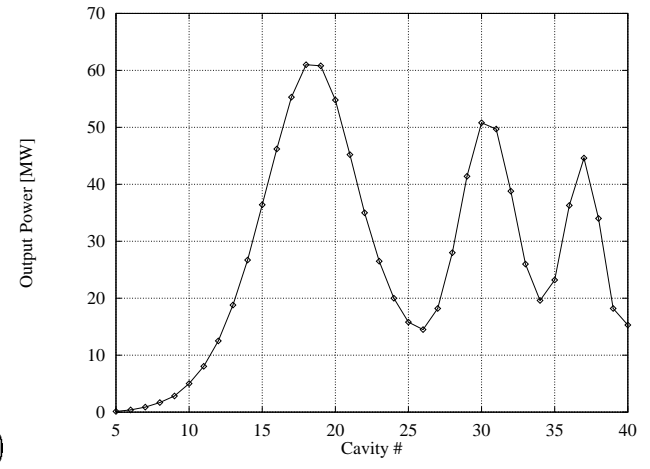

(b)

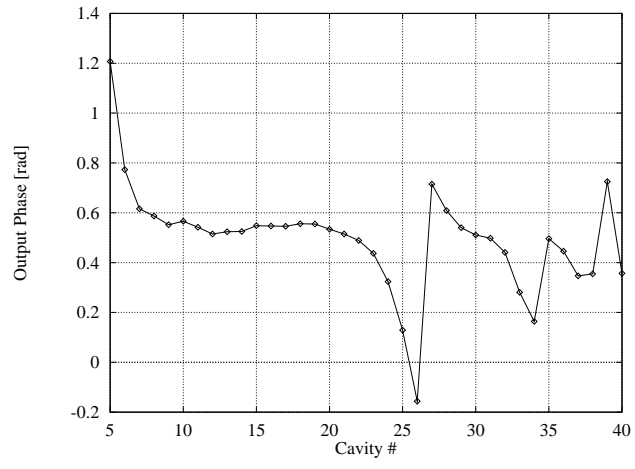

Figure 12.23: Steady-state output power (a) and phase (b) in the afterburner configuration.

(a)

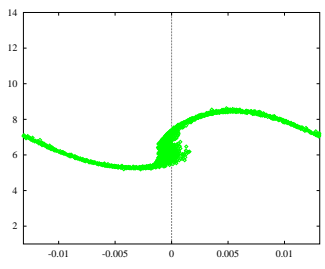

(d)

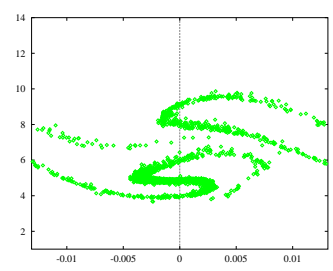

(b)

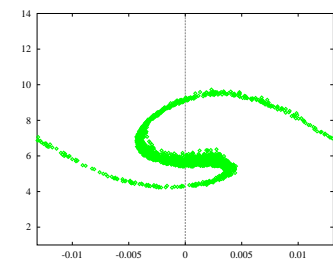

(e)

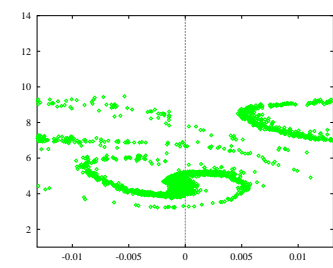

(c)

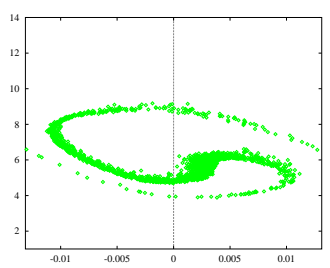

(f)

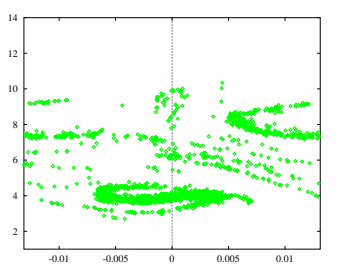

Figure 12.24: Longitudinal phase space ( $\gamma$ vs. $c^{*}$ Arrival time ) evolution, from cavities 15 (a), 20 (b), 25 (c), 30 (d), 35 (e), 40 (f). 


\subsection{Summary}

In this Chapter, the results of a detailed simulation of the beam dynamics in a proposed RTA beamline have been presented. We have seen that from a proper design of the beamline parameters, and, in particular, of the matching section, that the degradation of the beam quality (as quantitatively measured by the beam emittance) can be kept to quite tolerable levels. This design is guided by consideration of the equilibrium transverse distributions in the two different transport channels. The longitudinal sector of the beam dynamics proves to be the more difficult one to match. This will be true of any TBA device that attempts to match into a channel with constant rf cavity parameters. To avoid the large variation in output power levels from the various cavities along the beamline, it will be necessary to incorporate a second-order design strategy. This strategy may vary the parameters of the cavities to attempt to achieve nearly constant rf power extraction from every cavity. Another possible solution would be to use cavities with somewhat smaller detuning angles than the 1.2 radians employed here. In that case the initial rate of bunching would decrease due to the smaller head-tail energy spread, requiring more periods until equilibrium is reached. But, the resulting peak-to-trough variation in the bunching parameter over the course of a synchrotron oscillation will be comensurately smaller. 


\section{Appendix A}

\section{RTA Project}

A test facility, called the RTA, has been established at LBNL [95] to verify the analysis used in the TBNLC design study. The primary effort of the facility is the construction of a prototype RK-TBA subunit that will permit the study of technical issues, system efficiencies, and costing. The layout of the proposed experiment is shown in Figure 12.1. The experiment will include a high-current electron gun, a pre-acceleration section, a modulation and adiabatic capture section, a main TBA extraction section, and a beam dump and energy spectrometer. To date, the injector and associated pulsed power system has been constructed and is undergoing testing. Diagnostics have been installed to study beam energy and current, transverse distribution of the current density and emittance, and pulsed power behavior.

\section{A.1 RTA Injector}

A major part of our effort has been towards the design and construction of an electron source with much lower emittance than typical induction injectors. The electron source will be a $3.5^{\prime \prime}$-diameter, thermionic, flat-surface W-type cathode with a maximum shroud field stress of approximately $165 \mathrm{kV} / \mathrm{cm}$. An emission density of $20 \mathrm{~A} / \mathrm{cm}^{2}$ is required from the cathode to produce $1.2 \mathrm{kA}$ beam. The RTA injector, depicted in Figure A.1, has 72 induction cores, each driven at $14 \mathrm{kV}$. The voltage across the A-K gap is $1 \mathrm{MV}$. The cores are segmented radially to reduce the individual aspect $\Delta r / \Delta z$ ratio. The lower aspect ratio reduces the variation in core impedance during the voltage pulse simplifying the pulse forming network (PFN) design.

High-voltage tests (ie. no beam) to full operational parameters have been successfully completed on the injector. In operation, a $500 \mathrm{kV}$ potential is developed across the two 


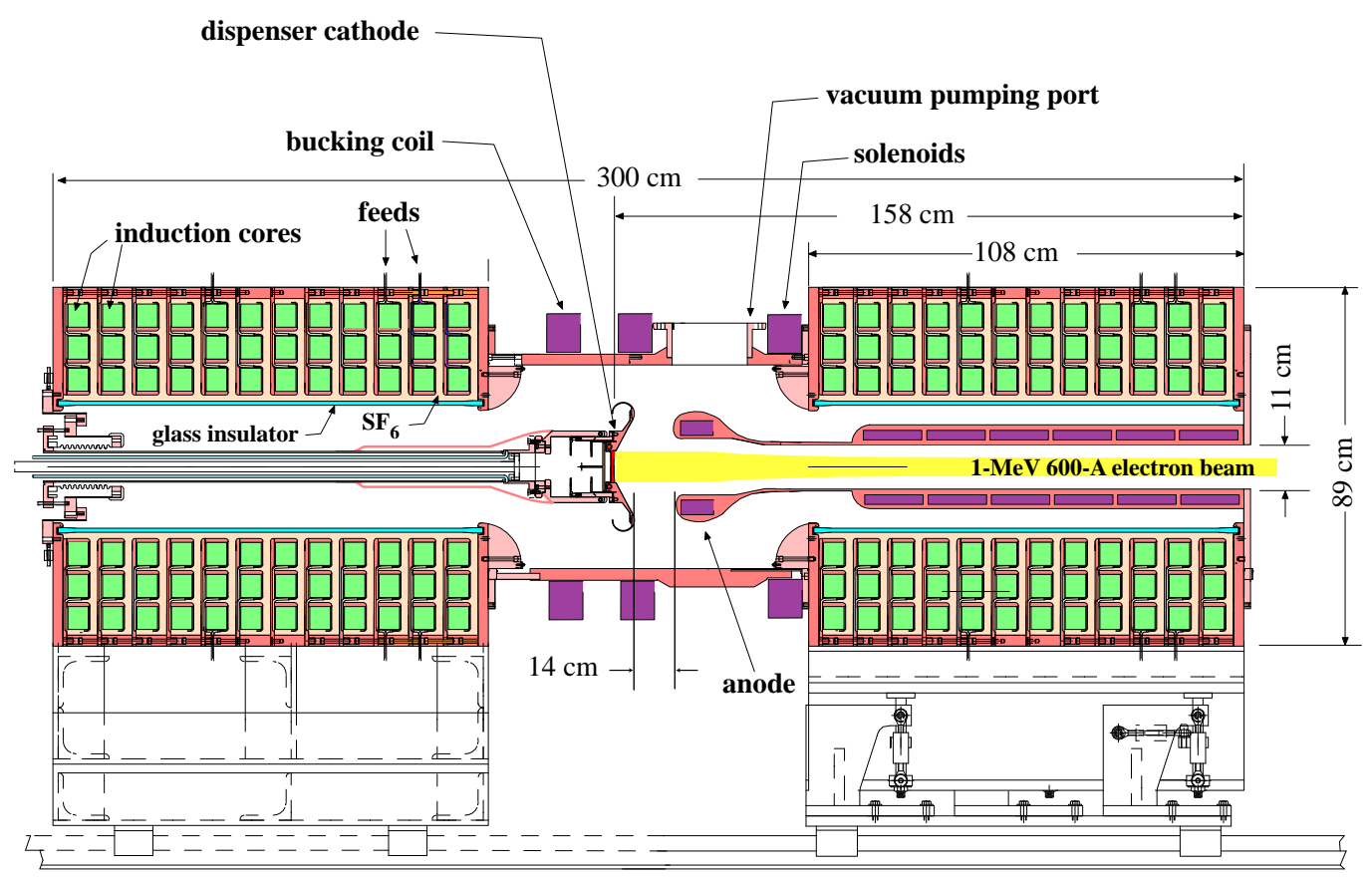

Figure A.1: Schematic of the RTA injector depicting the 24 3-core cells, electrodes, glass insulators, and solenoids.

30-cm-ID PYREX [96] insulators producing a $5.1 \mathrm{kV} / \mathrm{cm}$ average gradient along the insulator. The maximum field at the triple points, the intersection of insulator, vacuum, and metal, is designed to be less than $3.5 \mathrm{kV} / \mathrm{cm}$. Maximum surface field in the cathode half of the injector electrode is about $85 \mathrm{kV} / \mathrm{cm}$. The maximum field is about $116 \mathrm{kV} / \mathrm{cm}$ on the anode stalk.

Beam tests have also commenced. The A-K gap voltage was kept below $400 \mathrm{kV}$ producing almost $200 \mathrm{~A}$ beam current while radiation surveys, subsystem checkouts, and cathode studies were performed. No electrical breakdowns have been observed at these lower voltages. Vacuum can be maintained in the high $10^{-8}$ Torr range with a cold cathode increasing into the low $10^{-7}$ Torr range during operations. Theoretical perveance for the installed A-K gap width is $1.0 \mu$ pervs. Measured perveance is $\sim 0.84 \mu$ pervs with the uncertainty in the upper bound due to the present voltage calibration. The design voltage across the A-K gap in excess of $1 \mathrm{MV}$ has been achieved, and a maximum 780 A of beam current produced. This is consistent with the measured perveance.

Initial beam focusing in the injector is accomplished by three large-bore air-core solenoids installed on the central pumping spool. The first solenoid (the 'bucking coil') 
is operated to null the magnetic field from the other solenoids at the cathode front surface. There are seven smaller solenoids located within the anode stalk to provide additional focusing to transport the beam to the end of the injector.

\section{A.2 Pulsed Power System}

The injector's pulsed power system consists of a 20-kV High-Voltage Power Supply, 6-kJ Energy Storage Bank, two Command Resonant Charging (CRC) Chassis, 24 Switched Pulse Forming Networks, and four Induction Core Reset Pulsers. Each PFN drives a single 3 -core induction cell of the injector.

Segmenting the core in the induction cell and driving the individual core segments avoids a high-voltage step-up transformer. This reduces the developmental effort needed to achieve a "good" flattop pulse (minimal energy variation) with fast risetime and improves the efficiency of the overall pulsed power system. Our system of low-voltage PFNs driving multiple core induction cells is similar to the system envisioned for the extraction section in the full-scale RK-TBA design. For the injector core material, we choose 20- $\mu$ m-thick 2605SC METGLAS [97]. For the RTA extraction section we will use a lower loss 2714AS METGLAS for the induction cores.

Design of the switched PFNs follows easily from published METGLAS core loss data [98]. A flux swing of $2.6 \mathrm{~T}$ in $400 \mathrm{~ns}$ (FWHM) results in a magnetization rate of 6.5 $\mathrm{T} / \mu \mathrm{s}$ for the injector cores. This rate generates a loss density of $1800 \mathrm{~J} / \mathrm{m}^{3}$, or a $30 \mathrm{~J}$ lost in a 3 -core cell with $16.7 \times 10^{3} \mathrm{~cm}^{3}$ of $2605 \mathrm{SC}$ METGLAS. For an input voltage of $14 \mathrm{kV}$ applied for $400 \mathrm{~ns}$, these losses require that $5900 \mathrm{~A}$ be supplied to the cell. An additional $3600 \mathrm{~A}$ is required to supply beam current (1200 A x 3 cores/cell), resulting in a total current of $9 \mathrm{kA}$. The required drive impedance for a cell is then $1.5 \Omega$.

Achieving the fast risetime necessary to minimise the volt-seconds required for the injector cores presented a challenge. Budget constraints coupled with the large availability of EEV CX1538 thyratrons from the ATA program at LLNL made these tubes an attractive option. However, their poor time rate of current change (4 kA/ $\mu \mathrm{s}$ rating) made them questionable for this application, which requires about $40 \mathrm{kA} / \mu \mathrm{s}$. A variety of techniques were tried to decrease the risetime. Each thyratron is located between two current sheets connecting the PFN output to the output cables to reduce the stray circuit inductance. In addition, the thyratron ionization time is substantially reduced by applying a 1-2 A pre-pulse to the keep-alive grid 300-400 ns prior to the arrival of the main control-grid pulse. We presently have risetimes less than $150 \mathrm{~ns}$ for all 24 cells. 
Since the thyratron turn-on time is voltage dependent, we are adjusting the trigger time of each individual tube. Faster risetimes were achieved with Triton F-130 ceramic thyratrons. An upgrade of the current thyratrons in the injector pulsed power system should allow us to achieve the design 100-ns risetime.

At the $1-\mathrm{MV}, 1.2-\mathrm{kA}$ operating conditions we hope to produce a $\pm 1 \%$ injector voltage flat waveform for $120 \mathrm{~ns}$. We will need to adjust the number of turns in appropriate sections of individual PFNs to achieve this goal. Insertion of ferrite material in the center of the inductors coils will allow additional small corrections to the waveform. There are 24 EEV CX1538 thyratrons used in the pulsed power system for the injector.

\section{A.3 Injector Diagnostics}

A variety of diagnostics will be used to determine the performance of the injector, both permanently installed monitors for general operations and temporary diagnostics specific to injector commissioning and troubleshooting. Some of these are shown in the schematic for one of the diagnostic beamline configurations downstream from the injector (Figure A.2.)

\section{A.3.1 Current Measurements}

An accurate measurement of the emitted current from the cathode is required both for determining the performance of the injector and benchmarking codes. We have electrically isolated the cathode from the stalk, forcing the current to flow through several parallel, 0.25 inch wide, strips of $25-\mu \mathrm{m}$ thick stainless steel foil that act as current-viewing resistors (CVRs). The potential drop across the foil is measured and the current inferred. To improve the time response a parallel-strip shunt geometry is used where the foil is folded on itself to increase the resistance while lowering the series inductance. The beam dump is also electrically isolated similar to the cathode to allow the measurement of the total current deposited in the dump. Four CVRs, equally spaced azimuthally around the dump, are summed. A resistive wall current monitor ('beam bug', or 'BB') is located immediately following the injector. This monitor measures

the potential drop of the return wall current across a known resistance generating a signal proportional to the current. The potential drop is measured at eight locations around the circumference of the beam tube permitting the measurement of total current and centroid position. Magnetic pickup ('B-dot') loops are available to be inserted in the transport section after the injector to determine the time derivative of the current 


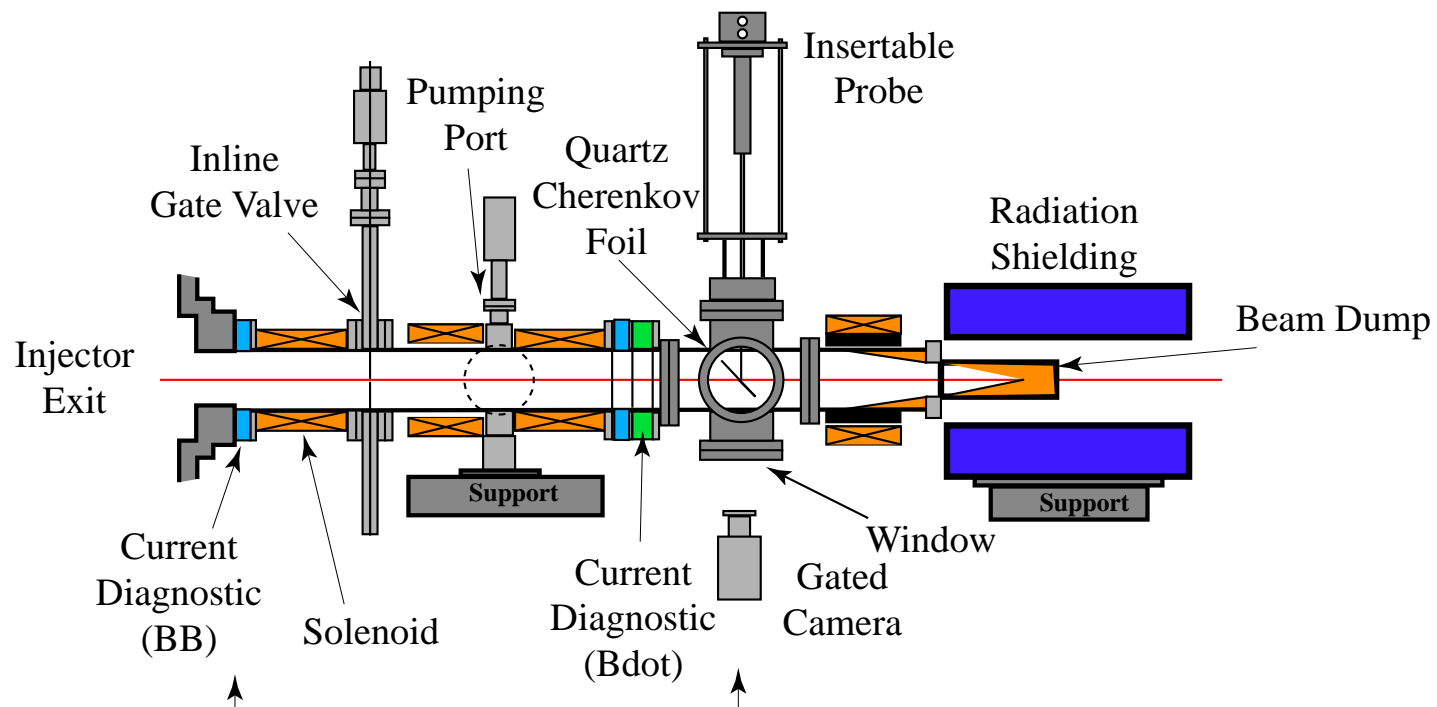

\author{
1.7 meters \\ from cathode \\ 2.8 meters \\ from cathode
}

Figure A.2: RTA Injector downstream. 


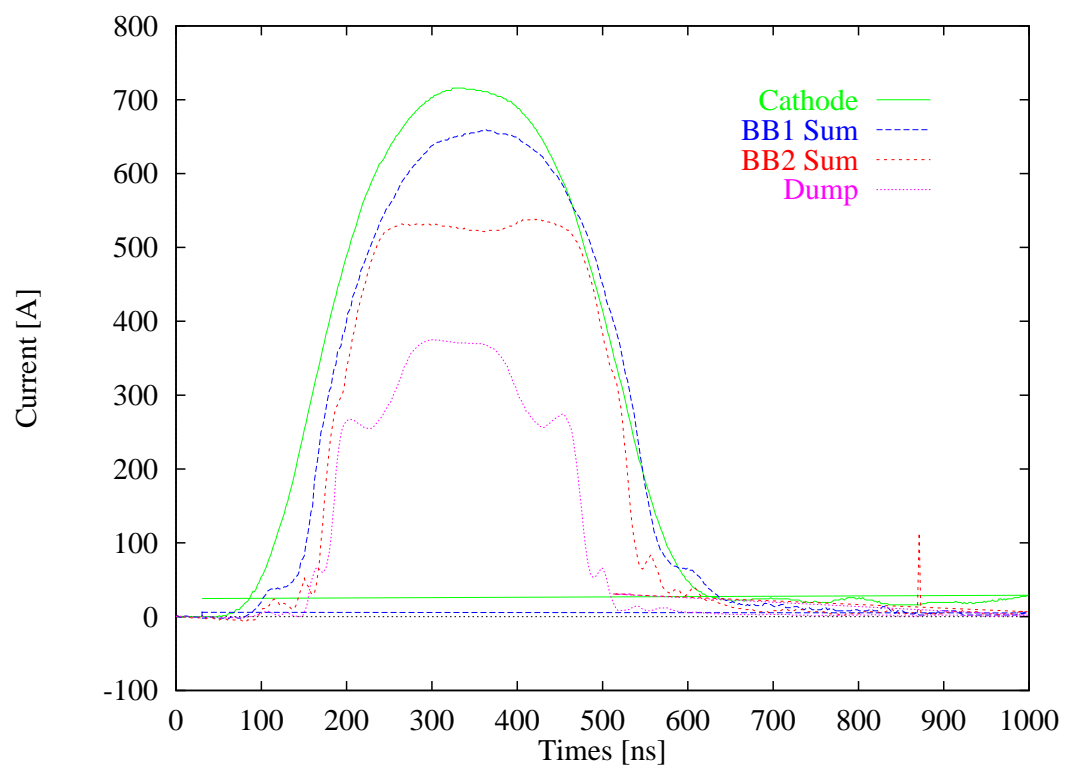

Figure A.3: Current measurements along the RTA beamline.

pulse. The voltage induced on the loop can then be integrated to recover the current and centroid position. The advantage of the B-dot loops over the other current diagnostics is a higher frequency bandwidth. The current profiles at various stages along the beamline are shown in Figure A.3.

\section{A.3.2 A-K Voltage and Beam Energy}

We are using two methods to determine the $\mathrm{A}-\mathrm{K}$ voltage and infer the beam energy. The first method involves using resistive voltage dividers (VRD) to measure the applied voltage to the induction cores at the power feed connections. Capacitive $\mathrm{dV} / \mathrm{dt}$ pickup probes [99] are used for a more direct measurement of the A-K gap voltage and also to provide greater bandwidth with respect to the resistive dividers. We also hope to employ a conventional energy spectrometer comprised of an on-axis collimator, dipole magnet, scintillator, and viewing port to directly measure the beam energy.

The injector has 72 cores organized into 24 sets of cells. We monitor the injector voltage by observing the voltage sums of 8 cells together. There are 3 of these sums that each represent one-third of the total voltage across the A-K gap. These sums and the total voltage waveform are shown in Figure A.4. 


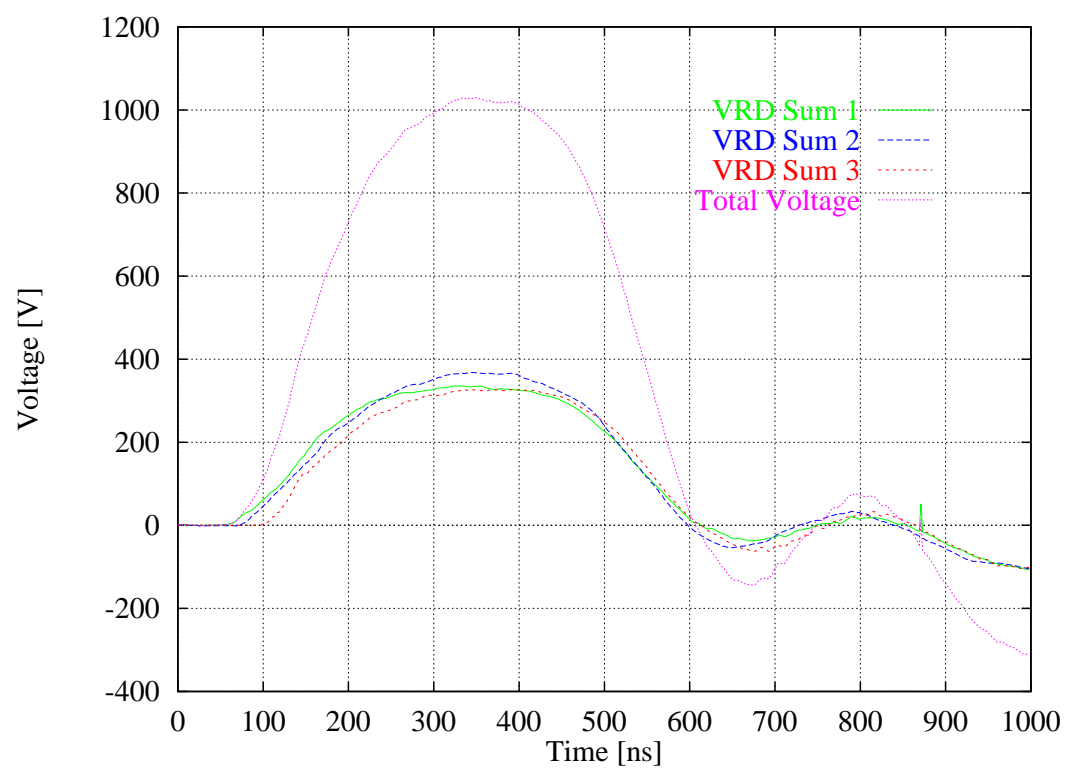

Figure A.4: Injector voltage diagnostic measurements.

\section{A.3.3 Current Density Profile}

The current density profile will be measured using Cherenkov radiation from intercepting foils (see Figure A.5). A primary concern with using foils is possible damage from beam energy deposition. Adjusting the repetition rate of the injector can control average heating of the foil. The difficulty is single shot heating where material can be melted and ejected before the heat is conducted away. To avoid damage to a thin quartz foil, we anticipate that the beam diameter must be greater than $2 \mathrm{~cm}$ for a $1-\mathrm{kA}, 300-$ ns, relativistic electron beam. The light generated at the beam/foil interaction will be recorded using both gated and streak cameras. The streak camera will be used principally to determine if the properties of the foil and/or beam change during the pulse. The significant levels of energy deposited in the foil could affect the dielectric constant or generate a surface plasma that could be confused as a variation in beam parameters.

\section{A.3.4 Emittance Measurement}

Measuring the beam emittance is expected to be very difficult as the beam is highly space charged dominated. A pepperpot emittance dianostic has been constructed. The size of the apertures is the only variable for adjusting the relative contribution of emittance to 


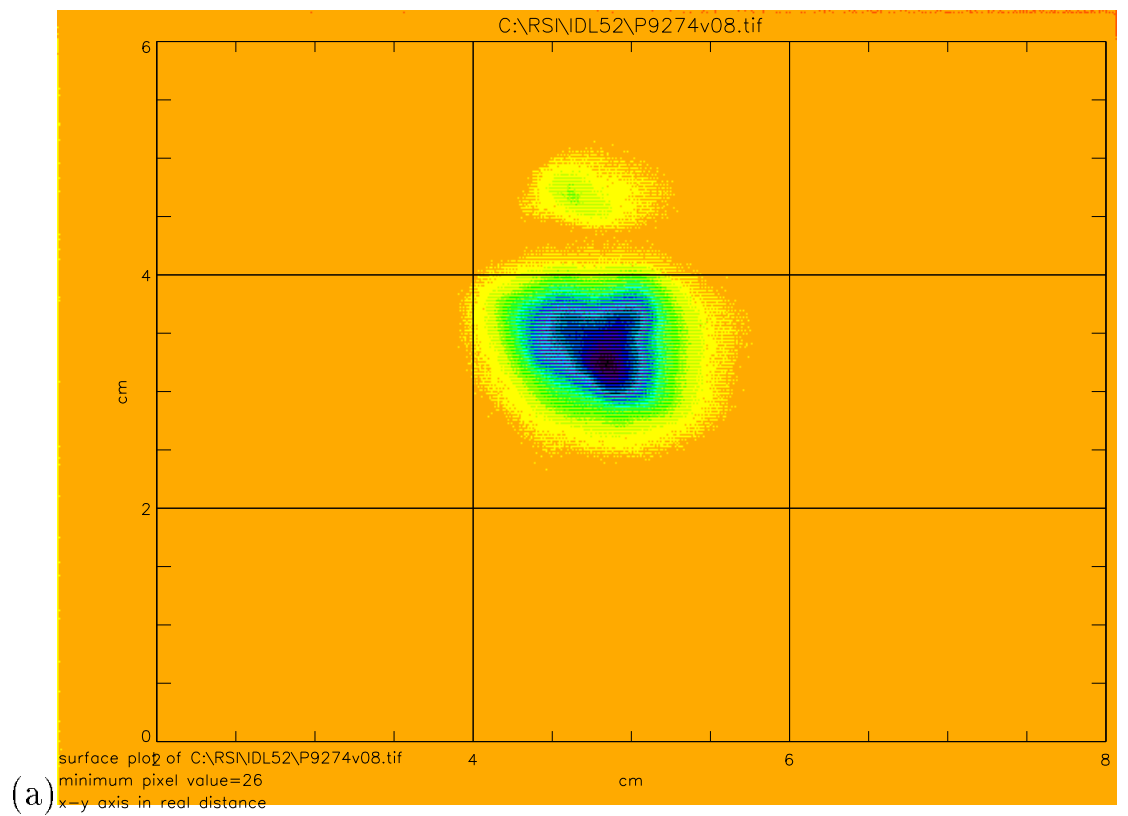

(a) axis in real distance $y_{\text {_ave }}=3.95093 \mathrm{~cm}$ $x_{-s d}=0.995346 \mathrm{~cm}$ $\mathrm{y}_{\text {sd }} \mathrm{sd}=0.771148 \mathrm{~cm}$

(b)

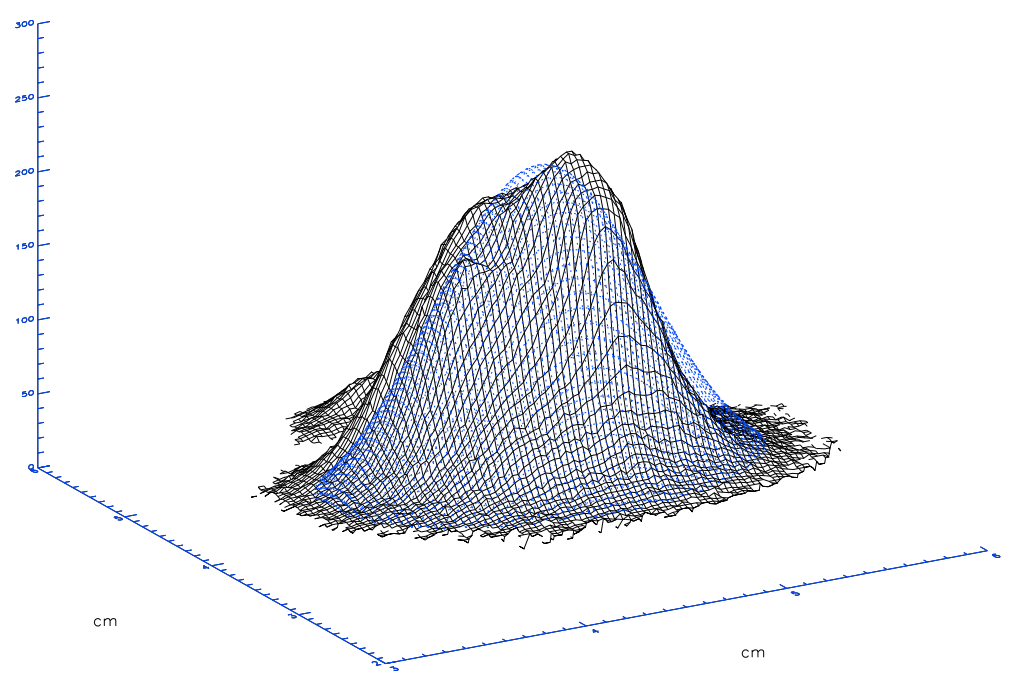

Figure A.5: Transverse beam profile: (a) false color intensity map; and (b) intensity surface plot and gaussian best-fit. 
space charge. For the designed RTA injector beam parameters, $1.2 \mathrm{kA}, 1 \mathrm{MeV}$ and 100 $\pi$-mm-mr, and using a $250-\mu \mathrm{m}$ aperture, the emittance term for the beamlets passing through the aperture plate is approximately an order of magnitude larger than the space charge term. Our aperture plate will consist of a rectangular pattern of 121 (11x11) 250$\mu \mathrm{m}$ apertures with $7 \mathrm{~mm}$ spacing on a $500-\mu \mathrm{m}$ thick tungsten plate. The tungsten plate represents about two range thickness for $1-\mathrm{MeV}$ electrons. The beamlets will strike a phosphor coated foil located about $80 \mathrm{~cm}$ after the aperture and imaged with a gated camera. 


\section{Appendix B}

\section{Experimental Studies in Ka-Band and Code Validation}

\section{B.1 Experiments with PIVAIR at $6.7 \mathrm{MeV}$}

An experiment to produce a $35 \mathrm{GHz}$ modulated beam by a FEL, and to subsequently extract power from this beam in a resonant cavity was performed at the CESTA facility of the CEA in Le Barp, France during January-February, 1999. This work is in support of the study of two beam accelerators based on relativistic klystrons, and has been executed jointly by groups at LBNL, CEA/CESTA, and CERN. One of the aims of this current experiment is to validate the RK-TBA principle at higher frequencies, and to benchmark our simulation codes.

For the design of the experiment and the analysis of its results, we have relied upon several distinct codes: an envelope code for basic transport studies; PARMELA [100] for

more detailed examination of the transverse phase space evolution; SOLITUDE [101] for the study of 3D, steady-state FEL interactions; RKS (Chapter 11 ) to study the timedependent, 3D bunch evolution and power production in output microwave cavities; and GdifdL [102] for the detailed study of the electromagnetic modes in the cavities.

\section{B.1.1 PIVAIR Accelerator}

A drawing of the accelerator PIVAIR is shown in Figure B.1. The high voltage pulse generator of the injector was developed by Pulse Science Incorporated (PSI) for the Dual Axis Radiographic Hydro-Test Facility (DARHT) and the diode was designed by Los Alamos National Laboratory (LANL). It is a single shot device whose repetition rate 


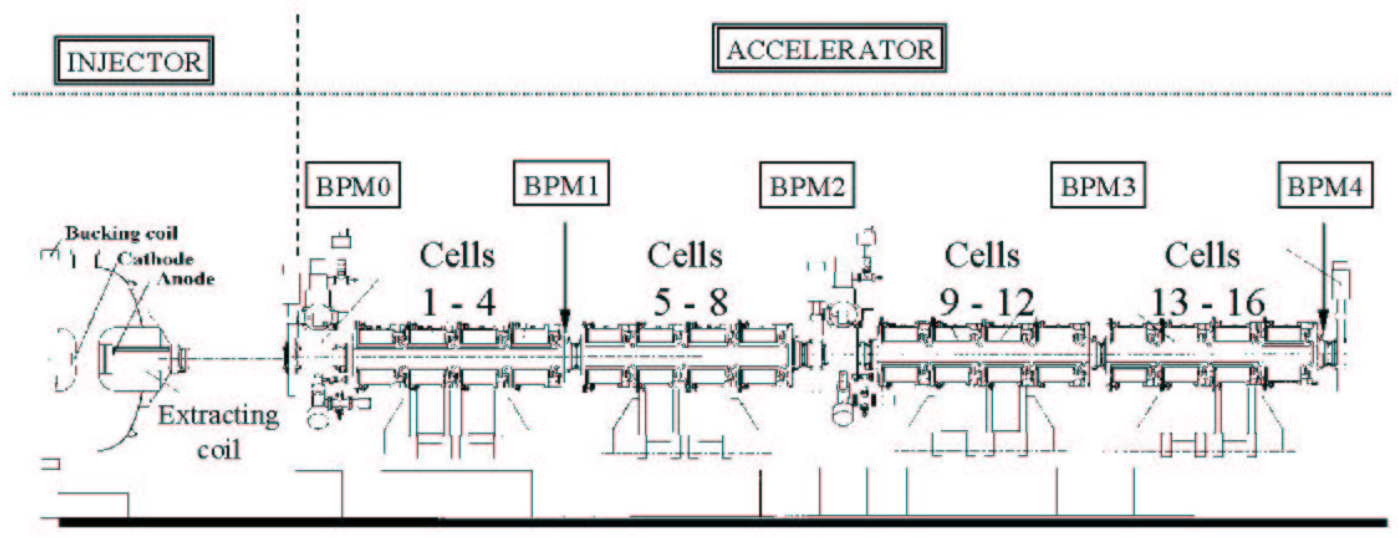

Figure B.1: PIVAIR Injector and accelerator.

is once every 5 minutes. A $3 \mathrm{kA}, 3.5 \mathrm{MeV}$ electron beam is extracted from a velvet cathode with excellent reproducibility. Upon leaving the injector, whose exit is shown at the left of Figure B.1, the electron beam is accelerated to $6.9 \mathrm{MeV}$ by 16 induction cells with good reliability. Each includes 11 ferrite cores, a solenoid magnet for beam guiding, 2 trim coils for beam steering and a $19 \mathrm{~mm}$-long accelerating gap. The induction cells are fed by external high voltage generators producing $210 \mathrm{kV}, 75$ ns pulses. Each generator drives two cells. Five beam position monitors (BPM) are installed between each block of four cells and both ends. The beam energy spread is less than $1 \%$ over 60 ns as determined by a time resolved spectrometer measurement and the normalized edge emittance is $1000 \pi-\mathrm{mm}$-mrad.

\section{B.1.2 Experimental Set-Up}

In Figure B.2 the experimental layout for the cavity experiment is shown, with PIVAIR indicated schematically at left. On the right are shown two alternative ends for the beam line, the upper for measuring the cavity output power, and the lower for beam position and size (with an inclined thin mylar target), and bunching measurements (with a thick fused silica target) using optical diagnostics which have been described elsewhere [103]. The full current from PIVAIR was collimated to $830 \pm 30 \mathrm{~A}$ at the FEL entrance. The FEL properties are listed in Table B.1. The input electromagnetic signal of $5 \mathrm{~kW}$ at $35 \mathrm{GHz}$, generated by a magnetron, is then amplified in the wiggler by the electron beam. In order to bring the electron beam onto the ideal helical trajectory, an adiabatic section six periods long is used to increase the wiggler magnetic field from zero to its 


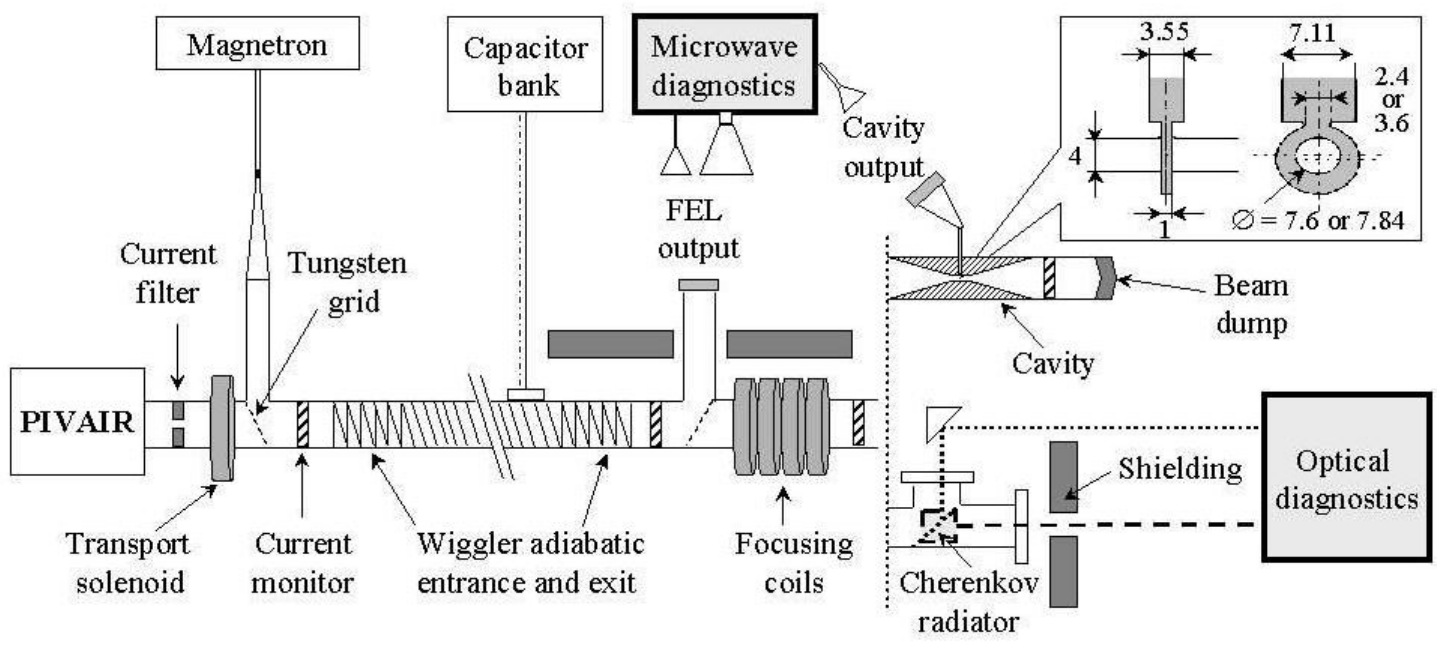

Figure B.2: Beamline layout and diagnostics for the FEL, the cavities, and beam.

\begin{tabular}{|c|c|}
\hline Wiggler period & $20 \mathrm{~cm}$ \\
\hline \hline Resonant field & $1650 \mathrm{G}$ \\
\hline Total no. periods & 32 \\
\hline Total length & $6.4 \mathrm{~m}$ \\
\hline Input signal frequency & $35.04 \mathrm{GHz}$ \\
\hline Input signal power & $5 \mathrm{~kW}$ \\
\hline DC current at entry & $830 \pm 30 \mathrm{~A}$ \\
\hline
\end{tabular}

Table B.1: FEL parameters. 
nominal value. Similarly a six-period adiabatic exit is employed to extract the bunched electron beam along the axis at the end of the wiggler. The microwave power produced in the wiggler is deflected out of the beam line by a fine tungsten wire mesh, identical to that used to inject the magnetron signal into the beam tube. Microwave power and frequency measurements are performed using calibrated attenuators, Schottky diodes, horns and waveguides for both $\mathrm{Ka}$ and $\mathrm{X}$-bands (the latter for observing power at the lower resonant frequency). Upon leaving the wiggler the bunched beam is focused by a group composed of four thick coils. The aim is to obtain a centered narrow waist at the position of the cavity, and the coils may be displaced or tilted in order to achieve this.

\section{B.1.3 FEL Measurements}

The first part of this experiment was to obtain an adequate power level with the FEL. We show in Figure B.3 the currents at the wiggler entrance and exit as a function of time (the latter has been shifted by the time of flight of electrons between the two current monitors); and the FEL power signals vs time for the upper and lower resonant frequencies, $f_{+}(35 \mathrm{GHz})$ and $f_{-}(9.5 \mathrm{GHz})$. One sees that the output power at 35 $\mathrm{GHz}$ attains its peak value only during the early part of the pulse. In the earlier LELIA experiment [104], the bunching measurement showed that the low frequency signal, which was not injected, appeared to increase towards the end of the pulse. In the PIVAIR experiment, thanks to the synchronization of the signals, we see the same effect, although at a much lower power level. We conjecture that the competition between the two frequencies may be responsible for the decrease in $f_{+}$output towards the end of the pulse.

By moving a magnet along the beam line, we could determine the peak FEL power as a function of axial distance. Such measurements were performed, and are shown in Figure B.4. Also displayed are the predictions of the code SOLITUDE for the power and the bunching parameter $b$. One sees that it does provide a good description of the variation of the peak power as a function of distance. The bunching parameter $b$ calculated by the code is $0.4 \pm 0.05$ at the end of the wiggler. In addition, the important loss of current shown in Figure B.3 is reproduced by the simulation.

\section{B.1.4 Beam Waist Location Determination}

Once suitable operation of the FEL was achieved, the optical detection line with a thin mylar target placed at the beam waist was employed to optimize the beam position and transverse size. At the waist, its diameter was about $8 \mathrm{~mm}$, as may be seen from 


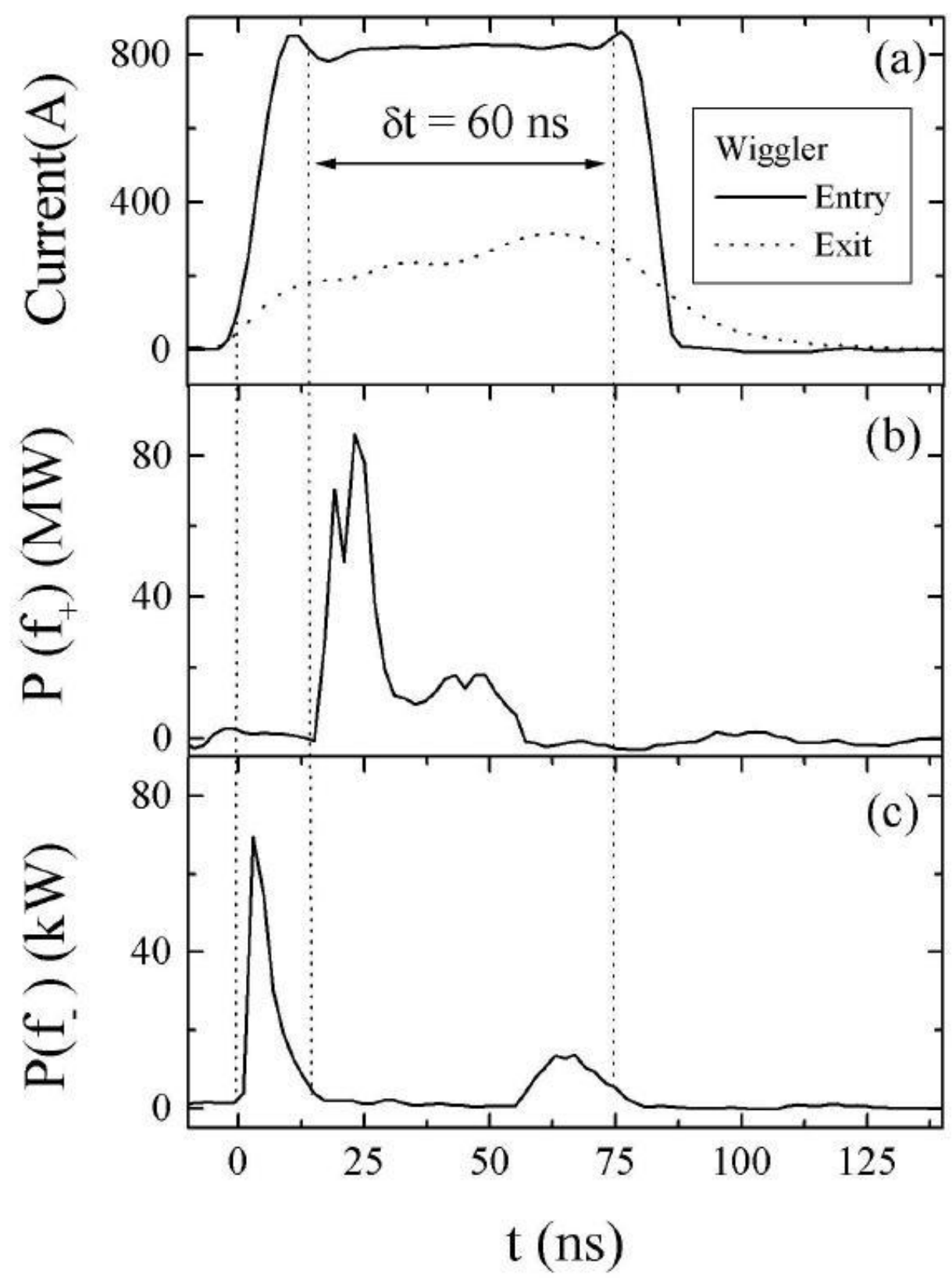

Figure B.3: Temporal evolution of beam current and output power in the FEL. 
(a)

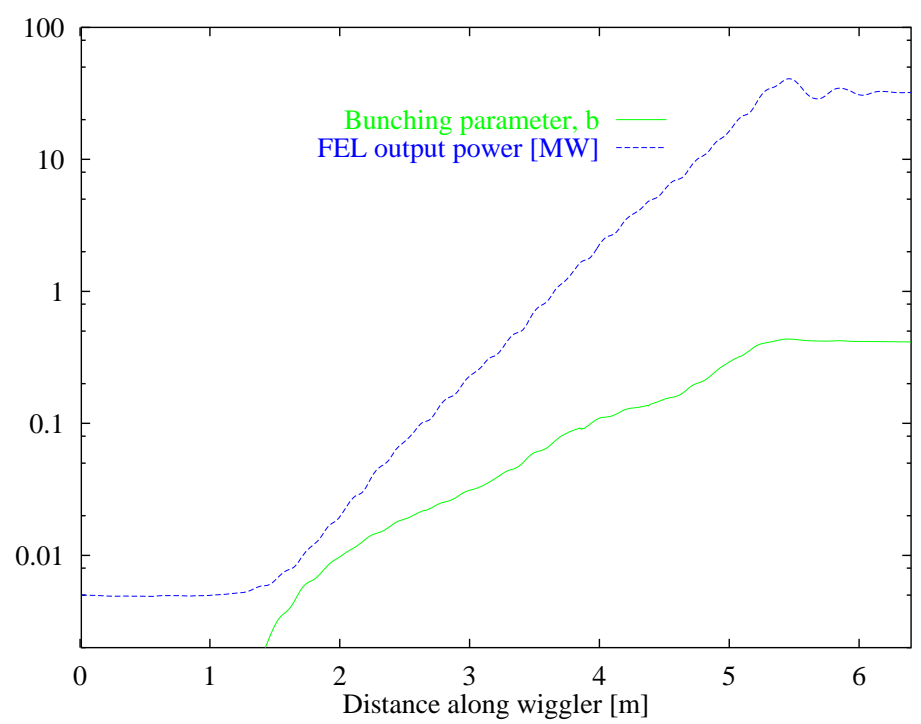

(b)

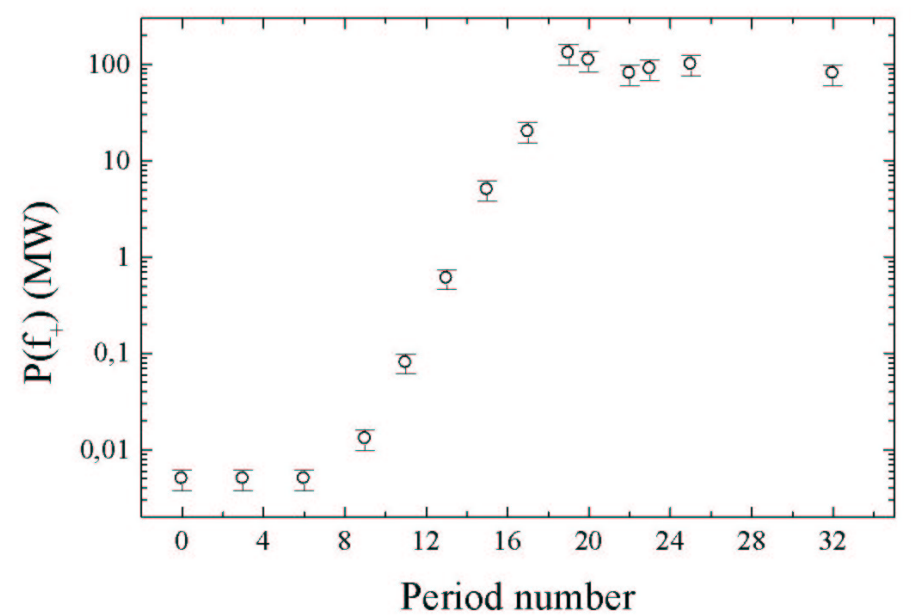

Figure B.4: (a) Evolution of FEL power and bunching in the SOLITUDE simulation; (b) Experimentally measured values. 

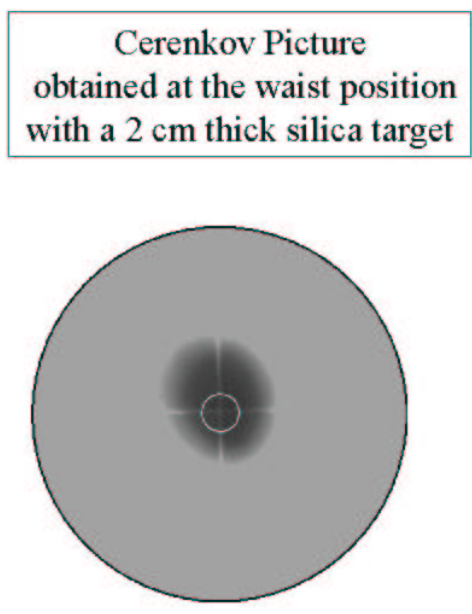

Beam radius : $\mathrm{r}_{\mathrm{RMS}}=8.1+/-0.3 \mathrm{~mm}$ Beam Offset : $x=-1 \mathrm{~mm} ; y=2.4 \mathrm{~mm}$

\section{Cavity input current}

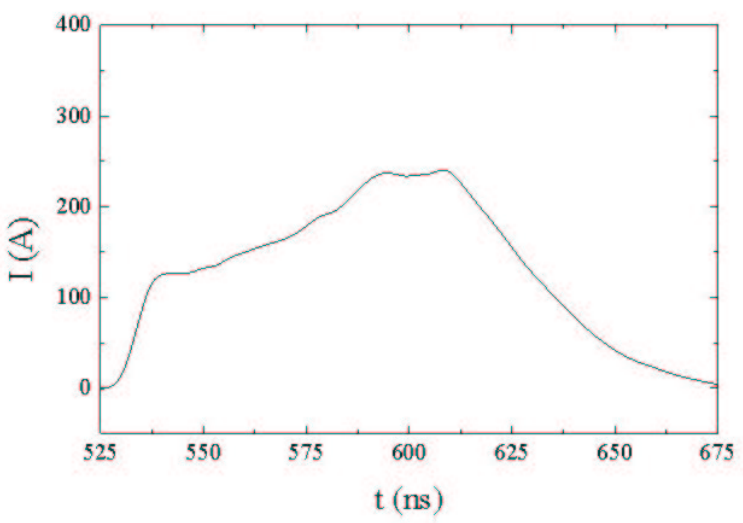

Figure B.5: Input current waveform and distribution.

the gated camera photograph displayed in Fig. B.5. Here the target is inclined at 45 degrees with respect to the beam, and photographed at 90 degrees, so that the image of the beam tube is a circle. The small circle indicates the diameter $(4 \mathrm{~mm})$ of the entrance to the cavity. Although the beam was not well-centered in this photograph, it appeared to have a roughly Gaussian profile, indicating that much of the current could be sent through the cavity if the beam were centered. At this point in course of the experiment, breakdown problems occurred in the injector, which required that the beam energy, initially $6.9 \mathrm{MeV}$, be reduced to $6.7 \mathrm{MeV}$. At this slightly different energy, higher peak FEL power (150 MW) was obtained. All subsequent measurements were made at this energy.

\section{B.1.5 Description of Cavities}

In this experiment, we used two resonant cavities (one at a time), designed at LBNL [105] and built by the CLIC group at CERN. Figure B.6shows the cavity in between the conical entrance and exit for the beam alongside details of the cavity geometry. These cavities, which operate in the TM010 mode, differ in both their resonant frequencies and Q-values. Since wall and beam-tube losses are negligible, the $Q$ values depend essentially on the coupling to the output WR28 waveguide. The low-Q cavity, with resonant frequency $f_{0}$ 


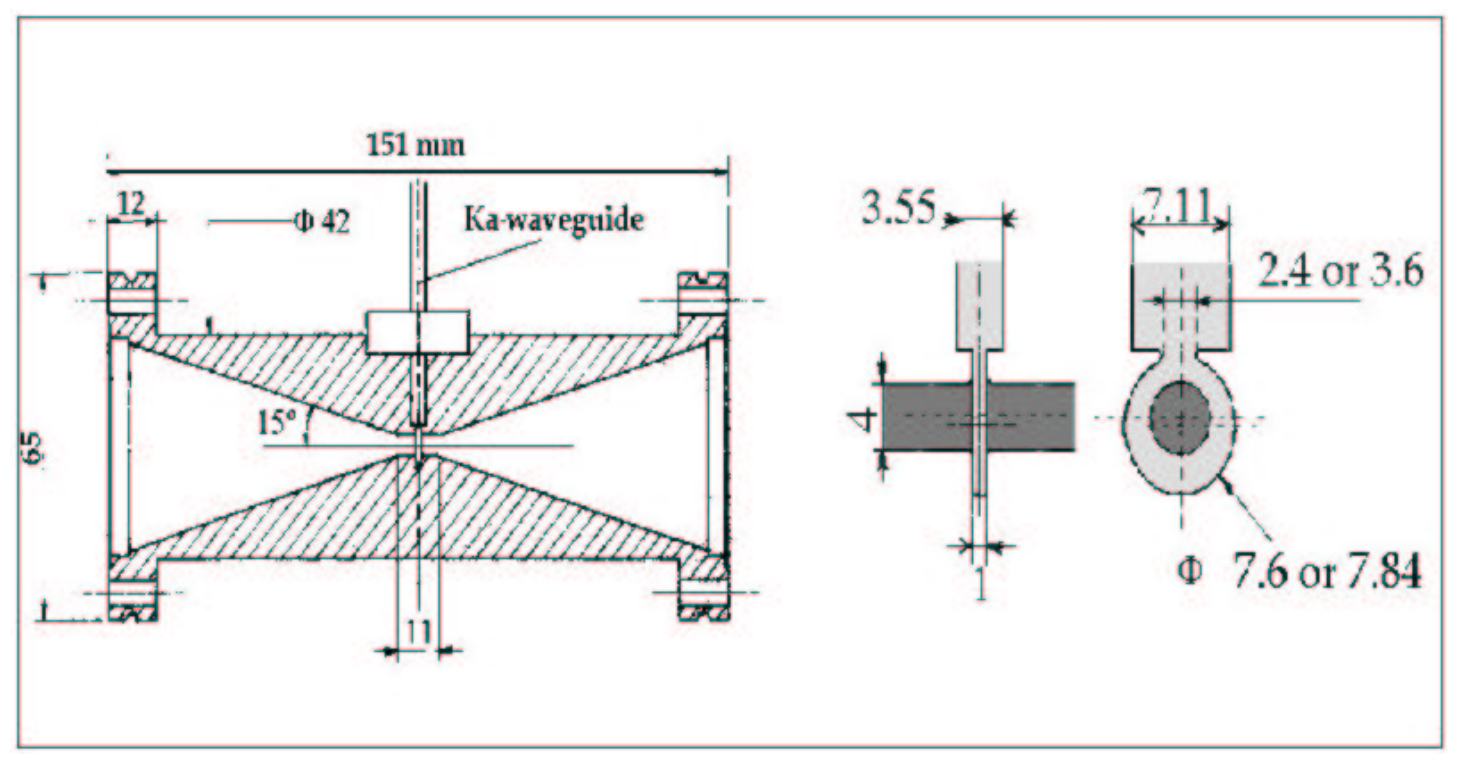

Figure B.6: Details of the standing wave rf output structures.

tuned closer to the beam modulation $\left(\mathrm{Q}=60, f_{0}=35.18 \pm 0.05 \mathrm{GHz}\right)$, extracts more power than the high-Q cavity. The latter, with its resonance inductively detuned from the modulation frequency $\left(\mathrm{Q}=270, f_{0}=35.64 \pm 0.05 \mathrm{GHz}\right)$, extracts less power, but induces a head-tail energy correlation over the bunch. This counteracts the effects of debunching due to self-field forces and incoherent energy spread, and leads to stability in the longitudinal dynamics by introducing synchrotron rotations. This is an important dynamics issue in low-energy TBA schemes and requires detailed study. In Figure B.6 we note that the axis of the beam tube and center of the cavity do not coincide, but are offset by 0.4 and $0.2 \mathrm{~mm}$, respectively, for the low-Q and high-Q cavities. This eccentricity is introduced to re-symmetrize the fields in the center of the cavity region, near the beam. This is necessary to prevent the beam from receiving an unwanted transverse kick upon traversing the cavity, and would be essential if one were passing the beam through several cavities in succession. In Figure B.7 we show contour lines of the longitudinal electric field in a cross section of the cavity, the coupling slot and the output waveguide. A cut-away view of the cavity geometry is shown in Figure B.8.We note that the beam, which had been propagating in a tube of $38 \mathrm{~mm}$ diameter, must pass through an orifice $4 \mathrm{~mm}$ in diameter. This dimension is dictated by the need to be below cut-off for the $35 \mathrm{GHz}$ radiation produced in the cavity. 


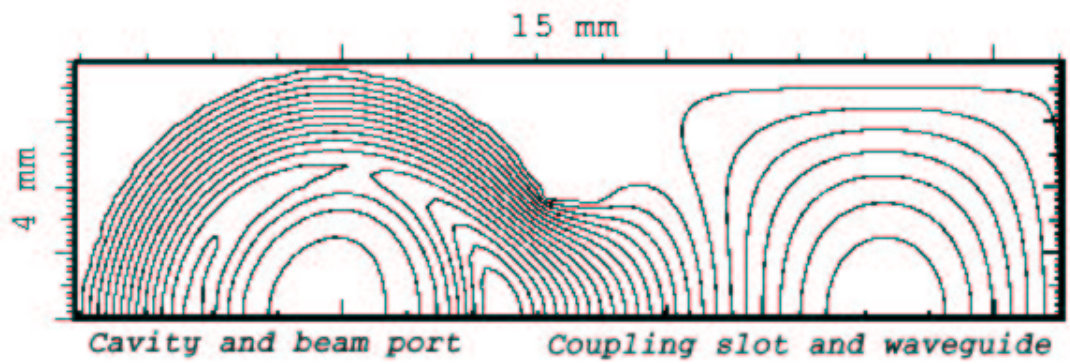

Figure B.7: Contour lines of constant longitudinal electric field.

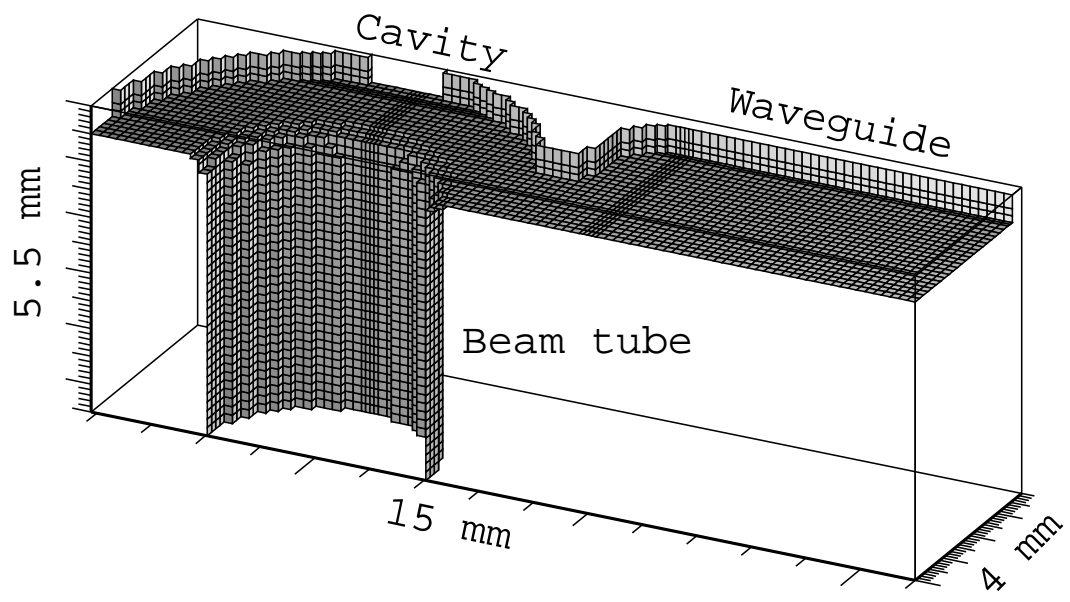

Figure B.8: Cut-away view of the cavity geometry. 


\begin{tabular}{|c|c|c|c|c|}
\hline & Low-Q $(\mathrm{Q}=60)$ & & High-Q $(\mathrm{Q}=270)$ & \\
\hline \hline Mode Frequency [GHz] & $35.18 \pm 0.05$ & & $35.64 \pm 0.05$ & \\
\hline Wiggler magnetic field [G] & $1680 \pm 40$ & $1580 \pm 40$ & $1680 \pm 40$ & $1580 \pm 40$ \\
\hline FEL Power [MW] & $150 \pm 30$ & $110 \pm 20$ & $150 \pm 20$ & $110 \pm 40$ \\
\hline Cavity Input Current [A] & $210 \pm 10$ & $210 \pm 20$ & $190 \pm 30$ & $210 \pm 10$ \\
\hline Cavity Output Current [A] & $25 \pm 5$ & $100 \pm 30$ & $50 \pm 15$ & $110 \pm 20$ \\
\hline Cavity RF Power [MW] & $0.28 \pm 0.04$ & $10 \pm 3$ & $0.06 \pm 0.03$ & $0.6 \pm 0.4$ \\
\hline Reproducibility [\# of shots] & 15 & 15 & 10 & 3 \\
\hline
\end{tabular}

Table B.2: Summary of Results

\section{B.1.6 Cavity Measurements}

Once a suitable beam waist had been obtained, the cavity was mounted, and measurements were made of power levels in both the FEL and cavity detection lines. Current monitors were placed before and after the cavity. The frequencies of both the FEL $\left(f_{+}\right)$ and the cavity radiation were found to be the same as that of the input magnetron. The main results are displayed in Table B.2. The errors shown are statistical only, based on the number of shots indicated in the last row. We observed some energy variation in the performance of the accelerator, which led us to reject a certain number of shots, limiting thereby our statistics. We note that the current measured at the entrance to the cavities is of order $200 \mathrm{~A}$. Since the beam spot size at the cavity position exceeded the aperture of the cavities (as shown in Figure B.5), only half the entrance current, at best, exited the cavity. Figure B.9 shows the temporal distribution of the current exiting the cavity, and the transverse distribution obtained from a Cerenkov foil measurement. We found that a modest $(5 \%)$ decrease in the wiggler magnetic field led to much better beam transmission through the cavity, without severely reducing the FEL output power. Thus Table B.2 has four columns, two for each cavity, corresponding to the different operating fields, 1680 and 1580 Gauss, respectively.

In Figure B.10 are shown the cavity output current, FEL output power and cavity output power for two shots, one with the low-Q $(\mathrm{a}, \mathrm{b})$ and the other with the high-Q $(\mathrm{c}, \mathrm{d})$ cavity. Again the time of flight differences have been removed to align these different curves to a common origin. Both the FEL and cavity signals are significantly shorter than the current pulse, but the peaks both coincide with the maximum of the output current. 
Cerenkov Picture taken

$12.5 \mathrm{~cm}$ long after the cavity

with a $2 \mathrm{~cm}$ thick silica target

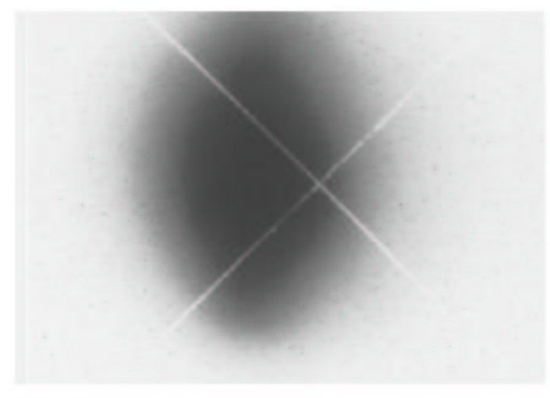

Beam radius : $\mathrm{r}_{\mathrm{RMS}}=11.5+/-1 \mathrm{~mm}$

Beam Offset: $x=-7 \mathrm{~mm} ; y=0.5 \mathrm{~mm}$

\section{Cavity ouput current}

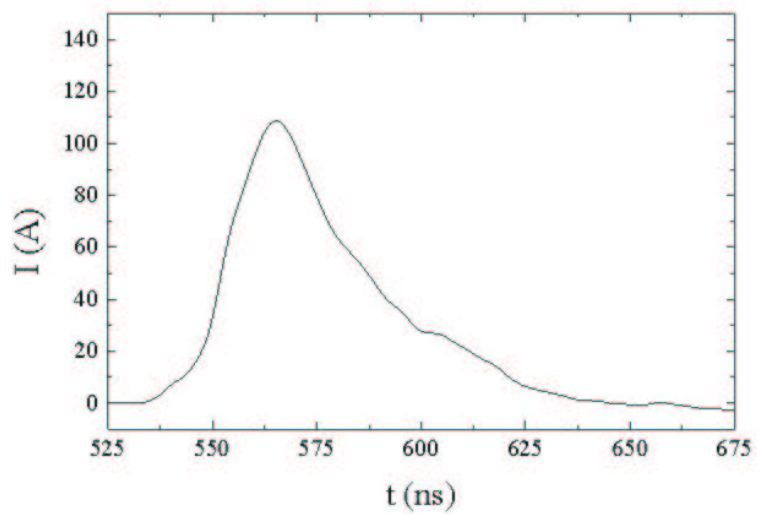

Figure B.9: Output current waveform and distribution.

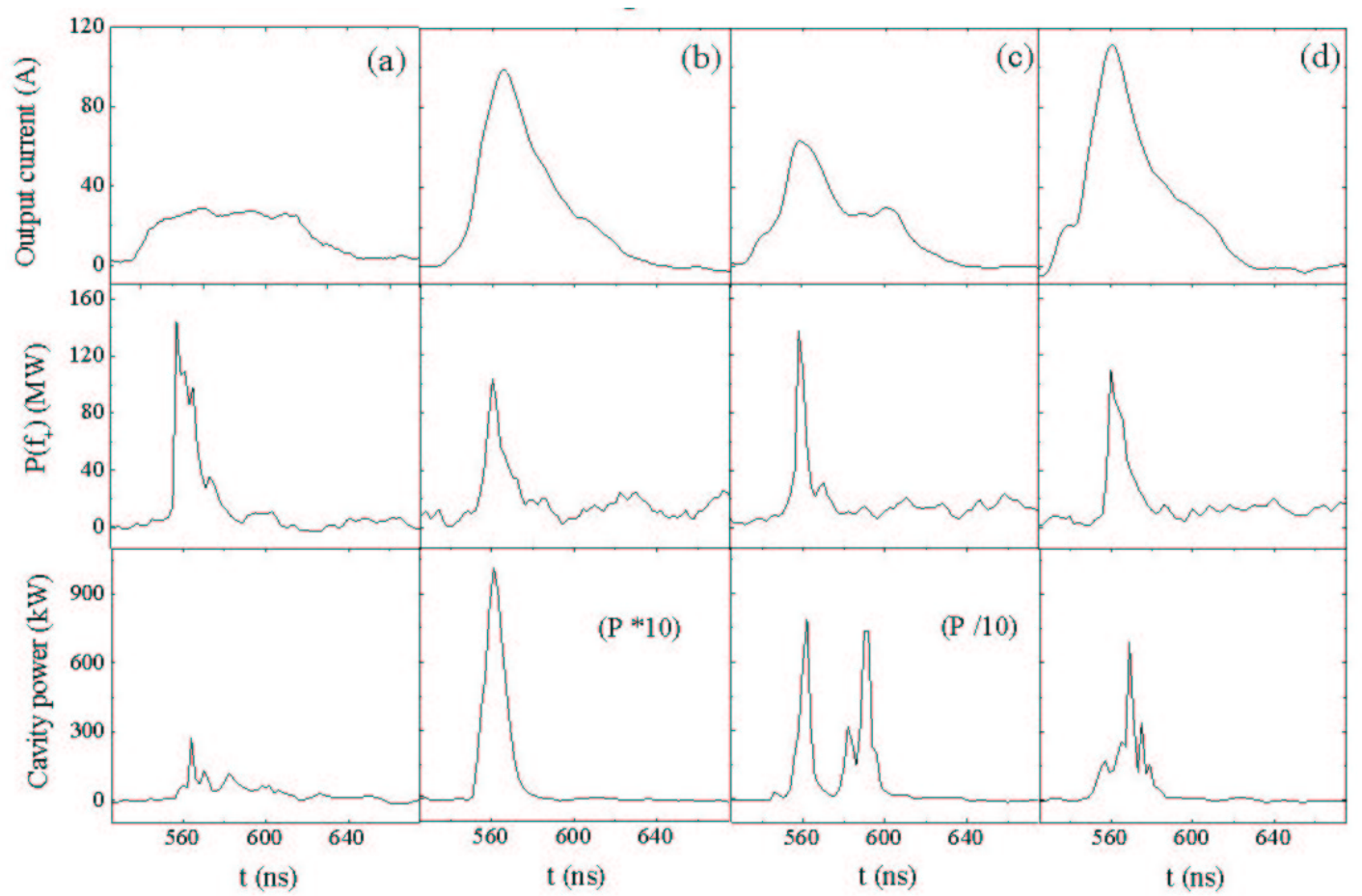

Figure B.10: Correlation between FEL, current, and cavity microwave output. 


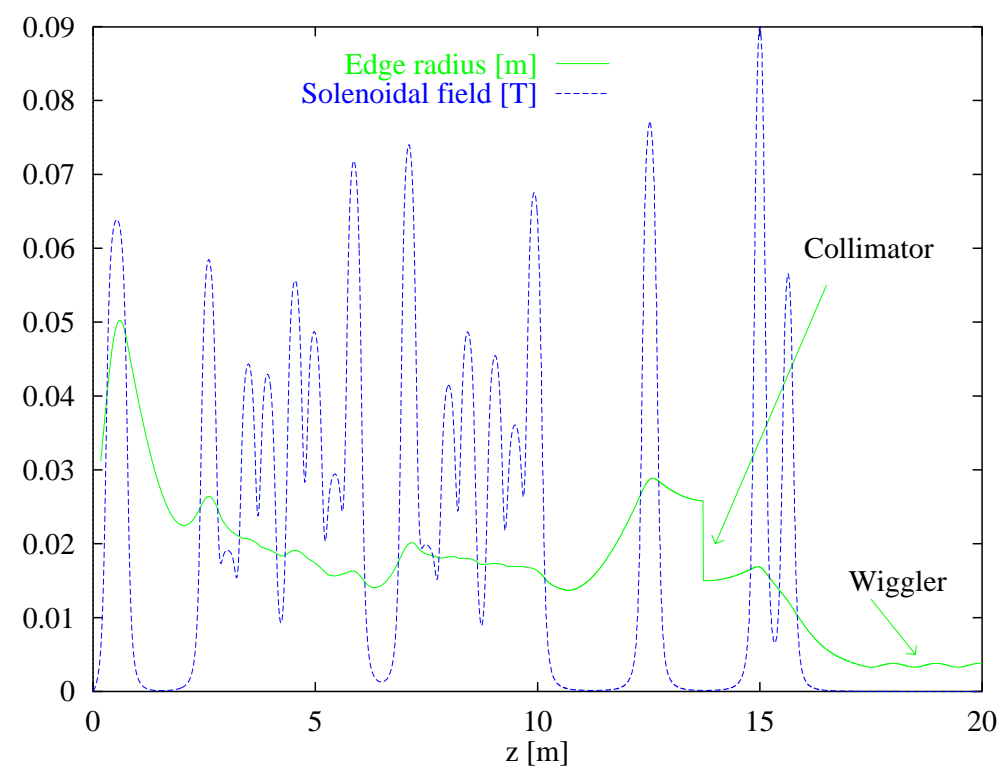

Figure B.11: Transport in PIVAIR.

\section{B.2 Simulations}

\section{B.2.1 Simulation of Transport in PIVAIR}

The simulation begins with a description of the transport from the cathode to the experiment. For this the envelope code is solely used. A plot of the magnetic field and beam edge radius is given in Figure B.11.

The currents in the two final solenoids and dipole coils are adjusted for proper matching into the wiggler. A Rogowski coil placed at the entrance to the wiggler measured the beam current; $800 \mathrm{~A}$ were obtained in the current experiment.

\section{B.2.2 Simulation of Transport through the FEL}

The main FEL parameters are listed in Table B.1. SOLITUDE has been run to obtain fair agreement with the preliminary results of the current experiment, ie. $40 \mathrm{MW}$ of FEL power and $250 \mathrm{~A}$ of current at the wiggler exit. Besides the basic parameters of the beam distribution (current, radius, emittance) and the input microwave power, SOLITUDE has a few additional 'knobs' with which to tune the resultant interaction. These allow the simulation to place the entry of the beam centroid at any radius and polar angle in the entry plane, and with two independent angles with respect to the normal of the entry plane. Sensitivity studies have shown that for small variations from 


\begin{tabular}{|c|c|c|}
\hline Resonant frequency $[\mathrm{GHz}]$ & 35.0 & 35.6 \\
\hline \hline Quality factor, $Q_{\text {Loaded }}$ & 60 & 271 \\
\hline Shunt impedance [R/Q] $[\Omega]$ & 45. & 45. \\
\hline DC current in cavity $[\mathrm{A}]$ & 120 & 120 \\
\hline Measured output power [MW] & 12 & 0.69 \\
\hline
\end{tabular}

Table B.3: RF cavity parameters.

normal, on-axis injection, it is the azimuthal angle (angular divergence from the wiggler axis) of the centroid motion which has the largest effect on overall FEL performance. In particular, we have found that an azimuthal angle of $\sim 33 \mathrm{mrad}$ at the entrance plane generates trajectories within the FEL region that lead to the observed $40 \mathrm{MW}$ of output power and 250 Amps of current at the exit current monitor. The bunching parameter at the FEL exit is given by SOLITUDE as b 0.4. The evolution of the output power and of the bunching parameter as calculated by SOLITUDE is shown in Figure B.4. The output data generated by SOLITUDE include the detailed particle distribution in the horizontal and vertical phase spaces, as well as the distribution in particle energy and arrival time for a given bunch. These data provide an indication of the initial conditions for simulations of the downstream region.

\section{B.2.3 Simulation of Downstream Transport and Cavity Interaction}

After a short drift space, the bunched beam is focused by a solenoid module (peak axial field $\sim 0.27 \mathrm{~T}$ ) into one of two Ka-band standing-wave RF output cavities. This is shown in Figure B.2. These cavities were designed using standard electromagnetic codes, and were built by the CLIC group at CERN. The measured parameters of these cavities are shown in Table B.3.

The transport from the end of the wiggler through the cavities has been studied with PARMELA, the envelope code, and RKS. The initial conditions generated by SOLITUDE have been used in the envelope simulation. In the case of the RKS code, on the other hand, we have slightly adjusted the initial conditions to obtain fair agreement with the experimentally measured beam spot sizes, and the incident and transmitted beam currents through the cavities. The trajectories calculated by the envelope code and RKS are shown in Figure B.12.

The differences between the two codes are an indication of the effects of the detailed statistics of the transverse dynamics. The envelope code assumes an on-axis beam described by a transverse KV and a longitudinally uniform distribution, while the RKS 


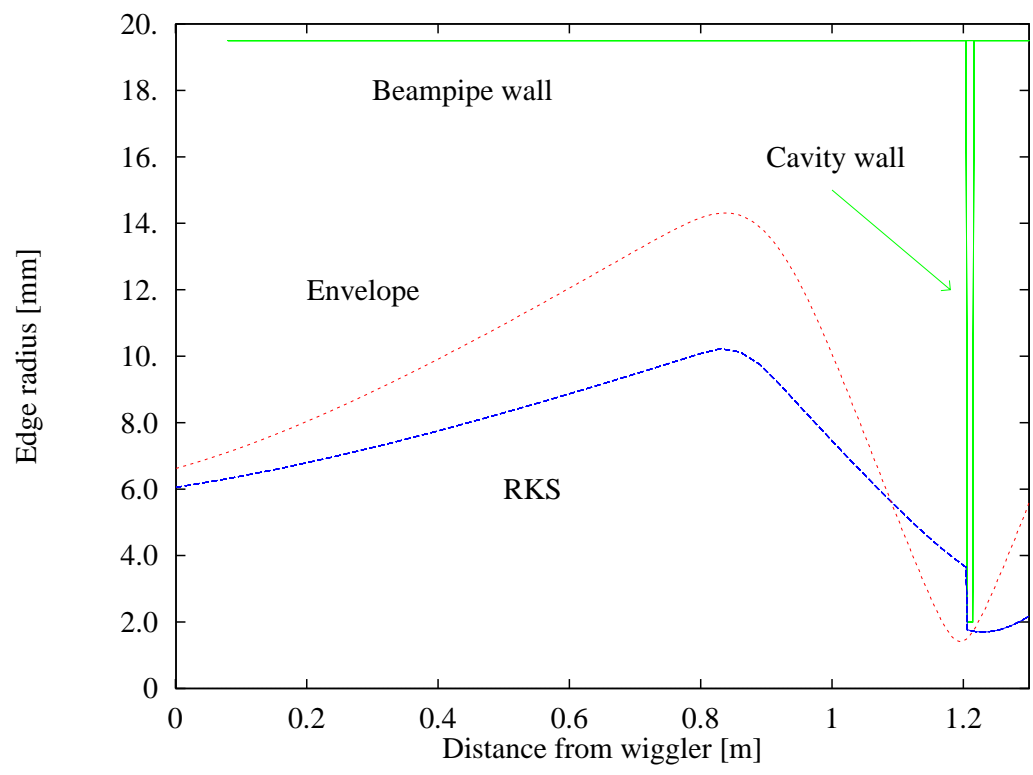

Figure B.12: Beam edge radius evolution.

code uses a semi-Gaussian distribution for the transverse phase space and a Gaussian distribution for the longitudinal phase space. The 'edge' radius in this case is twice the rms radius. These different models present slightly different modes of evolution, while showing fair agreement on the measured parameters at the end points.

Measurements of the FEL output power give an indication of the bunching parameter of the beam at the FEL exit. This has also been measured experimentally [103]. The steady-state output power from the cavities follows from the relation (equation 11.7)

$$
P_{\text {out }}=I_{d c}^{2} b^{2} Q_{\text {Loaded }}\left[\frac{R}{Q}\right] \cos ^{2} \psi
$$

where $b$ is the bunching parameter, and $\psi$ is the cavity tuning angle. The temporal evolution of the output cavity power is calculated by RKS. The output from the lowand high-Q cavities is shown in Figure B.13. The temporal evolution of the power and phase in the output cavities is calculated by an equivalent circuit equation coupled to the detailed beam dynamics [106] and Chapter 9 . 

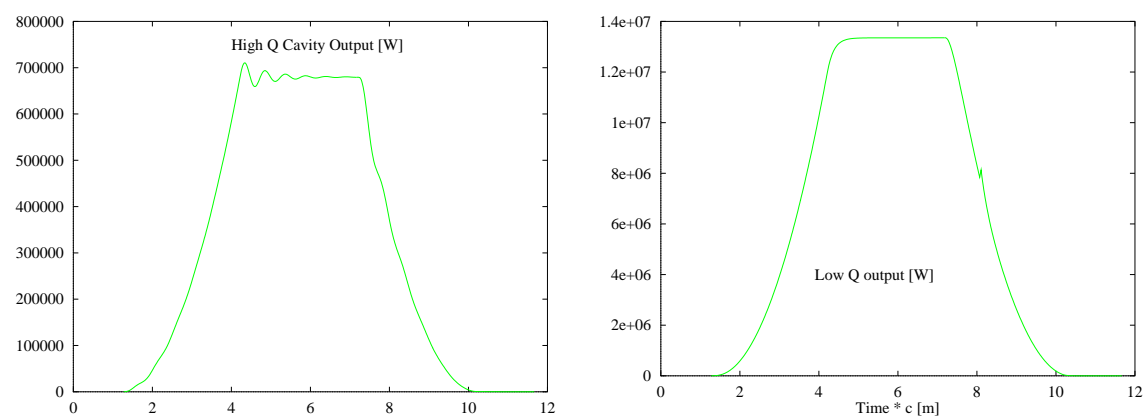

Figure B.13: Temporal power development in the two cavities.

\section{B.3 Discussion}

With a current of $200 \mathrm{~A}$, the light output from the mylar target was not sufficient to allow streak camera photography. Instead, a thick $(2 \mathrm{~cm})$ fused silica target was used as a radiator, with Cerenkov radiation in the forward direction as the source of light. Since this requires the electrons to stop in the target, straggling is appreciable and background emission is significant. Thus only a noisy optical signal was observed, which indicated that there was some bunching at $35 \mathrm{GHz}$, but did not permit a quantitative measurement. Calculations using GEANT [107] suggest that the FWHM of the light signal from a delta-function electron pulse striking the target is approximately 12 ps. This would make the observed bunching at $35 \mathrm{GHz}$ only $50 \%$ of the true value. We conclude that this method doesn't lead to a valid estimation of the bunching parameter without further experimental investigation.

In the absence of an adequate measurement of b, we must rely on a theoretical estimate. To obtain this, we use the results of the code SOLITUDE for the electron distribution in six-dimensional phase-space at the exit of the wiggler. This is used as input into RKS, which then calculates the evolution of the phase-space density during the transport to and through the cavity. This calculation supplies us with the current, bunching and emittance of the beam as a function of axial distance. As we noted previously, SOLITUDE predicted $b=0.4 \pm 0.05$ at the wiggler exit. The prediction of RKS is that the bunching parameter increases throughout the transport and focusing region, reaching a value of 0.5 , while the current decreases from $250 \mathrm{~A}$ at the wiggler exit to $200 \mathrm{~A}$ at the entrance to the cavity. When the beam enters the cavity, a further decrease in the current to $120 \mathrm{~A}$ is accompanied by a sharp increase of the bunching parameter to 0.6 .

Although we don't have an experimental measure of b, we may nonetheless evalu- 
ate the power formula using the theoretical estimate provided by the codes. We find, assuming $\mathrm{I}=100 \mathrm{amps}, \mathrm{b}=0.6$, and $\left[\frac{R}{Q}\right]=45 \Omega$, output power of $8 \mathrm{MW}$ for the low-Q and $520 \mathrm{~kW}$ for the high-Q cavity. These numbers are comparable to the peak power levels shown in Figure B.10, and we are confident that b is at least 0.5 in the cavity. In addition, the large variation in output power seen for our two choices of wiggler field can be explained by the large difference in current transmission and a modest variation in the bunching parameter. 


\section{Bibliography}

[1] A.M. Sessler. The free electron laser as a power source for a high-gradient accelerating structure. In P.J. Channell, editor, Laser Acceleration of Particles, number 91 in AIP Conference Proceedings, pages 154-9, 1982.

[2] A. Sessler and S.S. Yu. Relativistic klystron two-beam accelerator. Phys. Rev. Lett., 54:889, 1985.

[3] H. Murayama and M.E. Peskin. Physics opportunities of e $+\mathrm{e}-$ linear colliders. In Ann. Rev. Nucl. Part. Sci., volume 46, pages 533-608. Annual Reviews Incorporated, 1996.

[4] The NLC ZDR Design Group and The NLC Physics Working Group. Physics and technology of the next linear collider. Technical Report BNL 52-502, FermilabPUB-96/112, LBNL-PUB-5425, SLAC Report 485, UCRL-ID-124160, BNL, Fermilab, LBNL, SLAC, June 1996.

[5] M. Carena et al. Searches for supersymmetric particles at the tevatron collider. Rev. Mod. Phys., 71(4):937-981, July 1999.

[6] J. Seeman. The stanford linear collider. In Ann. Rev. Nucl. Part. Sci., volume 41, pages 389-428. Annual Review, Inc., Palo Alto, 1991.

[7] K. Yokoya and P. Chen. Beam-beam phenomena in linear colliders. In Frontiers of Particle Beams: Intensity Limitations, volume 400 of Lecture Notes in Physics, pages 415-45. Springer-Verlag, 1992.

[8] R.B. Palmer. Prospects for high energy e+e- linear colliders. Ann. Rev. Nucl. Part. Sci., 40:529-92, 1990. 
[9] P.B. Wilson. Application of high-power microwave sources to tev linear colliders. In A.V. Gaponov-Grekhov and V.L. Granatstein, editors, Application of High-Power Microwaves. Artech House, 1994.

[10] J. Irwin. Bird's ip view of limits of conventional e+e- linear collider technology. In Proceedings of the 6th Workshop on Advanced Accelerator Concepts, Lake Geneva, Wisconsin, 12-18 June, 1994, 1994.

[11] T.O. Raubenheimer. Tolerances to limit the vertical emittances in future storage rings. Part. Acc., 36:75-119, 1991.

[12] O. Napoly. Tesla general design overview. In Proceedings of the VII International Workshop on Linear Colliders, Zvenigorod, Russia, 1997.

[13] The NLC Design Group. Zeroth-order design report for the next linear collider. Technical Report LBNL-PUB-5424, SLAC Report 474, UCRL-ID-124161, SLAC, LBNL, LLNL, and others, 1996.

[14] The CLIC Study Team. Clic, a 0.5 to 5 tev e+e- compact linear collider. In Proceedings of the 6th European Particle Accelerator Conference, 1998.

[15] G.A. Loew and T. Weiland (eds.). International linear collider technical review committee ilc-trc report 1995. Technical report, SLAC, 1995.

[16] E.L. Ginzton et al. A linear electron accelerator. Rev. Sci. Instrum., 19:89-108, 1948.

[17] M. Chodorow et al. Stanford high-energy linear electron accelerator (mark iii). Rev. Sci. Instrum., 26:134-204, 1955.

[18] J.C. Slater. Microwave Electronics. D. Van Nostrand Company, Inc., 1950.

[19] D.H. Whittum. Introduction to electrodynamics for microwave linear accelerators. In RF Engineering for Particle Accelerators. World Scientific, 1998.

[20] T. Wangler. RF Linear Accelerators. John Wiley and Sons, Inc., 1998.

[21] V.K. Neil and R.K. Cooper. Coherent instabilities in high current linear induction accelerators. Particle Accelerators, 1:111-120, 1970.

[22] R.L. Gluckstern et al. Cumulative beam breakup in rf linacs. Particle Accelerators, $16: 125-153,1985$. 
[23] S.M. Lidia et al. Relativistic klystron two-beam accelerator approach to multi-tev e+e- linear colliders. In New Directions for High-Energy Physics, Proceedings of the 1996 DPF/DPB Summer Study on High-Energy Physics, Snowmass, Colorado, 1996.

[24] R.H. Fowler and L. Nordheim. Field emission. Proc. Roy. Soc., A119:173, 1928.

[25] G.A. Loew and J.W. Wang. Rf breakdown, field emission and dark current. In A.W Chao and M. Tigner, editors, Handbook of Accelerator Physics and Engineering, chapter 6.12, pages 390-2. World Scientific, 1998.

[26] G.A. Loew and J.W. Wang. Rf breakdown studies in room temperature electron linac structures. Technical Report SLAC-PUB-4647, SLAC, May 1988.

[27] A. Septier and P.M. Lapostolle eds. Linear Accelerators. North-Holland Pub., Amsterdam, 1970.

[28] R. Assmann et al. Observation of dark-current signals from the s-band structures of the slac linac. Technical report, Stanford Linear Accelerator Center, July 1997.

[29] W.D. Kilpatrick. Criterion for vacuum sparking designed to include both rf and dc. Rev. Sci. Instrum., 28(10):824-6, 1957.

[30] J.W. Wang et al. Slac-pub-5900. Technical report, Stanford Linear Accelerator Center, 1992.

[31] O.A. Nezhevenko. On the limitations of accelerating gradient in linear colliders due to the pulse heating. In Proceedings of the 1997 Particle Accelerator Conference, Vancouver, Canada, 1997.

[32] D.P. Pritzkau. Possible high power limitations from rf pulsed heating. Technical report, Stanford Linear Accelerator Center, November 1998.

[33] P.B. Wilson. Scaling linear colliders to 5 tev and above. In Presented at the ITP Conference on Future Linear Colliders, University of California, Santa Barbara, October 1996.

[34] J. Benford and J. Swegle. High-Power Microwaves. Artech House, 1992.

[35] C.A. Kapetanakos and P. Sprangle. Ultra-high current electron induction accelerators. Physics Today, 38:58, 1985. 
[36] R.J. Adler and J.R. Bayless. Linear induction accelerators for industrial applications. In Proceedings of the IEEE Particle Accelerator Conference, Washington, D.C., volume 3, 1987.

[37] T.J. Orzechowski et al. Microwave radiation from a high-gain free-electron laser amplifier. Phys. Rev. Lett., 54:889, 1985.

[38] T.J. Orzechowski et al. High efficiency extraction of microwave radiation from a tapered wiggler free electron laser. Phys. Rev. Lett., 57:2172, 1986.

[39] M.A. Allen et al. Relativistic klystron research for high gradient accelerators. Technical report, Stanford Linear Accelerator Center and Lawrence Livermore National Laboratory, June 1988.

[40] M.A. Allen et al. High gradient electron accelerator powered by a relativistic klystron. Phys. Rev. Lett., 63:2472, 1989.

[41] T.L. Houck. Study of a Microwave Power Source For A Two-Beam Accelerator. PhD thesis, University of California, Davis, 1994.

[42] G.A. Westenskow and T.L Houck. Results of the reacceleration experiment: Experimental study of the relativistic-klystron two-beam accelerator concept. In Proceedings of the 10th International Conference on High Power Particle Beams, San Diego, CA, June 1994.

[43] S. Lidia et al. Rk-tba studies at the rta test facility. In Proceedings of the 7 th Advanced Accelerator Concepts Workshop, Lake Tahoe, California, 1996.

[44] J. Barnard et al. Study of recirculating induction accelerators as drivers for heavy ion fusion. Technical Report UCRL-LR-108095, LLNL, 1991.

[45] K. Halbach. unpublished.

[46] K. Halbach. Design of permanent multipole magnets with oriented rare earth cobalt material. Nucl. Instrum. Meth. Phys. Res., 169:1-10, 1980.

[47] Y-J Chen. Corkscrew modes in linear accelerators. Nucl. Instrum. Meth. Phys. Res., A292:455-464, 1990.

[48] J. Clark et al. Design and initial operation of the eta-ii induction accelerator. In Proceedings of the XIV International Linear Accelerator Conference, Williamsburg, $V A$, pages 19-23, 1988. 
[49] T.L. Houck et al. Stacked insulator induction accelerator gaps. In Proceedings of the IEEE Particle Accelerator Conference, Vancouver, Canada, 1997.

[50] T.L. Houck et al. Measured and theoretical characterization of the rf properties of stacked, high-gradient insulator material. In Proceedings of the IEEE Particle Accelerator Conference, Vancouver, Canada, 1997.

[51] J. Haimson and B. Mecklenburg. Design and construction of a chopper driven 11.4 ghz travelling wave rf generator. In Proceedings of the IEEE Particle Accelerator Conference, pages 243-245, 1989.

[52] S. Gold and G.S. Nusinovich. Review of high-power microwave source research. Rev. Sci. Instrum., 68:3945, 1997.

[53] G.A. Westenskow and T.L. Houck. Relativistic klystron two-beam accelerator. IEEE Trans. Plasma Sci., 22:750-755, 1994.

[54] E. Sternbach. A steady-state fel: particle dynamicsin the fel portion of a two-beam accelerator. Nucl. Instrum. Methods Phys. Res., A250:464-75, 1986.

[55] N.M. Kroll et. al. Free-electron lasers with variable parameter wigglers. IEEE J. Quant. Elec., QE-17:1436-1468, 1981.

[56] R.D. Ryne and S.S. Yu. Relativistic klystron simulations using rktw2d. In Proceedings of the Linear Accelerator Conference, Albuquerque, NM, pages 177-179, 1990.

[57] G. Fiorentini. Numerical simulation of relativistic klystrons. In Proceedings of the 1993 Computational Accelerator Physics Conference (CAP93), Los Alamos, pages 367-76, 1994.

[58] T.L. Houck et al. Bbu code development for high-power microwave generators. In Proceedings of the XVI International Linear Accelerator conference, Ottowa, page $495,1992$.

[59] W.K.H. Panofsky and M. Bander. Asymptotic theory of beambreak-up in linear accelerators. Rev. Sci. Instrum., 39:206, 1968.

[60] V.I. Arnold. Mathematical Methods of Classical Mechanics. Springer-Verlag, 1989.

[61] H. Goldstein. Classical Mechanics. Addison-Wesley Publishing Company, 1980. 
[62] B. Schutz. Geometrical Methods of Mathematical Physics. Cambridge University Press, 1980.

[63] A.J. Dragt. Lie algebraic treatment of linear and nonlinear beam dynamics. In Ann. Rev. Nucl. Sci., volume 38, pages 455-496. Annual Reviews Incorporated, 1988.

[64] K.L. Brown and R.V. Servranckx. SLAC-PUB-3381, July 1984.

[65] H. Wollnik. Optics of Charged Particles. Academic Press Inc., 1987.

[66] H. Wollnik and M. Berz. Relations between elements of transfer matrices due to the condition of symplecticity. Nucl. Instrum. Methods Phys. Res., A238:127-40, 1985 .

[67] A.J. Dragt. Lectures on nonlinear orbit dynamics. In R.A. Carrigan et. al., editor, Physics of High Energy Particle Accelerators, pages 147-313. American Institute of Physics, 1982.

[68] C. Lanczos. The Variational Principles of Mechanics. University of Toronto Press, 1970.

[69] A.J. Dragt and J. Finn. Lie series and invariant functions for analytic symplectic maps. J. Math. Phys., 17(12):2215-2227, 1976.

[70] D.H. Sattinger and O.L. Weaver. Lie Groups and Algebras with Applications to Physics, Geometry, and Mechanics. Springer-Verlag, 1986.

[71] A.J. Dragt and E. Forest. Computation of nonlinear behavior of hamiltonian systems using lie algebraic methods. J. Math. Phys., 24(12), 1983.

[72] A.J. Lichtenberg and M.A. Lieberman. Regular and Chaotic Dynamics. SpringerVerlag, 2nd edition, 1992.

[73] R. Ryne and S. Habib. Parallel beam dynamics calculations on high performance computers. In J.J. Bisognano and A.A. Mondelli, editors, Computational Accelerator Physics, number 391 in AIP Conference Proceedings, pages 377-388. American Institute of Physics, 1997.

[74] R.D. Ruth. A canonical integration technique. IEEE Trans. Nucl. Sci., NS$30: 2269-2271,1983$. 
[75] E. Forest and R.D. Ruth. Fourth-order symplectic integration. Physica D, 43:105$117,1990$.

[76] H. Yoshida. Construction of higher-order symplectic integrators. Phys. Lett. A, $150: 262-268,1990$.

[77] E. Forest et. al. Application of the yoshida-ruth techniques to implicit integration and multi-map explicit integration. Physics Letters A, 158:99-101, 1991.

[78] J.D. Jackson. Classical Electrodynamics. John Wiley and Sons Inc., 2nd edition, 1975.

[79] E. Butkov. Mathematical Physics. Addison-Wesley Publishing Co., Reading, MA, 1968.

[80] H. Wiedemann. Particle Accelerator Physics. Springer-Verlag, 1993.

[81] X.M. Marechal et. al. Magnetic field generated by parallelepiped permanent magnet block. Technical Report ESRF-SR/ID-90-37, ESRF, 1990.

[82] C.K. Birdsall and A.B. Langdon. Plasma Physics Via Computer Simulation. IOP Publishing Ltd., 1991.

[83] R.W. Hockney and J.W. Eastwood. Computer Simulation Using Particles. IOP Publishing Ltd., Bristol, 1988.

[84] W. Hackbusch. Multi-Grid Methods and Applications. Springer-Verlag, Berlin, 1980.

[85] G.D. Smith. Numerical Solution of Partial Differential Equations: Finite Difference Methods. Clarendon Press, Oxford, third edition, 1985.

[86] W. Press et. al. Numerical Recipes in Fortran: The Art of Scientific Computing. Cambridge University Press, New York, NY, second edition, 1992.

[87] I.M. Kapchinskij and V.V. Vladimirskij. Limitations of proton beam current in a strong focusing linear accelerator associated with the beam space charge. In Proceedings of the 2nd International Conference on High Energy Accelerators, page 274, CERN, Geneva, 1959.

[88] D.R. Nicholson. Introduction to Plasma Physics. Krieger Publishing House, 1983. 
[89] E. Forest et. al. Correct local description for tracking in rings. Particle Accelerators, $45(2): 65-94,1994$.

[90] M.D. Feit and J.A. Fleck Jr. Light propagation in graded-index optical fibers. Applied Optics, 17(24):3990, 1978.

[91] R.D. Ryne (LANL), private communication.

[92] P.M. Lapostolle. Possible emittance increase through filamentation due to space charge in continuous beams. IEEE Trans. Nucl. Sci., NS-18:1101, 1971.

[93] F. Sacherer. Rms envelope equations with space charge. IEEE Trans. Nucl. Sci., NS-18:1105, 1971.

[94] W.K.H. Panofsky and W.A. Wenzel. Some considerations concerning the transverse deflection of charged particles in radio-frequency fields. Rev. Sci. Instrum., $27: 967,1956$.

[95] T. Houck and G.A. Westenskow. Prototype microwave source for a relativistic klystron two-beam accelerator. IEEE Trans. Plasma Sci., 24(3):938, 1996.

[96] Registered name of Corning Glass Works.

[97] Registered name of Allied Signal Corporation.

[98] C. Smith and L. Barberi. Dynamic magnetization of metallic glasses. In Proceedings of the 5th IEEE International Pulsed Power Conference, 1985.

[99] T.L. Houck et al. Diagnostics for a 1.2-ka, 1-mv electron induction injector. In Proceedings of the 8th Beam Instrumentation Workshop, SLAC, 1998.

[100] Parmela. Maintained by the Los Alamos Accelerator Code Group.

[101] J. Gardelle et al. Free-electron laser simulations: Effects of beam quality and space charge. Phys. Rev. E, 50:4973, 1994.

[102] W. Bruns. Gdfidl: A finite difference program with reduced memory and cpu usage. In Proceedings of the IEEE Particle Accelerator Conference, page 2561, Vancouver, 1997.

[103] J. Gardelle et al. Direct observation of beam bunching produced by a high power microwave free electron laser. Phys. Rev. E, 79:3905, 1997. 
[104] T. Lefevre et al. Self-amplified spontaneous emission and bunching at 3 ghz in a microwave free-electron laser. Phys. Rev. Lett., 1999.

[105] S.M. Lidia et al. Rk-tba studies in ka band. In Proceedings of the XIX International Linear Accelerator Conference, Chicago, Ill., page 97, 1998.

[106] S. Lidia. Single-mode beam-cavity interaction in relativistic klystrons. In Proceedings of the 1999 IEEE Particle Accelerator Conference, New York, NY, 1999.

[107] F. Carminati et al. The GEANT Manual. CERN, Geneva, 1996. 